\title{
Relating Neurochemical Changes Associated with THC Use to Learning and Memory in Adolescent Rats
}

by

\section{Ryan William Johnstone Steel}

A thesis submitted to the Victoria University of Wellington

in fulfilment of the requirements for the degree of

Doctor of Philosophy in Biomedical Science

Victoria University of Wellington 


\section{Abstract}

Cannabis is the most widely used illicit drug. Adolescents may be especially vulnerable to the effects of cannabis, and alarmingly, adolescence is also a period of heavy cannabis use. However, few studies have investigated the cognitive effects of cannabis use in adolescents specifically. Furthermore, the neurochemical correlates of cognitive impairment associated with cannabis use at any age have received very little experimental attention. This research project sought to address these shortcomings in the literature using THC, the major psychoactive component of cannabis, and a rat model of adolescence.

The rate of learning was slower in THC-treated animals, and this was attributable to deficits in the cognitive function of 'chunking', a process by which the information capacity of short-term memory is enlarged. Impairment of chunking by cannabinoids has not been previously reported. Behavioural impairment by THC was associated with impaired hippocampal plasticity, including changes in synaptic activity and architecture, as well as changes in neurogenesis. The attenuation of structural and functional plasticity in the hippocampus in response to training in a learning task was more pronounced than the subtle effects of THCtreatment on the survival and early development of newborn neurons. Importantly, no effects of THC were seen in animals not trained in the maze. Thus, plasticity is more sensitive to the effects of THC during times of learning, and this greater sensitivity likely accounts for the behavioural impairment associated with cannabis use.

The data presented in this thesis add significantly to the existing literature by identifying novel behavioural and neurochemical processes by which cannabis use may impair learning and memory. Whether these impairments represent a greater sensitivity of adolescents to THC remains to be determined. 


\section{Acknowledgements}

The completion of a PhD is a significant individual achievement that requires the support of many others.

My supervisors, Dr. Darren Day and Prof. John Miller, deserve significant praise and credit for the enthusiasm and patience they have shown me throughout this project. The ideas, expertise and knowledge that each of you has brought to our discussions have greatly influenced not only this research, but also my personal development as a critically thinking scientist. A special thank you to John Miller for his generous support of my conference attendance and super speedy reviewing of chapters!

A/Prof. David Harper and Prof. Susan Schenk of the School of Psychology at VUW deserve credit for their guidance in matters of behavioural testing and basic experimental design. Thank you also to Richard Moore for organising access to animals for this research.

Thank you to the technicians and academic staff of SBS, including Dr. Bronwyn Kivell, A/Prof. Anne La Flamme, Cameron Jack and Angela Fleming, as well as the administration staff, Mary Murray and Patricia Stein in particular.

Thank you to my fellow KK801 lab mates - Dr. Tim Sargeant, Dr. Anasuya Vishvanath, Darryl Jones, Andy Douglass and Dr. Tinte Itering.

Thank you to everyone who has helped to make this a bearable experience - Ben Reuhman, Bridget Simonson, Kirsty Danielson, Aashish Morani, Peter Bosch, Arun Kanakkanthara, Bede Busby, Dave O'Sullivan, Ariane Chan, Hannah Hoang, Dr. Matthew Gerrie, Dr. Lincoln Hely, Caleb Carati, and many others!

Thank you to my father Kevin, mother Judy, and sister Charlotte Steel. You were always there when I needed you most. I love you all.

Finally, my love and gratitude goes to Jessica Field. Without your understanding, love, support and attention it would have taken me several more years more to be where I am today. Thank you!

This work was supported by funding from the Wellington Medical Research Foundation, The Neurological Foundation of New Zealand, and Victoria University of Wellington. 


\section{Table of Contents}

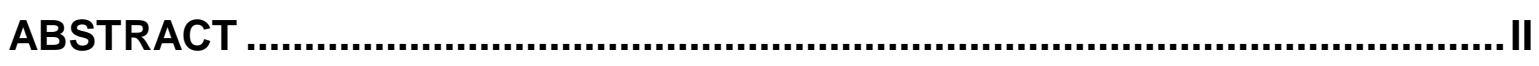

ACKNOWLEDGEMENTS...............................................................................

TABLE OF CONTENTS .............................................................................

LIST OF FIGURES ..................................................................................II

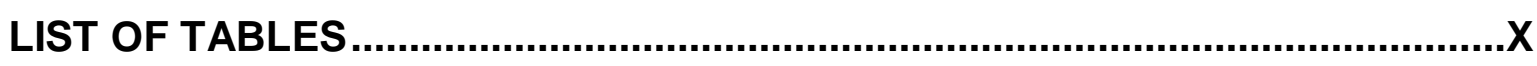

LIST OF COMMONLY USED ABBREVIATIONS ...............................................

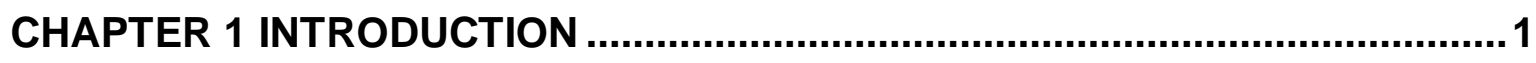

1.1 Cannabis Use: Historical Aspects and the New Zealand Perspective ........................ 1

1.2 Molecular Biology and Biochemistry of the Cannabinoid System ............................. 2

1.2.1 Molecular Biology of Cannabinoid Receptors ........................................................ 2

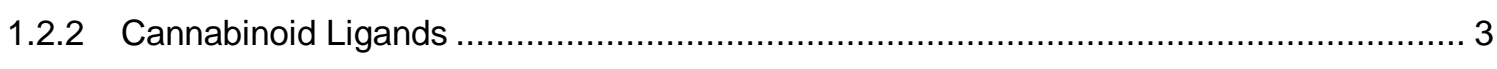

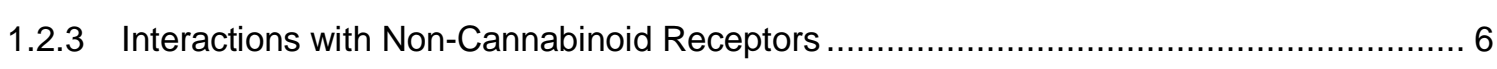

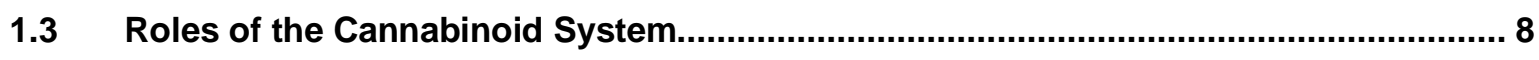

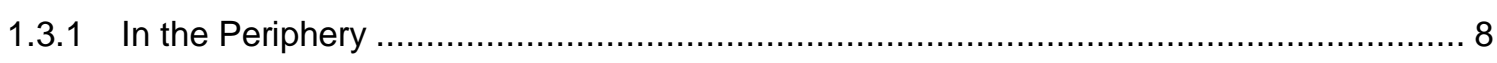

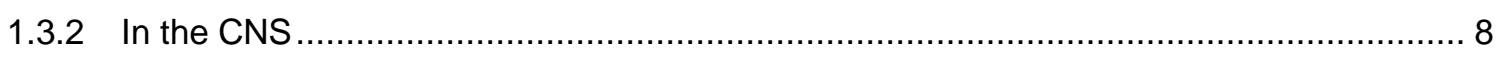

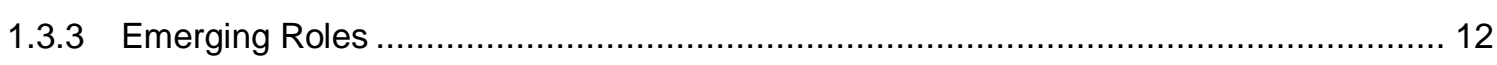

1.4 Behavioural Consequences of Cannabinoid Exposure ............................................ 15

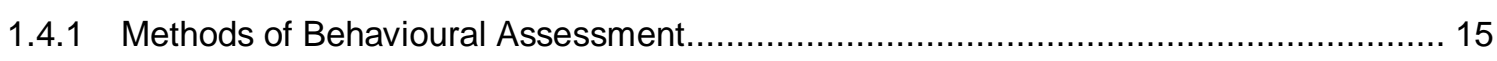

1.4.2 Consequences of Exposure in Adulthood ............................................................ 18

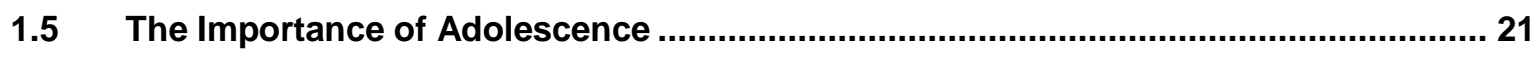

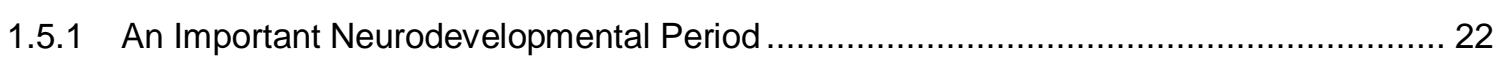

1.5.2 Behavioural Effects of Adolescent Cannabinoid Exposure ........................................ 23

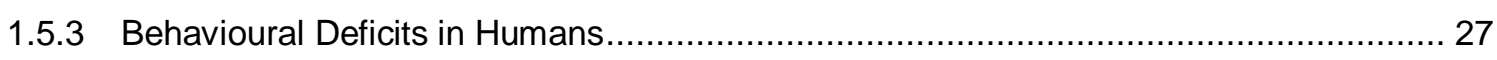

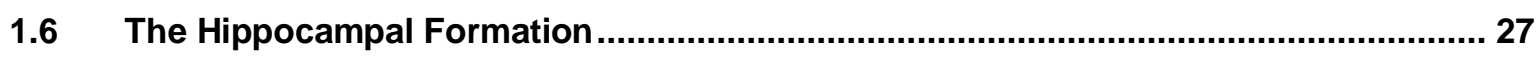

1.6.1 Subfields and Neural Pathways of the Hippocampal Formation ................................. 27

1.6.2 Importance of the Hippocampus in Learning and Memory ...................................... 30 


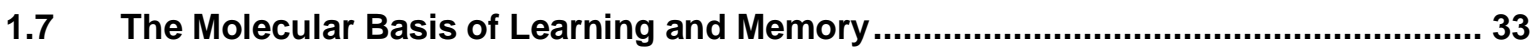

1.7.1 Senescence as a Model for Impairment in Learning and Memory Tasks ....................... 33

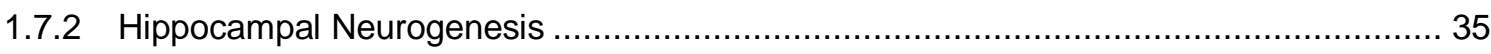

1.7.3 Presynaptic Mechanisms of LTP and Synaptogenesis .............................................. 41

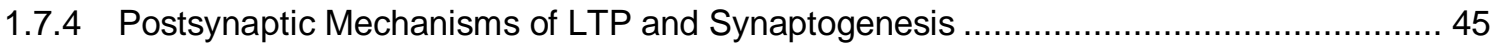

1.8 The Effect of Cannabinoids on Hippocampal Plasticity............................................ 49

1.8.1 Cannabinoid Influences on Neurogenesis ........................................................... 49

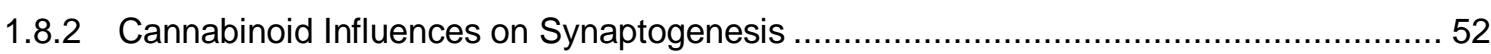

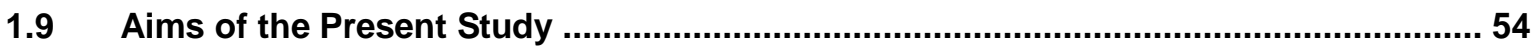

CHAPTER 2 GENERAL METHODS .......................................................... 56

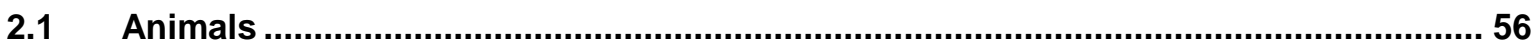

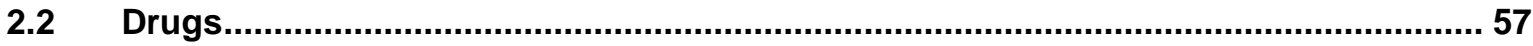

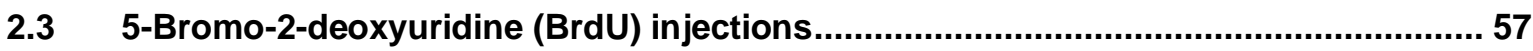

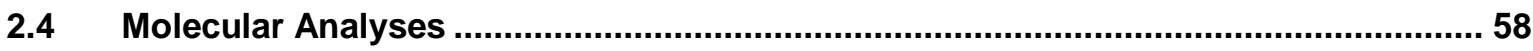

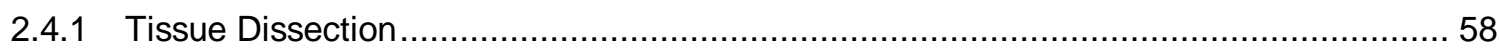

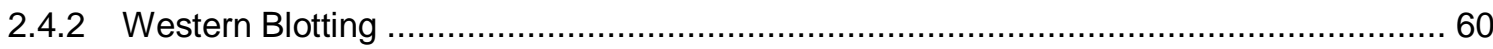

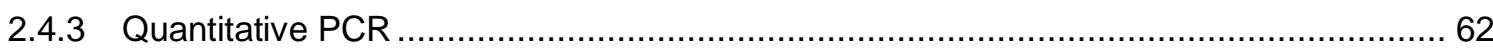

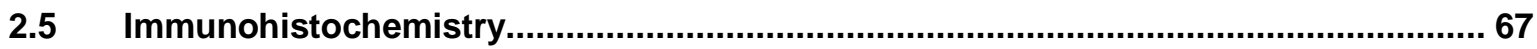

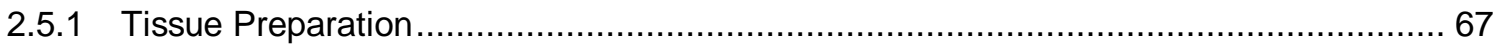

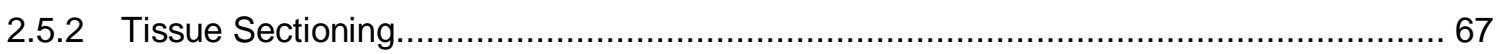

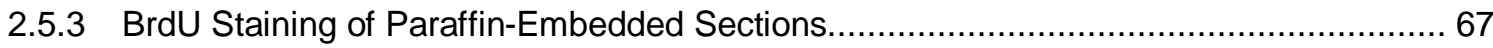

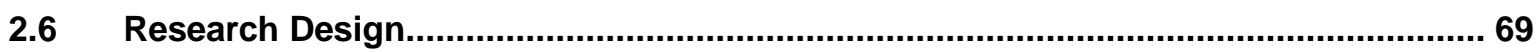

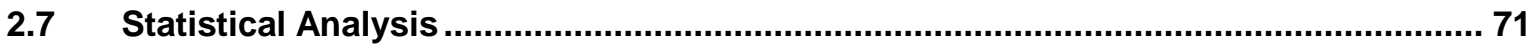

CHAPTER 3 EFFECTS OF THC ON ADOLESCENT LEARNING .....................72

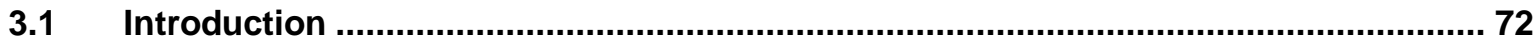

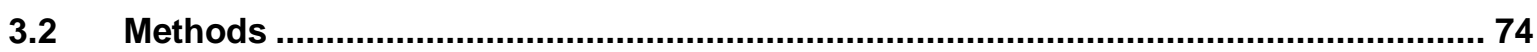

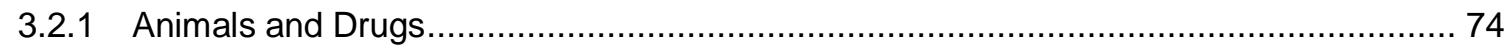

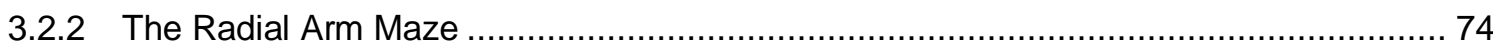




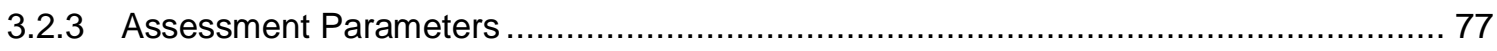

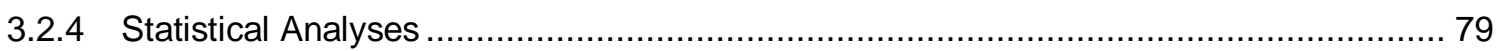

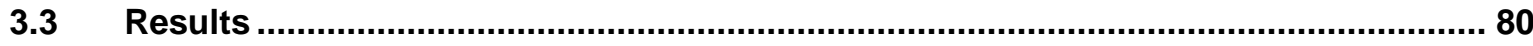

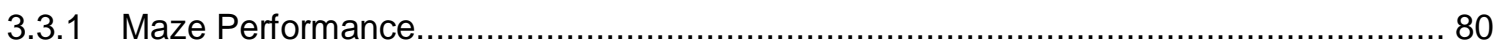

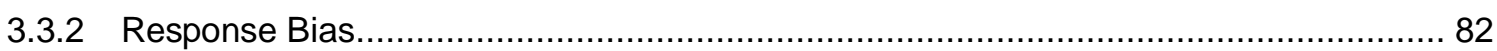

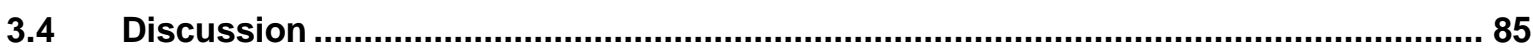

CHAPTER 4 EFFECTS OF THC ON CHANGES IN SYNAPTIC PLASTICITY ...92

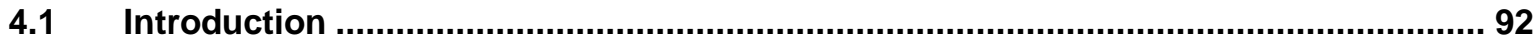

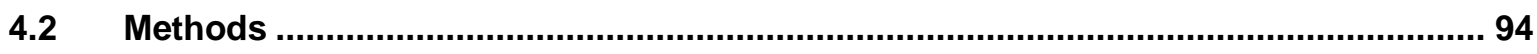

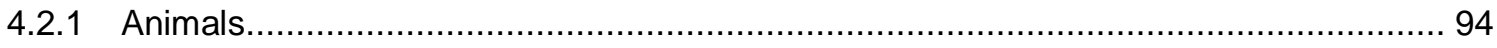

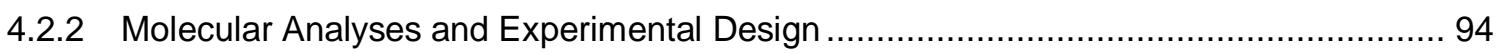

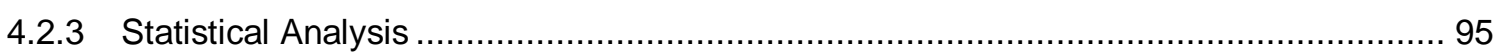

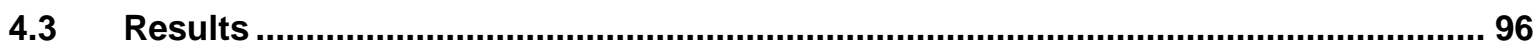

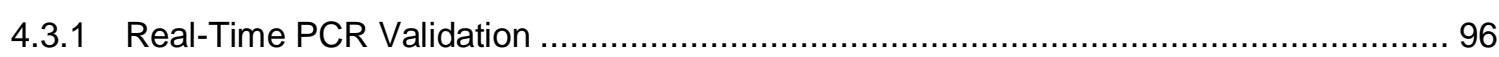

4.3.2 Chronic THC Exposure Attenuates Transcription of Plasticity Markers....................... 100

4.3.3 Chronic THC Exposure Prevents Adaptive Reponses in Synaptic Plasticity ................. 102

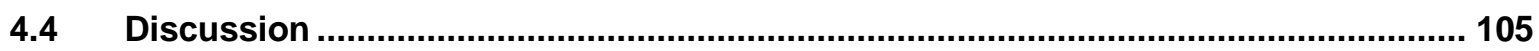

CHAPTER 5 EFFECTS OF THC ON HIPPOCAMPAL NEUROGENESIS..........112

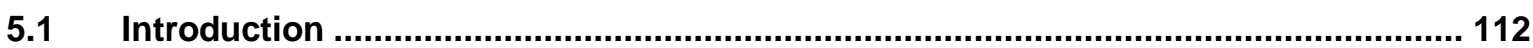

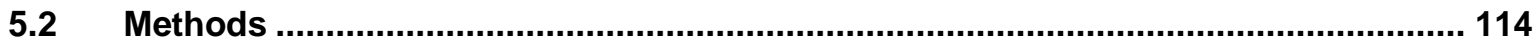

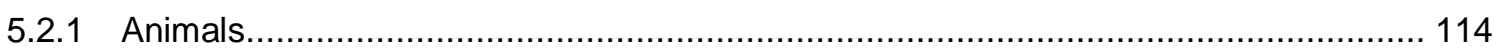

5.2.2 Molecular Analyses and Experimental Design .................................................... 115

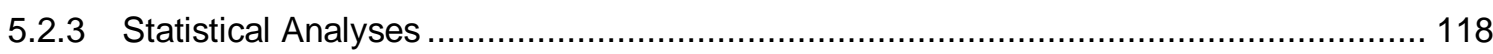

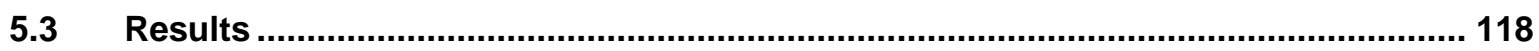

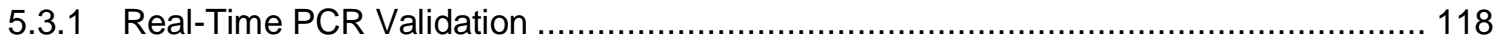

5.3.2 THC Does Not Affect Enhanced Neuronal Survival Associated with Learning in a Cohort

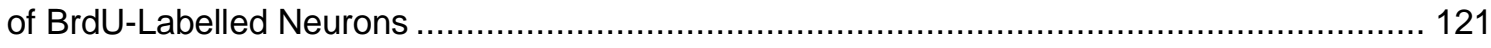

5.3.3 Neurogenesis is Affected by Learning, and THC has Only Subtle Effects .................. 123

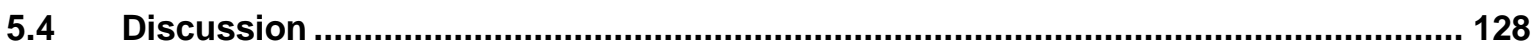

CHAPTER 6 GENERAL DISCUSSION.......................................................136 


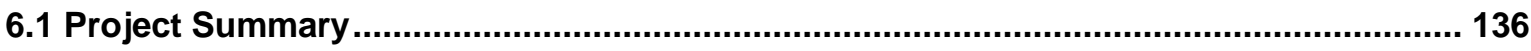

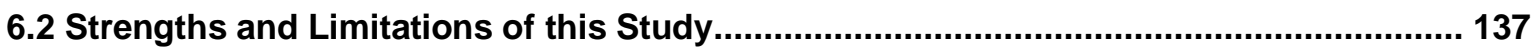

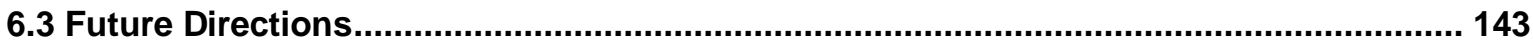

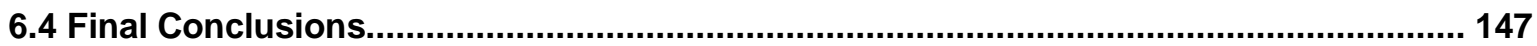

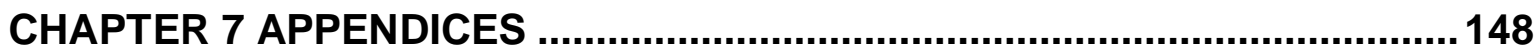

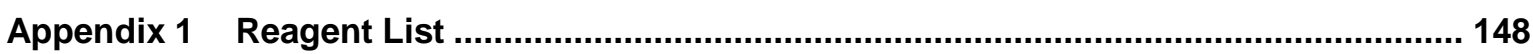

Appendix 2 Hematoxylin staining of brain sections following DAB staining .................... 153

Appendix 3 Considerations of the Effect of Animal Treatment on Learning..................... 154

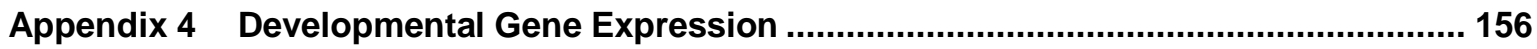

Appendix 5 THC and the Renin-Angiotensin System................................................ 159

Appendix 6 Whole Gel Images of Western-blots ....................................................... 164

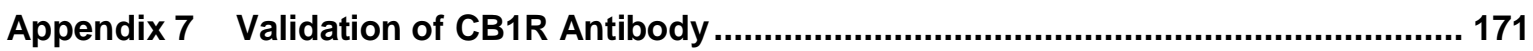

CHAPTER 8 REFERENCE LIST .................................................................... 173 


\section{List of Figures}

Figure 1: Structures of four compounds of Cannabis sativa ......................................

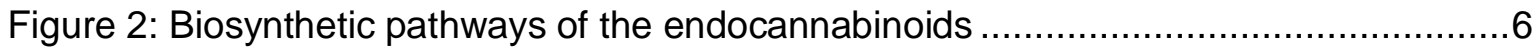

Figure 3: Functional roles of endocannabinoid signalling …...................................... 14

Figure 4: Neural connectivity of the temporal lobe ..............................................29

Figure 5: Development of newborn neurons in the hippocampus ..................................37

Figure 6: The role of neurogenesis in pattern separation in the hippocampus.................40

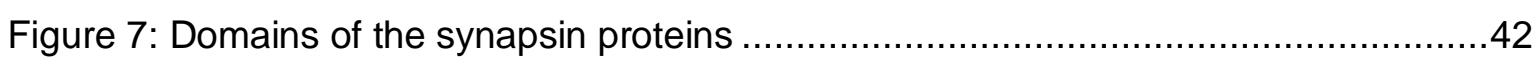

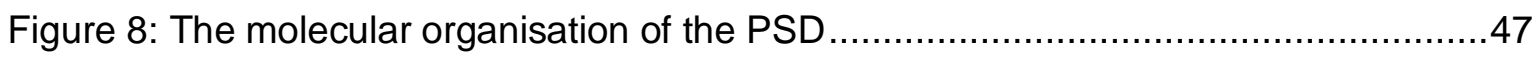

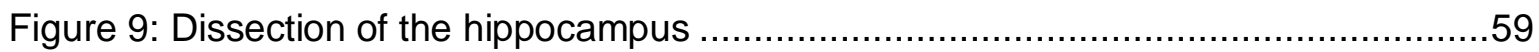

Figure 10: Training schedule and timing of treatments and analyses used in this study ...70

Figure 11: Configuration of the radial maze ........................................................

Figure 12: THC impaired learning in the radial maze .............................................. 81

Figure 13: Chunking facilitates increased performance in vehicle-treated, but not THCtreated animals....

Figure 14: THC-treated animals rely less on spatial searching strategies ......................84

Figure 15: Efficiencies of the primers used to assess markers of plasticity ......................97

Figure 16: Representative real-time PCR data used to assess plasticity........................99

Figure 17: THC attenuates the transcription of markers involved in synaptic plasticity ...102

Figure 18: The training-induced reduction in synaptic protein abundance is blocked by

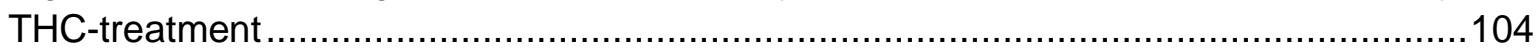

Figure 19: Method for the enumeration of $\mathrm{BrdU}^{+}$nuclei in the hippocampus .................117

Figure 20: Efficiencies of the primers used to assess markers of neurogenesis............119

Figure 21: Representative real-time PCR data used to assess neurogenesis ...............120

Figure 22: THC does not affect the number or localisation of surviving BrdU-labelled neurons

Figure 23: Training in the radial maze reduced mRNA for neurogenic markers, but was unaffected by THC 
Figure 24: Training in the radial maze reduces protein levels of neurogenic markers, and THC has a subtle effect

Figure 25: THC has subtle effects on hippocampal neurogenesis 135

Figure 26: Considerations of the effects of animal treatment on learning 155

Figure 27: The expression of transcripts involved in synaptic plasticity is developmentally regulated.

Figure 28: Efficiencies of the primers used to assess markers of the RAS 160

Figure 29: Representative real-time PCR data used to assess the RAS 161

Figure 30: Neither training nor drug treatment had any significant effect on the expression of genes of the RAS. 163

Figure 31: Western-blot of CB1R 164

Figure 32: Western-blot of PSA-NCAM 165

Figure 33: Western-blot of Sox2 166

Figure 34: Western-blot of BDNF 167

Figure 35: Western-blot of Synapsin-I. 168

Figure 36: Western-blot of Ki67 169

Figure 37: Western-blot of PSD95 170

Figure 38: Validation of CB1R antibody 


\section{List of Tables}

Table 1: Pharmacological effects of cannabinoids in adult and adolescent animals.

Table 2: Primary antibodies used for Western blotting.

Table 3: Secondary antibodies used in Western blotting. Antibodies were either conjugated to Alexa-488 or Alexa-555 dyes

Table 4: Primers used for qRT-PCR analysis. 66

Table 5: Relative gene expression levels between treatment and behavioural groups for plasticity markers

Table 6: Number of BrdU labelled neurons surviving for 2 weeks

Table 7: Relative gene expression levels between treatment and behavioural groups for neurogenic markers

Table 8:Relative gene expression levels between developmental stages for plasticity markers 158

Table 9: Relative gene expression levels between treatment and behavioural groups for markers of the RAS 


\section{List of Commonly Used Abbreviations}

\begin{tabular}{|c|c|}
\hline 2-AG & 2-Arachidonylglycerol \\
\hline ACE & Angiotensin converting enzyme \\
\hline AM251 & $\begin{array}{l}\text { 1-(2,4-Dichlorophenyl)-5-(4-iodophenyl)-4-methyl-N-(1- } \\
\text { piperidyl)pyrazole-3-carboxamide }\end{array}$ \\
\hline AMPA & a-Amino-3-hydroxy-5-methyl-4-isoxazolepropionic acid \\
\hline ANA & Anandamide \\
\hline Ang & Angiotensin \\
\hline AT2R & Angiotensin 2 receptor \\
\hline BDNF & Brain derived neurotrophic factor \\
\hline BrdU & 5-Bromo-2-deoxyuridine \\
\hline CA1 & Cornu ammonis field 1 \\
\hline CA3 & Cornu ammonis field 3 \\
\hline CB1R & Cannabinoid receptor 1 \\
\hline CB2R & Cannabinoid receptor 2 \\
\hline CNS & Central nervous system \\
\hline CP 55,940 & $\begin{array}{l}\text { 2-[(1R,2R,5R)-5-hydroxy-2-(3-hydroxypropyl) cyclohexyl]-5-(2- } \\
\text { methyloctan-2-yl)phenol }\end{array}$ \\
\hline DCX & Doublecortin \\
\hline DG & Dentate gyrus \\
\hline DMTS & Delayed matched to sample \\
\hline DNMS & Delayed non-matched to sample \\
\hline
\end{tabular}


DSE

DSI

EPSCs

FAAH

GABA

GCL

HU-210

IHC

IPSCs

Ki67

LTD

LTP

ML

mRNA

MWM

NMDA

NSC

PCR

PSA-NCAM

PSD

PSD95
Depolarisation-induced suppression of excitation

Depolarisation-induced suppression of inhibition

Excitatory postsynaptic currents

Fatty acid amide hydrolase

Gamma-aminobutyric acid

Granule cell layer

(6aR,10a $R)$ - 9-(Hydroxymethyl)- 6,6-dimethyl- 3-(2methyloctan-2-yl)- 6a,7,10,10a-tetrahydrobenzo [c]chromen1 -ol

Immunohistochemistry

Inhibitory postsynaptic currents

A specific marker of proliferative cells

Long-term depression

Long-term potentiation

Molecular layer

Messenger ribonucleic acid

Morris water maze

$\mathrm{N}$-methyl-D-aspartate

Neural Stem Cell

Polymerase chain reaction

Polysialic acid - neural cell adhesion molecule

Postsynaptic density

Postsynaptic density protein 95 
RAM

RAS

SEM

Sox2

SR141716A

SVZ

THC

VR1

WIN 55,212-2
Radial arm maze

Renin-angiotensin system

Standard error of the mean

SRY-box 2

5-(4-Chlorophenyl)-1-(2,4-dichloro-phenyl)-4-methyl- $N$ (piperidin-1-yl)-1H-pyrazole-3-carboxamide

Subventricular zone

$\Delta^{9}$-Tetrahydrocannabinol

Vanilloid receptor 1

(R)-(+)-[2,3-Dihydro-5-methyl-3-(4-

morpholinylmethyl)pyrrolo[1,2,3-de]-1,4-benzoxazin-6-yl]-1napthalenylmethanon 


\section{Chapter 1}

\section{Introduction}

\subsection{Cannabis Use: Historical Aspects and the New Zealand Perspective}

The effects of cannabis have been known for over 4,000 years with the oldest known pharmacopoeia (the Pen-ts'ao Ching, 2727 BC) describing the medicinal and psychiatric side effects of cannabis. The use of cannabis as a therapeutic agent lost favour as the $20^{\text {th }}$ Century progressed, although recreational use became commonplace to the point where cannabis use today is perceived as a culturally acceptable activity (Murray, Morrison et al. 2007). In New Zealand, the use, supply or possession of cannabis is illegal under the Misuse of Drugs Act 1975, although a 2003 survey by the Ministry of Health revealed that $44 \%$ of New Zealanders have used cannabis in their lifetime, with $16 \%$ classified as frequent users (defined as using cannabis 10 or more times a month over the last 12 months) (Field and Casswell 2000; 2003). A study of 140 New Zealand adolescents in the Canterbury region between the ages of 13 - 18 found that $89 \%$ had tried cannabis and $70 \%$ had tried it more than 5 times in one month at some stage. The median age of initiation was 13 years, and the heaviest use was reported at age 15 years (Harvey, Sellman et al. 2007). Maori were significantly more likely to have used cannabis than non-Maori (2003).

The social costs of illicit drug use can be measured as costs borne by individuals and wider society for which there is no corresponding benefit. The tangible costs of illicit drug use are associated with the diversion of resources due to the presence of illicit drugs (e.g. lost production and the cost of drug associated crime). In addition, intangible costs are borne by illicit drug using individuals (e.g. suffering caused by illicit drug use) and individuals associated with drug users (e.g. suffering caused to the family of an illicit drug user). During the 2005/2006 period the estimated social cost of illicit drug use in New Zealand was \$1.31 billion, of which $\$ 431$ million was attributable to cannabis use (Slack, O'Dea et al. 2008). Fergusson and Boden (2008) assessed the cannabis behaviour of 1256 
New Zealand born children and assessed a range of life outcomes at 25 years of age. Poorer educational achievement, lower income, greater dependency on social welfare, higher unemployment and lower life satisfaction were correlated with higher levels of cannabis use from ages $14-21$ years, even when extensively controlled for covariate factors.

\subsection{Molecular Biology and Biochemistry of the Cannabinoid System}

\subsubsection{Molecular Biology of Cannabinoid Receptors}

Following the identification of $\Delta^{9}$-tetrahydrocannabinol (THC) as the major active component cannabis in 1964 (Gaoni and Mechoulam 1964), and prior to the identification of the cannabinoid receptor nearly a quarter century later, many believed that THC exerted its cognitive effects by non-specifically modifying cell membrane fluidity due to the hydrophobic structure of THC (Trezza, Cuomo et al. 2008). The seven transmembrane $G$ protein-coupled $C B 1 R$ receptor was initially described in 1987 (Bonner, Buckley et al. 1987), although at this point its ligands were unknown. These were subsequently discovered to belong to the cannabinoid family in 1988, using the tritiated cannabinoid agonist CP-55,940 and competitive binding assays in the brain (Devane, Dysarz et al. 1988), and confirmed in 1990 when the $55 \mathrm{kDa}$ CB1R was cloned from rat cerebral cortex (Matsuda, Lolait et al. 1990).

CB1R is functionally coupled to $G_{i}$ proteins. Activation of $C B 1 R$ results in an inhibition of cAMP production by adenylate cyclase (Howlett and Fleming 1984), and inhibition of voltage gated $\mathrm{Ca}^{2+}$ channels (Mackie and Hille 1992; Mackie, Lai et al. 1995; Twitchell, Brown et al. 1997) required for neurotransmitter release (Luebke, Dunlap et al. 1993; Wheeler, Randall et al. 1994). In addition, inward 
rectifying $\mathrm{K}^{+}$channels are activated, hyperpolarising the neuron (Mackie, Lai et al. 1995). Cumulatively, activation of CB1R reduces the likelihood of neuronal activation, allowing neurons to control their activity as a function of network activity, and in this way modulating synaptic plasticity (Freund, Katona et al. 2003). Indeed CB1R is the most widely expressed G protein in the CNS, with high expression levels in brain areas associated with the cognitive and motor effects of cannabis (Herkenham, Lynn et al. 1990).

The cannabinoid receptor 2 (CB2R) was identified in 1993 from HL-60 promyelocytic leukemic cells by sequence homology to the rat CB1R (Munro, Thomas et al. 1993), and like CB1R, is coupled via inhibitory G proteins to adenylate cyclase (Kaminski 1993). CB2R shares only 44\% homology with CB1R suggesting these receptor subtypes diverged some time ago (Munro, Thomas et al. 1993). The low level of homology between these cannabinoid receptor subtypes is reflected in their differential expression and their different roles throughout the body. CB2R is expressed widely in the periphery, particularly in immune tissues (Munro, Thomas et al. 1993; Freund, Katona et al. 2003), and although not initially detected in the brain, low neuronal levels have since been described (Munro, Thomas et al. 1993; Trezza, Cuomo et al. 2008). CB1R, however, is only detected in the peripheral tissues at low levels, and is predominantly found in neurons of the central and peripheral nervous systems (Freund, Katona et al. 2003).

\subsubsection{Cannabinoid Ligands}

The cannabinoid ligands include the endogenous cannabinoids (endocannabinoids) that naturally occur in the nervous system, the active components of Cannabis sativa (principally THC) and their metabolic derivatives, as well as synthetic analogues of these components. There are two endocannabinoid ligands. The first, arachhidonylethanolamide, was discovered by Devane et al. (1992) and named anandamide (ANA; from 'ananda' meaning bliss, 
and 'amide' from the chemical nature of the compound). A second endocannabinoid, 2-arachidonylglycerol (2-AG), was discovered 3 years later (Mechoulam, Benshabat et al. 1995). Each of the endocannabinoids, ANA and 2$A G$, comprise of an arachidonic acid motif and a polar head group of either ethanolamine (ANA) or glycerol (2-AG) (Freund, Katona et al. 2003). The endocannabinoids are synthesised at postsynaptic sites and in immune tissues. In neurons endocannabinoids are synthesised in an activity-dependent manner. The binding of neurotransmitters to receptors, or membrane depolarisation is sufficient to elicit their synthesis. The interaction of these two methods of endocannabinoid synthesis have furthermore been shown to synergistic (Heifets and Castillo 2009).

In the biosynthetic pathway of ANA (Fig. 2eA), an arachidonate group of a phospholipid and phosphatidylethanolamine (PE) undergo a condensation reaction catalysed by $\mathrm{N}$-acyl transferase yielding the ANA precursor $\mathrm{N}$-arachidonoyl PE. Phospholipase D (PLD) then catalyses the hydrolysis of this molecule to ANA. The biosynthesis of 2-AG may proceed in two ways (Fig. 2B). Phosphoinositides (PI) are converted to either 1,2-diacylglycerol (DAG) by phospholipase C (PLC), or to lysophosphoinositides (lyso-PI) by phospholipase $A_{1}\left(P L A_{1}\right)$. These intermediates are then converted to 2-AG by diacylglycerol lipase (DGL) and lyso-PLC, respectively (Freund, Katona et al. 2003). It is believed than the PLC/DAG pathway largely accounts for the biosynthesis of 2-AG in the CNS (Stella, Schweitzer et al. 1997). The synthesis of ANA and 2-AG are $\mathrm{Ca}^{2+}$-dependent and are activated as a consequence of the activation of neurotransmitter receptors (Freund, Katona et al. 2003). 2-AG is present in the brain at levels 170 fold greater than ANA, and activates CB1R as a full agonist, rather than with a partial agonistic activity as seen with ANA (Devane, Hanus et al. 1992; Stella, Schweitzer et al. 1997; Freund, Katona et al. 2003). As lipids, neither ANA nor 2-AG require vesicular release, diffusing directly through the cell membrane to activate presynaptic CB1Rs (Freund, Katona et al. 2003). 


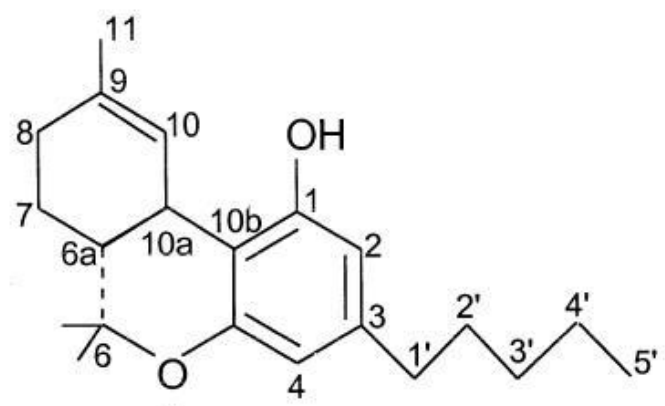

5

$\Delta^{9}$-Tetrahydrocannabinol<smiles>CCCCCc1cc(O)c2c(c1)OC(C)(C)c1ccc(C)cc1-2</smiles>

Cannabinol<smiles>CCCCCc1cc(O)c2c(c1)OC(C)(C)C1CC=C(C)CC21</smiles>

$\Delta^{8}$-Tetrahydrocannabinol

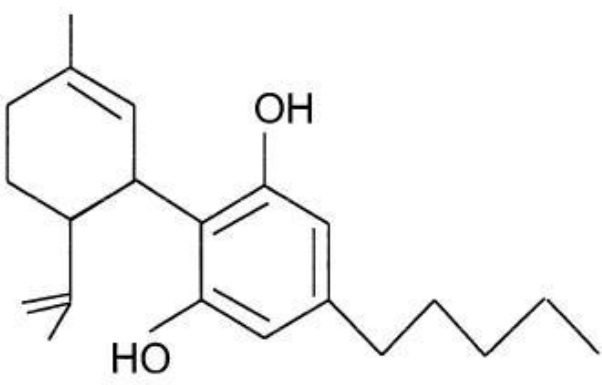

Cannabidiol

Figure 1: Structures of four compounds of Cannabis sativa: the major psychoactive component $\Delta^{9}$-tetrahydrocannabinol, as well as the minor psychoactive compounds $\Delta^{8}$ tetrahydrocannabinol and cannabinol, as well as the non-psychoactive cannabidiol. Adapted from (Ameri 1999).

Two mechanisms mediate the termination of endocannabinoid signalling. A putative transporter facilitates the diffusion of ANA and 2-AG into the cell in a competitive fashion, as evidenced by the fact that the endocannabinoids compete with each other for transport and neither is transported in the presence of a transport inhibitor (Beltramo and Piomelli 2000; Bisogno, Maccarrone et al. 2001; Freund, Katona et al. 2003). The ligands are also rapidly degraded by enzymes. Fatty acid amide hydrolase (FAAH) and monoglyceride lipase (MGL) are abundantly expressed and are located on the cellular inner membranes in close association with CB1Rs (Freund, Katona et al. 2003). ANA is rapidly hydrolysed by 

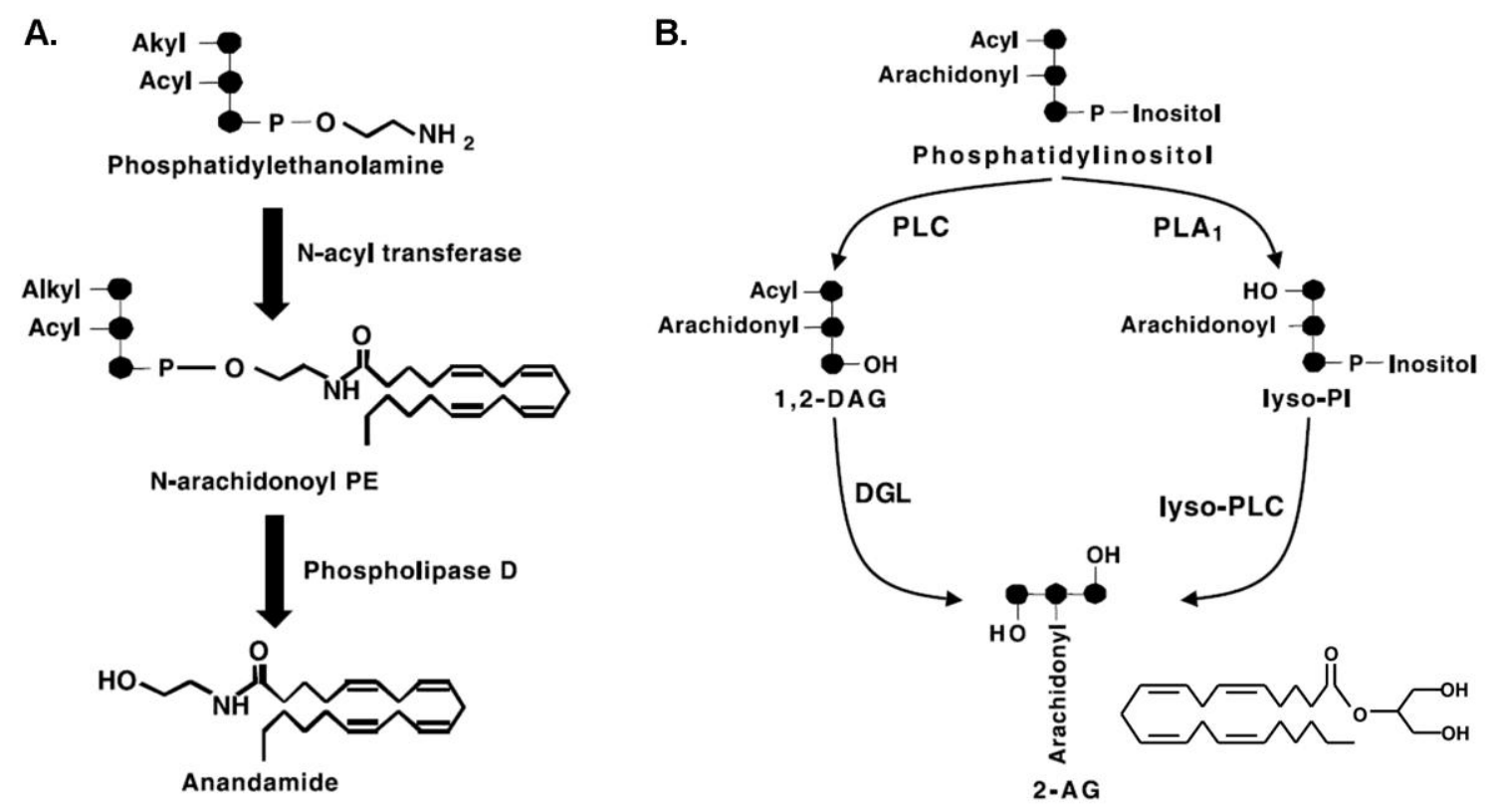

Figure 2: Biosynthetic pathways of the endocannabinoids. A) Biosynthesis of anandamide. Nacyl transferase catalyses the transfer of an arachidonate group of a phospholipid (not shown) to the primary amino group of phosphatidylethanolamine (PE). Phospholipase D hydrolyses the product of this reaction, $\mathrm{N}$-arachidonoyl $\mathrm{PE}$, to anandamide. B) Biosynthesis of 2-arichdonylglycerol (2-AG). Phosphoinositides (PI) are converted into either 1,2 diacylglycerol (DAG) by phospholipase C (PLC), or to lyso-PI by phospholipase A1 (PLA1). These intermediates are converted to 2-AG by diacylglycerol lipase (DGL) and lyso-PLC, respectively. Modified from Freund et al. (2003).

FAAH (Freund, Katona et al. 2003) while 2-AG is primarily degraded by MGL (Beltramo and Piomelli 2000; Lichtman, Hawkins et al. 2002).

\subsubsection{Interactions with Non-Cannabinoid Receptors}

The actions of the CB1 receptor have been strongly linked to the endogenous opioid system (Robledo, Berrendero et al. 2008), which is itself involved in a wide variety of neuronal functions (Wang, Dow-Edwards et al. 2006). Cannabinoids and opioids share a common pharmacological profile, each inducing analgesia, hypothermia, hypotension and immunosuppression (Vigano, Rubino et al. 2005). Furthermore, both opioid and cannabinoid receptors are coupled to adenylate 
cyclase through Gi/Go GTP binding proteins, block voltage gated $\mathrm{Ca}^{2+}$ channels, and activate $\mathrm{K}^{+}$channels as well as the MAP kinase pathway (Childers, Fleming et al. 1992; Howlett 1995). Antagonists of one receptor are able to block the actions of agonists of the other, while co-administration of agonists results in synergistic effects (Howlett 1995). Many of these effects reflect a physiological coupling or receptors, converging at the level of adenylate cyclase, although a demonstration of physical association between these receptor types has still to be achieved (Trezza, Cuomo et al. 2008).

The potential for crosstalk between the cannabinoid and opioid systems in relation to learning and memory has been demonstrated by several biochemical and some behavioural studies. Co-expression of cannabinoid and opioid receptors on the same cell has been demonstrated in the hippocampus, particularly on inhibitory GABAergic interneurons critical for the induction of longterm potentiation (LTP), although co-expression has also been seen in other areas involved in learning and memory. Comparatively little is known about how the interaction of the cannabinoid and opioid systems influences behaviour. The few studies that have been published suggest that the opioid receptor antagonist naltrexone reverses the learning impairment of cannabinoids and vice versa. Taken together, these data certainly appear to indicate that the molecular similarities of the cannabinoid and opioid systems carry over, at least to some extent, to cognitive processes such as learning and memory (reviewed in (Robledo, Berrendero et al. 2008)). Indeed cannabinoid-induced acetylcholine release in the prefrontal cortex and hippocampus, implicated in hippocampal synaptic plasticity which is important for learning and memory, is modulated by the $\mu$-opioid receptor (MOR) (Pisanu, Acquas et al. 2006). Furthermore, cognitive defects in later life attributed to cannabis exposure during development, which include inattention, hyperactivity and depressive symptoms, are believed to be in part caused by cannabinoid-mediated influences on the opioid neurotransmitter system (Sundram 2006). 
In addition to the opioid receptors, there is also strong evidence to suggest that cannabinoids can interact with the vanilloid receptor family to modulate synaptic plasticity. This is discussed in the next section.

\subsection{Roles of the Cannabinoid System}

\subsubsection{In the Periphery}

Cannabinoids, including THC, mediate anti-inflammatory and immunosuppressive effects as well as perturb cognition, analgesia and orexigenic (stimulating the appetite) processes (Dewey 1986). The effects of cannabinoids on the immune system are largely attributable to activation of CB2R, which is enriched in immune tissues (Galiegue, Mary et al. 1995) and is present in peripheral tissues at levels of up to 100 fold that of CB1R (Galiegue, Mary et al. 1995). In addition, immune cells are capable of synthesizing and degrading cannabinoids, suggesting a fully functional autocrine or paracrine signalling system (Greineisen and Turner 2010). The specific effects of cannabinoids on immune tissues include the inhibition of proliferation (Pross, Klein et al. 1987) and cytotoxicity of T lymphocytes (Klein, Kawakami et al. 1991), as well as inhibition of antibody synthesis (Arata, Klein et al. 1991) and interferon production (Blanchard, Newton et al. 1986). Overall, the peripheral endocannabinoids appear to be potent immunomodulatory agents (Greineisen and Turner 2010).

\subsubsection{In the CNS}

The major role of the endocannabinoid system in the central nervous system (CNS) is the regulation of synaptic transmission. This is primarily achieved by the action of the endocannabinoid system in regulating the mechanisms of LTP (Heifets and Castillo 2009). LTP describes the activity-driven strengthening of synapses that is required for learning and memory (Martin, Grimwood et al. 2000). 
Activation of the cannabinoid system by exogenous agents such as THC may reduce neurotransmitter release to levels below that required for LTP (Sullivan 2000).

In the rodent hippocampus, CB1R has been localised to the presynaptic terminal of cholecystokinin (CCK) containing GABAergic inhibitory interneurons, but not the postsynaptic membrane of the synapse (Katona, Sperlagh et al. 1999). The binding of GABA results in inhibitory postsynaptic currents (IPSCs) and a slight hyperpolarisation of the postsynaptic membrane. These IPSCs can be reduced by depolarisation-induced suppression of inhibition (DSI), a $\mathrm{Ca}^{2+}$ dependent mechanism by which presynaptic GABA release is suppressed (Llano, Leresche et al. 1991; Pitler and Alger 1992; Alger and Pitler 1995). The presence of CB1R in the presynaptic membrane suggests that the endocannabinoid system may be important in mediating DSI. The activation of interneurons in the hippocampus by the synthetic cannabinoid agonist WIN 55,212-2 reduced GABA release (Katona, Sperlagh et al. 1999) and IPSCs (Hajos, Katona et al. 2000), and these effects were sensitive to the cannabinoid antagonist SR141716A. CCK-containing GABAergic interneurons are hippocampal basket cells thought to be important in the synchronisation of neuronal activity from large groups of excitatory pyramidal cells (Cobb, Buhl et al. 1995). The association between CCK expressing interneurons and $\mathrm{CB} 1 \mathrm{R}$ extends to many other forebrain areas as well, including the neocortex, amygdala and prefrontal cortex (Ferraro, Tomasini et al. 2001; Katona, Rancz et al. 2001; Freund, Katona et al. 2003), and these associations are preserved in humans (Katona, Sperlagh et al. 2000). The strong association between CB1R and GABAergic neurons in many regions of the CNS (Tsou, Brown et al. 1998) predicts that the endocannabinoid system may play an important role in the regulation of local network activity (Freund, Katona et al. 2003). Thus, through the DSI mechanism, endocannabinoids are able to elicit long-term depression (LTD) at GABAergic inhibitory synapses (Chevaleyre and Castillo 2004). 
As GABAergic tone is able to induce IPSCs and is modulated by DSI, glutamatergic neurotransmission evokes excitatory post synaptic currents (EPSCs - characterised by a slight depolarisation of the postsynaptic membrane), and these are modulated by depolarisation-induced suppression of excitation (DSE) (Kreitzer and Regehr 2001). DSE is a $\mathrm{Ca}^{2+}$-dependent process that is mediated at the presynaptic neuron and is able to regulate ESPCs by inhibiting excitatory neurotransmission in neurons undergoing LTP (Kreitzer and Regehr 2001). In this way DSE preserves the specific regional activation profile characteristic of LTP by preventing the aberrant activation of neighbouring neurons (Ohno-Shosaku, Tsubokawa et al. 2002), as well as preventing excitotoxicity in these neural pathways (Marsicano, Goodenough et al. 2003; Monory, Massa et al. 2006). DSI, however, enables neurons in the basal state to be activated into LTP when another stimulus is received during disinhibition which would otherwise be unable to induce LTP (Alger and Pitler 1995). Thus it appears that the relative activity of the short-term (tens of seconds) effects of DSI and DSE plays a crucial role in the proper induction and maintenance of LTP (Alger and Pitler 1995; Ohno-Shosaku, Tsubokawa et al. 2002; Freund, Katona et al. 2003). The requirement of an increase in intracellular $\mathrm{Ca}^{2+}$ for both DSI and DSE coincides with the requirement of $\mathrm{Ca}^{2+}$ for the synthesis of endocannabinoids (see section 1.2.2) (Freund, Katona et al. 2003) and both DSI and DSE operate via CB1R receptors (although DSE appears to be approximately 30x less sensitive to cannabinoids) (Ohno-Shosaku, Tsubokawa et al. 2002; Monory, Massa et al. 2006).

The link between endocannabinoids and DSE occurs despite comparatively little CB1R expression on glutamatergic neurons (Tsou, Brown et al. 1998). CB1R does appear to be involved however, as ESPCs are sensitive to the cannabinoid agonist WIN 55,212-2, the effect of which can be antagonised by SR141716A (Shen, Piser et al. 1996; Ameri, Wilhelm et al. 1999; Misner and Sullivan 1999; Auclair, Otani et al. 2000). However cannabinoids evoked ESPCs have also been reported to be unaffected in $\mathrm{CB}_{1} \mathrm{R}^{-/}$mice (Hajos, Ledent et al. 2001), can be antagonised by the vanilloid antagonist capsazepine, and can mimicked by the vanilloid agonist capsaicin (Hajos and Freund 2002) suggesting that a molecularly distinct receptor sensitive to cannabinoids participates in DSE. This receptor may be the vanilloid 
receptor 1 (VR1) since the synthetic cannabinoid antagonist SR171614A has subsequently been shown to act at both the CB1R and VR1 receptors (Hajos, Ledent et al. 2001).

A differential regulation of DSI and DSE by endocannabinoids has been proposed. Anandamide is able to bind the VR1 receptor (Zygmunt, Petersson et al. 1999) and inhibit EPSCs (Ameri, Wilhelm et al. 1999), whereas 2-AG does not affect EPSCs despite being present in the brain at levels 170 fold greater than ANA (Stella, Schweitzer et al. 1997). DSI however relies on 2-AG only, thus ANA and 2AG differentially modulate DSI and DSE respectively (Freund, Katona et al. 2003). They may also be a functional interaction between the two endocannabinoids. Kainic acid induces a massive increase in ANA levels to prevent seizures in a CB1R dependent mechanism (Monory, Massa et al. 2006), and artificial enhancement of ANA levels reduces DSI and 2-AG levels (Maccarrone, Rossi et al. 2008). Furthermore, VR1 mediates the internalisation of AMPA receptors in the postsynaptic neuron upon ANA binding, leading to LTD (Chavez, Chiu et al. 2010). Thus, increased ANA production during times of high neural activity appears to regulate neurotransmission be re-establishing GABAergic transmission through a reduction of 2-AG production, and the removal of AMPA-type glutamate receptors. Such a mechanism may help explain the biphasic effects of cannabinoids on glutamate release, where low concentrations enhance EPSCs while inhibition of EPSCs is observed with concentrations an order of magnitude larger (Kujtan, Carlen et al. 1983; Nowicky, Teyler et al. 1987; Kirby, Hampson et al. 1995; Freund, Katona et al. 2003). The biphasic sensitivity of DSI and DSE to cannabinoids may in part reflect the involvement of multiple receptor systems in DSE, and the functional interaction of the endocannabinoids.

Owing to the integral role of the endocannabinoid system in mediating LTP, it is hardly surprising that exogenous cannabinoids are able to perturb cognitive processes. The behavioural impairment resulting from cannabinoid exposure is discussed in Section 1.4. 


\subsubsection{Emerging Roles}

In addition to the relatively well defined role of endocannabinoids in the regulation of LTP/LTD by retrograde signalling, evidence is also emerging that the endocannabinoids may act as paracrine and autocrine regulators (Fig. 3). This subject has been reviewed by Ohno-Shosaku et al (2011).

While CB1Rs are generally thought of as regulating GABAergic transmission at presynaptic sites only, there also appears to be some somatodendritic CB1 $R$ expression on inhibitory interneurons. The release of endocannabinoids from activated glutamatergic neurons appears to decrease the firing rate of adjacent interneurons that, because of their relatively long axons, potentiates the effects of endocannabinoid release beyond the diffusible limits of the ligands (Kreitzer, Carter et al. 2002). Furthermore, a subpopulation of hyperexcitable GABAergic interneurons also appear to regulate their own activity through an 2-AG and CB1R mediated mechanism. Glial cells express CB1R and are capable of synthesising endocannabinoids, suggesting an ability to communicate using the endocannabinoid system. Much as somatodendritic CB1Rs on interneurons are able to potentiate the effect of endocannabinoid release by inducing LTD at distant GABAergic synapses, activation of CB1Rs on astrocytes potentiates the excitatory transmission, possibly by stimulating astrocytic glutamate release, and activating neurons that are again beyond the diffusible limits of the endocannabinoids (Navarrete and Araque 2011).

Finally, cannabinoids may act through non-cannabinoid type receptors. For example ANA may act as an intracellular signalling molecule through its actions on VR1, wherein VR1 activation induces the internalisation of AMPA receptors resulting in LTD in the postsynaptic neuron (Chavez, Chiu et al. 2010). An additional G-protein coupled receptor, GPR55 has been proposed as CB3R because of its structural homology in the binding region, as well as its ability to bind to a number of cannabinoid ligands, although its exact pharmacology remains 
unclear (Moriconi, Cerbara et al. 2010). The emerging roles and components of the cannabinoid system demonstrate the rapidly expanding knowledge, although also the importance of continued study of cannabinoid biology. Regardless, cannabinoid signalling represents a significant modulatory system in the CNS, of which disruption could be expected to lead to a variety on impairments. 


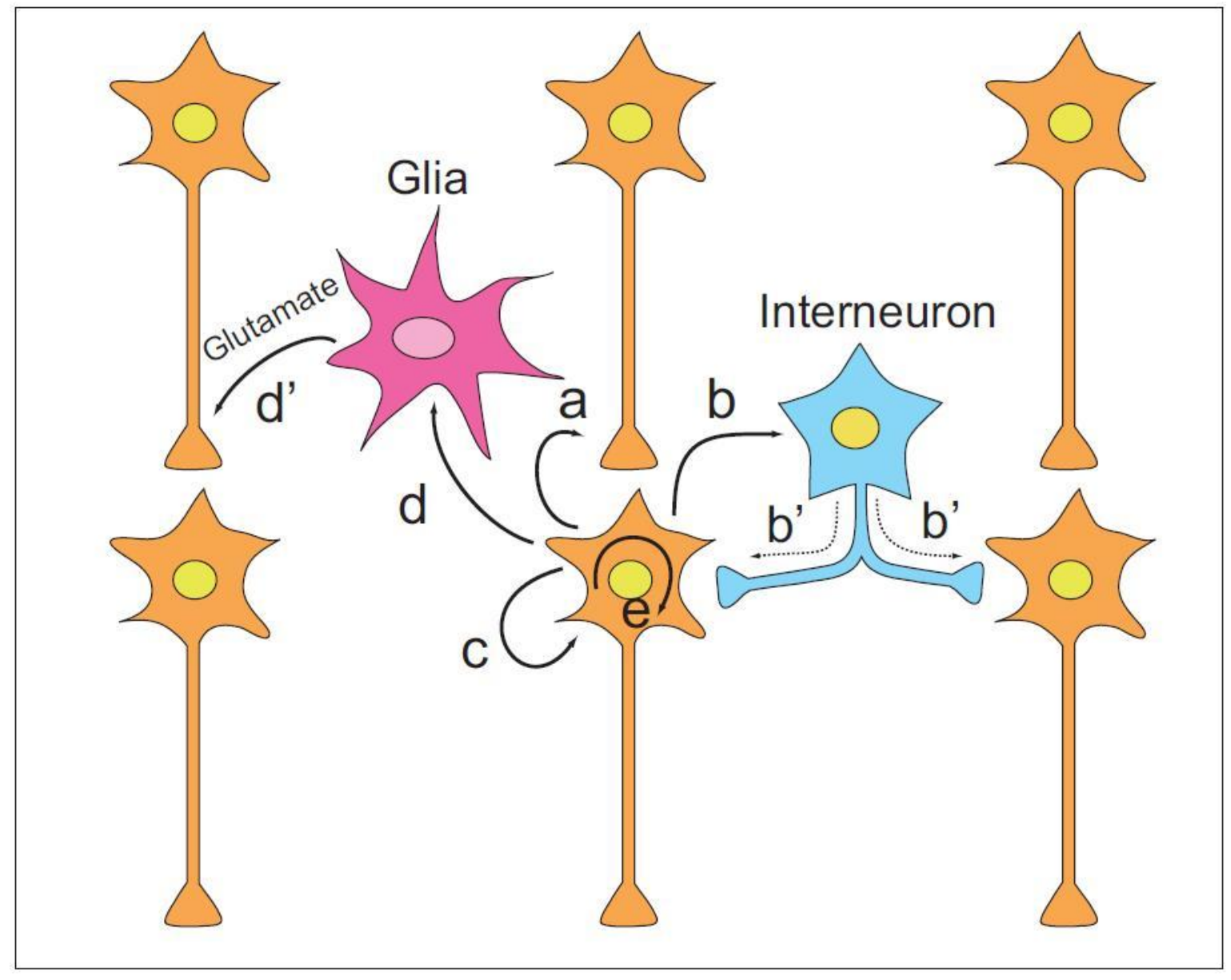

Figure 3: Functional roles of endocannabinoid signalling. Endocannabinoids, released from postsynaptic sites, have a well defined function as retrograde messengers regulating neurotransmitter release (a). In addition, activation of CB1Rs on the soma and dendrites of neighbouring interneurons (b) can potentiate the effect of endocannabinoid release beyond the diffusible limits of the endocannabinoids by regulating GABA release and LTP induction at distant sites (b'). Some hyperexcitable interneurons use their own endocannabinoid release as a means of regulating their activity in an autocrine fashion (c). Activation of CB1Rs on astrocytes by endocannabinoids released during neuronal excitation (d) can result in glutamate release and the excitation of neurons at sites beyond the diffusible limits of the endocannabinoids ( $d$ '). The endocannabinoid ANA acts as an intracellular signalling molecule via VR1 at some postsynaptic site, regulating LTD by the internalisation of AMPA receptors (e). Image adapted from (OhnoShosaku, Tanimura et al. 2011) 


\subsection{Behavioural Consequences of Cannabinoid Exposure}

In humans the use of cannabis peaks between the ages of 15 and 30 years, with a trend of continued use from $30-40$ years emerging (Trezza, Cuomo et al. 2008). A range of cognitive impairments are associated with cannabis abuse in humans, including psychomotor, analgesic, satiety and perceptual effects, as well as deficits in learning and memory performance across a wide range of tasks. Many of these effects are also observed in rodent models (Iversen 2003).

\subsubsection{Methods of Behavioural Assessment}

Measurements of spatial learning and memory have commonly been used to investigate the effects of physical and chemical lesions on learning and memory processes. Memory is classically categorised into two major groups. Declarative memory is related to information that is communicated or expressed, and procedural memory is related to information about procedural or motor skills which is not communicated. In addition, a distinction between long- and short-term memory is commonly drawn. Spatial memory cannot be attributed to any one memory category but is thought to involve contributions from several memory processes. Spatial memory uses cues from the environment to locate positions in space, and is likely to have evolved as a means of preservation and survival of a species. It allows individuals to locate food while avoiding adverse situations based on past experiences (Paul, Magda et al. 2009). Spatial memory is therefore a collection of processes responsible for recognising, coding, storing and recovering information about the spatial arrangement of objects or routes in space (Kessels, de Haan et al. 2001).

Mazes such as the radial arm maze (RAM) and Morris water maze (MWM) are typically used in the assessment of spatial learning and memory (Paul, Magda et al. 2009). Both working and reference memory are involved in each of these tasks. 
In the initial description of the RAM, animals were required to find food deposited at the end of each of 8 arms radiating from a central platform. In order to maximise performance in this task, animals must remember from which arms they have retrieved food and to which arms they should visit next (Olton and Samuelson 1976). This design relies heavily on working, or short-term (trial dependent), memory processes with little reference, or long-term (trial independent), memory input. The RAM design has been adapted to allow a subset of the arms to be baited, requiring the animals to learn which arms contain rewards (reference memory) and which arms have already been investigated (working memory). Learning is inferred from the accuracy with which animals enter the arms containing food over successive trials, and errors are attributed to either working or reference memory errors. The MWM apparatus is a round pool that is filled with opaque water to conceal a platform located $2-3 \mathrm{~cm}$ beneath the water's surface in a consistent location. The latency before animals locate the platform over successive trials is used to assess learning (or acquisition of reference memory), while memory is assessed in a probe trial following the removal (reference memory) or relocation (working memory) of the submerged platform (D'Hooge and De Deyn 2001; Paul, Magda et al. 2009). Spatial reference memory has been reported to be of a greater capacity, duration and resistance to interference than working memory (Olton, Becker et al. 1979; Moscovitch, Rosenbaum et al. 2005; Paul, Magda et al. 2009), although the acquisition of spatial memory over successive trials may be more sensitive to disruption (Da Silva and Takahashi 2002).

While the MWM holds some distinct advantages over the RAM, including a faster rate of learning and no requirement to motivate the desired behaviour by either food or water deprivation (Paul, Magda et al. 2009), the MWM lacks the ability to investigate some executive functions such as chunking. First proposed by Miller (1956), chunking is a strategy of working memory that allows discrete memory traces to be organised into related groups called 'chunks', which can then be recalled as a whole rather than as individual entities. Memories are continuously reorganised into fewer, but larger, chunks until a suitably small number of chunks is achieved to recall all the information accurately (Miller 1956). Chunking has 
been demonstrated in a range of species, including pigeons (Terrace 1987), rats (Dallal and Meck 1990; Cohen, Mallet et al. 1993; Macuda and Roberts 1995; Brown and Terrinoni 1996) and humans (Sakai, Kitaguchi et al. 2003; De Lillo 2004; Ridgeway 2006), suggesting that it may be an important, evolutionarily conserved, process.

Solution strategies in mazes designed to assess spatial learning and memory have been proposed to fall along a continuum, each point of which relies on mnemonic processes to varying degrees. Briefly, animals can be considered to advance along this continuum from random sampling, with little reliance on memory, through response algorithms with limited requirement of mnemonic processes until spatial mapping is achieved, in which strong representations of both reference and working memory are required (Dallal and Meck 1990; Paul, Magda et al. 2009). Chunking is an extension of spatial mapping in which the relationship between spatial goals is organised into groups or 'chunks' to ease the difficulty of the task (Dallal and Meck 1990). This reorganisation may be particularly evident in the RAM. Crucially, Miller describes chunking as a gradual process in which chunk volume is incrementally increased as associations are formed (Miller 1956).

Working memory can additionally be assessed in non-spatial behavioural paradigms. Passive and active avoidance tasks are commonly used to assess working memory in rodents. In the passive avoidance task, a biologically probable response, the movement of an animal from an illuminated to a darkened environment, is associated with a mild foot shock; in the active avoidance task foot shock is preceded by an auditory stimulus, thus allowing the animal an opportunity to escape. An increased latency to enter the darkened environment (passive avoidance), or decreased latency to escape the foot shock (active avoidance), is regarded as a positive correlate of working memory (Jarvik and Kopp 1967; Paul, Magda et al. 2009). These tasks are based on associative learning (Bammer 1982) and require little spatial input, although they have been adapted to include spatial components (Cimadevilla, Kaminsky et al. 2000). Another commonly used 
paradigm in the evaluation of working memory is the novel object recognition task. This task exploits the natural exploratory behaviour of rodents when presented with novel stimuli. Briefly, the task consists of exposing an animal to two objects inside a chamber and allowing it to explore them freely. The animal is then removed for a period of time before being reintroduced to the chamber in which one of the objects has been replaced. Animals with intact working memory investigate the new object preferentially as they remember that they have seen the other object before (Ennaceur and Delacour 1988). A final method for testing working memory using non-spatial methods is the delayed-matched (or nonmatched) to sample task (DMTS or DNMS, respectively). Generally the subject is presented with a sample stimulus, the stimulus is the removed and a period of time is allowed to lapse. The original stimulus plus a novel stimulus is then presented to the subject again. Each of the stimuli is associated with a lever, and the subject indicates which is the original (DMTS) or novel (DNMS) stimulus in order to gain a reward. The accuracy of the subject's performance in this task represents the integrity of working memory (Lichtman, Varvel et al. 2002).

The impairment of working memory by cannabis is supported by studies assessing behaviour in both the spatial and non-spatial domains using methods described above. These studies are discussed in the following sections.

\subsubsection{Consequences of Exposure in Adulthood}

Reports on the effects of cannabinoids on learning and memory largely agree that specific domains are affected while others appear uninhibited. In the context of spatial learning and memory, this is observed by the repeated demonstration that aspects of the tasks relating to short term (working) memory are impaired, while retrieval of information representing long term (reference) memory is intact (Lichtman, Varvel et al. 2002). 
A delayed RAM task has often been used to measure the effect of cannabinoids on spatial working memory in rodents. In this task, animals are allowed to enter a group of arms before being removed from the apparatus for a period of time (predelay). In order to accurately perform the task once they are reintroduced to the apparatus (post-delay) animals must remember which arms were visited predelay. Studies by Lichtman's group (Lichtman, Dimen et al. 1995; Lichtman 2000; Wise, Iredale et al. 2008) utilised a delayed task in the RAM in which seven of eight rewarded arms were available for entry pre-delay. In the post-delay phase all arms were available for entry and animals were required to locate the previously unavailable arm. Their results suggest that cannabinoids affect working memory in the RAM as post-delay performance was impaired by cannabinoids. Similar effects are observed when the number of arms post-delay is increased to four (Nakamura, Dasilva et al. 1991; Molinaholgado, Gonzalez et al. 1995). A limitation of these studies, modelled heavily on the initial description of the RAM by Olton and Samuelson in which every arm contains a food reward (Olton and Samuelson 1976), is that they contain no reference memory component. Therefore, excluding any impairment of reference memory from these studies alone is not possible.

Other studies have assessed the relative impairment of reference and working memory associated with cannabinoid exposure in tasks specifically designed to include both working and reference memory components. Mishima et al. (2001) pre-trained animals to a performance criterion in a working and reference memory task in the RAM. Animals were then treated with THC and their behaviour assessed following treatment. A significant increase in working memory errors was observed in the RAM, while reference memory was unimpaired. Importantly the reference memory component of this task represents the retrieval of learnt information (the retrieval of reference memory). The attainment of reference memory was tested using the MWM and no impairment was observed. This study extends others that have investigated the effect of cannabinoids on memory by demonstrating that spatial working memory, but not reference memory is impaired in either the attainment of retrieval phases. Working, but not reference memory (during attainment phases) has also been shown to be affected by THC in the MWM (Varvel, Hamm et al. 2001). However, a later study by Da Silva and 
Takahashi (2002) used the MWM to show that THC was able to impair not only working memory, but also the attainment of reference memory while retrieval processes were uninhibited. Thus, while spatial working memory certainly appears to be impaired by cannabinoid exposure, reference memory appears to be more robust, although some aspects of reference memory may be more sensitive than others.

A number of investigations of the effect of cannabinoids on working and reference memory in the non-spatial domain support the findings in the RAM and MWM. The DMTS/DNMS tasks have been used to illustrate that the CB1R agonists THC (Heyser, Hampson et al. 1993) and WIN55,212-2 (Hampson and Deadwyler 1999) are able to impair working memory, while cannabinoids also impair working memory assessed in passive avoidance (Castellano, Cabib et al. 1997) and novel object recognition tasks (Barna, Soproni et al. 2007). As these tasks test exclusively working memory, some have paired them with tasks that assess reference memory to better assess the relative impairment of these processes by cannabinoids. Mallet et al. (1996; 1998) showed that cannabinoids impaired working memory in a DNMS task, while reference memory was unimpaired in a non-spatial visual discrimination task. In this task a food reward is associated with entry to a chamber marked with a specific cue and the accuracy with which animals enter the correct chamber represents reference memory. These results have been replicated, in that THC impaired working memory in the passive avoidance paradigm while visual discrimination was not impaired (Mishima, Egashira et al. 2001).

Thus, spatial and non-spatial assessments of the effects of cannabinoids on working and reference memory largely agree. Working memory is consistently impaired by cannabinoids using a variety of paradigms, whereas retrieval of reference memory is not. The attainment of reference memory, however, may be more sensitive to disruption by cannabinoids, and although this remains unclear, adolescents may be more susceptible to this impairment. 


\subsection{The Importance of Adolescence}

Much of the literature that has assessed the behavioural impairment associated with cannabis use has focused on the consequences of adult exposure to this drug, neglecting the adolescent period entirely. While some assess the eventual effects of cannabis exposure during adolescence on adult cognitive processes later in life, there are few studies that assess adolescent cognition concurrent with adolescent cannabis use. This is despite the fact that cannabis is the most widely used illicit drug amongst this age group (Adriani and Laviola 2004), and that increasing cannabis use during adolescence is associated with poorer educational achievement (Fergusson and Boden 2008). Furthermore adolescence is a period of final brain development that underlies the emergence of adult cognition, the disruption of which could be expected to have significant consequences (Spear 2000).

The need for adolescent-specific research is highlighted by the fact that the expression of CB1R peaks during adolescence before declining to adult levels. The higher expression of CB1R during adolescence may render adolescents more susceptible to the effects of cannabinoids by providing more binding sites through which they may act (Spear 2000; Adriani and Laviola 2004). The onset, duration and magnitude of the effects of $\mathrm{CB} 1 \mathrm{R}$ activation by THC are affected by the pharmacokinetics of THC. The absorption, metabolism and elimination of the drug all affect its bioavailability, and these factors may change over time (Huestis 2007). While little is known about how developmental age effects pharmacokinetics (Anderson 2010), differences in pharmacokinetic properties may explain why female rats are more sensitive to some effects of cannabinoids than males. Females have lower overall body fat, providing fewer absorption sites for THC as it is a lipophillic drug thus increasing its bioavailability, as well as an isozyme of cytochrome P450 that produces greater quantities of the psychoactive THC metabolite, 11-hydroxy- $\Delta^{9}$-tetrahydrocannabinol (Tseng, Harding et al. 2004). Lower body fat percentage in adolescent versus adult animals could similarly contribute to greater cognitive impairment in adolescents, whereas activity of 
metabolic enzymes has been shown to be at adult levels by adolescence (Anderson 2010). Nonetheless the potential for the continued maturation of pharmacokinetics during adolescence, and the lack of understanding of these processes, further highlights the need for adolescent-specific research.

\subsubsection{An Important Neurodevelopmental Period}

The adolescent period is generally associated with a loss of synapses and associated structures required for neurotransmission. These processes are proposed to be a result of developmental plasticity in which environment and experience dictate the final cortical architecture. This subject has been reviewed by Spear (2000).

A general overproduction then pruning of synapses takes place during adolescence, which in humans occurs between $7-16$ years of age. By the end of adolescence as many as $50 \%$ of the synapses per neuron are lost in some cortical areas and this coincides with focal, rather than wide-spread activation of the brain during task performance. This reduction in synaptic density is correlated with alterations in neurotransmitter receptors in various brain regions. For example, expression of the NMDA-type glutamate receptor peaks in the prefrontal cortex (PFc) and hippocampus during adolescence, reducing by approximately one third by adulthood (Spear 2000). Furthermore, dendritic spine density on granule cells of the dentate gyrus decreases during adolescence (Zehr, Nichols et al. 2008). Dopaminergic (DAergic) input, dopamine synthesis and expression of the dopamine transporter (DAT) continues to increase in the PFc during adolescence while, in the hippocampus and striatum, these systems peak during adolescence then decline to adult levels. Generally, activation of DAergic systems is greater in adolescence than adulthood, as indicated by the higher levels of the second messenger cAMP in adolescence. Receptors for GABA transmission increase during adolescence in the hippocampus, whereas $C B 1 \mathrm{R}$ expression peaks during adolescence before declining to adult levels. Changes in the expression of 
components of the serotinergic and cholinergic systems have also been reported to occur during adolescence (Spear 2000).

From these studies it is obvious that the relative contribution of the various neurotransmitter systems can be expected to differ between adolescent and adult individuals. As such, the effects of drugs on cognitive effects observed in adult animals cannot necessarily be extrapolated to the adolescent period. Rather, studies that focus on adolescent individuals specifically are required.

\subsubsection{Behavioural Effects of Adolescent Cannabinoid Exposure}

Relatively few studies investigate the adolescent period specifically. In these studies, animals are treated with cannabinoids during adolescence and after a period of abstinence their behaviour is tested in adulthood. These behavioural effects are compared to the effects seen in adult animals that were identically treated during adulthood. Any difference in cognitive abilities is taken to represent the relative sensitivity of adolescence. Using the synthetic cannabinoid agonist CP 55,940 , adolescent animals exhibit greater levels of anxiety and impairment of working memory in the novel object recognition task than adult animals (Schneider and Koch 2003; O'Shea, Singh et al. 2004). Initiation of cannabis use during adolescence, but not adulthood, is also associated with increased levels of depression (Rubino, Vigano et al. 2008), as well as impairment of working memory and strategy selection in the RAM (Rubino, Realini et al. 2009). As a consequence of the research design employed in these studies, requiring periods of treatment and abstinence, the behavioural consequences of adolescent cannabinoid exposure are actually tested in adulthood, not during the adolescent period. Thus, these results suggest that either exposure during adulthood does not affect these behavioural measures as adults exposed during adulthood were unimpaired following a period of abstinence, or more likely that the residual effects of cannabis exposure during adolescence persist longer (Rubino, Vigano et al. 2008; Rubino, Realini et al. 2009). Others have assessed the differences in cognitive 
consequences that an adolescent versus an adult user will experience concurrently while cannabis is being used.

Adolescent animals generally find THC less aversive than adult animals, are equally sensitive to the anxiogenic properties of the drug, and display greater lasting cognitive impairment of working memory in the novel object recognition task later in adolescence after a short abstinence period (Quinn, Matsumoto et al. 2008). The fact that the adolescent brain is still developing may protect these animals from the aversive effects of THC treatment, which may manifest in the human situation as higher rates of continued drug use. These data further demonstrate the relative susceptibility of working memory during the adolescent period (Quinn, Matsumoto et al. 2008). It is important to note that the drug treatment regimen used in this study did not include any drug-seeking component. Therefore, the aforementioned consequence of reduced anxiety and continuing drug use has the potential to augment the reported deficit in working memory in humans.

Fewer studies have assessed the effect of adolescent exposure to cannabinoids on learning concurrent with cannabis use. The nature of this impairment was tested in two studies by Cha et al. $(2006 ; 2007)$ using THC as the cannabinoid agonist. Adult animals treated with THC learnt the task at rates comparable to control animals in the MWM, whereas adolescents were significantly impaired by THC treatment. These data strongly suggest that developmental age plays a significant role in determining the effects of cannabis on learning. 


\begin{tabular}{|c|c|c|c|}
\hline $\begin{array}{c}\text { Ligand and Treatment } \\
\text { Period }\end{array}$ & Behaviours tested & Findings & Reference \\
\hline $\begin{aligned} \text { THC } & \\
\text { - } & \text { During } \\
& \text { adolescence } \\
\text { - } & \text { During adulthood }\end{aligned}$ & $\begin{array}{l}\text { Tests conducted during THC- } \\
\text { treatment (adolescents tested } \\
\text { during adolescence) } \\
\text { - Place aversion } \\
\text { - Anxiety } \\
\text { Tests conducted following } \\
\text { washout (adolescents tested in } \\
\text { early adulthood) } \\
\text { - Working memory }\end{array}$ & $\begin{array}{l}\text { Adult animals found THC adversive during drug } \\
\text { treatment and this persisted into the wash-out } \\
\text { phase, whereas no aversion was seen in adolescent } \\
\text { animals. } \\
\text { THC was equally anxiogenic in adolescent and adult } \\
\text { rats. } \\
\text { Residual effects of THC on working memory were } \\
\text { seen in only adolescent animals. }\end{array}$ & $\begin{array}{l}\text { (Quinn, Matsumoto et } \\
\text { al. 2008) }\end{array}$ \\
\hline $\begin{aligned} \text { THC } & \\
\text { - } & \text { During } \\
& \text { adolescence } \\
\text { - } & \text { During adulthood }\end{aligned}$ & $\begin{array}{l}\text { Spatial learning was compared } \\
\text { in the MWM following: } \\
\text { - Acute THC }(2.5,5, \\
10 \mathrm{mg} / \mathrm{kg})^{*} \\
\text { - Chronic THC following a } \\
\text { washout period (5 } \\
\text { mg/kg) } \\
\text { *Training was during treatment } \\
\text { period } 30 \text { min following } \\
\text { treatment }\end{array}$ & $\begin{array}{l}\text { At all doses, THC impaired spatial learning more in } \\
\text { adolescent than in adult rats. } \\
\text { THC disrupted learning in a dose dependent } \\
\text { manner, with the impairment by } 2.5 \mathrm{mg} / \mathrm{kg} \text { and } 5 \\
\mathrm{mg} / \mathrm{kg} \text { differing significantly in adolescent but not } \\
\text { adult rats. } \\
\text { No lasting impairment of spatial learning was } \\
\text { observed in either adolescent or adult rats. }\end{array}$ & $\begin{array}{l}\text { (Cha, White et al. } \\
2006) \\
\text { (Cha, Jones et al. } \\
\text { 2007) }\end{array}$ \\
\hline
\end{tabular}




\begin{tabular}{|c|c|c|c|}
\hline $\begin{array}{c}\text { Ligand and Treatment } \\
\text { Period }\end{array}$ & Behaviours tested & Findings & Reference \\
\hline $\begin{aligned} \text { THC } & \\
\text { - } & \text { During } \\
& \text { adolescence } \\
\text { - } & \text { During adulthood }\end{aligned}$ & $\begin{array}{l}\text { Animals were treated with THC } \\
\text { for } 10 \text { days, with behavioural } \\
\text { testing from day } 6-10 \\
\text { - Spatial learning }\end{array}$ & $\begin{array}{l}\text { Pre-treatment with THC prevented spatial learning } \\
\text { impairment in adults, but not adolescents when } \\
\text { challenged with THC. This may be related to delayed } \\
\text { activation of tolerance mechanisms in adolescents. }\end{array}$ & $\begin{array}{l}\text { (Moore, Greenleaf et } \\
\text { al. 2010) }\end{array}$ \\
\hline $\begin{aligned} \text { THC } & \\
- & \text { During } \\
& \text { adolescence }\end{aligned}$ & $\begin{array}{l}\text { Animals were treated during } \\
\text { adolescence, then tested as } \\
\text { adults } \\
\text { - Depressive-like } \\
\text { behaviour } \\
\text { - Spatial working memory }\end{array}$ & $\begin{array}{l}\text { Depressive-like behaviour in adulthood. } \\
\text { Impairment of spatial working memory related to } \\
\text { poor strategy selection in the radial maze. }\end{array}$ & $\begin{array}{l}\text { (Rubino, Vigano et al. } \\
\text { 2008; Rubino, Realini } \\
\text { et al. 2009; Rubino, } \\
\text { Realini et al. 2009) }\end{array}$ \\
\hline $\begin{array}{l}\text { Chronic WIN 55,212-2 or } \\
\text { CP 55,940 } \\
\text { - During } \\
\text { adolescence } \\
\text { - During adulthood }\end{array}$ & $\begin{array}{l}\text { All behaviours tested as adults } \\
\text { after a washout period } \\
\text { - Anxiety } \\
\text { - Working memory }\end{array}$ & $\begin{array}{l}\text { Adult animals showed no alterations in working } \\
\text { memory or anxiety, whereas all measures were } \\
\text { impaired in adulthood following adolescent } \\
\text { exposure. }\end{array}$ & $\begin{array}{l}\text { (Schneider and Koch } \\
\text { 2003) } \\
\text { (O'Shea, Singh et al. } \\
\text { 2004) }\end{array}$ \\
\hline
\end{tabular}

Table 1: Pharmacological effects of cannabinoids in adult and adolescent animals. 


\subsubsection{Behavioural Deficits in Humans}

Harvey et al (2007) investigated the relationship between cognitive performance and cannabis use in a group of human adolescents. Lasting impairment of spatial and non-spatial working memory, spatial working memory strategy and intelligence quotient after a 12 hour abstinence period was associated with adolescent cannabis use following normalisation for other variables. These deficits represent impairment of executive function, attention and working memory (Harvey, Sellman et al. 2007). Similar effects have been observed in other studies using considerably longer abstinence periods (Pope and YurgelunTodd 1996; Hall and Solowij 1998; Giancola, Shoal et al. 2001; Fried, Watkinson et al. 2005). These processes are important for individuals to efficiently process and store information. As a result of these impairments adolescent cannabis users are likely disadvantaged in learning environments (Harvey, Sellman et al. 2007) owing to the disruption of mechanisms responsible for the organisation and integration of information (Hall and Solowij 1998).

\subsection{The Hippocampal Formation}

\subsubsection{Subfields and Neural Pathways of the Hippocampal Formation}

The formation of memory, and particularly explicit memory required for spatial tasks (in which information is consciously recalled), relies on a complex network of neural circuitry within the temporal lobe (Fig. 4). The entorhinal cortex receives and integrates sensory input, primarily received from the parahippocampal cortex and perirhinal cortex. Projections from the entorhinal cortex synapse on the dentate gyrus of the hippocampus (the so called 'perforant path'), although some innervation of other hippocampal areas from the entorhinal cortex is present (Kandel, Schwarts et al. 2000). The 'trisynaptic loop' describes the pathway between the three major regions of the hippocampus. The perforant fibre pathway 
enters the hippocampus and synapses with the granule cells in the dentate gyrus. Granule cell axons, which form the mossy fibres, extend from the cell bodies in the dentate gyrus to the CA3 region. Finally, pyramidal cells in the CA3 region extend their axons to the CA1 region, forming the Schaffer collateral pathway. The output of the hippocampus is received at the subiculum, which passes information back to cortical areas via the entorhinal, parahippocampal and perirhinal cortices. The entorhinal cortex thus plays a unique role in this circuit, being simultaneously the main input and output of the hippocampus. Consequently damage to the entorhinal cortex, for example associated with the earliest pathological changes in Alzheimers disease, generate particularly severe memory impairments (Kandel, Schwarts et al. 2000; Squire, Stark et al. 2004). The hippocampus is considered to be essential for acquisition, consolidation, storage and retrieval of spatial information (D'Hooge and De Deyn 2001). Studies indicating this are discussed in the next section. 


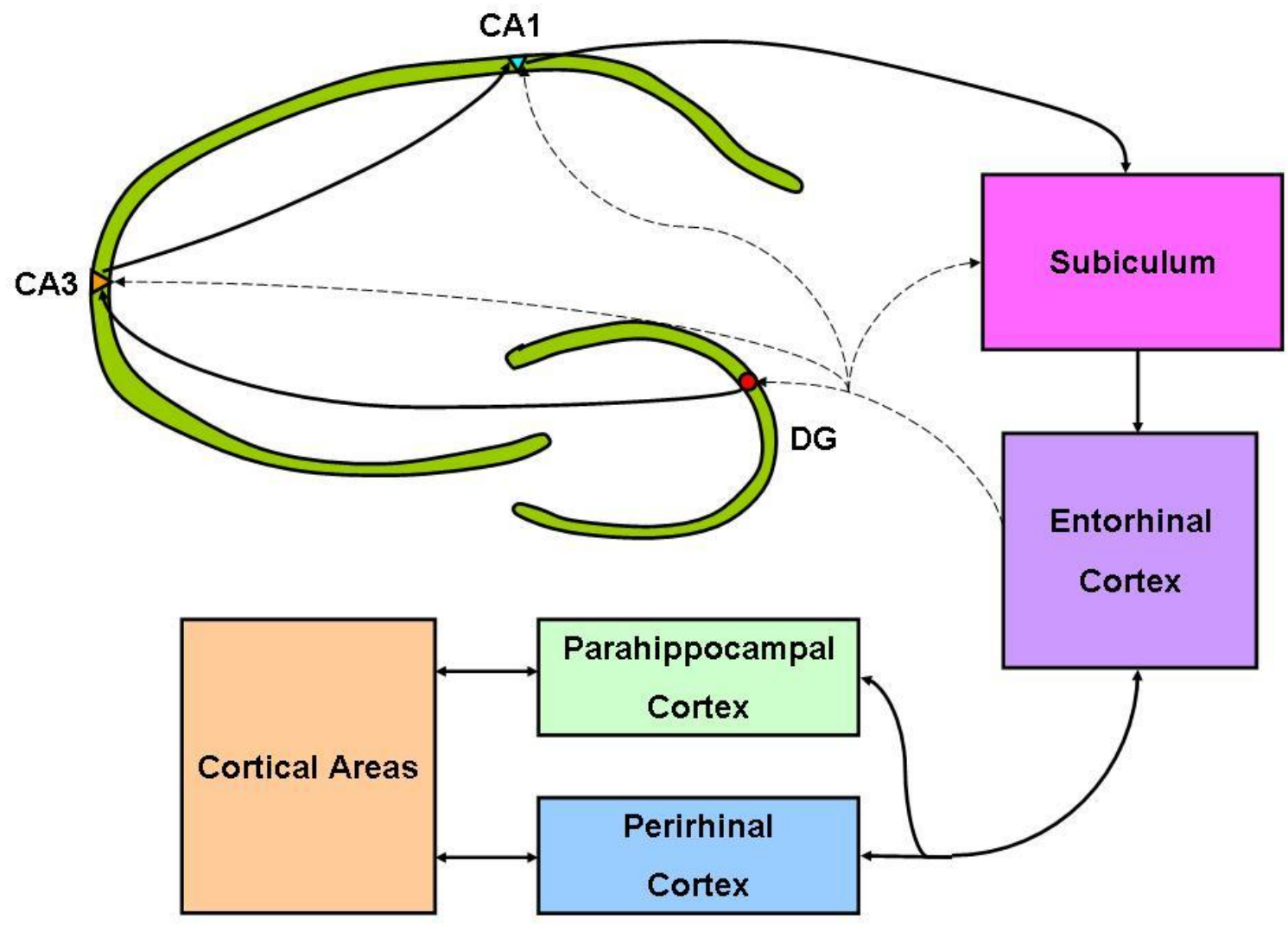

Figure 4: Neural connectivity of the temporal lobe. Sensory information from a range of cortical areas is relayed to the parahippocampal and perirhinal cortices of the temporal lobe, and this information is integrated at the level of the entorhinal cortex. This information is passed through the hippocampus sequentially, from the dentate gyrus (DG) to the CA3 and then onto the CA1 region before leaving the hippocampus through the subiculum. The entorhinal cortex primarily innervates at the level of the DG but also has inputs to other areas of the hippocampal circuit. Information is then returned to cortical areas via the associated cortices of the temporal lobe. The correct functioning of these regions, as well as the connections between them is critical in learning and memory processes. Image adapted from Kandel et al. (2000). 


\subsubsection{Importance of the Hippocampus in Learning and Memory}

The correct and coordinated functioning of several brain regions are required to perform spatial learning and memory tasks. The hippocampus is perhaps the most studied, but lesions to the striatum, basal forebrain, and several neocortical areas all produce behavioural deficits in these tasks. Lesions that disconnect the neural circuitry of these structures may be equally damaging to cognitive performance. For example, severing of the fimbra-fornix is commonly used to disrupt the neural connections between these areas. As a result of lesions to the fimbra-fornix, input from the subiculum to the nucleus accumbens, as well as cholinergic input to the hippocampus, is disrupted (D'Hooge and De Deyn 2001). The resulting hippocampal dysfunction results in spatial learning impairment (Nilsson, Shapiro et al. 1987) that is associated with behavioural inflexibility (Eichenbaum, Stewart et al. 1990).

The specific involvement of the hippocampus in spatial memory tasks such as the MWM and RAM has been demonstrate in animals with chemical lesions that induce significant cell loss in the hippocampus. Lesioned animals are able to locate the submerged platform efficiently when it is marked, thus requiring little spatial learning, although when the platform is concealed, these animals were impaired compared to lesion-free individuals (Moser, Moser et al. 1993; Pearce, Roberts et al. 1998). Furthermore, the hippocampus is especially crucial for spatial learning and memory since lesions of this type do not interfere with non-spatial learning (Gallagher and Holland 1992; Moser, Moser et al. 1993; Skinner, Martin et al. 1994; Cho, Friedman et al. 1999). However, it appears that the dorsal, rather than the ventral, hippocampus may be more important in these tasks since lesions in the dorsal area elicit a much greater impairment. This is likely due to the differential innervation of the dorsal and ventral hippocampus in which the dorsal receives inputs from the lateral entorhinal cortex and the ventral receives inputs from the medial entorhinal cortex. Sensory inputs to the dorsal hippocampus, through the lateral entorhinal cortex, include visual, auditory, somatosensory and 
olfactory processes that likely contribute significantly to spatial learning tasks (Moser, Moser et al. 1993).

More sophisticated analyses have investigated specific periods of learning and memory in spatial tasks for which intact hippocampal functioning is crucial. By infusing a water soluble AMPA receptor antagonist into the hippocampus during times of training (acquisition), memory encoding (between trials) and memory retrieval (memory), it is possible to block neurotransmission during specific phases of information processing. This method has been used to categorically illustrate that an intact hippocampus is required at all steps of spatial learning, memory and recall, as suggested by previous research (Knowlton and Fanselow 1998; Riedel, Micheau et al. 1999).

Other brain regions besides the hippocampus are important for spatial learning and memory. Lesions to the basal forebrain impair performance in spatial learning and memory tasks, presumably largely accounted for by the disruption of cholinergic output of this region that provides the hippocampus and cortex with the majority of their cholinergic inputs (Mandel, Gage et al. 1989; D'Hooge and De Deyn 2001). Lesions to subcortical regions such as the striatum also impair learning in spatial tasks (Whishaw, Mittleman et al. 1987; Block, Kunkel et al. 1993), perhaps as a consequence of impaired strategy selection (Whishaw, Mittleman et al. 1987). Finally, specific regions of the cortex are important in spatial learning and memory tasks, including the entorhinal and perirhinal cortices, as well as the prefrontal cortex. The range of cortical areas involved likely represents the range of cognitive functions required for spatial learning, in which planning and execution of required movements, as well as decision making are important (D'Hooge and De Deyn 2001). 


\subsubsection{The Cannabinoid System in the Hippocampus}

The hippocampus has amongst the highest expression levels of $C B 1 \mathrm{R}$ in the brain (Murray, Morrison et al. 2007). CB1Rs are densely expressed in several areas of the hippocampus, although in a heterogeneous manner. The pyramidal neurons of the hippocampus are covered by a dense network of CB1R (Tsou, Brown et al. 1998), a distribution pattern that is reflective of GABAergic interneurons in the hippocampus (Sloviter and Nilaver 1987). Although not seen at levels as in GABAergic neurons, CB1R is present on glutamatergic neurons in the hippocampus also (Tsou, Brown et al. 1998; Monory, Massa et al. 2006).

Endocannabinoids are known to facilitate LTP in the hippocampus by mediating subtle changes in neuronal excitability in a tightly controlled temporal and spatial manner. Briefly, endocannabinoids act as retrograde messengers that are released at postsynaptic sites and activate presynaptic CB1Rs, modulating neurotransmitter release. Endocannabinoids inhibit GABA release at low concentrations, resulting in DSI and the induction of EPSCs in the postsynaptic neuron, whereas higher concentrations of endocannabinoids inhibit glutamate release. The dual action of endocannabinoids enables the induction of LTP in a single cell amongst an unpotentiated group of neighbouring cells, while protecting against excitotoxicity (see section 1.3.2). This sensitive and specific regulation of LTP and LTD is likely to play a pivotal role in the encoding of information in the hippocampus (Chevaleyre and Castillo 2004). Exogenous application of cannabinoid such as THC could thus be expected to disrupt this fine balance between excitatory and inhibitory signals required for normal functioning, and cognitive impairment by cannabis likely involves the combined disruption of glutamatergic and GABAergic transmission. Indeed, exogenous cannabinoid agents are reported to have a serious deleterious effect on LTP (Collins, Pertwee et al. 1995; Terranova, Michaud et al. 1995; Misner and Sullivan 1999), LTD (Misner and Sullivan 1999) and a variety of cognitive processes (see sections 1.4 and 1.5). 


\subsection{The Molecular Basis of Learning and Memory}

The molecular basis of learning and memory is associated with plastic changes in the brain. The hippocampus has been the focus for much of this work as it is involved in encoding and retrieval of many types of memory, and is critical in spatial learning and memory tasks. A well characterised feature of the hippocampus is that granule cells of the dentate gyrus are continually produced beyond the embryonic period in process called neurogenesis. As newborn neurons integrate into the hippocampal circuitry, they exhibit hyperplasticity resulting in a greater probability of induction into LTP compared to mature granule cells. Furthermore, newborn neurons are rapidly growing axons, dendrites and synapses, significantly increasing the plasticity of this brain region. This effect is augmented by similar structurally plastic events in existing granule and pyramidal neurons of the hippocampus, which extend and retract their dendrites and undergo associated synapse formation and elimination. Remarkably these processes are influenced by the experiences of the individual, including learning and memory, and represent significant molecular correlates of learning and memory (reviewed in (Leuner and Gould 2010)).

\subsubsection{Senescence as a Model for Impairment in Learning and Memory Tasks}

Ageing is associated with cognitive decline in a variety of species, including humans and rats. Memory function, and especially spatial memory have been shown to be sensitive to senescence in rodents and humans. Thus, models of cognitive decline in senescence are important in understanding and identifying important neurochemical correlates of impaired memory (Bach, Barad et al. 1999; Foster 1999; Small, Tsai et al. 2002; Nyffeler, Zhang et al. 2007). 
Proper function of the medial temporal lobe is important for many forms of learning and memory, as disruption of the temporal lobe or the fibres that run between the various brain regions significantly impairs mnemonic function (see section 1.6). Functional MRI studies have shown that hippocampal dysfunction, characterised by a reduction in activity in at least one hippocampal subfield, is significantly negatively correlated with memory performance in humans (Small, Tsai et al. 2002). Interestingly, it appears that cognitive impairment associated with aging occurs in the absence of significant neuronal loss. Aged individuals with associated memory decline were found to have a comparable number of neurons in all regions of the hippocampus indicating that changes in neuronal function, rather than neuronal number, may be more important for cognitive decline (Peters, Rosene et al. 1996; Rapp and Gallagher 1996). Indeed, senescence has been associated with deficits in LTP in aged animals with associated cognitive impairment, and the maintenance of LTP appears to be especially vulnerable (Bach, Barad et al. 1999; Foster 1999). The induction and maintenance of LTP relies on a number of discrete molecules at the synaptic terminals for enhanced synaptic function and neurotransmission. It stands to reason that age related alterations in LTP will be reflected in a down-regulation of regulators of synaptic transmission.

A recent investigation of the synaptic proteome revealed that aged animals had reduced levels of molecules implicated in LTP. Impairment of exocytosis and endocytosis, as well as receptor aggregation at the synapse and synaptic maintenance in aged animals with cognitive decline could be expected from the results. In some cases these proteins appeared to be replaced by isoforms less able to perform these tasks. Impairments in these processes could be expected to result in impaired neurotransmission and replenishment of synaptic vesicle pools required to maintain LTP (VanGuilder, Yan et al. 2010). Data supporting these effects have been published. Dendritic spines represent most of the excitatory synapses in the hippocampus (Andersen 1990), and spine density is increased in LTP, likely involving the recruitment of synapses from existing and new neurons (Muller, Toni et al. 2000). Aging has also been associated with decreases in spine density and length (Nunzi, Milan et al. 1987; von Bohlen und Halbach, Zacher et 
al. 2006), as well as reductions in adult hippocampal neurogenesis (Drapeau and Abrous 2008; Leuner and Gould 2010).

Thus, neuroplasticity that encompasses the growth and branching of dendrites, reorganising of synaptic connections and neurogenesis, which allows the brain to adapt to experience, is impaired in cognitive decline associated with aging (Drapeau and Abrous 2008). The role of these processes and the involvement of specific molecules are discussed in the following sections.

\subsubsection{Hippocampal Neurogenesis}

The mammalian brain is able to produce neurons in discrete regions following birth. This is particularly evident in the dentate gyrus of the hippocampus (Fig. 5) and the subventricular zone (SVZ) of the cortex in rats (Altman and Das 1965). Neurogenesis in humans appears to be more restricted, as hippocampal neurogenesis is more modest and cortical neurogenesis is largely absent (Eriksson, Perfilieva et al. 1998; Bhardwaj, Curtis et al. 2006). Despite its comparative modesty, the preservation of hippocampal neurogenesis in humans argues for an important functional role. A body of literature that has investigated hippocampal neurogenesis in rodents suggests that these newborn neurons play a vital role in hippocampal dependent learning and memory (Bhardwaj, Curtis et al. 2006). While it has been difficult to obtain definitive evidence in humans of this important role (largely because of an inability to manipulate human hippocampal neurogenesis as we have in the rodent), the functional importance of human hippocampal neurogenesis is suggested from animal models of neurodegenerative diseases. Alzheimer's disease involves the deposit of amyloid- $\beta$ deposits in the hippocampus, a major region of neurogenesis in the human brain. In various transgenic animal models of Alzheimer's disease, deficits in neurogenesis precede the emergence of cognitive decline and memory impairment (Winner, Kohl et al. 2011). Thus although not a definitive demonstration, the overlap of Alzheimer's 
symptoms with neurogenic sites suggests a vital role of adult hippocampal neurogenesis to learning and memory in humans.

A population of astrocytic neural progenitor cells (NPCs) in the subgranular zone of the hippocampus (type 1 cells), similar in morphology to the neurogenic radial glial cells of the SVZ, has been shown to be entirely responsible for the generation of these newborn neurons in the hippocampus (Denise, Garcia et al. 2004). Division of these radial glial like cells generates transient amplifying progenitor cells (type 2 cells) that divide again to generate neuroblasts. As neuroblasts mature, they migrate into the granule cell layer (GCL), developing a process that eventually undergoes extensive branching to form the apical dendrite of mature granule cells (Seri, Manuel et al. 2004). During the first week after birth, neuroblasts migrate a short way into the GCL and extend some limited processes. In the second week, neuroblasts become polarised, extending dendrites through the GCL towards the overlying molecular layer (ML) and extending their axons through the hilus towards CA3 as they contribute to the mossy fibres. At this time, neuroblasts receive excitatory GABAergic input, and this input may be important for neuroblast survival and maturation. Excitatory GABAergic input continues until the third week when glutamatergic input is received from the perforant path, and GABA becomes inhibitory in nature. In the following weeks, newborn neurons are hyperexcitable, with a lower threshold for the induction of LTP, although these neurons are indistinguishable from mature neurons by 2 months of age (Fig. 5) (Deng, Aimone et al. 2010). Under baseline conditions, many of these neuroblasts are lost by apoptosis, although training in a hippocampal-dependent task increases the survival of these neurons (Gould, Beylin et al. 1999; Sun, Winseck et al. 2004). Neurons that successfully differentiate become mature granule cells which extend axons to the CA3 region of the hippocampus (Hastings and Gould 1999; Markakis and Gage 1999) and become functionally integrated into the trisynaptic loop (van Praag, Schinder et al. 2002; Jessberger and Kempermann 2003). 


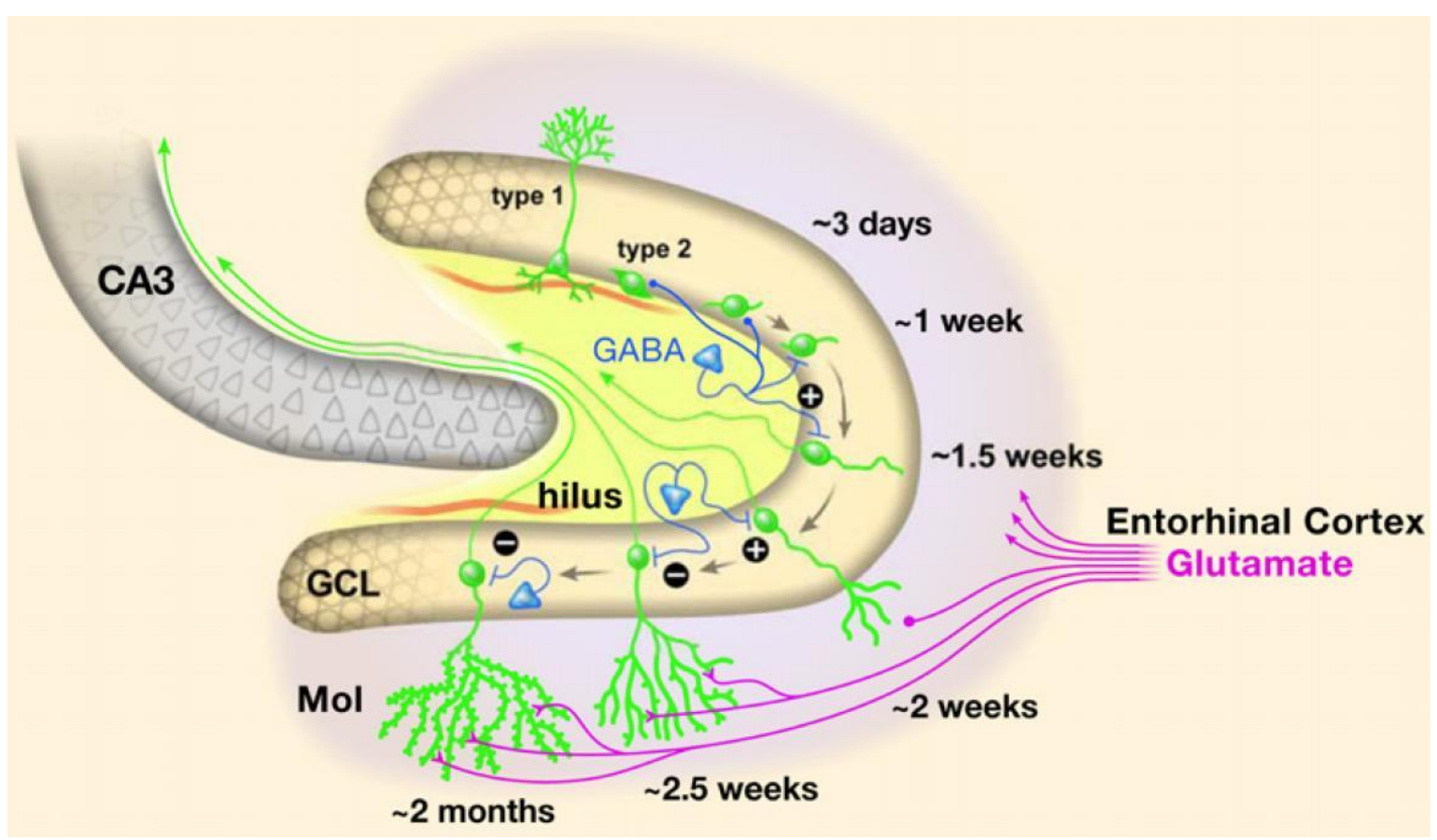

Figure 5: Development of newborn neurons in the hippocampus. Type 1 NPCs in the SGZ of the GCL divide asymmetrically generating type 2 NPCs that undergo successive rounds of division to generate a pool of young neurons. These young neurons undergo a characteristic development, migrating a short way into the GCL and extending limited processes by approximately 3 days after birth. These processes later develop into the axons and dendrites of mature neurons. By the third week of development young neurons have made some limited synapses in both the hilus and molecular layer (Mol), at which time GABAergic signalling changes from being excitatory to being inhibitory in nature. Synaptogenesis continues in the following weeks, when young neurons have a lower threshold for LTP induction and greater LTP amplitude, although these neurons are indistinguishable from mature neurons by 2 months of age. Image from Zhao et al. (2008).

There is evidence to suggest that the enhanced survival of neurons associated with performance of a learning task may not be a general survival effect. Rather, neurons of a defined age appear to be selected for, while neurons of other ages are removed. This process suggests that the selective addition and removal of neurons may be necessary based on their functional relevance (Deng, Aimone et al. 2010). Training in the MWM enhances the survival of neuroblasts born 1 week prior to training, but induces apoptosis in those born 3 days prior to training. Worse performance in the MWM was associated with lower levels of apoptosis in these 3day old neuroblasts, and this could be replicated by pharmacologically blocking 
apoptosis. Furthermore, neurons born during training were lost, although neural progenitor cell proliferation was enhanced following training in the MWM (Dupret, Fabre et al. 2007). This time period of enhanced survival coincides with the onset of GABAergic input to these neurons and the initial development of hyperexcitability, which is not present in younger neurons (Deng, Aimone et al. 2010). Furthermore, neurons that were hyperexcitable during a time of hippocampal-dependent learning (neurons from 2 weeks of age) were incorporated into circuits supporting spatial memory more frequently than mature granule cells. The selective ablation of this group of hyperexcitable neurons consistently resulted in learning and memory deficits (Clelland, Choi et al. 2009; Scobie, Hall et al. 2009), whereas other more general methods of inhibition of neurogenesis (pharmacological or irradiation methods) report inconsistent results (Deng, Aimone et al. 2010). These data suggest that newborn neurons of a defined age make a unique and important contribution to spatial memory (Kee, Teixeira et al. 2007).

Collectively, these processes may be explained on the basis of regulation of target innervation. By selecting a subset of neuroblasts to integrate into the hippocampal circuit on the basis of their functional properties, it is possible to more tightly regulate the targets these neuroblasts innervate. This likely results in a reduction of the signal to noise ratio (Dupret, Fabre et al. 2007), that would contribute significantly to what is thought to be the major function of neurogenesis in the hippocampus, a process called pattern separation (Fig. 6). A critical feature of hippocampal neurogenesis in this process is that it occurs in the informational gate to the hippocampus, the dentate gyrus. Pattern separation is used to ensure that distinct but related memories are encoded in a way that ensures interference between memories is minimised. In this way, even memories utilising similar efferent neural networks to the dentate gyrus activate very distinct afferent neural networks. Computational studies have suggested that in order to separate distinct but related memories as they are encoded, highly separated inputs are required. Neurogenesis has recently been proposed as a unique input at this level by enriching specific memories with granule cells selected on the basis of their age. Thus, neurons selected in this process function to simultaneously allow related 
memories to be recalled together, since hyperexcitability of these neurons allows them to be involved in multiple networks, whereas the afferent fibres of these neurons innervate very distinct targets preventing interference between these memories (reviewed in (Deng, Aimone et al. 2010)). Aging-related cognitive decline is associated with prolonged early development of neuroblasts, which may disrupt the fine innervation associated with neuroblasts in the hippocampus (Nyffeler, Yee et al. 2010). 


\section{a. Without Neurogenesis}
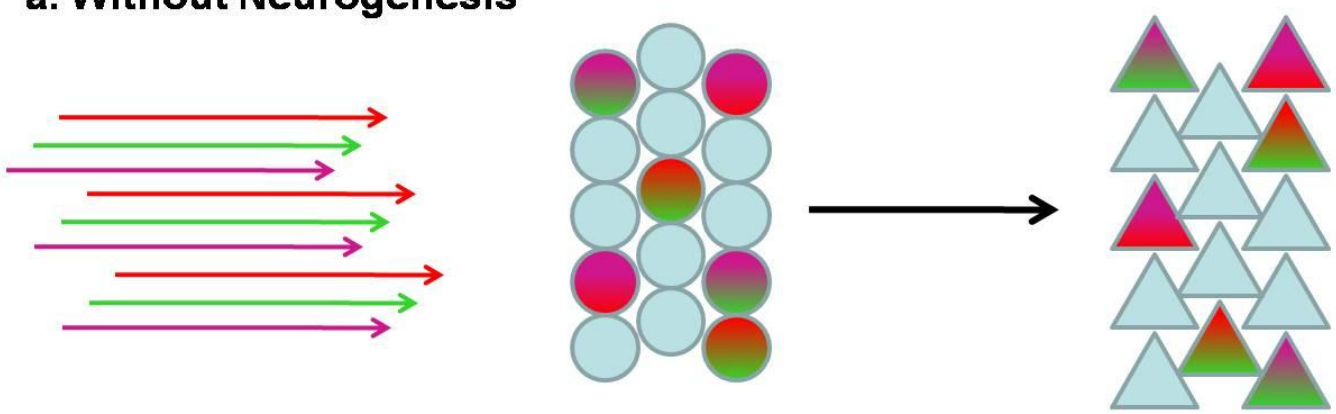

GCL

CA3

\section{b. With Neurogenesis}
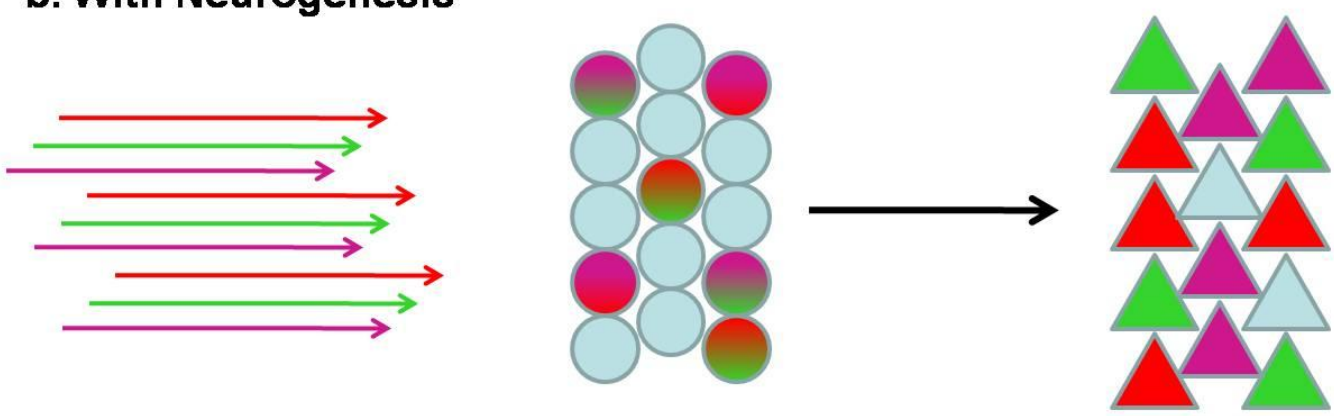

\section{Unactivated Neurons}

\section{Related informational input from the EC}

Figure 6: The role of neurogenesis in pattern separation in the hippocampus. A set of related but independent inputs to the dentate gyrus of the hippocampus from the entorhinal cortex (EC) activate a similar set of granule neurons in the granule cell layer (GCL). These activated neurons then activate neurons in the CA3 region of the hippocampus. Newborn neurons make a unique contribution to pattern separation, as their unique functional properties enable them to be incorporated into a number of distinct circuits. Without neurogenesis $(A)$ mature granule cells are unable to separate the similar input firing patterns into distinct output firing patterns. With neurogenesis (B) this same input firing pattern results in a less similar output firing pattern, reducing interference between memories while enabling related memories to be recalled together. Neurons that are activated by a specific input firing pattern are represented in the respective colour of that firing pattern, and gradients of colour within a single neuron represent shared activation by two input firing patterns. Image adapted from Deng et al. (2010). 


\subsubsection{Presynaptic Mechanisms of LTP and Synaptogenesis}

Presynaptic mechanisms of LTP largely relate to an increased likelihood of neurotransmitter release. Learning in a variety of tasks and in a range of model organisms results in an increase in the number of presynaptic inputs (Bailey and Kandel 1993; Moser 1999). A range of proteins have been implicated in the molecular cascades accounting for this phenomenon (Sanes and Lichtman 1999). For example, LTP induces the transcription of genes for synapsin-I and syntaxin 1B (Hicks, Davis et al. 1997), and learning in a spatial task increases synapsin-I protein levels (Gomez-Pinilla, So et al. 2001). Furthermore the vesicle-associated protein Rab3a is essential for LTP in mossy fibres (Castillo, Janz et al. 1997). The synapsins are especially interesting because, aside from their involvement in the induction and maintenance of LTP, they are involved in synaptic outgrowth and synaptogenesis as well as neurogenesis.

The synapsins are a group of functionally conserved yet diverse phosphoproteins (Fig. 7) associated predominantly with synaptic vesicles and account for approximately $9 \%$ of vesicle protein (Greengard, Valtorta et al. 1993). Synapsin-I, II and -III are encoded by different genes of which -I and -II may be alternatively spliced, yielding $\alpha$ and $\beta$ isoforms. Only an $\alpha$ isoform of synapsin-III has been reported (Greengard, Valtorta et al. 1993; Kao, Porton et al. 1998).

The amino-terminus of the synapsin proteins exhibit extensive homology, although there is some minor variation amongst isoforms and splice-variants. These proteins differ in their carboxy-terminals where significant variation is observed. Divergent domains link the conserved amino-terminal to one of four unique carboxy-terminal sequences (Sudhof, Czernik et al. 1989). While all synapsin proteins contain phosphorylation sites for CaMKI, CaMKIV, PKA and MAP kinase, synapsins-I and -III also share sites for CaMKII in their carboxy-terminal sequences that are not found in synapsin-II (Sudhof, Czernik et al. 1989; Kao, Porton et al. 1998). That the synapsins are substrates of so many kinases 
suggests that they may be a convergence point for multiple signalling cascades.

Furthermore, all synapsins contain actin and vesicular binding sites (Sudhof, Czernik et al. 1989), as well as a domain that binds ATP (Esser, Wang et al. 1998), although ATP binding is differentially regulated by $\mathrm{Ca}^{2+}$ concentration between synapsin isoforms (Hosaka and Sudhof 1998). Thus, the synapsins appear to be a family of highly related proteins with overlapping but divergent functions.
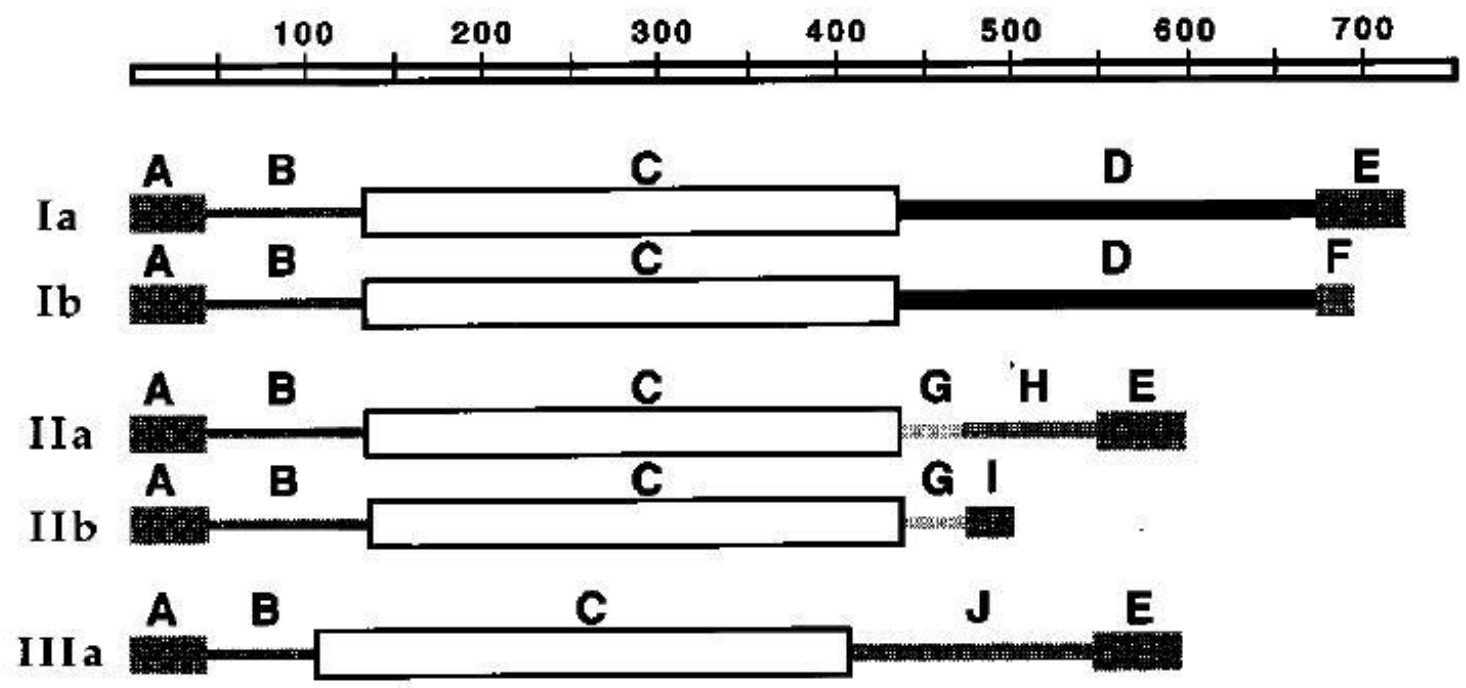

Figure 7: Domains of the synapsin proteins. Various domains are represented by the letters A $\mathrm{J}$, and shown to scale as a function of the length of their polypeptide chains (shown as amino acids, numbers at top). The amino terminal sequences of all synapsins are highly homologous (domains $\mathrm{A}-\mathrm{C}$ ), but the synapsins diverge at their carboxy-terminus. All $\alpha$-isoforms terminate in the $E$ domain, while others terminate in $F$ and I domains. The terminal regions of all synapsins are joined to the conserved $\mathrm{C}$ domain by a variable linker region (domains $\mathrm{D}, \mathrm{G}, \mathrm{H}$ and J). Image adapted from Kao et al. (1998) and Sudhof et al. (1989). 
Synapsin proteins are heavily implicated in neurotransmitter release. All synapsins are found associated with synaptic vesicles at presynaptic sites, consistent with their major role in neurotransmission in adult animals (Sudhof, Czernik et al. 1989; Feng, Chi et al. 2002; Pieribone, Porton et al. 2002). Their function at these sites is to tether synaptic vesicles and their contents to the cytoskeleton as a reserve pool, not immediately available for exocytosis upon neural stimulation (Greengard, Valtorta et al. 1993; Li, Chin et al. 1995; Pieribone, Shupliakov et al. 1995). Differences between the roles of synapsin isoforms at excitatory and inhibitory synapses have been reported, and indeed their distribution appears to differ between synapse types and brain regions (Sudhof, Czernik et al. 1989; Greengard, Valtorta et al. 1993; Feng, Chi et al. 2002). Furthermore, synapsins-I and -II, but not -III, appear to be important for the formation and maintenance of synaptic vesicles (Greengard, Valtorta et al. 1993; Rosahl, Spillane et al. 1995; Feng, Chi et al. 2002) and the increase in synaptic vesicle turnover during LTP (Li, Chin et al. 1995; Rosahl, Spillane et al. 1995). Neurotransmitter release is tightly coupled to the synapsin phosphorylation state, since synapsins are substrates for many kinases and synapsin activity is phosphorylation dependent (Rosahl, Spillane et al. 1995). For example, an increase in CaMKII activation as a result of LTP is associated with synapsin-I phosphorylation (Nayak, Moore et al. 1996) that causes a significant decrease in synapsin-I affinity for synaptic vesicles, enhancing neurotransmitter release (Navone, Digioia et al. 1989; Ceccaldi, Grohovaz et al. 1995). Interestingly, the replacement of synapsin-I, containing a CaMKII phosphorylation site, with synapsin-II, which is without this domain, is associated with age-related cognitive decline (VanGuilder, Yan et al. 2010). Additionally, in their unphosphorylated states, synapsins-I and -II are able to initiate actin bundling and therefore provide more capture sites for vesicles to prevent neurotransmitter release (Greengard, Valtorta et al. 1993).

In addition to an important role in neurotransmitter release, the synapsins play an important developmental role as well. Dissociated embryonic hippocampal neurons are a homogeneous neural population that undergoes a set of characteristic developmental changes when grown in vitro. Neurites extend from the soma before the neuron becomes polarised. Polarisation involves the 
differentiation of one of these neurites into an axon (axonogenesis) and the remaining neurites into a dendritic arbour. Axons then form synaptic connections with the arbours of neighbouring neurons (Dotti, Sullivan et al. 1988; Chin, Li et al. 1995). Knockout and knockdown studies in cell culture using this model have been used to help determine the role of the synapsin isoforms in neural development and synaptogenesis.

Synapsin-I and synapsin-II are clearly involved in neuronal differentiation and synaptogenesis in cultured hippocampal neurons, although the specific contributions of these two isotypes is unclear. Delayed axonogenesis and neurite formation have been attributed to both synapsin-I (Chin, Li et al. 1995) and synapsin-II (Ferreira, Chin et al. 1998) in two independent studies. Additionally, both synapsin isotypes have been shown to be important in synaptogenesis (Chin, Li et al. 1995; Ferreira, Chin et al. 1998), but not absolutely required since synapsin null cultures with time eventually become indistinguishable from wild type (Rosahl, Geppert et al. 1993; Ferreira, Chin et al. 1998). However, although one study has demonstrated that synapsin-II is absolutely required for the formation and maintenance of synaptic connections (Ferreira, Han et al. 1995), another has suggested that synapsin-I is more important (Rosahl, Geppert et al. 1993). Regardless of the specific contribution of each of these isotypes, it is clear that synapsins-I and -II play important roles in development and synaptogenesis. Interestingly, when these isoforms are removed simultaneously in double knockout experiments, the wild type phenotype is largely restored, demonstrating the redundancy in this system (Ferreira, Chin et al. 1998) which may contribute to the somewhat contradictory accounts of the specific roles of these synapsins in the literature.

The specific role of synapsin-III in early development is less controversial. Knockdown of synapsin-III impairs early axonal outgrowth and differentiation. However, suppression of synapsin-III after this developmental point has no effect on synaptogenesis, suggesting a specific role for synapsin-III in the establishment of neuronal polarity but not in its maintenance. Additionally, the distribution 
patterns of the synapsins are very different. Synapsin-III, but not synapsin-I or synapsin-II, is concentrated at growth cones, further suggesting a role in early development (Ferreira, Kao et al. 2000). While present at vesicles following synaptogenesis (Kao, Porton et al. 1998), synapsin-III is present at levels much lower than synapsin-I and synapsin-II (Ferreira, Kao et al. 2000). Synapsin-III mRNA levels peak early in cultured hippocampal neurons, declining during synaptogenesis, whereas synapsin-I and -II mRNA levels increase during this time period (Ferreira, Kao et al. 2000). The levels of synapsin-I and -II mRNA are known to also increase over time in postnatal animals (Zurmohle, Herms et al. 1996), and although no analyses of developmental expression have been reported, different subcellular distributions have been observed in vivo. SynapsinIII is predominantly located extrasynaptically in neurogenic regions of the adult mouse brain (Pieribone, Porton et al. 2002). The synapsin-III positive cells in these regions have been identified as migrating maturing neuroblasts rather than neural progenitor cells (Pieribone, Porton et al. 2002; Kao, Li et al. 2008). Progenitor cell proliferation was decreased, while the survival of new born neurons was increased in synapsin-III knockout animals, demonstrating a novel role for synapsin-III in proliferation, survival and differentiation of proliferative cells (Kao, Li et al. 2008). Interestingly, despite no net change in the number of surviving neurons in synapsin-III null animals (Kao, Li et al. 2008), an associated behavioural impairment in explicit memory has been described, suggesting impaired neural functioning in these animals (Porton, Rodriguiz et al. 2010). These data clearly demonstrate a significant role of synapsin-III in regulating neurogenic processes.

\subsubsection{Postsynaptic Mechanisms of LTP and Synaptogenesis}

Postsynaptic mechanisms of LTP involve alterations to the responsiveness of the postsynaptic neuron to stimulation, often via the recruitment of proteins required for neurotransmission at the synapse. A significant mechanism of LTP is an increase in the size or number of postsynaptic sites in response to learning (Bailey and Kandel 1993; Moser 1999), and this is associated with changes to the postsynaptic density (PSD). 
Dendritic spines account for nearly all excitatory post-synaptic signal transduction in neurons of vertebrates. Spines protrude from the dendrite shaft by a couple of microns and are observed extensively throughout the elaborately branched dendritic arbour. At the immediate cytosolic face of the spine, the PSD, an electron dense structure, contains the machinery required to transmit neural signals (Kennedy 2000). The chief function of the PSD is to organise a-amino-3-hydroxy5-methyl-4-isoxazolepropionate (AMPA) and $\mathrm{N}$-methyl-D-aspartate (NMDA) glutamate receptors in the postsynaptic membrane. In addition, the PSD organises signalling molecules that are required to strengthen the synapse as a result of the activation of AMPA and NMDA receptors. Each of these ionotropic receptors has different properties. Activation of AMPA receptors by binding of glutamate results in small, brief membrane depolarisations from passage of $\mathrm{Na}^{+}$and $\mathrm{K}^{+}$ions across the membrane, leading to ESPCs. Activation of NMDA receptors by glutamate allows the influx of $\mathrm{Ca}^{2+}$ ions but requires significant existing membrane depolarisation (Sanes and Lichtman 1999; Kennedy 2000). As a consequence of $\mathrm{Ca}^{2+}$ influx, several receptor systems are activated that cause increased excitability of the neuron and strengthening of the synapse by a variety of mechanisms. AMPA receptors are often involved in these effects, in which their number (Hayashi, Shi et al. 2000) or the size of current they produce upon activation (Benke, Luthi et al. 1998) is changed.

Many of the proteins required to mediate changes in plasticity resulting from $\mathrm{Ca}^{2+}$ passage through activated NMDA receptors are maintained in a highly organised structure in the PSD. The most immediate target of $\mathrm{Ca}^{2+}$ flowing through the NMDA receptor is $\mathrm{Ca}^{2+} /$ calmodulin-dependent protein kinase II (CaMKII) which is absolutely required for synaptic plasticity (Stevens, Tonegawa et al. 1994). Several $\mathrm{Ca}^{2+}$-activated signalling components are located within the PSD, including SynGAP (which when phosphorylated results in the activation of the MAPK pathway) and neuronal NOS (nNOS). The localisation of these proteins, as well as others, to the PSD relies on the scaffolding protein PSD95, which is highly co-localised with NMDA receptors at postsynaptic sites (Kennedy 2000). One of a 


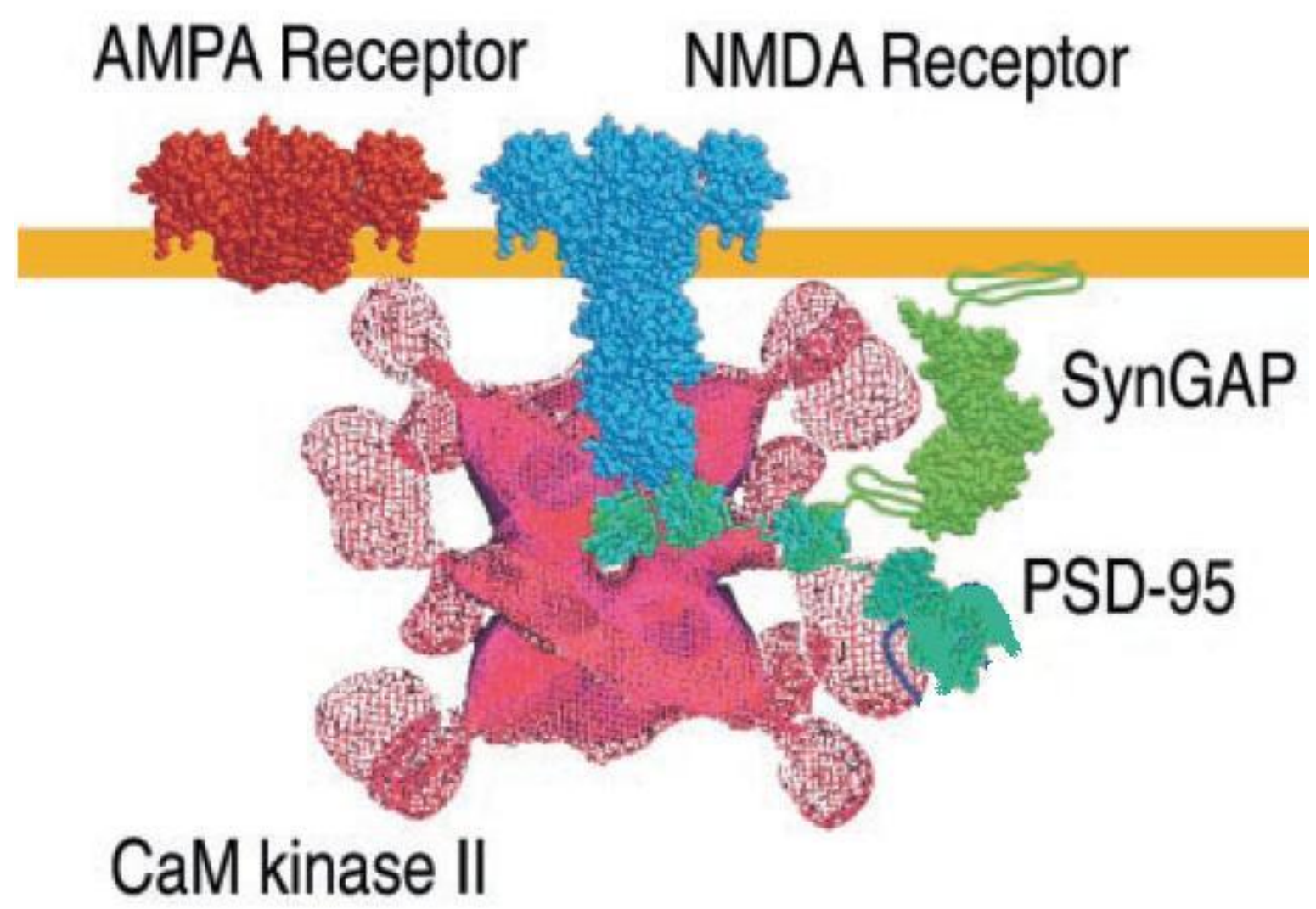

Figure 8: The molecular organisation of the PSD. The PSD95 protein organises the arrangement of the NMDA receptor signalling complex in the postsynaptic membrane. An influx of $\mathrm{Ca}^{2+}$ through the activated NMDA receptor activates CaMKII and SynGAP, amongst other $\mathrm{Ca}^{2+}$ activated signalling components required for synaptic function. The several binding domains of PSD95 organise these signal transduction molecules to the NMDA receptor, while CaMKII binds to the NMDA receptor itself. Adapted from Kennedy (2000).

family of four closely related scaffolding proteins, PSD95 contains three PDZ domains, a SH3 domain and a GuK domain (Kornau, Seeburg et al. 1997). These domains bind to the NMDA receptor, SynGAP and nNOS amongst a variety of other proteins, while CaMKII binds only to the NMDA receptor (Fig. 8). Thus, the PSD represents the core NMDA receptor signalling complex (Kennedy 2000).

Changes in the level of LTP are thought to be paralleled by morphological changes at post-synaptic sites, including in particular the appearance of perforated 
synapses (Calverley and Jones 1990; Luscher, Nicoll et al. 2000). A perforated synapse exhibits a larger, although discontinuous, PSD that is typically present in two separate and distinct areas beneath the postsynaptic membrane. Perforated synapses may then transform into duplicate synapses on separate spines, thus increasing spine density, or revert to a single synapse with an increased PSD area (Luscher, Nicoll et al. 2000; Muller, Toni et al. 2000). The occurrence of perforated synapses themselves appears to be transient, although as a result of their formation the area available for signal transduction (through the duplication of PSD in a new spine or an increased size of the PSD within a single spine) is increased (Geinisman, Detoledomorrell et al. 1991; Geinisman 1993; Muller, Toni et al. 2000). These changes in the PSD architecture appear to be mediated by AMPA receptor stimulation (Luscher, Nicoll et al. 2000; Ganeshina, Berry et al. 2004).

Using animal models of senescence-related cognitive impairment, the importance of the PSD to learning and memory processes has been demonstrated. A reduction of PSD area in perforated synapses by approximately $30 \%$ was associated with learning impairment in aged animals compared with young animals (Nicholson, Yoshida et al. 2004; Long, Liu et al. 2009). The authors, having previously shown that cognitive decline during aging is not associated with a reduction in dendritic spines, argue that perforated synapses are less efficient in this model of learning impairment (Geinisman 2000; Geinisman, Ganeshina et al. 2004). The importance of PSD95 specifically in learning and memory was demonstrated in mutant animals, as spatial learning was significantly impaired (Migaud, Charlesworth et al. 1998).

The PSD represents two functions of plasticity simultaneously. The importance of the PSD in the organisation of signalling molecules in the postsynaptic neuron is clearly related to the induction of LTP. Additionally, as these changes often involve the formation of perforated synapses, LTP increases spine density with an accompanying overall increase in PSD volume. Thus, the PSD represents plasticity at the level of both LTP and synaptogenesis. 


\subsection{The Effect of Cannabinoids on Hippocampal Plasticity}

Adult hippocampal neurogenesis and synaptogenesis are important molecular substrates of learning and memory, adding a significant degree of plasticity to the hippocampus (see section 1.7). The effect of cannabinoids on these plasticity mechanisms has been reviewed (see (Galve-Roperh, Aguado et al. 2006)), and these studies are discussed below.

\subsubsection{Cannabinoid Influences on Neurogenesis}

Both adult and embryonic neural progenitor cells contain a fully functional endocannabinoid signalling system. Enzymes required for the synthesis of 2-AG and ANA, as well as the CB1R and FAAH are observed in these cells both in vitro and in vivo (Aguado, Monory et al. 2005; Jiang, Zhang et al. 2005; Aguado, Palazuelos et al. 2006; Mulder, Aguado et al. 2008). During neurogenesis, neural progenitor cells proliferate, and their progeny differentiate and mature into neurons (see section 1.7.2). Importantly, CB1R expression is maintained on granule neurons as they mature (Wolf, Bick-Sander et al. 2010). These data strongly suggest that all stages of neurogenesis are sensitive to cannabinoid agents.

Several studies have investigated the role of cannabinoids during neurogenesis, and cannabinoids are now generally believed to stimulate neural progenitor cell proliferation. The evidence for this is that both endogenous and synthetic ligands increase proliferation in vitro and in vivo (Aguado, Monory et al. 2005; Jiang, Zhang et al. 2005; Aguado, Palazuelos et al. 2006). Similar results are observed in animals when endocannabinoid signalling is prolonged by preventing ligand degradation with $\mathrm{FAAH}^{--}$(Aguado, Monory et al. 2005; Aguado, Palazuelos et al. 2006; Mulder, Aguado et al. 2008). Conversely, abolition of cannabinoid signalling in $\mathrm{CB} 1 \mathrm{R}^{-/}$animals results in decreased neural progenitor cell proliferation (Jin, Xie et al. 2004; Aguado, Palazuelos et al. 2006; Mulder, Aguado et al. 2008). 
Cannabinoid signalling also appears to modulate the differentiation and maturation of neural progenitor-derived daughter cells. Both ANA (Rueda, Navarro et al. 2002) and the synthetic cannabinoid WIN55212,2 (Galve-Roperh, Aguado et al. 2006) delay the maturation of neurons in vitro and in vivo by a SR141716Asensitive mechanism, suggesting the involvement of CB1R. Furthermore, treatment of animals with SR141716A on its own appears to enhance neuronal maturation (Rueda, Navarro et al. 2002; Galve-Roperh, Aguado et al. 2006). Finally, it has been suggested that cannabinoids influence the differentiation decision of neural progenitor daughter cells (Aguado, Palazuelos et al. 2006). This is proposed because an increase in the astroglial phenotype of newborn cells is associated with WIN55212,2 treatment in vitro, and FAAH knockout in vivo, although endocannabinoid signalling is also described to drive the neural phenotype (Watson, Chambers et al. 2008).

Not all studies, however, agree that cannabinoids stimulate proliferation and modulate neuronal maturation. For example, proliferation in the dentate gyrus was not significantly enhanced by ANA despite other reports suggesting this (Rueda, Navarro et al. 2002). Nor did THC or WIN55212,2 have any effect on proliferation in the dentate gyrus at doses typically causing behavioural impairment (Kochman, dos Santos et al. 2006). Indeed this discontinuity was recently illustrated in a study in which THC impaired learning in the MWM in the absence of changes in cellular proliferation in the dentate gyrus (Wolf, Bick-Sander et al. 2010). This study also found no effect of THC on the survival of BrdU-labelled neurons, although survival was not assessed in animals that were trained in a spatial task and thus the effects of THC on learning-enhanced neuronal survival remain unknown (Deng, Aimone et al. 2010). Treatment of animals with SR141716A, expected to mimic the decreased proliferation observed in $\mathrm{CB} 1 \mathrm{R}^{-/}$animals, paradoxically resulted in increased proliferation in the dentate gyrus (Jin, Xie et al. 2004). This effect was preserved in $\mathrm{CB} 1 \mathrm{R}^{-/-}$animals, but ablated in $\mathrm{VR} 1^{-/-}$animals, suggesting that the action of cannabinoids on related receptor systems also plays a role in regulating neurogenic processes. Finally, both endogenous and synthetic cannabinoids have been reported to have no effect on the rate of neuronal maturation (Jiang, Zhang 
et al. 2005). Other studies have shown accelerated maturation of neurons from THC, while CB1R knockout impaired maturation (Wolf, Bick-Sander et al. 2010).

A remarkable feature of adult hippocampal neurogenesis is that experience and environment can influence the proliferation of neural progenitor cells and the survival of their progeny (see section 1.7.2). While there is some debate as to the exact role of cannabinoids on proliferation and maturation, CB1R is nevertheless present on cells that are affected by activity-dependent regulation. Recent studies have investigated the role of the cannabinoid system in these phenomena. Increased proliferation in the dentate gyrus following voluntary exercise was associated with an increase in endocannabinoid signalling. This conclusion was based on the fact that ANA synthesis, as well as an increase in CB1Rs and their functional coupling to $G$ proteins, was increased in exercised animals compared to sedentary controls (Hill, Titterness et al. 2010; Wolf, Bick-Sander et al. 2010). These adaptations were blocked by the treatment of the animals with the CB1R antagonist AM251 concurrently with free access to the running wheel (Hill, Titterness et al. 2010) and was also blocked in $\mathrm{CB}^{\mathrm{R}}{ }^{-/}$animals (Wolf, BickSander et al. 2010). Stimulation of animals by housing them in an enriched environment elicits increases in neuronal survival, similar to that observed in animals trained in a learning task. Environmental enrichment increased CB1R mRNA in the hippocampus, and the increase in neuronal survival was absent in $\mathrm{CB}_{1} \mathrm{R}^{-/-}$animals (Wolf, Bick-Sander et al. 2010). These data suggest that the endocannabinoid system contributes to, and is required for, experience-induced alterations in hippocampal plasticity (Hill, Titterness et al. 2010; Wolf, Bick-Sander et al. 2010). Surprisingly, no study has yet specifically investigated whether cannabinoids alter the enhanced survival of newborn neurons in response to training in a spatial learning and memory task. 


\subsubsection{Cannabinoid Influences on Synaptogenesis}

Cannabinoids appear to function as negative modulators of synaptogenesis by a variety of mechanisms. CB1R is enriched on GABAergic growth cones in vivo, and when grown in vitro, WIN55212,2 is able to induce GABAergic growth cone repulsion, suggesting cannabinoids are negative regulators of synaptogenesis (Berghuis, Rajnicek et al. 2007). These data are supported by findings in mouse neuroblastoma N1E-115 cells. These cells contain high levels of CB1R in their growth cones, and the synthetic cannabinoid agonist HU-210 dose-dependently induced the retraction of neurites in a mechanism that was sensitive to CB1R knockdown (Zhou and Song 2001). Furthermore, CB1R ${ }^{--}$animals showed aberrant patterns of synaptogenesis (Berghuis, Rajnicek et al. 2007; Watson, Chambers et al. 2008). Unlike GABAergic neurons, glutamatergic neurons show very little $C B 1 R$ at growth cones. Rather, these pyramidal neurons express DGL, an enzyme required for the synthesis of 2-AG (see section 1.2.2), at postsynaptic dendrites. Synthesis of 2-AG at these sites may aid in steering CB1R+ growth cones (Berghuis, Rajnicek et al. 2007), thus completing a functional endocannabinoid signalling system in which synaptogenesis is modulated. The role of the cannabinoid system of pyramidal neurons in regulating synaptogenesis is not limited to guidance of GABAergic growth cones. DGL replaces CB1R at growth cones of glutamatergic neurons (Mulder, Aguado et al. 2008), with CB1Rs being located to distal areas of the developing axon (Berghuis, Rajnicek et al. 2007). Treatment of glutamatergic neurons grown in culture with ANA inhibits; whereas, the antagonist AM251 increases axonal branching. Furthermore, inhibition of DGL results in aberrant synaptogenesis in vitro and in vivo. These data implicate endocannabinoid tone in the regulation of axonal branching and postsynaptic targeting (Mulder, Aguado et al. 2008). Thus, cannabinoids are able to influence both presynaptic and postsynaptic mechanisms of synaptogenesis in a variety of neuronal types.

The impact of cannabinoids on physiological responses to neuronal activity have been investigated, although not thoroughly. Forskolin, an activator of adenylate 
cyclase, increases the number of functional boutons in embryonic hippocampal cell cultures (Kim and Thayer 2001). The addition of the cannabinoid agonists WIN55212,2, THC, or ANA to hippocampal cultures during forskolin treatment blocks the recruitment of new synapses in a SR141716A sensitive way. These results show that abnormal levels of cannabinoid agonists during times of enhanced neural activity impair adaptive responses. However, a limitation of this study is the model of neural activity used. Forskolin treatment does not necessarily represent patterns of neuronal activation observed in vivo when performing a cognitive task. Therefore, the impairment of synaptogenesis in this model merely suggests that synaptogenesis may be impaired in vivo, and further studies are required. Surprisingly, there are no reports as to whether impairment of LTP in vivo by cannabinoids is associated with morphological changes such as alterations in spine density. One study, however, has shown that a THC-elicited reduction in LTP is associated with reduced levels of AMPA and NMDA receptors, strongly associated with dendritic spines (Fan, Yang et al. 2010).

Synaptogenesis is increased when animals perform learning and memory tasks (Gomez-Pinilla, So et al. 2001). However, only two studies by Rubino et al. have investigated whether cognitive impairment by cannabinoids is associated with altered synaptogenesis. Adolescent animals were treated with $\mathrm{THC}$ and the behavioural, molecular and morphological consequences of this treatment assessed in adulthood. Consistent with previous reports (see section 1.5.3), spatial working memory was impaired by THC in the RAM. Behavioural impairment was associated with reduced markers for presynaptic (VAMP2, synaptophyson) and postsynaptic (PSD95) connections in the hippocampus (male adolescents) and prefrontal cortex (female adolescents), as well as reduced levels of efficiency in these synapses (Rubino, Realini et al. 2009; Rubino, Realini et al. 2009). Furthermore, reduced dendritic length, dendritic branching and spine density were observed in granule cells in the dentate gyrus (Rubino, Realini et al. 2009). These data suggest that the reduction in the number or efficiency of synaptic connections in these brain areas may account for the behavioural impairment associated with cannabis treatment. A limitation of these studies was that no comparison was made between trained and untrained animals. Thus, it is 
unclear whether these results reflect a deficit in adaptive changes in response to learning, or simply a general depressive effect of the drug on these parameters. Furthermore, it is unclear whether changes in structural plasticity may underlie behavioural deficits that are measured concurrently with cannabis treatment (rather than after a period of abstinence).

Interestingly, cannabinoids also appear to inhibit synapse loss. Reducing the culture medium $\mathrm{Mg}^{2+}$ concentration to $0.1 \mathrm{nM}$ (from $0.9 \mathrm{nM}$ ) results in intense neural activity that leads to eventual synapse elimination in vitro. Both THC and the synthetic cannabinoid agonist WIN55212,2 were able to prevent network activity driven synaptic loss in cultured embryonic hippocampal neurons (Kim, Waataja et al. 2008). It is tempting to speculate that during times of synaptic loss, such as the synaptic pruning that is associated with adolescence (see section 1.6.1), disruption of the balance between synaptic loss and synaptic maintenance by cannabinoids may represent a novel molecular mechanism for behavioural impairment. There are so far, however, no studies to support this hypothesis.

\subsection{Aims of the Present Study}

There were two specific aims of this research project. The first aim was to characterise the effects of adolescent THC treatment on adolescent learning and memory (that is, behavioural assessment concurrent with THC treatment). Adolescence was investigated since few studies have examined adolescents specifically. Furthermore, adolescents may be at greater risk for the cognitive deficits associated with cannabis use. This is because adolescence is associated with significant neurodevelopmental changes, most notably with reductions in synaptic density in various brain regions including the hippocampus. In addition the expression of CB1R, responsible for mediating the cognitive effects of cannabis, peaks during adolescence before declining to adult levels. These factors may make the adolescent brain more susceptible to molecular alterations from cannabis use. There are several molecular mechanisms of learning and memory, 
including adult hippocampal neurogenesis and synaptogenesis. The effect of THC on these two mechanisms of plasticity was investigated in the hippocampus of adolescent animals, because their proposed greater sensitivity to the effects of THC represents the best chance to identify molecular correlates of THC-induced cognitive impairment. 


\section{Chapter 2 General Methods}

\subsection{Animals}

The use of rats to model human adolescence has been extensively reviewed (Spear 2000). The adolescent period in rats is considered to be between P28 $\mathrm{P} 60$, and this is generally justified by the display of behaviours that are different to both child-like and adult-like behaviour. Social interaction with peers takes on greater importance during human adolescence, and this is mirrored by increased displays of social behaviour, such as play fighting, in rodents. Risk-taking behaviour is more prevalent in both human and rodent adolescents, and this may allow adolescents to explore new environments and experiences. Indeed, adolescent rats are hyperactive when placed in a novel environment when compared to adult animals, and in the wild P28 rats emerge from the relative safety of their burrows for the first time. These risk-taking behaviours may help the adolescent to transition to adulthood. Finally the onset of puberty, growth spurts, and hyperphagia are all considered hallmarks of human adolescence, and all are seen in rats of this age range.

In this study, male Sprague Dawley rats were weaned at 3 weeks of age and housed in groups of four under 12:12 h light/dark conditions (lights on at 0700). Animals were either trained in a spatial learning and memory task (see Chapter 3 for details), or maintained as untrained home cage controls between the ages of 28 and 54 days (Spear 2000). From weaning to the time of sacrifice, access to water was ad libitum while access to food was restricted to a 5-hour window per day, typically between 1200 and 1700 following behavioural assessment. At the end of the experimental protocol, animals were sacrificed by carbon dioxide asphyxiation and rapid decapitation. All experiments involving the use of animals in this research were conducted in accordance with the Victoria University of Wellington Animal Ethics Committee guidelines and approved by the Victoria University of Wellington Animal Ethics Committee. 


\subsection{Drugs}

THC (THC Pharm GmbH the Health Concept, Germany) was dissolved in ethanol to a concentration of $30 \mathrm{mg} / \mathrm{mL}$. This stock was diluted to a working concentration of $1.5 \mathrm{mg} / \mathrm{mL}$ prepared fresh daily by mixing 1:1:18 THC:Cremophor:Saline. Animals received nightly intraperitoneal injections of either $6 \mathrm{mg} / \mathrm{kg} \mathrm{THC}$ or an equivalent volume of vehicle, beginning at 27 days of age, the eve of training commencement.

This dose of $6 \mathrm{mg} / \mathrm{kg}$ THC falls within the range of commonly used doses that result in greater cognitive impairment in adolescents, in the absence of biphasic effects of the drug (Cha, White et al. 2006; Cha, Jones et al. 2007). In addition the daily dosage regimen, where treatment continued from P27 until the time of sacrifice or the completion of training, covers a time period comparable to or greater than that used in other studies that showed a greater sensitivity of adolescents to cannabinoids. Thus this dose and time course is comparable to other treatment regimens that have shown a greater sensitivity of adolescents to cannabinoids (Quinn, Matsumoto et al. 2008; Rubino, Vigano et al. 2008; Rubino, Realini et al. 2009; Rubino, Realini et al. 2009; Moore, Greenleaf et al. 2010) and is suitable to model this greater sensitivity of adolescence in the interests of identifying molecular correlates of any cognitive impairment by THC.

\subsection{5-Bromo-2-deoxyuridine (BrdU) injections}

Animals received a $100 \mathrm{mg} / \mathrm{kg}$ dose of 5-bromo-2-deoxyuridine (BrdU, SigmaAldrich, 858811) as a single intraperitoneal injection on the evening of P27. BrdU was freshly prepared in $1 \times$ PBS to a concentration of $10 \mathrm{mg} / \mathrm{mL}$. 


\subsection{Molecular Analyses}

\subsubsection{Tissue Dissection}

Animals were sacrificed the day following completion of training in a radial maze task. Whole brains were removed from the cranial cavity and cut into thick $2 \mathrm{~mm}$ serial coronal sections with razor blades using an acrylic stereotaxic brain matrixes block (Alto, AgnTho's AB, Sweden). These sections were snap-frozen with powdered dry ice and stored at $-80^{\circ} \mathrm{C}$. When required, tissue sections between interaural measurements 4 and 6 were thawed and hippocampal tissue isolated by gently peeling the hippocampus away from both the cortex and mid-brain (Fig. 9). Tissue was transferred to a $1.5 \mathrm{~mL}$ microcentrifuge tube containing $300 \mu \mathrm{L}$ TBS and homogenised using a small plastic pestle in the presence of protease (Sigma, P8340) and phosphatase (Sigma, P5726) inhibitor cocktails to slow degradation of the epitopes needed for subsequent analyses. An aliquot of hippocampal homogenate $(100 \mu \mathrm{L})$ was transferred to $400 \mu \mathrm{L}$ TRIzol LS reagent (Invitrogen, 10296-010), thoroughly mixed and immediately frozen at $-20^{\circ} \mathrm{C}$ until required for nucleic acid analysis. A volume of RIPA buffer corresponding to 4 volumes of the initial tissue mass was then added to the remaining homogenate and thoroughly mixed. This was then incubated for $10 \mathrm{~min}$ at room temperature with occasional mixing. Cellular debris and DNA were then pelleted by centrifugation at $4^{\circ} \mathrm{C}$ and the protein containing supernatant transferred to a new tube that was frozen at $20^{\circ} \mathrm{C}$ until required for analysis. 

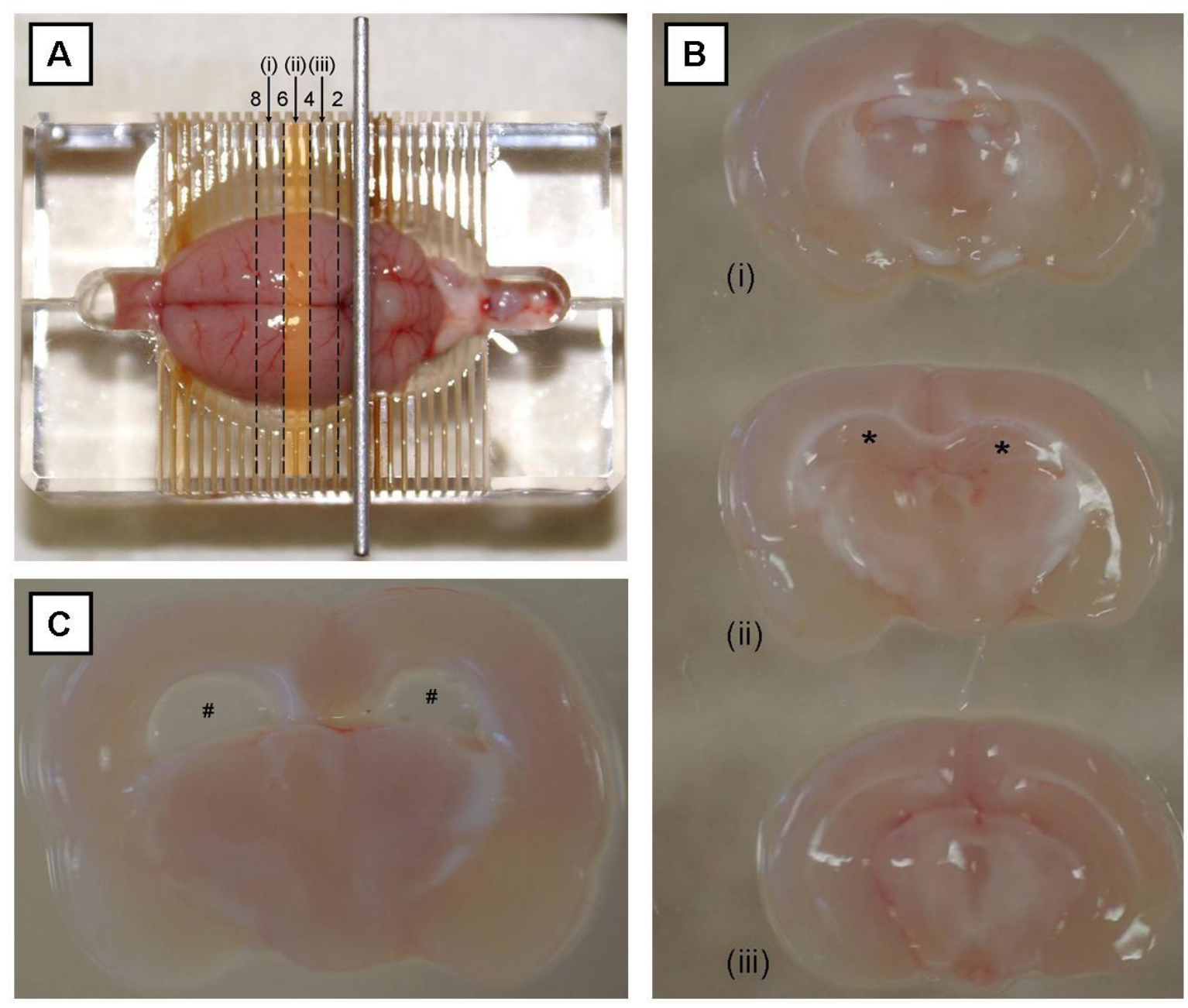

Figure 9: Dissection of the hippocampus. (A) Following removal from the cranial cavity, the intact brain was cut into $2 \mathrm{~mm}$ coronal sections beginning from interaural measurement marker zero (position shown by razor blade). The hippocampus, located between interaural positions 2 and 8 , is cut into 3 sections by this method (labelled $\mathrm{i}$, ii and iii). Tissue used for protein and RNA analysis originated from between interaural positions 4 and 6 (segment ii, highlighted). (B) The hippocampus can be observed in each of the coronal sections between interaural positions 2 and 8. The hippocampus in section ii (indicated by asterisk) was used for subsequent protein and RNA analysis. (C) Illustration of hippocampal removal from segment ii. The hippocampus has been removed (\#) while surrounding tissue remains. 


\subsubsection{Western Blotting}

\subsubsection{Protein quantification}

Total protein was quantified using a bicinchoninic acid (BCA) based assay (Thermo Scientific, 23227). In this reaction, cupric ions $\left(\mathrm{Cu}^{2+}\right)$ are reduced to cuprous ions $\left(\mathrm{Cu}^{1+}\right)$ by protein in an alkaline environment. Each cuprous ion is then chelated by two BCA molecules, and this complex exhibits strong absorbance at $562 \mathrm{~nm}$, correlating with increasing protein concentration in an almost linear relationship. Absorbance at $562 \mathrm{~nm}$ was measured using an automated multi-well plate reader (Versamax, Molecular Devices, Sunnyvale, CA). Aliquots of protein solutions were diluted to a working concentration of $1.6 \mathrm{mg} / \mathrm{mL}$ with RIPA buffer.

\subsubsection{Protein Electrophoresis and Membrane Transfer}

Protein samples were reduced with reducing buffer for $10 \mathrm{~min}$ at room temperature. Samples were not heated during this process, since the immunoreactivity of some antigens used in this study was heat sensitive. Acrylamide resolving gels were prepared fresh to a concentration of $10 \%$, overlaid with $4 \%$ stacking gels (30\% bis-acrylamide, Bio-Rad, 161-0156), and $25 \mu \mathrm{g}$ protein per lane separated by electrophoresis in $1 \mathrm{x}$ running buffer at $150 \mathrm{~V}$ for approximately $90 \mathrm{~min}$. Only one protein sample was loaded per lane. The electrophoresed protein was run concurrently with Precision Plus Protein Dual Colour size standards (Bio-Rad, 161-0374). Protein was transferred to ImmobilonFL PVDF membrane (Millipore, IPFL00010) that had been pre-soaked in methanol for $2 \mathrm{~min}$. Transfer was carried out at 20v for $17 \mathrm{~h}$ in transfer buffer. For the detection of the Ki67 epitope, $0.038 \%$ SDS was included in the transfer buffer to facilitate the transfer of high molecular weight proteins. 


\subsubsection{Antibody Incubation and Detection}

Following transfer, PVDF membranes were blocked in 1 x PBS containing 5\% BSA (Sigma, A3059) for $1 \mathrm{~h}$ at $4^{\circ} \mathrm{C}$, then incubated with the primary antibody at an appropriate dilution (Table 2) in $1 \times$ PBS containing $1 \%$ BSA for at least $4 \mathrm{~h}$ at $4^{\circ} \mathrm{C}$. The primary antibody was then washed from the PVDF membrane with two 5 -min washes in $1 \times$ TBS-T and one 5 -min wash in $1 \times$ TBS at $4^{\circ} \mathrm{C}$. The appropriate secondary antibody (Table 3), conjugated to either Alexa-488 or Alexa-555, was then incubated with the membrane for at least $2 \mathrm{~h}$ at $4^{\circ} \mathrm{C}$, then washed as described for the primary antibody. Transferred protein bound by antibody was visualised using a Fujifilm FLA5000 fluorescence scanner (Fujifilm Medical Systems, USA) at a variety of voltage settings (400-1000 V). The dataset that spanned the widest range within accurate detection limits was analysed by densitometric methods using ImageJ and normalised to the amount of protein loaded using the internal standard GAPDH. The specificity of all antibodies used in this study was confirmed by performing control experiments in which the primary antibody incubation was omitted.

\begin{tabular}{cccc}
\hline Epitope & $\begin{array}{c}\text { Host, Isotype, } \\
\text { Clonality }\end{array}$ & Manufacturer & Dilution \\
& Rabbit, IgG, polyclonal & Abcam (AB46176) & $1: 1000$ \\
BDNF & Rabbit, IgG, monoclonal & Alomone Labs (ACR-001) & $1: 2000$ \\
GAPDH & Mouse, IgG, monoclonal & Abcam (AB9484) & $1: 1000$ \\
Ki67 & Rabbit, IgG, polyclonal & Abcam (AB833) & $1: 250$ \\
PSA-NCAM & Mouse, IgM, monoclonal & Chemicon (MAB5324) & $1: 2000$ \\
PSD95 & Rabbit, IgG, polyclonal & Abcam (AB18258) & $1: 500$ \\
Sox2 & Rabbit, IgG, polyclonal & Abcam (AB97959) & $1: 1000$ \\
Synapsin 1 & Rabbit, IgG, polyclonal & Zymed (51-5200) & $1: 1000$ \\
\hline
\end{tabular}

Table 2: Primary antibodies used for Western blotting. 


\begin{tabular}{cccc}
\hline Epitope & Host & Manufacturer & Dilution \\
\hline Mouse IgG & Goat & Molecular Probes (A-11001) & $1: 1000$ \\
Mouse IgM & Goat & Molecular Probes (A-21042) & $1: 1000$ \\
Rabbit lgG & Goat & Molecular Probes (A-21429) & $1: 1000$ \\
\hline
\end{tabular}

Table 3: Secondary antibodies used in Western blotting. Antibodies were either conjugated to Alexa-488 or Alexa-555 dyes.

\subsubsection{Quantitative PCR}

\subsubsection{RNA Extraction}

Samples in TRIzol were thawed and incubated at room temperature for 5 min before centrifuging at $12000 \mathrm{xg}$ for $10 \mathrm{~min}$ at $4^{\circ} \mathrm{C}$ to remove lipids and cellular debris. The supernatant was transferred to a fresh microcentrifuge tube, and 200 $\mu \mathrm{L}$ chloroform was added for every $1 \mathrm{~mL}$ of TRIzol supernatant. Samples were vortexed thoroughly and incubated at room temperature for $3 \mathrm{~min}$, then centrifuged at $12000 \times \mathrm{g}$ for $15 \mathrm{~min}$ at $4^{\circ} \mathrm{C}$ to separate the DNA-containing organic phase from the RNA-containing aqueous phase. The aqueous phase was transferred to a fresh microcentrifuge tube, an equal volume of $70 \%$ absolute ethanol was added to precipitate the RNA and the sample thoroughly vortexed. RNA was purified using a High Pure RNA Tissue Isolation Kit (Roche, 1828 665) and eluted in 100 $\mu \mathrm{L}$ of elution buffer. RNA yield and purity were quantified with an ND-1000 Spectrophotometer (Nanodrop, Wilmington, DE) by measuring the sample absorbance at $260 \mathrm{~nm}$ and the $260 \mathrm{~nm} / 280 \mathrm{~nm}$ absorbance ratio, respectively.

\subsubsection{Reverse Transcription}

Ten $\mu \mathrm{L}$ RNA (typically containing between 250 and $450 \mu \mathrm{gNA}$ ) was reverse transcribed at $55^{\circ} \mathrm{C}$ into cDNA using Superscript III (Invitrogen, 18080-085) in the 
presence of RNAseOUT (Invitrogen, 10777-019) to inhibit RNAse A, B and C activity. Gene-specific reverse primers (2 pmol) were used to selectively reverse transcribe mRNAs to be amplified by PCR.

\subsubsection{Real-time PCR}

SYBR Green chemistry was used to quantify mRNA abundance in neural tissue. When SYBR Green binds to double stranded DNA, its fluorescent signal increases. The accumulation of PCR product with each successive round of PCR results in an increase in SYBR Green fluorescence intensity with each cycle. The amount of PCR product in each sample was determined from the cycle at which SYBR Green fluorescence rose above an arbitrary threshold (the threshold cycle, or Ct value) (Yin, Shackel et al. 2001). For each sample the Ct value for the target of interest was subtracted from the Ct value of the housekeeper gene cyclophilin $\mathrm{A}$ (to normalise for the amount of cDNA added). This value, reported as the $\Delta \mathrm{Ct}$ value, describes how abundant the transcript is in relation to the housekeeper gene. By averaging $\Delta \mathrm{Ct}$ values of different treatment groups and subtracting them from a control group a $\Delta \Delta \mathrm{Ct}$ value is obtained that describes the difference in expression levels between these two groups. A unit difference of 1 between $\Delta \mathrm{Ct}$ or $\Delta \Delta \mathrm{Ct}$ values represents a doubling (if the change is negative) or halving (if the change is positive) of transcript abundance (Livak and Schmittgen 2001).

One $\mu \mathrm{L}$ of cDNA was used per $25 \mu \mathrm{L}$ PCR reaction. PCR reactions were based on a SensiMix SYBR and Fluorescein Kit (supplied as a $2 \times$ concentrate, Bioline, QT615-05) to which template (cDNA) and primers (final concentration $200 \mathrm{nM}$ ) were added. Fluorescein allowed the iQ4 real-time PCR detection system based on an iCycler platform (Bio-Rad, Hercules, CA) to correct for variance in the baseline fluorescence of samples between reactions.

Cycling conditions were an initial denaturation and activation step at $95^{\circ} \mathrm{C}$ for 10 min, followed by 40 cycles of $95^{\circ} \mathrm{C}$ for $15 \mathrm{~s}, 55^{\circ} \mathrm{C}$ for $30 \mathrm{~s}$, then $72^{\circ} \mathrm{C}$ for $15 \mathrm{~s}$. The 
annealing temperature used for all primer sets was $55^{\circ} \mathrm{C}$ (Table 4). No template (cDNA substituted for dd $\mathrm{H} 2 \mathrm{O}$ ) and equimolar non-reverse transcribed RNA reactions were conducted for each primer pair to confirm that PCR products were specific for RNA sequences by ruling out the presence of contaminating PCR product or gDNA, respectively. Furthermore, where possible, primer pairs were designed to cross exon-exon boundaries to reduce the possibility of amplification from DNA templates.

\subsubsection{Melt-Curve Profile Analysis}

Because SYBR Green dye is unable to discriminate between specific and nonspecific PCR products, melt-curve profile analysis was carried out following the completion of 40 PCR cycles. PCR products were gradually heated from $72^{\circ} \mathrm{C}$ to $95^{\circ} \mathrm{C}$ in $0.5^{\circ} \mathrm{C}$ increments. SYBR green intercalated double stranded PCR products 'melt' into their single stranded components at a temperature that is characteristic of their specific nucleotide sequence, resulting in decreased SYBR green fluorescence. By plotting the first derivative of relative fluorescent units over temperature (i.e. rate of fluorescent change) against temperature ( $\Delta R F U / \Delta T$ vs. $T)$ a single PCR product will produce a single sharp peak. A diffuse peak between $75^{\circ} \mathrm{C}$ to $85^{\circ} \mathrm{C}$ indicates the presence of primer dimer, while multiple peaks indicate multiple PCR products (Brisson, Larissa et al. 2000).

\subsubsection{Primer Efficiency Testing}

Primer efficiencies were calculated for the primer sets used in this study. Good primer efficiency is important for the sensitivity of the reaction and primer pairs with poor efficiency will have poor sensitivity which will be especially important for low copy number transcripts (Peters, Helps et al. 2004). PCR products were purified by agarose gel electrophoresis, bands were then excised and homogenised in $600 \mu \mathrm{L} 1 \times \mathrm{TE}$ buffer before being heated at $60^{\circ} \mathrm{C}$ for $1 \mathrm{~h}$. This template solution was serially diluted over a 5 log scale and dilutions of $10^{2}-10^{5}$ 
(corresponding to $3.3 \Delta \mathrm{Ct}$ units between dilutions) were amplified by PCR in duplicate as described above. Mean Ct values were plotted against the log of the dilution to obtain the slope of the trend line. In some cases, a single dilution was excluded from the analysis if it appeared to differ significantly from the trend observed among the other data points, although at least three data points were included in all analyses. The slope of the trend line was used to determine primer efficiency using the equation:

Primer Efficiency $=\left(10^{(1 / \text { slope })}\right)-1$ (Modified from Peters et al., (2004))

Primer efficiencies of greater than $100 \%$ indicate the presence of non-specific products while efficiencies of less than $100 \%$ indicate the formation of primerdimer. The formation of either of these products can significantly reduce the amplification efficiency of the intended target, resulting in reduced data accuracy. Thus, primer efficiencies of close to $100 \%$ are desirable (Brisson, Larissa et al. 2000).

\subsubsection{Agarose Gel Electrophoresis}

PCR products were visualised with agarose gel electrophoresis to ensure the presence of a single product of the expected size. Agarose gels (2\%) were prepared by microwaving $1 \mathrm{~g}$ agarose (Invitrogen, 15510-027) in $50 \mathrm{~mL} 1 \mathrm{x}$ TAE buffer until all granules had dissolved. Ethidium bromide (Invitrogen, 15585001) was added to the molten agarose to a final concentration of $200 \mathrm{ng} / \mathrm{mL}$, and this solution was then poured into a casting tray and allowed to cool. Neat PCR product $(25 \mu \mathrm{L})$ was mixed with $5 \mu \mathrm{L}$ of $6 \times$ loading dye by repetitive pipetting, and $25 \mu \mathrm{L}$ of this solution was loaded into each lane of the agarose gel submerged in 1 x TAE buffer containing $200 \mathrm{ng} / \mathrm{mL}$ ethidium bromide. Electrophoresis was conducted at $100 \mathrm{~V}$ for $40 \mathrm{~min}$, and ethidium bromide fluorescence was visualised using a KODAK Gel Logic 100 system (Kodak). 


\begin{tabular}{|c|c|c|c|c|c|}
\hline Primer Target & \multicolumn{2}{|l|}{ Forward (+) Primer } & \multicolumn{2}{|l|}{ Reverse (-) Primer } & $\begin{array}{l}\text { Product } \\
\text { Size (bp) }\end{array}$ \\
\hline ACE & ACG GAA GCA TCA CCA AGG AG & $49^{\circ} \mathrm{C}$ & TGG CAC ATT CGC AGG AAC G & $48^{\circ} \mathrm{C}$ & 140 \\
\hline AT2R2 & CCC CTT GTT TGG TGT ATG GC & $49^{\circ} \mathrm{C}$ & AGG CAA TCC CAG CAG ACC & $47^{\circ} \mathrm{C}$ & 145 \\
\hline BDNF & CCA TAA GGA CGC GGA CTT GTA C & $52^{\circ} \mathrm{C}$ & GAG GAG GCT CCA AAG GCA CTT & $51^{\circ} \mathrm{C}$ & 68 \\
\hline Cyclophilin A & GGG GAG AAA GGA TTT GGC TA & $47^{\circ} \mathrm{C}$ & ACA TGC TTG CCA TCC ACC C & $48^{\circ} \mathrm{C}$ & 257 \\
\hline DCX & ACT GAC ATC ACA GAA GCG ATC AAA CTG G & $55^{\circ} \mathrm{C}$ & ACT GCT AGA AGT TCC ATT TGC GTC TTG G & $55^{\circ} \mathrm{C}$ & 333 \\
\hline Ki67 & CAT GGG GAT TCT GAG GCT AA & $47^{\circ} \mathrm{C}$ & GGA TCA CTG CTT GCT CTT CC & $49^{\circ} \mathrm{C}$ & 213 \\
\hline PSD95 & AGT ACC CGC TGT AGG GAT GCA GG & $56^{\circ} \mathrm{C}$ & GTG TGA AAG ACA GGG GAC CCT CAG & $56^{\circ} \mathrm{C}$ & 193 \\
\hline Synapsin-II & CAT GGG TGT TTG CTC AGA TG & $47^{\circ} \mathrm{C}$ & ACC ACG ACA GGA AAC GTA GG & $49^{\circ} \mathrm{C}$ & 127 \\
\hline Synapsin-III & CAC AGC AAG AAT GGC AGA GA & $47^{\circ} \mathrm{C}$ & TTA GTC TGT GGA CCC CAA GG & $49^{\circ} \mathrm{C}$ & 182 \\
\hline
\end{tabular}




\subsection{Immunohistochemistry}

\subsubsection{Tissue Preparation}

Following sacrifice, brains were removed from the cranial cavity and segmented as previously described (Fig. 9). Tissue was then immediately immersed in freshly prepared 4\% PFA in 1X PBS for $3 \mathrm{~h}$ with gentle agitation at room temperature then washed $3 \times 5$ min with $1 \times$ PBS. Sections were dehydrated using a graded ethanol and xylene series with two washes at each stage, changed morning and night, and then embedded in paraffin wax (McCormic Scientific, 501006). During the embedding process the concentration of paraffin wax was increased gradually to enhance the penetration of wax into the tissue, significantly improving the quality of resulting sections. This was achieved by five successive cycles of removing 0.2 volumes of xylene (in the first change) or xylene/paraffin slurry (in subsequent changes) and replacing it with molten paraffin.

\subsubsection{Tissue Sectioning}

Tissue embedded in paraffin was sectioned in the coronal plane at a thickness of $20 \mu \mathrm{m}$ using an 820 series microtome (American Optical Co., Buffalo, NY) and mounted on Superfrost Plus microscope slides (LabServ, LBS4951+).

\subsubsection{BrdU Staining of Paraffin-Embedded Sections}

Paraffin-embedded sections were deparaffinised in xylene then rehydrated though a graded ethanol series. To detect the BrdU epitope, two antigen retrieval steps were required. Sections were first boiled at $100^{\circ} \mathrm{C}$ in $10 \mathrm{mM}$ sodium citrate $\mathrm{pH} 6.0$ for $10 \mathrm{~min}$ then allowed to cool at room temperature for $30 \mathrm{~min}$; the correct $\mathrm{pH}$ of this solution was found to be vital to the success of antigen retrieval by this 
method. Next, sections were incubated with $2 \mathrm{~N} \mathrm{HCl}$ for $90 \mathrm{~min}$, then the acid was neutralised by washing sections $3 \times$ for $5 \mathrm{~min}$ in $1 \times$ PBS. Sections were then blocked in 1 x PBS containing 1\% BSA (Sigma, D-8001) for $1 \mathrm{~h}$ at room temperature, then incubated overnight at $4^{\circ} \mathrm{C}$ with an anti-BrdU mouse monoclonal antibody conjugated to biotin (1:50, Molecular Probes, B35138). The next day, sections were washed with $3 \times 5$ minute washes in $1 \times$ PBS then incubated with horseradish peroxidase (HRP) conjugated to streptavidin (1:100, Zymed, 43-4323) for $4 \mathrm{hr}$ at room temperature. Sections were processed for antigen detection by 3 , 3'-diaminobenzidine (DAB) staining.

\subsubsection{Detection by DAB Staining}

The detection of antibody bound epitopes by DAB staining relies on the localisation of the haem-containing enzyme HRP to epitope targets. HRP catalyses the oxidation of the chromogenic substrate $D A B$, in the presence of $\mathrm{H}_{2} \mathrm{O}_{2}$ and heme, to an insoluble brown product (Veitch 2004). As peroxidase is naturally present in many tissues, the endogenous peroxidase activity of samples used in this study was quenched with $3 \% \mathrm{H}_{2} \mathrm{O}_{2}$ for 30 min prior to incubation with the primary antibody to increase the signal to noise ratio. Sections were then processed as previously described. To localise HRP conjugates, sections were incubated with $1 \times$ PBS containing $0.5 \mathrm{mg} / \mathrm{mL}$ DAB (Sigma, D-8001) to which, immediately prior to incubation, $\mathrm{H}_{2} \mathrm{O}_{2}$ was added to a final concentration of $0.03 \%$. This solution was incubated on sections until sufficient colour development was observed, usually $20 \mathrm{~min}$ in this research project. Sections were rinsed in ddH2O to terminate the reaction. Sections were then counterstained with hematoxylin to stain cell nuclei for histological topography and mounted with DPX mounting medium (Fluka, 44581). Sections were examined using an Olympus AX70 photomicroscope (Olympus Optical Co., Hamburg, Germany) and images captured using an Olympus DP70 CCD camera. 


\subsection{Research Design}

The analyses presented in this study were performed on THC-treated and vehicletreated animals that both were and were not trained in the radial maze (Fig. 10). This design enabled the effects of THC on processes that occur in response to learning (by comparing trained and untrained animals for both THC-treated and vehicle-treated groups), to be separated from effects that are independent of learning (by observing the effect of THC-treatment in the absence of training). The molecular analyses were performed at a period of mid-training where behavioural deficits were expected to be slight. This was to identify molecular impairments by THC that subsequently led to more marked cognitive impairment. Behavioural testing was performed 17 hours after drug treatment. This avoided testing the cognitive effects of THC during the acute period, and rather modelled the cognitive effects likely experienced by regular patterns of cannabis use after school or in the weekends. These effects are likely more relevant to human patterns of cannabis use. 
A

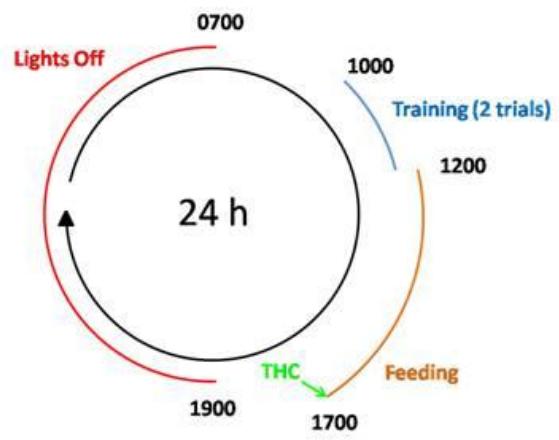

B

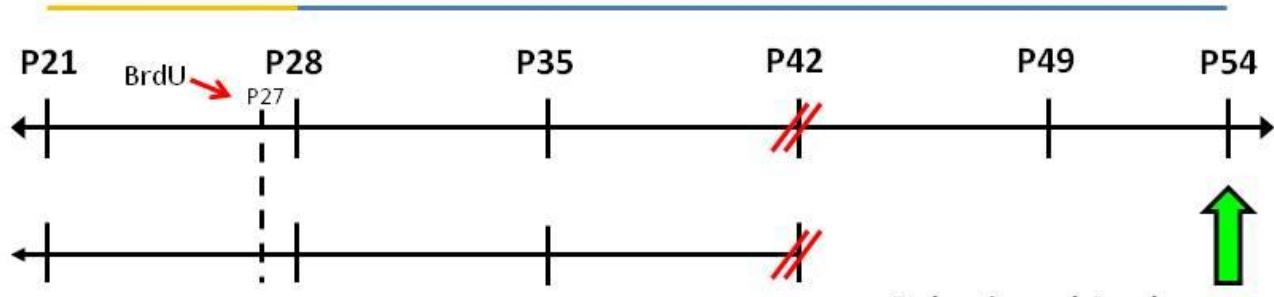

Behavioural Analyses

NO TRAINING

Molecular Analyses

Time of sacrifice

Figure 10: Training schedule and timing of treatments and analyses used in this study. A) Animals received two training trials per day, before access to food was made available for five hours. From P27 either drug- or vehicle-treatment coincided with the removal of food, 17 hours before training in the radial maze the next day. This allowed the acute effects of the drug to pass before behavioural testing, and as such the 'post-acute' effects of THC-treatment were assessed in this study. B) Behavioural analyses were performed on animals that were trained in the radial maze from P28 - P54. Animals trained in the radial maze were acclimatized to the apparatus for seven days before training began. The molecular analyses were performed on both trained and untrained groups to separate the effects of THC-treatment into the effects of treatment on processes related to training, and the effects of treatment independent of training. These analyses were performed at a time of mid-training to identify early molecular impairments leading to later cognitive impairment. All animals received a single dose of BrdU on P27 to trace the survival of newborn neurons. 


\subsection{Statistical Analysis}

All statistical analyses in this research were performed using GraphPad Prism (version 4.03 for Windows, GraphPad Software, San Diego, California, USA) and differences considered significant when $p<0.05$. Behavioural data were analysed as described in chapter 3 , while molecular data were analysed using a combination of one-way and two-way ANOVA with Bonferroni's multiple comparison test. An assumption of one-way ANOVA is that the sample groups have equal variances. To test this assumption Bartlett's test for equal variances was used. When variances differed significantly, Kruskal-Wallis with Dunn's multiple comparison test was used instead. Two-way ANOVA was used to detect effects of training (training $x$ marker) or drug treatment (treatment $x$ marker) across the range of markers of interest; whereas, one-way ANOVA was used to detect specific differences between training and drug treatment groups for a specific marker. Statistical analysis were performed using cell counts for immunohistochemistry, normalised densitometric values obtained using ImageJ for Western blots and average $\triangle \mathrm{Ct}$ values for real-time PCR. 


\section{Chapter 3 Effects of THC on Adolescent Learning}

\subsection{Introduction}

Cannabis use is commonly viewed as a culturally acceptable activity and is often initiated during adolescence (Harvey, Sellman et al. 2007; Murray, Morrison et al. 2007). Cannabis use during adolescence has a high social cost as it is associated with poorer educational achievement, as well as higher unemployment and lower life satisfaction by age 25 (Fergusson and Boden 2008).

The CB1R is predominantly responsible for mediating the effects of cannabis in the brain and is activated by the major psychoactive component of cannabis smoke, THC (Murray, Morrison et al. 2007). Spatial learning and memory tasks rely heavily on the hippocampus (Nelson, Bawa et al. 1992; Richmond, Yee et al. 1999; Ward, Stoelzel et al. 1999; Talpos, Dias et al. 2008), a brain region that expresses high levels of CB1R (Murray, Morrison et al. 2007). Implantation of the synthetic cannabinoid WIN-55,212-2 containing capillaries into the hippocampus is sufficient to impair working memory in a CB1R dependent mechanism (Barna, Soproni et al. 2007). Animal studies have shown that CB1R agonists impair, and antagonists enhance working (short-term) memory without affecting reference (factual) memory (Nakamura, Dasilva et al. 1991; Lichtman, Dimen et al. 1995; Lichtman and Martin 1996; Lichtman 2000; Mishima, Egashira et al. 2001; Wise, Iredale et al. 2008). Similar effects of cannabinoids on learning and memory have been reported in both humans and rodents (Iversen 2003).

Chunking is a strategy of organising working memory that allows discrete, individual memory units to be organised into related groups called 'chunks', which are then recalled as a whole rather than as individual entities. Memories can be continuously reorganised into fewer, but larger chunks until a suitably small 
number of large chunks is achieved to recall all the information accurately (Miller 1956). Chunking has been demonstrated in a range of species, including pigeons (Terrace 1987), rats (Dallal and Meck 1990; Cohen, Mallet et al. 1993; Macuda and Roberts 1995; Brown and Terrinoni 1996) and humans (Sakai, Kitaguchi et al. 2003; De Lillo 2004; Ridgeway 2006), suggesting that it may be an important and evolutionarily conserved process. Furthermore, chunking is considered a key mechanism of human cognition (Gobet, Lane et al. 2001). Curiously, despite the role of chunking in the organisation of working memory, and the known impairment of working memory by cannabinoids, no studies have investigated whether cannabinoid impairment of learning and memory involves an impairment of chunking.

It has been suggested that adolescents may be more susceptible to the effects of cannabis than adults (Spear 2000; Adriani and Laviola 2004); however, relatively few studies investigate the adolescent period specifically. Animals treated with cannabinoids during adolescence, but not adulthood, exhibit greater levels of anxiety and working memory impairment, as well as increased levels of depression when tested as adults (Schneider and Koch 2003; O'Shea, Singh et al. 2004; Quinn, Matsumoto et al. 2008; Rubino, Vigano et al. 2008; Rubino, Realini et al. 2009). When behavioural assessment is concurrent with drug treatment, adolescent animals generally find THC less aversive (Quinn, Matsumoto et al. 2008), although they display a greater spatial learning impairment than adult animals in the Morris water maze (Cha, White et al. 2006; Cha, Jones et al. 2007; Moore, Greenleaf et al. 2010). These data strongly suggest that developmental age plays a significant role in determining the cognitive effects of cannabis. However, these studies assessing behaviour in adulthood following adolescent treatment, or in the acute phase immediately following drug treatment, reflect long-lasting and acute impairment of the drug, respectively. Surprisingly, no studies have investigated the effect of regular patterns of use away from learning environments (e.g. after school or in the weekend) on cognitive performance between acute exposures. 
This chapter seeks to further characterise the concurrent effects of adolescent cannabis exposure on learning and memory. Specifically, testing during the acute period in which the psychoactive effects are dominant was minimised using a 'post-acute' assessment schedule instead. Furthermore, we investigated whether any deficit of learning and memory could be attributed to deficits in chunking. 'Post-acute' refers to the period of time after a THC exposure when the immediate, acute effects of THC have worn off. This assessment schedule allows a better measurement of the risks faced by adolescents who use the drug away from the learning environment (e.g. regular patterns of use after school or in the weekend), rather than the risks faced by those who are under the influence of the drug while learning.

\subsection{Methods}

\subsubsection{Animals and Drugs}

Animals were housed and fed as previously described. The study was performed twice using two separate groups of adolescent animals. Sixteen animals in each group were evenly divided into 8 control and 8 THC-treated animals in each of the two studies, resulting in a total of 16 control and 16 THC-treated animals. THC was prepared and delivered as previously described.

\subsubsection{The Radial Arm Maze}

\subsubsection{Apparatus}

An eight-arm radial maze (Olton and Samuelson 1976) was constructed with eight aluminium arms measuring 100×85×600 mm (widthxheightxlength) evenly spaced and leading from a central circular plywood platform measuring $300 \mathrm{~mm}$ in 
diameter and raised $300 \mathrm{~mm}$ from the floor of the room. The maze was painted black, and a small plastic food cup was fixed to the wall at the end of each arm. All trials were conducted in a window-less room with several distinct spatial cues present in the extra-maze environment.

\subsubsection{Acclimatisation Trials}

From weaning to day 27 , subjects were acclimatised to the radial maze. A small quantity of chocolate was deposited in each food cup, and subjects were allowed to individually explore the maze for 5 min once per day. Following the completion of the acclimatisation trial on day 27 , the eve of training, two of the four animals in each cage were randomly allocated to the THC-treatment group (total $n=8$ ) while the other two were assigned to the vehicle-treatment group (total $n=8$ ). These animals received daily intraperitoneal injections of either $6 \mathrm{mg} / \mathrm{kg} \mathrm{THC}$ or an equivalent volume of vehicle, coinciding with the removal of food at $1700 \mathrm{~h}$.

\subsubsection{Learning Trials}

From day 28 to 54, each rat completed 2 trials per day (approximately $1 \mathrm{~h}$ apart) in the reference and working memory task in the radial maze (Olton and Papas 1979). Four of the eight arms in the radial maze were baited with a small quantity of chocolate in an open dish that was not replaced during a trial. As controls, the remaining four arms were baited with the same amount of chocolate in a dish sealed but with a perforated lid to allow the scent to escape. A single baiting pattern was used throughout the study in which three of the four baited arms were adjacent to each other while the remaining baited arm was separated from the first group by one unbaited arm in the clockwise direction. The orientation of this arrangement was varied randomly between subjects by rotating the maze with regard to the spatial cues in the room. Baited arm positions were grouped into either 'chunk' $(A+B+C)$ or 'total' $(A+B+C+D)$ for analysis purposes (Fig. 11). 
Animals were kept inside an opaque cylinder measuring 200x250 mm (diameterxheight) on the central platform for $30 \mathrm{~s}$ before each trial. The cylinder was removed at the beginning of the trial, and the first four entries made by each rat were recorded. After four choices had been made or $300 \mathrm{~s}$ had elapsed, the trial was terminated, and the rat was removed from the maze. Trials were recorded using an HVS Image 2100 Plus video tracking system to facilitate analysis (HVS Image, England). An arm was considered to have been entered when the hind legs of the animal crossed a threshold one-third of the distance down the arm from the centre platform, similar to thresholds used in other studies (Lichtman 2000; Wise, Iredale et al. 2008).

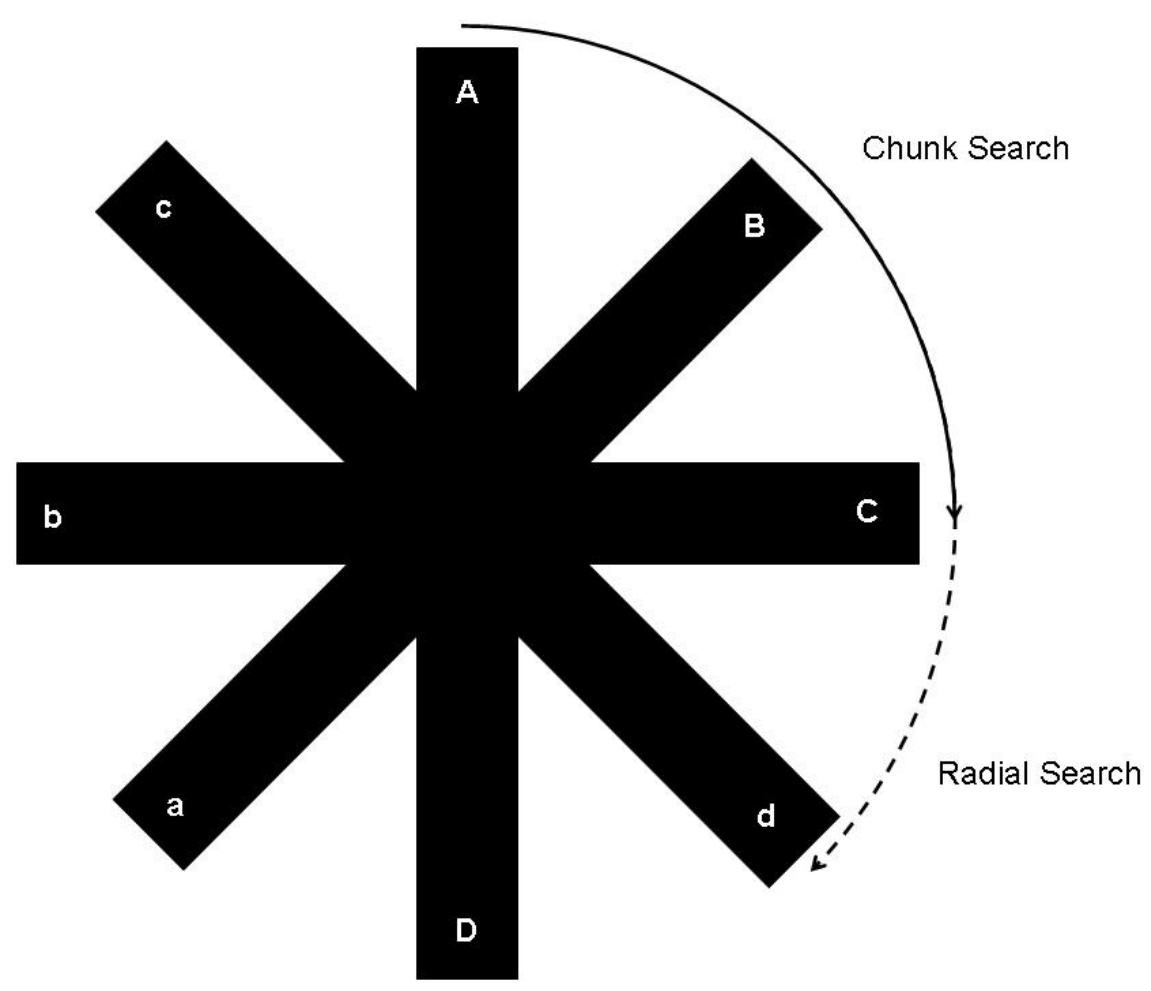

Figure 11: Configuration of the radial maze. The position of baited (uppercase letters) and unbaited (lowercase) arms in the radial maze was the same for all animals used in this study, although the spatial orientation in the room varied. Arms A, B and C constituted a 'chunk' group; whereas, all baited arms (A, B, C and D) constituted the 'total' group. 


\subsubsection{Assessment Parameters}

\subsubsection{Learning}

The arm entries made by animals in the radial maze were scored as either correct or incorrect entries based on the presence or absence of accessible chocolate in that arm. Incorrect entries were further divided into reference memory errors (entry to a non-baited arm) and working memory errors (entry to an already visited arm, regardless of whether baited or not). To be considered to have learnt the task, animals were allowed only 2 errors (any combination of working and reference memory errors) over 16 consecutive arm entries (encompassing 4 trials). For analysis purposes the 54 trials were divided into six blocks of nine trials each. The cumulative frequency of animals that had learnt the task, either in that block or in any preceding block, was expressed as a percent of all animals in their respective treatment groups (Cumulative \% achieved). Accuracy, calculated as the number of correct entries divided by the number of total entries, was used as a secondary measure of learning. The accuracy of animals in either treatment group was determined on a per block basis (36 entries in 9 trials) and as a function of overall accuracy (216 entries in 54 trials). Trial latency was measured as the time taken from the removal of the cylinder to the termination of the trial, either by the investigation of the food cup on the fourth choice or the lapsing of $300 \mathrm{~s}$. Trial latency was used to determine whether THC treatment impaired motor function (Lichtman 2000).

\subsubsection{Chunking}

The use of a single baiting pattern allowed evaluation of the strategies used by animals to solve the maze. The configuration used here specifically supported a chunking approach in which two specific and complementary behaviours were expected. The sequential collection of all rewards from the 'chunk' arm group, without visiting an arm outside this group (achievable in either three correct entries 
or four entries allowing for one working memory error) was termed 'exhaustive chunk searching' and was expected if animals chunked these arms. Because exhaustive chunk searching could be achieved by the non-spatial radial searching of four adjacent arms, the frequency of non-spatial radial searches, defined as entry into four adjacent arms in any order, was also determined. In addition, the probability that THC- and vehicle-treated animals entered each of the arms in the maze was determined to detect whether any difference in behavioural strategies enhanced the probability of entry to specific arms. Arm entry probability was calculated as the proportion of trials in which the arm was entered, calculated on an arm by arm basis (thus irrespective of what other arms were entered on the same trial).

In addition to an expected reduction in error rate as chunking associations are formed, it would be expected that entry to the spatially isolated arm would become strongly biased to either the first entry (before exhaustive chunk searching) or fourth entry (after exhaustive chunk searching). The deviation score calculation was devised to describe how biased the pattern of entry to a specific arm was, and this value was later used to measure the use of chunking strategies in the two treatment groups. The deviation score is unable to determine the exact order of preference with which an animal enters an arm. A deviation score of zero indicates that the number of entries to an arm is randomly spread over the number of visits to the arm (e.g. 20 arm visits were equally spread between entries on each of four choices allowed per trial); whereas, deviation scores other than zero indicate that the pattern was non-random. Greater deviation scores indicate a greater deviation from a random entry pattern.

To calculate the deviation score, the number of entries to each arm expected by randomness was calculated by dividing the total number of entries to the arm by four. Then, the sum of the absolute difference between the expected and actual number of entries to the arm on each of the four allowed choices was calculated. This value was then divided by the total number of entries to the arm (Equation 1). When multiple spatial locations were used to calculate a deviation score, as in the 
chunk and total baited arm patterns, the sum of the differences between observed and expected entries for all arms was divided by the sum of the total entries to all arms, rather than simply summing deviation scores for each individual arm position.

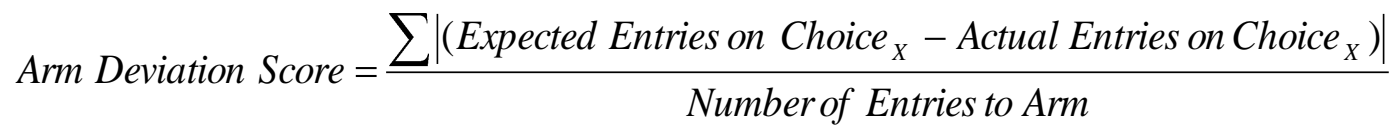

$$
\text { Expected Entries on Choice }_{X}=\frac{\text { Total Number of Entries to Arm }}{\text { Number of Choices perTrial }}
$$

\section{Equation 1: Calculation of the deviation score.}

\subsubsection{Statistical Analyses}

Linear regression was used to measure and compare the rates of learning between treatment groups. Linear regression was performed alongside the WaldWolfowitz runs test to determine wether a linear fit was appropriate for the data. Differences in either the slopes or elevations of the trend lines obtained with linear regression were interpreted as different rates of learning, as has been previously reported (Stiglick and Kalant 1982). Between-treatment group differences in trial latency, rates of reference and working memory errors, radial searches, exhaustive chunk searching, arm entry probabilities and deviation scores were assessed using two-way ANOVA with Bonferroni post-hoc tests. The number of trials required to achieve the learning criterion between treatment groups was assessed with a t-test. Within-treatment group arm entry probabilities, deviation scores, and block accuracy were assessed using one-way ANOVA with Bonferroni post-hoc tests. Correlations between chunking behaviours and either mean accuracy in the radial maze, or the block in which the learning criterion was achieved, were performed using Spearman correlation. 


\subsection{Results}

Data from the two training groups were combined since vehicle-treated animals in each group learnt the radial maze at equivalent rates. Thus, analyses are based on a pooled total of 16 THC-treated and 16 vehicle-treated animals.

\subsubsection{Maze Performance}

Trial latency was unaffected by THC-treatment, suggesting the absence of motor impairment at the dose used. Vehicle-treated animals made fewer reference memory errors than THC-treated animals $(p<0.01)$, and reference memory errors were reduced as training continued for both treatment groups ( $p<0.001$, Fig. 12A). Neither treatment, nor training affected the rate of working memory errors, and their mean occurrence was very low (Fig. 12B). THC-treated animals learnt the maze more slowly than vehicle-treated animals, seen as a difference in the slopes of the best fit lines $(p<0.05)$. THC-treated animals also required more training blocks to improve their performance over baseline (Fig. 12C), and more trials to achieve the learning criterion (Fig 12D). 

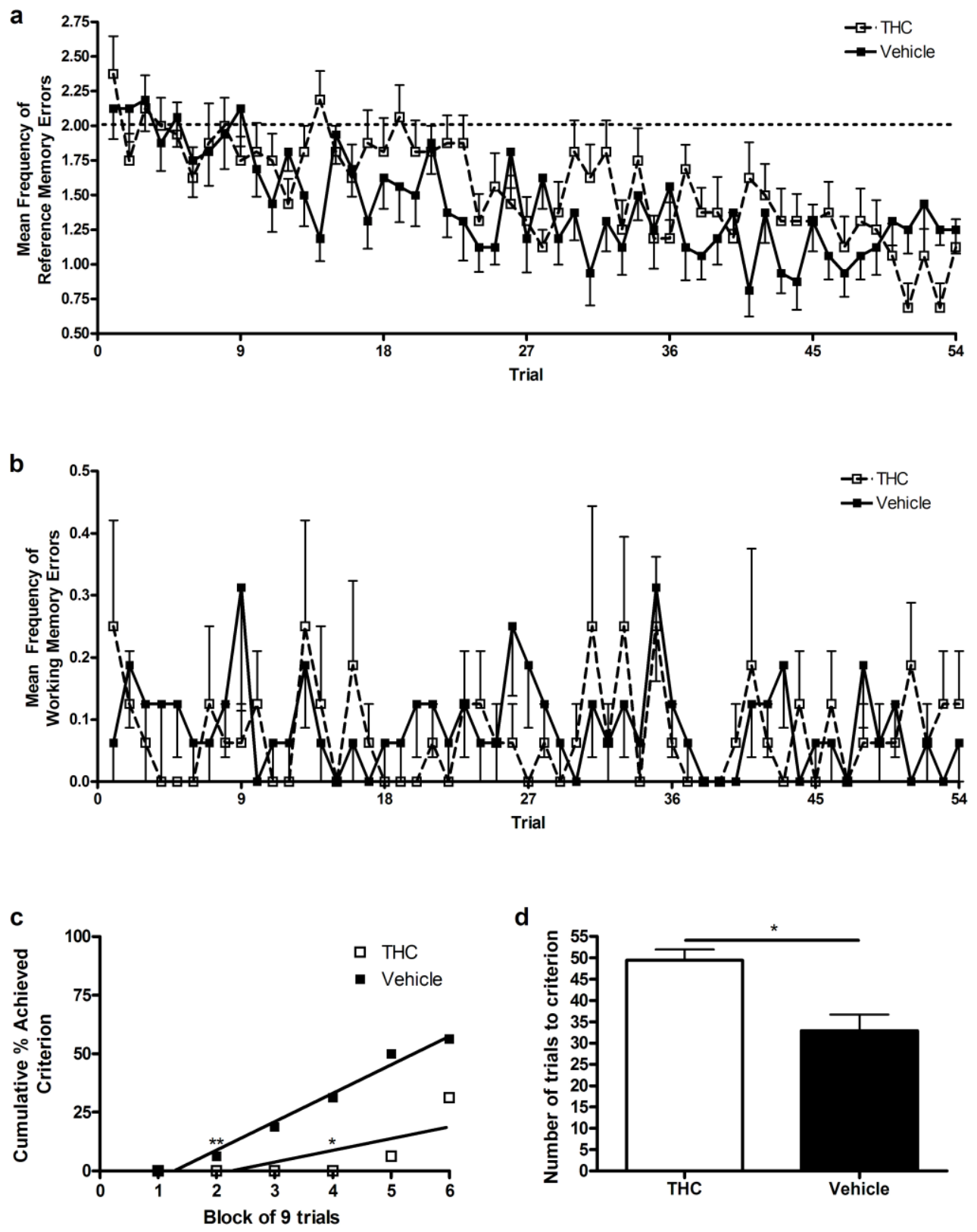

Figure 12: THC impaired learning in the radial maze. (A) THC-treated animals committed more reference memory errors than vehicle-treated animals $(p<0.01)$. Chance performance is represented by a dotted line. (B) Rates of working memory errors were not affected by THCtreatment. (C) THC-treated animals learnt the maze slower ( $p<0.05$, decreased slope of graph) and required more training to improve their accuracy over baseline levels observed in the first training block (the first point at which this was observed for each treatment group is indicated by asterisks above their respective trend lines). (D) THC-treated animals required more trials to achieve the learning criterion. Data are mean $\pm \mathrm{SEM},{ }^{*} p<0.05,{ }^{* *} p<0.01$. 


\subsubsection{Response Bias}

The utility of a chunking strategy in learning the chunking task was assessed for THC-treated and vehicle-treated animals. Exhaustive chunk searching and deviation scores at baited arm position $D$ were plotted against overall accuracy in the radial maze, as well as the block in which the learning criterion was satisfied for animals that had learnt the task. An increased incidence of both of the chunking behaviours correlated with increased accuracy and earlier learning of the task in vehicle-treated, but not THC-treated animals ( $p<0.05$, Fig. 13).To establish how the use of a chunking strategy enhanced the rate of learning in vehicletreated animals, the probability and bias of entry to each of the eight arms of the maze was compared between treatment groups.

Treatment had no significant effect on the probabilities of THC-treated and vehicletreated animals entering any specific baited arm position, although the probability of entering baited arm positions $B$ and $C$ appeared higher in vehicle-treated animals. When the data for the probability of entering individual arms of the chunk group were pooled, vehicle-treated animals made more entry to chunk arm locations than THC-treated animals ( $p<0.05$, data not shown). Both treatment groups were more likely to enter arms of the chunk than the spatially isolated arm (with the exception of baited arm position $A$ in vehicle-treated animals, that fell just outside significance). Treatment did not affect the probability of entry to unbaited arm positions, although a trend towards significance was observed $(p=0.09)$. Unbaited arm position $d$, between the chunked and isolated baited arms, was 


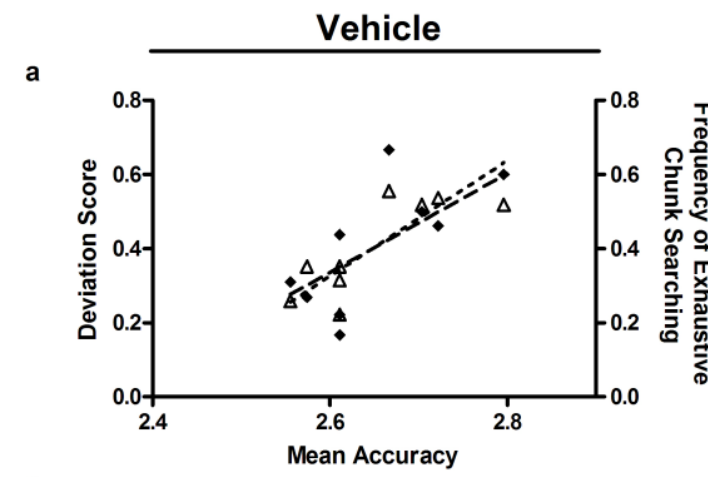

b
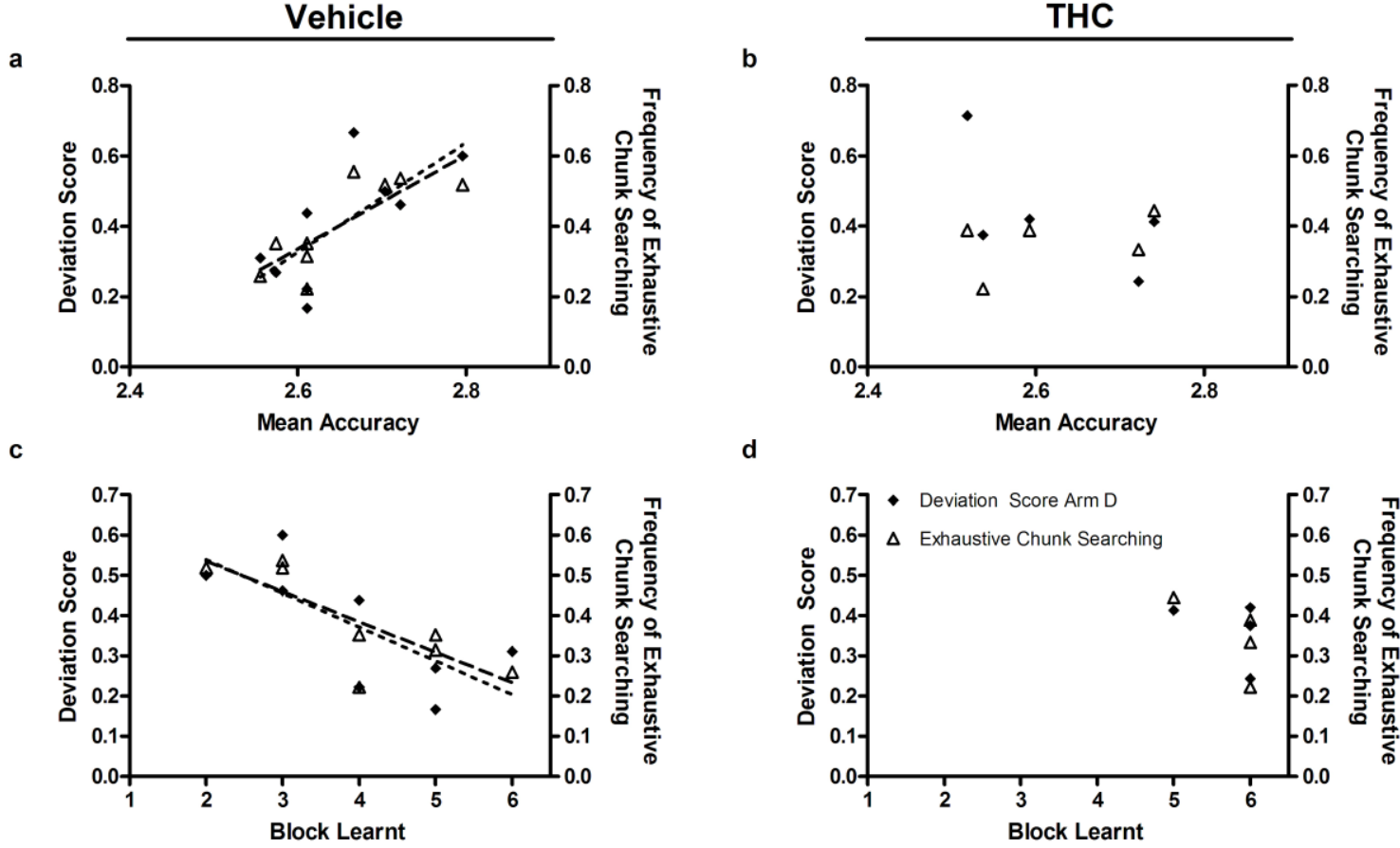

d

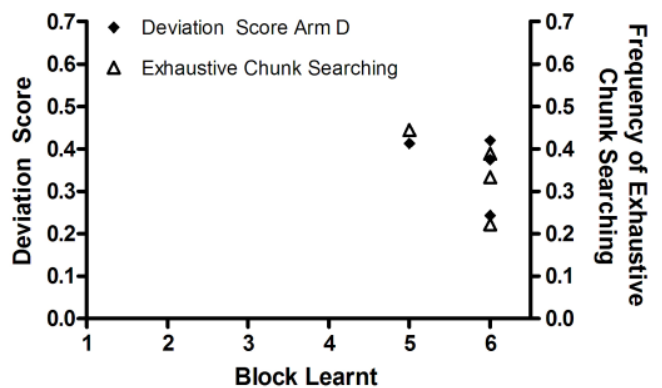

Figure 13: Chunking facilitates increased performance in vehicle-treated, but not THC-

treated animals. An increased incidence of chunking behaviours (deviation scores at arm $D$ and exhaustive chunk searching) in vehicle-treated animals was correlated with increased overall accuracy $(p<0.05, \mathrm{~A})$ and earlier learning $(p<0.05, \mathrm{C})$ in the radial maze. No correlations were observed in THC-treated animals (B, D). Trend lines: Dashed (exhaustive chunk searching), dotted (deviation scores at arm position $\mathrm{D}$ ).

equally likely to be entered by both THC-treated and vehicle-treated animals. The likelihood of entry to this arm was generally greater than any other unbaited arm position for vehicle-treated animals (with the exception of unbaited arm position a, that fell just outside significance). However, in THC-treated animals, unbaited arm position $c$, adjacent to the chunk baited group on the other side, was equally as likely to be entered as unbaited arm position $d$ (Fig. 14A). THC-treated animals had equal bias of entry to any rewarded arm or arm group as vehicle-treated animals (Fig. 14B). However, it appeared that THC-treated animals relied less on exhaustive chunk searching (Fig. 14C) and more on radial searching (Fig. 14D). The frequency of exhaustive chunk searches increased, while that of radial searching decreased with training $(p<0.001)$. 

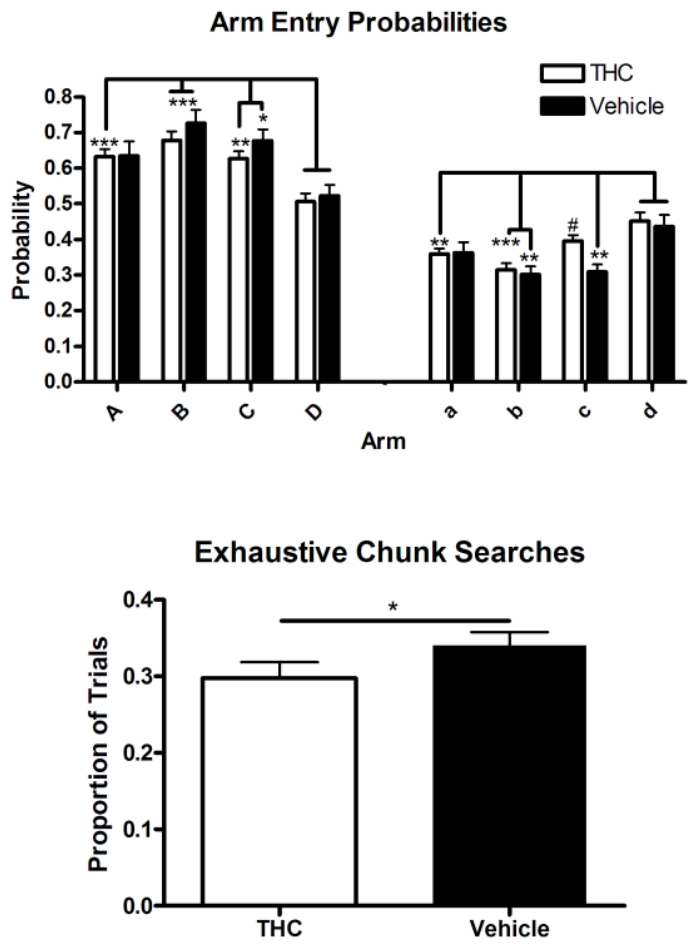

b Deviation Scores of Individual Arms or Arm Groups
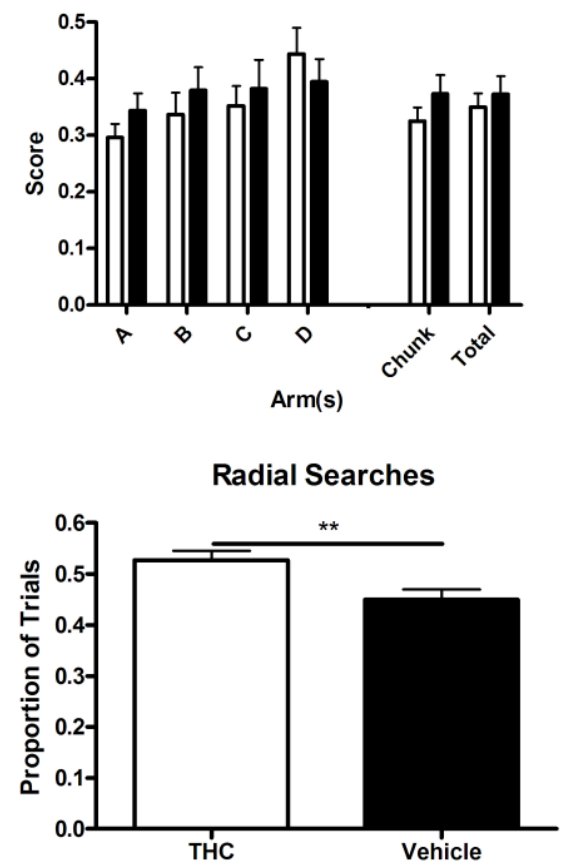

Figure 14: THC-treated animals rely less on spatial searching strategies. (A) Generally, arm $D$ (the spatially isolated, rewarded arm) was entered less frequently than arms of the chunk group (arms $A, B$ and $C$ ) for animals of both treatment groups. Animals of both treatment groups were equally likely to enter unbaited arm position $d$ (between the rewarded chunk arms and rewarded isolated arm $D$ ). Arm $d$ was generally entered more than any other unbaited arm position in vehicletreated animals. However, THC-treated animals were equally likely to enter unbaited arm position $c$, adjacent to the chunk on the opposite side as arm $d$. THC-treated animals were more likely to enter this arm than vehicle-treated animals $(p<0.01$, \#). (B) Differences in arm entry probabilities at unbaited arms did not affect levels of response bias observed for baited arm positions and arm groups between THC-treated and vehicle-treated animals. Despite equal response biases, vehicletreated animals had a higher incidence of exhaustive chunk searching (C), and THC-treated animals displayed radial search patterns in a greater proportion of trials (D). Data are mean \pm SEM; ${ }^{*} p<0.05,{ }^{* *} p<0.01,{ }^{* * *} p<0.001$. 


\subsection{Discussion}

Spatial learning and memory tasks require animals to locate rewards using environmental cues. In the radial maze, food is deposited at the end of arms radiating from a central platform. Animals improve their performance by remembering which arms contain food, which arms have already been visited, and which arm or arms they should visit next (Olton and Samuelson 1976; Olton and Papas 1979). There is some suggestion that different configurations of the same task may alter the cognitive requirements of subjects (Hodges 1996), and indeed the spatial relationship between goals influences the search strategy of rats (Dallal and Meck 1990; Brown and Terrinoni 1996; Brown and Giumetti 2006). Thus, it may be important to match the spatial configuration of goals between treatment groups, particularly if a specific cognitive process associated with any given configuration is being affected by the treatment under investigation.

These results show that THC impairs adolescent spatial learning in the radial maze task used here. THC-treated animals learnt the maze slower, required more trials to achieve the learning criterion and committed more reference memory, but not working memory errors than vehicle-treated animals. These results cannot be explained by an impairment of motivation, as food rewards were always consumed. Similarly the results cannot be explained by an impairment of motor function, as trial latencies were equivalent between treatment groups. The absence of locomotor effects is despite cannabinoids having been reported to affect motor function following acute treatment (Williams and Kirkham 2002; Shi, Luo et al. 2005; Le Foll, Wiggins et al. 2006); perhaps these effects had passed by the time of behavioural testing in this study. It is noteworthy that these results were obtained when animals were tested in the post-acute period. These data thus assess the concurrent consequences of regular patterns of cannabis use away from the learning environment (e.g. at nights or in the weekend) that affect learning when the acute effects of THC have passed (e.g. the next day at school). However, it is possible that withdrawal from THC may have contributed to these deficits. A modest withdrawal syndrome has been described in rats, with 
significant symptoms of withdrawal beginning only after 48 hours of abstinence (Aceto, Scates et al. 1996). The time scale of this study, where testing was 17 hours after drug treatment, argues against a significant withdrawal effect as results were collected more than 24 hours before these effects were described by Aceto et al. (1996).

The results presented here support previous studies that show that adolescent learning is sensitive to disruption by THC (Cha, White et al. 2006; Cha, Jones et al. 2007; Moore, Greenleaf et al. 2010). However, in these earlier studies, behavioural testing immediately followed drug treatment, thus assessing the cognitive impairment associated with the acute effects of THC. Therefore, these data appear to represent the first evidence that post-acute THC effects are able to impair adolescent learning. This is significant because post-acute effects are arguably more relevant to patterns of cannabis use commonly observed in human adolescents. The use of the radial maze, rather than the Morris water maze, is also a significant point of difference between previous studies and the results presented here, since the radial maze allows a better evaluation of specific search strategies. Recently, the radial maze was used to show that working memory impairment in adulthood, following adolescent THC treatment, is associated with the inability of THC-treated animals to utilise an appropriate search strategy (Rubino, Realini et al. 2009). However, their study was performed in the working memory paradigm of the radial maze (all arms baited), and thus does not assess any impairment of strategy selection in relation to acquisition of reference memory (learning).

Chunking describes the hierarchical recall of reference memory into working memory, facilitating improved performance over time (Miller 1956). Curiously, although spatial working memory is known to be affected by cannabinoids (Nakamura, Dasilva et al. 1991; Lichtman, Dimen et al. 1995; Lichtman 2000; Mishima, Egashira et al. 2001; Wise, Iredale et al. 2008), and chunking is known to facilitate the organisation of working memory (Miller 1956), no one has specifically investigated the effect of THC on chunking. This study used a specific 
arrangement of baited arms designed to encourage the use of a chunking strategy during learning to investigate the effects of $\mathrm{THC}$ on this strategy.

Two specific behaviours were predicted to arise from the use of a chunking strategy in this maze design; exhaustive chunk searching, and increased deviation scores at the spatially isolated arm. These behaviours were correlated with greater accuracy and earlier learning of the task in vehicle-treated animals, consistent with reports that chunking strategies facilitate earlier learning in tasks supporting a chunking approach (Dallal and Meck 1990; Macuda and Roberts 1995). However, no correlations were observed in THC-treated animals, suggesting the observed learning impairment by THC is mediated at least in part by an impairment of chunking. The benefit conferred to vehicle-treated animals by the use of a chunking strategy appeared to be an increased likelihood of entering chunk arm locations together, as indicated by a higher incidence of exhaustive chunk searching, and the fact that errors at two of these chunk arm positions were sufficient to account for the behavioural impairment in THC-treated animals. It initially seems curious that small differences in exhaustive chunk searching and arm entry probabilities result in such a robust learning deficit. However, the small magnitude of these differences is likely to be an artefact of the experimental design. Had the specific arm layout used here not supported a radial search pattern so strongly, the magnitude of these differences may have been greater.

It has been suggested that a radial search pattern may be the preferred strategy in the radial maze, except when the task prevents its efficient use (Olton, Collison et al. 1977; Dubreuil, Tixier et al. 2003; Rubino, Realini et al. 2009). The baiting arrangement used here certainly supports radial searching, as radial searches originating from all but two arms (unbaited arm positions $a$ and $b$ ) have the potential to result in the collection of three rewards. As such, a higher reliance on radial search strategies has the potential to produce similar error rates in spite of significantly different rates of learning. Indeed, THC-treated animals were equally likely to enter unbaited arm positions $c$ and $d$ that could result in the collection of three rewards from a radial search (and in addition contribute to the frequency of 
exhaustive chunk searching); whereas, vehicle-treated animals were only as likely to enter one of these locations. These data are consistent with the increased reliance of THC-treated animals on radial searching. Reliance on radial searching virtually eliminates the possibility of being considered to have learnt the task, as the learning criterion demands error rates lower than can be achieved by radial searching. Thus, despite small differences in error rates and the frequency of exhaustive chunk searching, learning was significantly impaired in THC-treated animals. It is likely that in a task that did not support radial searches so strongly, the difference in the rate of learning between THC-treated and vehicle-treated animals would be greater, as would the difference in the error rate between these two groups.

Learning in a spatial task such as the radial maze advances along a continuum, from the systematic sampling of arms relying little on spatial cues, to the formation of a cognitive map of goals as the spatial cues associated with specific goals are strengthened. Spatial goals are then chunked to reduce the difficulty of the task until accurate performance is achieved (Dallal and Meck 1990). During times of learning, chunking and the development of spatial mapping are likely parallel processes. As associations between adjacent arms are made, the information content (or size) of the chunk is gradually increased, thus reducing the cognitive load of the task and allowing more cognitive resources to be devoted to locating the remaining arms. Chunk size would continue to be gradually increased until the resources required to complete the task accurately were available (Miller 1956). Thus, by impairing chunking THC appears to slow the progression of animals along this continuum, resulting in an increased reliance on other strategies, and impairing learning as a result. As such, it is not surprising that a reduction in accuracy and a reduced reliance on chunking behaviours were observed together in THC-treated animals.

These data demonstrate for the first time that adolescent learning is sensitive to the effects of THC in the post-acute period. Significantly, they show that this reference memory impairment is due to disruption of chunking, a process of 
organising working memory. It thus appears that the attainment of reference memory (learning) is impaired by disruption of the organisation of working memory. There are, however, a number of questions arising from this study that require further investigation. For example, the relative contribution of chunking to impairment, rather than deficits in other cognitive skills, remains to be determined. Processes contributing to learning other than chunking are certainly impaired by $\mathrm{THC}$, as learning impairment by THC has been reported in the Morris water maze (Da Silva and Takahashi 2002; Cha, White et al. 2006; Cha, Jones et al. 2007; Moore, Greenleaf et al. 2010), a task that likely involves chunking to a much lesser degree than the radial maze. Titrating the relative role of the impairment of chunking by THC in radial maze tasks that support chunking to lesser degrees than the task used here would help elucidate the relative contribution of chunking to this learning impairment. Additionally the issue of developmental stage may be important, as adolescents have been shown to be more sensitive to the effects of cannabinoids than adults (Schneider and Koch 2003; O'Shea, Singh et al. 2004; Cha, White et al. 2006; Cha, Jones et al. 2007; Quinn, Matsumoto et al. 2008; Rubino, Vigano et al. 2008; Rubino, Realini et al. 2009; Moore, Greenleaf et al. 2010). As such, it will be important to determine whether chunking impairment by THC in adolescents is preserved in adults. Finally, the specific nature of the chunking impairment remains to be determined.

The chunking impairment by THC reported here operated during learning phases of a spatial task, and as such, may not be representative of the cannabinoidinduced impairment commonly associated with retrieval of already learnt information into working memory (Nakamura, Dasilva et al. 1991; Lichtman, Dimen et al. 1995; Lichtman and Martin 1996; Lichtman 2000; Mishima, Egashira et al. 2001; Wise, Iredale et al. 2008). Furthermore, these are unable to determine if the absence of chunking in THC-treated animals reflects a delay in the development of chunking, or a prevention of chunking. The assessment used in this analysis was an endpoint method, in which the involvement of chunking in learning after a defined training period was measured. It is possible that chunking in THC-treated animals may have been detected had the duration of training been extended. An impairment, rather than prevention of chunking, could explain the reduced rate of 
learning in the THC-treated group. Learning in vehicle-treated animals appeared to increase linearly, while learning in THC-treated animals was suppressed until late training when it increased rapidly. An analysis of chunking in the hierarchical radial maze would address these issues. In the hierarchical radial maze, subsets of arms are rendered more or less desirable to animals by altering the strength of reinforcers, and animals learn to retrieve more desirable rewards preferentially (Dallal and Meck 1990; Macuda and Roberts 1995; Cohen, Pardy et al. 2003). THC-treatment during the development of chunking in initial stages of training would be sufficient to assess whether THC impairs, or prevents, the formulation of chunking strategies. To assess any impairment of the use of existing chunking strategies by THC, treatment following asymptotic performance could be used. This method is also less likely to favour radial searches, and thus would aid in the measurement of the true chunking impairment operating in THC-treated animals.

The data presented in this chapter add to the few studies that have investigated the effects of THC exposure in the adolescent, rather than studying the consequences of adolescent exposure later in life. Furthermore, the data were obtained by focussing on the post-acute period in which acute drug effects were absent, since learning impairments in the post-acute period are more representative of cannabis use patterns commonly found in human adolescents. The results indicate that patterns of persistent adolescent cannabis use away from learning environments are detrimental to learning. The learning impairment elicited by THC in adolescents was mediated by impairment of chunking. Chunking organises the recall of information into working memory, facilitating earlier learning by reducing the cognitive load on working memory (Dallal and Meck 1990; Macuda and Roberts 1995). Thus the impairment of working memory, robustly affected by cannabinoids (Nakamura, Dasilva et al. 1991; Lichtman, Dimen et al. 1995; Lichtman 2000; Mishima, Egashira et al. 2001; Wise, Iredale et al. 2008), may in fact be related to an impairment of the organisation of working memory. These are the first data to suggest that chunking is affected by THC in animals of any age. Further investigation is required to fully elucidate the nature of the chunking impairment operating in THC-treated animals. For instance, whether adolescents 
are particularly sensitive to this impairment, as well as whether chunking is merely delayed or completely abolished remains to be determined. 


\section{Chapter 4 Effects of THC on Changes in Synaptic Plasticity}

\subsection{Introduction}

The molecular basis of learning and memory is largely attributed to plasticity mechanisms in the brain, and particularly in the hippocampus. Plasticity is affected by the experiences of the individual, such as during times of learning and memory (Leuner and Gould 2010). In spatial learning and memory, the hippocampus is involved in the learning of new information, the encoding of this information, and the subsequent recall of memory (Knowlton and Fanselow 1998; Riedel, Micheau et al. 1999). Thus, the hippocampus is crucial for the performance of spatial tasks. Lesions to the hippocampus (Gallagher and Holland 1992; Moser, Moser et al. 1993; Skinner, Martin et al. 1994; Pearce, Roberts et al. 1998; Cho, Friedman et al. 1999), or the fibres connecting the hippocampus to related structures (Nilsson, Shapiro et al. 1987; Eichenbaum, Stewart et al. 1990), result in cognitive impairment. Furthermore, the hippocampus expresses especially high levels of CB1R (Murray, Morrison et al. 2007), suggesting that hippocampal plasticity may be especially sensitive to impairment by cannabinoids.

Synaptic plasticity, one form of hippocampal plasticity, is critical in hippocampaldependent learning and memory. The most studied feature of synaptic plasticity in relation to learning and memory is the induction of LTP in the hippocampus. LTP describes the strengthening, or facilitation of specific synapses supporting learning in an activity dependent manner, and this form of functional plasticity is required for learning and memory processes (Martin, Grimwood et al. 2000). In addition to LTP, learning and memory is well documented to require structural changes (structural plasticity), including the recruitment of new, and reorganisation of existing synapses (Bailey and Kandel 1993; Moser 1999). Indeed the induction of LTP in the hippocampus is associated with increased synaptogenesis, with enhanced dendritic spine density (Muller, Toni et al. 2000) and transcription of 
presynaptic markers (Hicks, Davis et al. 1997). While cannabinoids are known to impair LTP in hippocampal neurons (Collins, Pertwee et al. 1995; Terranova, Michaud et al. 1995; Misner and Sullivan 1999), relatively little is known about how cannabinoids affect structural plasticity.

An impairment of synaptic recruitment by cannabinoids has been proposed as a molecular mechanism by which these drugs affect learning and memory processes. In vitro studies have shown that both synthetic and natural cannabinoids are able to prevent synaptic recruitment in response to forskolininduced neural activity (Kim and Thayer 2001). Rubino et al. have recently investigated whether behavioural impairment by THC can be attributed to changes in synaptic plasticity in vivo. In their studies, THC-treatment during adolescence appeared to impair structural and functional plasticity in adulthood, leading to cognitive impairment (Rubino, Realini et al. 2009; Rubino, Realini et al. 2009). However, the studies of Rubino et al. leave a number of important questions unanswered. As no comparison was made to untrained animals, it is unclear whether these deficits represent a particular sensitivity of synaptic plasticity to the effects of THC, or rather whether the effects of THC in the basal state culminate in cognitive impairment. Additionally, the analyses were conducted following a period of abstinence from THC, and it is unclear whether these changes in synaptic plasticity are sufficient to explain the impairment of learning and memory associated with behavioural impairment concurrent with drug treatment.

The results of Chapter 3 demonstrate that adolescent learning is sensitive to disruption by THC. Since a major molecular mechanism of learning and memory is synaptic plasticity, including structural and functional plasticity; in the present chapter, the effect of THC on synaptic plasticity was investigated. Specific markers of presynaptic and postsynaptic connections were used to evaluate synaptic plasticity in the hippocampus. The synapsins, which are involved in synaptic vesicle docking, were used as presynaptic markers; whereas, PSD95, a component of the postsynaptic density, was used as a postsynaptic marker. These proteins are tightly correlated with presynaptic (Sudhof, Czernik et al. 1989; Feng, 
Chi et al. 2002; Pieribone, Porton et al. 2002) and postsynaptic (Kennedy 2000) sites, respectively. Furthermore, despite the involvement of CB1Rs in the induction of LTP (Heifets and Castillo 2009), and the critical involvement of LTP in learning and memory processes (Martin, Grimwood et al. 2000), no one has investigated whether the expression of CB1R in the hippocampus is altered by learning of a spatial task. Thus, the effect of learning on CB1R expression was also determined.

\subsection{Methods}

\subsubsection{Animals}

Adolescent male Sprague Dawley rats were trained in the radial maze and treated daily with THC or vehicle (see section 3.2.2). The period of training was from P28 P41. This time period, representing the middle stages of training (Chapter 3 ), was selected because at this time vehicle-treated animals were beginning to learn the radial maze task, whereas $\mathrm{THC}$-treated animals were yet to significantly improve their performance (see Chapter 3). In addition to this trained group, a cohort of age and sex matched animals were identically treated with either THC or vehicle but received no training in the radial maze. Animals were maintained in their respective groups (trained or untrained) from P28 - P41, with access to food and water as described (see section 2.1), and then sacrificed on P42. All molecular analyses were conducted on hippocampal samples, collected and processed as previously described (see section 2.4).

\subsubsection{Molecular Analyses and Experimental Design}

PSD95 and the synapsins (synapsin-I, synapsin-II and synapsin-III) were used to assess the effect of adolescent THC-treatment on synaptic plasticity using Western blotting and real-time PCR. The synapsin primers used in this study were 
able to discriminate between the synapsin genes but were unable to differentiate between the $\alpha$ and $\beta$ splice variants of synapsin-I and synapsin-II. The synapsin antibody used in this research detects both the $\alpha$ and $\beta$ forms of synapsin-I, both present as a band of $\sim 80 \mathrm{kDa}$. PSD95 and CB1R are expressed as single transcripts and are present as bands of $\sim 80 \mathrm{kDa}$ and $\sim 50 \mathrm{kDa}$, respectively.

These analyses were performed on hippocampal tissue from both trained and untrained animal groups. Comparison was made between THC-treated and vehicle-treated animals trained in the radial maze, between THC-treated and vehicle-treated animals not trained in the radial maze, and between trained and untrained animals of either treatment group. Differences between THC-treated and vehicle-treated animals trained in the radial maze may represent either the sensitivity of synaptic plasticity to THC, or rather the effects of THC on the basal state resulting in cognitive impairment during testing. Differences between untrained and trained animals for either treatment group represent synaptic plasticity that occurs in response to training in the radial maze for that treatment group. Differences between untrained THC-treated and vehicle-treated animals represent effects of the drug on the basal state, not related to synaptic plasticity associated with learning, that may contribute to learning impairment.

\subsubsection{Statistical Analysis}

Measures of statistical analysis were performed as previously described (see section 2.6). Analysis of $m R N A$ expression are based on $n=8$ trained and $n=5$ untrained vehicle-treated, and $n=7$ trained and $n=5$ untrained THC-treated animals. Western blot analyses are based on $n=11$ trained and $n=10$ untrained animals for both THC-treated and vehicle-treated groups. 


\subsection{Results}

\subsubsection{Real-Time PCR Validation}

Hippocampal homogenates from THC- and vehicle-treated animals that had been either trained in the radial maze or maintained as home cage, untrained controls were analysed by real-time PCR for the expression of CB1R, PSD95, synapsin-I, synapsin-II and synapsin-III. Because the sensitivity of the real-time PCR assay can be influenced by the primer efficiency (Peters, Helps et al. 2004), and the SYBR Green dye detection system used here is unable to discriminate between intended and spurious PCR products (Brisson, Larissa et al. 2000), the efficiency and target specificity of the PCR primers were assessed.

\subsubsection{Primer Efficiencies}

Serial dilutions of purified PCR product covering a 4 log scale were amplified in duplicate, and $\mathrm{Ct}$ values plotted against the log of the dilution (Fig. 15). The slope of the trend line of these graphs was used to determine primer efficiency (Peters, Helps et al. 2004). Ideally the efficiency of primer pairs will be $100 \%$, indicating the absence of competing PCR products. This will give maximum assay sensitivity. With the exception of synapsin-I, all primer efficiencies were close to $100 \%$. 

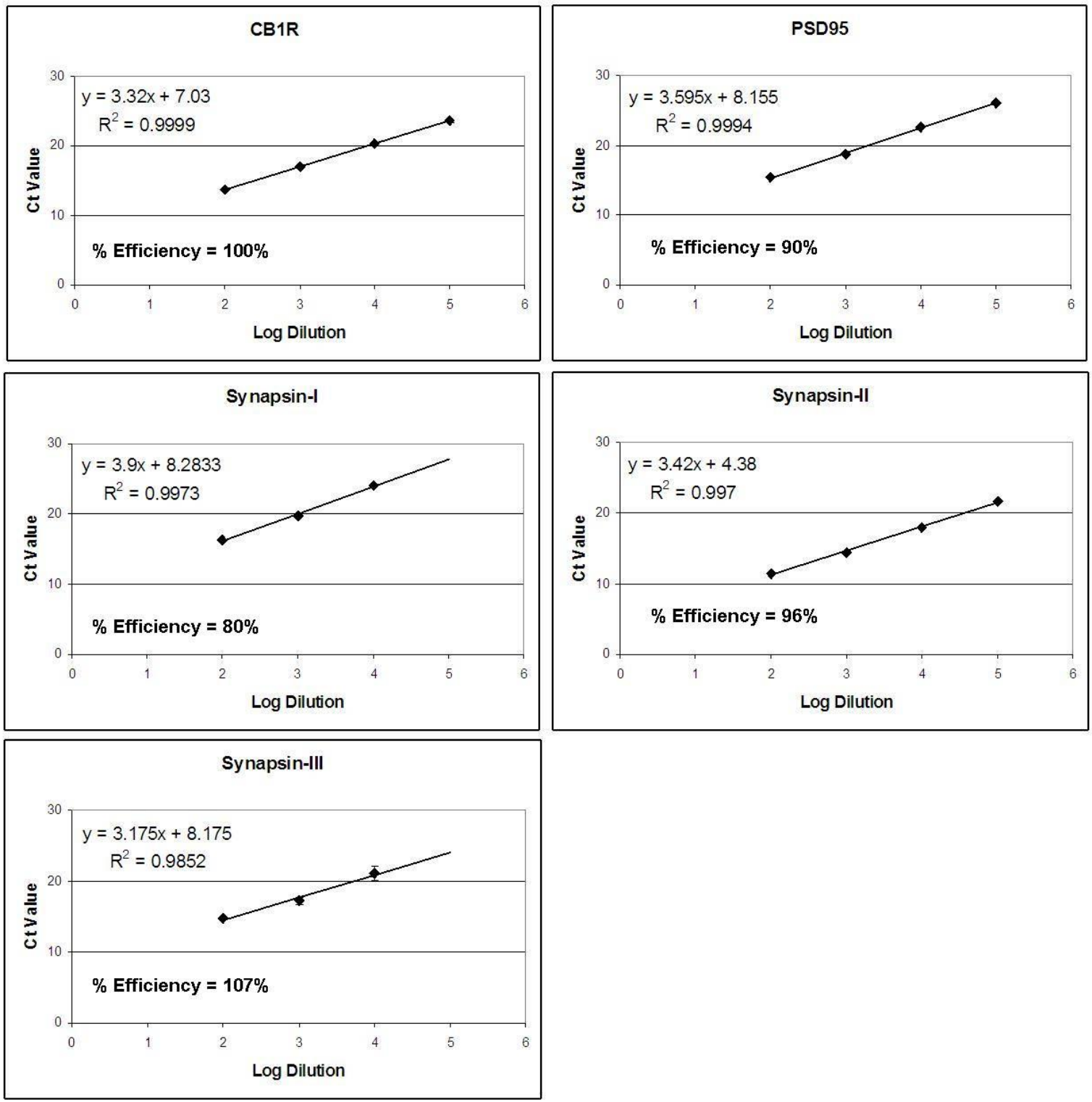

Figure 15: Efficiencies of the primers used to assess markers of plasticity. Purified PCR products were diluted and amplified in duplicate, and the mean $\mathrm{Ct}$ value plotted against the log of the dilution. The slope of the best fit line was used to calculate primer efficiencies (displayed on the graph). $R^{2}$ values demonstrate the fit of the trend line to the data. 


\subsubsection{Melt Curve Analysis}

Following the completion of the final PCR amplification cycle (representative sample trace shown in Fig. 16A), melt curve analysis was used to determine the purity of PCR products. A single product is illustrated by a single peak when the rate of fluorescence change is plotted against the temperature. The temperature at which the peak occurs is determined by the base composition of the product.

A single sharp peak was observed in the melt curves for $\mathrm{CB} 1 \mathrm{R}\left(86^{\circ} \mathrm{C}\right)$, cyclophilin A $\left(84^{\circ} \mathrm{C}\right)$, synapsin-I $\left(89.5^{\circ} \mathrm{C}\right)$, synapsin-II $\left(83^{\circ} \mathrm{C}\right)$ and synapsin-III $\left(85^{\circ} \mathrm{C}\right)$, indicating the presence of a single PCR product; whereas, the melt curve of PSD95 showed a more diffuse peak (between $80.5-90.5^{\circ} \mathrm{C}$ ). This may indicate the generation of spurious PCR product by the PSD95 primer pair. All primer sets appeared to have at least some level of primer dimer, as suggested by the presence of a small amount of product between $73-85^{\circ} \mathrm{C}$ (Fig. 16B). PCR products were resolved and visualised using agarose gel electrophoresis to confirm the identity on the basis of size (Fig. 16C). All primer sets amplified a single product of the expected size. In addition the PSD95 primer pair produced some primer dimer, and the synapsin-I primer pair appeared to also amplify a small amount of off-target product of approximately $500 \mathrm{bp}$. Because these products were scarce compared to the expected product, and because analysis on samples with Ct values between 20 - 30 cycles, as seen for these transcripts, have been reported to be relatively unaffected by primer-dimer and non-specific product formation, the contribution of these products to the analysis were considered to be negligible (Peters, Helps et al. 2004). 

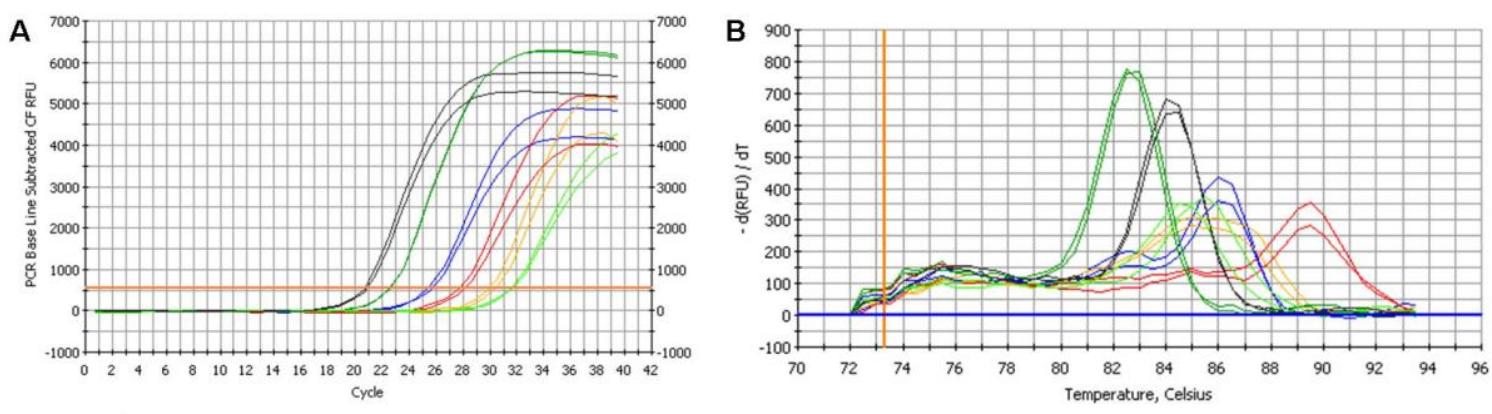

C
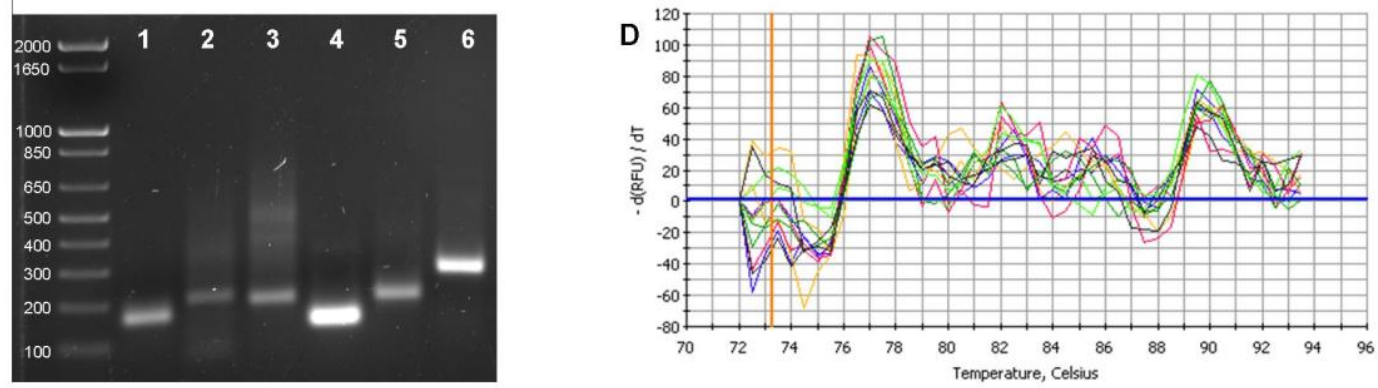

Figure 16: Representative real-time PCR data used to assess plasticity. (A) A representative trace image of data acquired by real-time PCR for target genes used in this chapter. All genes (Cyclophilin A, black; CB1R, blue; PSD95, gold; synapsin-I, red; synapsin-II, dark green; synapsinIII, lime) were analysed in duplicate to ensure the accuracy of the results. (B) Melt-curve profile analysis of these samples revealed single sharp peaks for CB1R $\left(86^{\circ} \mathrm{C}\right)$, synapsin-I $\left(89.5^{\circ} \mathrm{C}\right)$, synapsin-II $\left(83^{\circ} \mathrm{C}\right)$, synapsin-III $\left(85^{\circ} \mathrm{C}\right)$ and cyclophilin $\mathrm{A}\left(84^{\circ} \mathrm{C}\right)$ while PSD95 shows a more diffuse peak $\left(85^{\circ} \mathrm{C}\right)$. Low levels of product were also detected at $73-85^{\circ} \mathrm{C}$ for all primer pairs. (C) PCR products were validated using agarose gel electrophoresis (lane 1, CB1R; lane 2, PSD95; lane 3, synapsin-I; lane 4, synapsin-II; lane 5, synapsin-III; lane 6, cyclophilin A). (D) Melt-curve profile analysis of negative controls (PCR reactions completed with template replaced with either $\mathrm{dd}_{2} \mathrm{O}$ or equi-molar non-reverse transcribed RNA) showed no specific PCR product for any of the genes analysed.

For each primer pair, PCR amplification and melt curve analysis of negative controls (in which template was replaced with either $\mathrm{ddH}_{2} \mathrm{O}$ or equi-molar nonreverse transcribed RNA) detected no specific PCR product (Fig. 16D). These data rule out the possibility that contaminating PCR product or genomic DNA were amplified during the PCR reaction. 


\subsubsection{Chronic THC Exposure Attenuates Transcription of Plasticity Markers}

For each transcript, mean $\Delta \mathrm{Ct}$ values were determined for each treatment and training group. From the $\Delta \mathrm{Ct}$ values, the $\Delta \Delta \mathrm{Ct}$ values were calculated relative to vehicle-treated untrained control animals. Calculated $\Delta \mathrm{Ct}$ values and $\Delta \Delta \mathrm{Ct}$ values are presented in (Table 5) and (Fig. 17), respectively.

Two-way ANOVA (training $x$ transcript) found that training of vehicle-treated, but not THC-treated animals in the radial maze increased mRNA expression $(p<0.001)$, especially the expression of synapsin-I $(p<0.001)$ and synapsin-III $(p<0.01)$. These transcripts increased by $136 \%$ (corresponding to $1.24 \Delta \Delta \mathrm{Ct}$ units) and $104 \%$ (corresponding to $1.03 \Delta \Delta \mathrm{Ct}$ units), respectively. One-way ANOVA detected significant differences between treatment and training groups for synapsin-I $(p<0.05)$ and synapsin-III $(p<0.01)$, that were increased in vehicletreated but not THC-treated animals by training in the radial maze $(p<0.05$ and $p<0.01$, respectively), supporting the findings of Two-way ANOVA above. A $54 \%$ (corresponding to $0.62 \Delta \Delta \mathrm{Ct}$ units) increase in PSD95 ( $p=0.059$ ), and a $62 \%$ (corresponding to $0.7 \Delta \Delta \mathrm{Ct}$ units) increase in $\mathrm{CB} 1 \mathrm{R}(p=0.066)$ upon training in vehicle-treated animals, however, marginally failed to achieve significance, although strong trends were observed. Consequently, Two-way ANOVA (treatment $x$ transcript) found that vehicle-treated animals trained in the radial maze had higher transcript levels than trained THC-treated animals $(p<0.001)$. This was especially evident for PSD95 levels, that were $111 \%$ higher (corresponding to $1.08 \Delta \Delta \mathrm{Ct}$ units) in vehicle-treated trained animals $(p<0.01)$. No effects of THC-treatment on transcript levels, however, were detected for home cage, untrained animals (Fig. 17). 


\begin{tabular}{|c|c|c|c|c|}
\hline \multirow[b]{2}{*}{ Target } & \multicolumn{2}{|c|}{ Trained Animals } & \multicolumn{2}{|c|}{ Untrained Animals } \\
\hline & $\begin{array}{l}\text { Vehicle- } \\
\text { Treated }\end{array}$ & THC-Treated & $\begin{array}{l}\text { Vehicle- } \\
\text { Treated }\end{array}$ & THC-Treated \\
\hline CB1R & $\begin{array}{c}3.64 \pm 0.13 \\
(1.62 X)\end{array}$ & $\begin{array}{c}4.16 \pm 0.21 \\
(1.09 X)\end{array}$ & $4.34 \pm 0.17$ & $\begin{array}{c}4.28 \pm 0.32 \\
(1.04 X)\end{array}$ \\
\hline PSD95 & $\begin{array}{c}8.09 \pm 0.26 \\
(1.54 X)\end{array}$ & $\begin{array}{c}9.17 \pm 0.52 \\
(0.93 X)\end{array}$ & $8.71 \pm 0.2$ & $\begin{array}{c}9.06 \pm 0.11 \\
(0.78 X)\end{array}$ \\
\hline Synapsin-I & $\begin{array}{c}5.33 \pm 0.23 \\
(2.36 X)\end{array}$ & $\begin{array}{c}5.76 \pm 0.16 \\
(1.51 X)\end{array}$ & $6.57 \pm 0.38$ & $\begin{array}{c}6.35 \pm 0.37 \\
(1.16 X)\end{array}$ \\
\hline Synapsin-II & $\begin{array}{c}1.15 \pm 0.09 \\
(1.36 X)\end{array}$ & $\begin{array}{c}1.51 \pm 0.19 \\
(1.16 X)\end{array}$ & $1.59 \pm 0.21$ & $\begin{array}{c}1.72 \pm 0.33 \\
(0.91 X)\end{array}$ \\
\hline Synapsin-III & $\begin{array}{c}9.37 \pm 0.16 \\
(2.04 X)\end{array}$ & $\begin{array}{c}9.86 \pm 0.13 \\
(1.57 X)\end{array}$ & $10.4 \pm 0.29$ & $\begin{array}{c}10.51 \pm 0.3 \\
(0.93 X)\end{array}$ \\
\hline
\end{tabular}

\footnotetext{
Table 5: Relative gene expression levels between treatment and behavioural groups for plasticity markers. Data are mean $\pm \mathrm{SEM}$ of the $\Delta \mathrm{Ct}$ values. Fold change relative to untrained animals of the same treatment (for trained animals), or vehicle-treated animals (for untrained THCtreated animals) are shown in parenthesis.
} 


\section{mRNA Expression Relative to Untrained Controls}

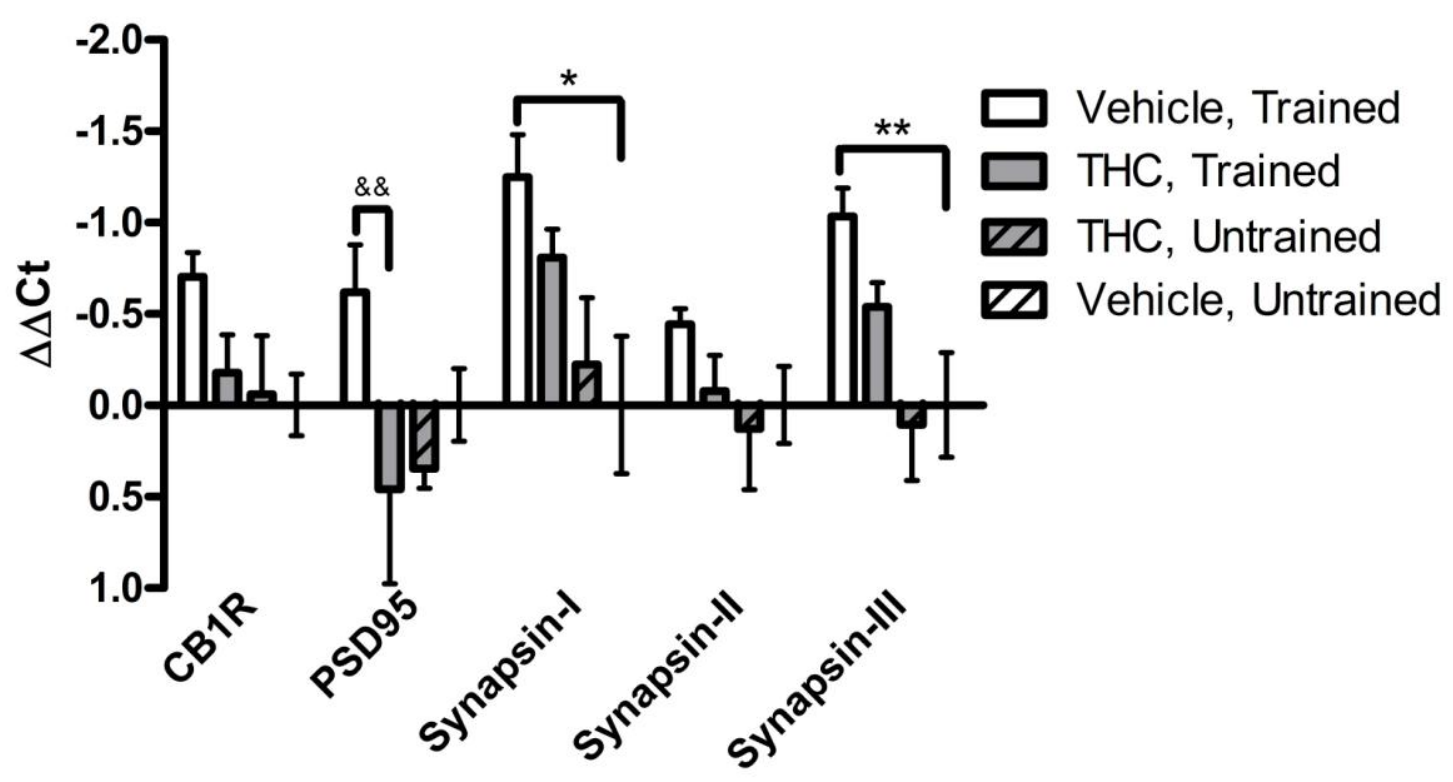

Figure 17: THC attenuates the transcription of markers involved in synaptic plasticity.

Training in the radial maze increased the transcription of markers analysed overall, and although specific increases in transcript levels were only observed for synapsin-I and synapsin-III transcripts, those for CB1R and PSD95 only just failed to achieve statistical significance. While training also appeared to increase transcription in THC-treated animals, this relationship was not statistically significant, and indeed, a significant difference was detected between the transcript levels in THCtreated and vehicle-treated animals. THC did not affect the baseline expression of transcripts. Data are presented as the mean $\Delta \Delta \mathrm{Ct}$ values \pm SEM relative to untrained vehicle-treated animals, with the $y$-axis inverted for presentation purposes. ${ }^{*} p<0.05$ by One-way ANOVA, ${ }^{* *}{ }^{\# \#} p<0.01$ by Oneway ANOVA/Two-way ANOVA.

\subsubsection{Chronic THC Exposure Prevents Adaptive Reponses in Synaptic Plasticity}

Hippocampal protein lysates were immunoblotted to determine if the trends observed in the mRNA data were corroborated by similar changes in protein expression (Fig. 18). Two-way ANOVA (training x protein) found that training significantly reduced protein expression in vehicle-treated animals $(p<0.001)$, and that CB1R $(32 \%, p<0.05)$ and PSD95 $(31 \%, p<0.05)$ were especially reduced by 
training. No reduction was observed in THC-treated animals trained in the radial maze. One-way ANOVA detected significant differences between the mean expression levels of both CB1R $(p<0.001)$ and PSD95 $(p=0.05)$ between treatment and training groups. The levels of both CB1R and PSD95 were reduced by training in vehicle-treated animals ( $p<0.05$ for both). However, THC-treatment reduced the expression of CB1R $(53 \%, p<0.001)$, but not PSD95 (9\%) in untrained, home cage control animals. The trends observed in the PSD95 dataset appeared to be consistent with trends in the levels of synapsin-I, including a $27 \%$ reduction of expression upon training that was not observed in THC-treated animals, although the synapsin-I changes were not statistically significant $(p=0.25)$. Two-way ANOVA (treatment $x$ protein) detected a significant interaction effect when either trained or untrained THC-treated and vehicle-treated animals were compared, making the data difficult to interpret. However, the reduction of CB1R in THC-treated untrained animals appeared to entirely account for the interaction effect, because when CB1R data were excluded from the analyses no interactions were detected. Vehicle-treated animals had lower levels of PSD95 and synapsin-I than THCtreated animals trained in the radial maze $(p<0.05)$; whereas, no differences in these levels were observed between THC-treated and vehicle-treated untrained, home cage control animals. 


\section{A Protein Abundance Relative to Untrained Controls}

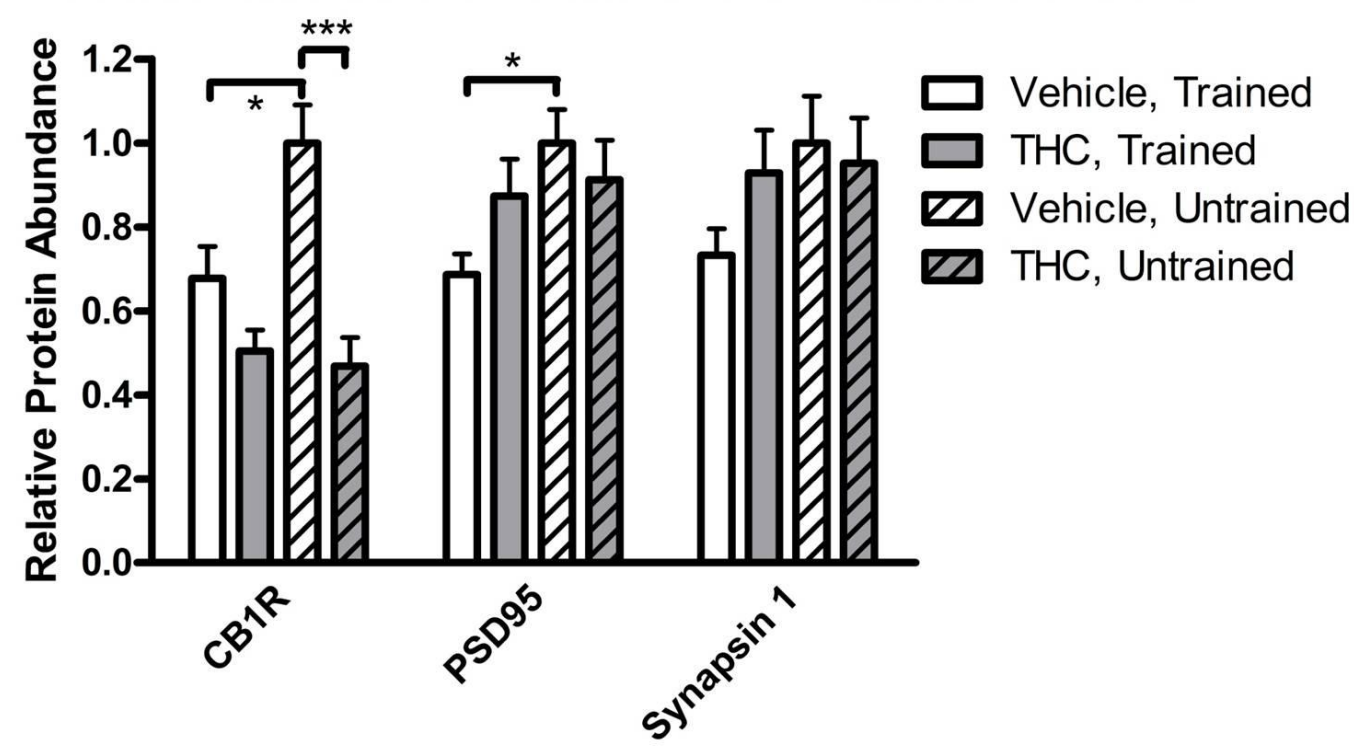

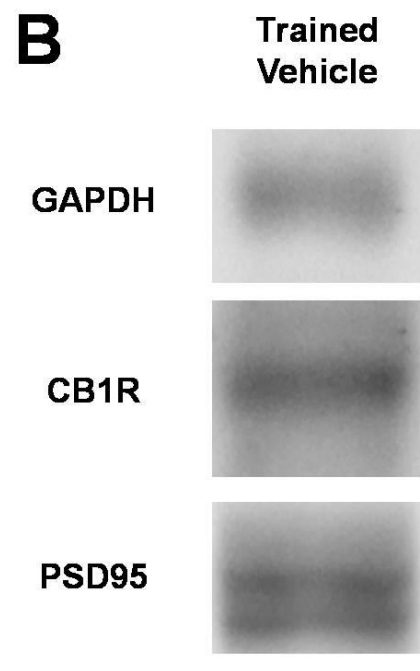
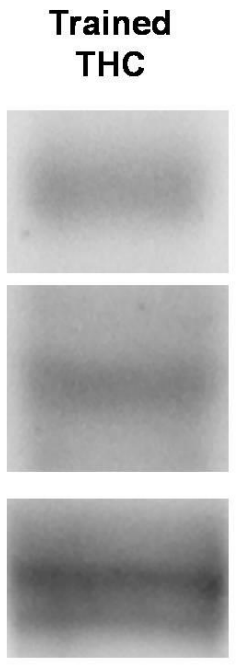

Synapsin-I

\section{$\left(\frac{100}{10}\right.$}

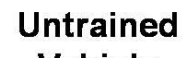

Vehicle
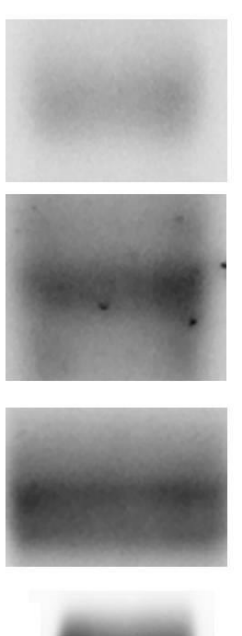

\section{Untrained}

THC
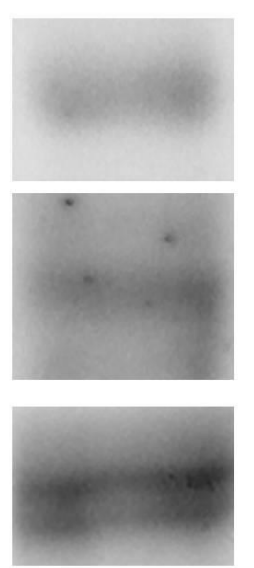

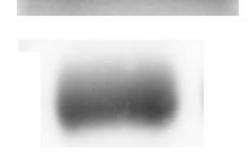

Figure 18: The training-induced reduction in synaptic protein abundance is blocked by THCtreatment. (A) Training in the radial maze reduced the abundance of PSD95 and CB1R in vehicle-, but not THC-treated animals. A similar reduction in synapsin-I in vehicle-treated animals only upon training did not achieve statistical significance. THC had no effect on synapsin-I and PSD95 levels in untrained animals, but significantly reduced the expression of CB1R. Data are mean \pm SEM; * $p<0.05,{ }^{* * *} p<0.001$ by one-way ANOVA. (B) Fluorescent scans of representative immunostained bands, for CB1R, PSD95 and synapsin-I. 


\subsection{Discussion}

While the behavioural deficits associated with cannabis use are widely accepted, few studies have investigated the molecular changes that may account for this behavioural disruption. This chapter has investigated whether the impairment of learning in adolescent animals (shown in Chapter 3 ) is correlated with impairment of synaptic plasticity. Synaptic plasticity, including functional plasticity (LTP) as well as structural plasticity (the recruitment of new and rearrangement of existing synapses), is required to perform complex brain functions such as learning and memory (Bailey and Kandel 1993; Moser 1999; Martin, Grimwood et al. 2000). Analyses were conducted on animals that were both trained in the radial maze, and those that were left untrained. This allowed the investigation of whether synaptic plasticity in response to training in a spatial task is especially sensitive to disruption by THC, or rather whether the effects of THC on the basal state culminate in behavioural impairment

Synaptic plasticity was investigated in the hippocampus because it represents the brain structure most relevant to spatial learning and memory (Gallagher and Holland 1992; Moser, Moser et al. 1993; Skinner, Martin et al. 1994; Cho, Friedman et al. 1999). A range of plasticity markers were included in the analysis. The synapsins are a group of phosphoproteins associated with presynaptic terminals (Sudhof, Czernik et al. 1989; Feng, Chi et al. 2002; Pieribone, Porton et al. 2002), and thus are good markers for presynaptic connections. Additionally, synapsin-I is strongly implicated in the induction and maintenance of LTP (Nayak, Moore et al. 1996). PSD95 is a postsynaptic marker, tightly correlated with postsynaptic sites, although it, like synapsin-I, is also involved in the induction and maintenance of LTP (Kennedy 2000). Thus, PSD95 and synapsin-I are markers of both structural and functional plasticity. Finally, CB1R is the main cannabinoid receptor in the CNS (Trezza, Cuomo et al. 2008). CB1R is strongly implicated in the mechanism of LTD (Heifets and Castillo 2009), inhibiting the release of GABA, and leading to the induction and maintenance of LTP, required for learning (Martin, Grimwood et al. 2000). 
Training in the RAM enhanced the transcription of markers of synaptic plasticity in vehicle-treated animals, and these increases were attenuated by THC-treatment. The levels of synapsin-I and synapsin-III were especially affected by training in vehicle-treated animals, and vehicle-treated animals generally had higher levels of all transcripts than THC-treated animals trained in the RAM. This was particularly evident for the levels of PSD95. Importantly, the impairment of learning associated transcription by THC was observed in the absence of any difference in the transcription of these markers in animals that were not trained in the RAM. Thus, the behavioural impairment associated with THC-treatment during adolescence is due to the sensitivity of synaptic plasticity during learning to $\mathrm{THC}$, rather than because of the effects of THC in the basal (unlearning) state.

Increases in transcription of synapsin-I and PSD95 upon training presented in this chapter suggest an increase in structural and functional plasticity upon learning. Memory consolidation and long-term memory require de novo protein synthesis from learning induced changes in gene expression (Davis and Squire 1984; Bailey and Kandel 1993). The data in this chapter showing changes in the expression of these genes are supported by similar protein changes in previous studies (GomezPinilla, So et al. 2001; Skibinska, Lech et al. 2001). Few studies, however, have investigated the effect of THC on synaptic plasticity. In one study, behavioural impairment in adulthood following adolescent THC treatment was associated with reduced levels of the presynaptic protein VAMP2, and PSD95 in the hippocampus (Rubino, Realini et al. 2009). Synapsin-I and VAMP2 are roughly analogous markers, as both are vesicle-associated proteins found together at presynaptic sites and are involved in increasing neurotransmitter release during LTP (Nayak, Moore et al. 1996; Sanes and Lichtman 1999; Schoch, Deak et al. 2001). Rubino et al. concluded that adolescent THC treatment caused lasting impairment of structural and functional plasticity, resulting in cognitive impairment in adulthood (Rubino, Realini et al. 2009). The data presented in this chapter support a similar impairment in structural and functional plasticity when testing is concurrent with THC-treatment in adolescence. Furthermore, they indicate that plasticity associated with learning, rather than the basal state, is especially sensitive to disruption by THC. This finding of the present study refines the nature of the 
impairment reported by Rubino et al., since in their study, no comparison was made with untrained animals.

CB1R mRNA was increased by training in the radial maze in vehicle-treated animals. Learning enhances LTP in the hippocampus (Martin, Grimwood et al. 2000), and this is mediated by increasing the expression of a number of molecules responsible to the induction and maintenance of LTP (Sanes and Lichtman 1999). Although a number of studies have implicated CB1R as a molecule important in LTP (Collins, Pertwee et al. 1995; Terranova, Michaud et al. 1995; Misner and Sullivan 1999), none have demonstrated any alteration in its expression upon learning. Subjecting animals to enriched environment or exercise, however, both of which enhance hippocampal dependent learning, have been shown to increase hippocampal CB1R mRNA levels (Wolf, Bick-Sander et al. 2010). An increase in CB1R may contribute to increased LTP required for learning. Higher levels of CB1R presumably cause a greater inhibition of GABAergic interneurons in response to endocannabinoid signalling from the postsynaptic neuron, thus facilitating a greater DSI and the induction of LTP. THC-treated animals trained in the RAM showed no enhancement in CB1R mRNA levels, and this may impair learning by reducing the likelihood of LTP induction. This effect is likely compounded by the down regulation of CB1R protein in an apparent tolerance response observed in THC-treated animals in this study. Indeed, it has been suggested that cannabinoids impair LTP by preventing the attainment of sufficient levels of neurotransmitter required for LTP induction (Sullivan 2000), and inhibition of LTD would increase the threshold required for LTP induction.

Overall, the results of the mRNA analyses indicate that deficits in adolescent learning following THC-treatment can likely be attributed to the effects of THC on plasticity responses to learning, rather than any effects on the basal state that culminate in cognitive impairment. When protein levels were analysed, training of vehicle-treated, but not THC-treated animals in the RAM reduced the expression of synaptic proteins, especially CB1R and PSD95. Once CB1R data were 
excluded from the analyses, THC had no effect on base line expression of synapsin-I and PSD95, and the abundance of these proteins were lower in vehicle-treated, but not THC-treated animals trained in the RAM. This data suggests a reduction in synaptic connections in the hippocampus upon training in adolescents is blocked by THC-treatment. CB1R data were excluded from this analysis because the decreases in the levels of this protein in THC-treated animals appeared to be mediated by the development of tolerance to the ligand, rather than the effects of the drug on synaptic plasticity. Indeed, the development of tolerance to ligands such as THC can be achieved by receptor degradation (Hsieh, Brown et al. 1999; Leterrier, Bonnard et al. 2004; Martini, Waldhoer et al. 2007) and has been shown in adolescent animals previously (Breivogel, Childers et al. 1999; Rubino, Vigano et al. 2008).

Interestingly, cognitive impairment resulting from THC-treatment has been associated with enhanced translation (Puighermanal, Marsicano et al. 2009) that has the potential to mask any reduction of plasticity markers by training seen in vehicle-treated animals. This possibility is however unlikely for several reasons. Firstly, memory consolidation requires de novo protein synthesis in the period immediately following training (Inda, Delgado-Garcia et al. 2005), and this was the period Puighermanal et al (2009) administered THC within in their study. Protein translation was elevated for up to 4 hours following treatment, and the cognitive impairment measured 24 hours later could be reversed using a protein synthesis inhibitor. In this study THC was administered 19 hours before the completion of training. This is significantly greater than the 4 hours after THC-treatment where de novo protein synthesis is particularly important (Inda, Delgado-Garcia et al. 2005) and when translation is enhanced by THC (Puighermanal, Marsicano et al. 2009). Furthermore the expression of CB1R was significantly reduced by the chronic THC-treatment regimen used in this study. This is significant because the increase in translation was mediated via CB1R (Puighermanal, Marsicano et al. 2009), and reduced receptor density could be expected to minimise this effect of THC. Finally, THC appeared to have no effect on protein levels in animals not trained in the radial maze, arguing against any increase in translation by THCtreatment. As such the potential effects of $\mathrm{THC}$ on protein translation appear to 
have been mitigated by the experimental design of this study. Thus the protein data support the conclusions drawn from the mRNA data, as THC-treatment prevented plasticity in response to learning, rather than the drug affecting the basal state culminating in cognitive impairment. It is not immediately clear however, why training of vehicle-treated animals is associated with increased transcription, but reduced protein levels for these plasticity markers. Interestingly, reductions in synaptic protein, such as those seen here upon training, are routinely observed during neurodevelopment in the adolescent period of a number of species.

During adolescence, synaptic connections in the hippocampus and other brain regions are refined into their adult neural architecture. Significant reductions in spine density and associated neurotransmitter systems, including reductions in CB1R levels, are observed in this pruning mechanism in the hippocampus (Spear 2000; Zehr, Nichols et al. 2008). Changes such as these are associated with the emergence of adult patterns of brain activation during task performance and the improvement of many cognitive functions (Spear 2000). Significantly, spatial learning and memory skills develop throughout the adolescent period, and this is related to cellular changes in the hippocampus (Uysal, Dayi et al. 2010). Adolescence can thus be thought of as a plastic period that precedes the emergence of adult cognitive processes. Surprisingly, whether learning, known to elicit increases in structural and functional plasticity (Bailey and Kandel 1993; Moser 1999; Martin, Grimwood et al. 2000), enhances the developmental plasticity associated with the adolescent period has not been investigated. The results of this chapter show an impairment of synaptic plasticity by THC treatment in adolescence, although the direction of change was different at the mRNA and protein levels. It is tempting to speculate that the adolescent age period, in which these data were collected, may represent a unique paradox in which these different results may be explained.

The data of this chapter show that training in the radial maze facilitated reductions in synaptic proteins similar to that reported during adolescent neurodevelopment. 
This may represent an acceleration of adolescent development corresponding to enhanced spatial learning. This is because adolescence is associated with significant developmental changes, including reductions in pre and postsynaptic connections (Spear 2000), and because spatial learning, that develops during the adolescent period (Uysal, Dayi et al. 2010), was greater in animals that appeared to eliminate their synapses in response to training in the RAM. That reductions in these proteins were not seen in THC-treated animals may suggest that THC prevents accelerated adolescent neurodevelopment associated with spatial training. Interestingly, an impairment of synaptic elimination by THC has been shown previously, as network driven synapse loss was prevented by THC using an in vitro model of high neural activity (Kim, Waataja et al. 2008). Additionally, training of vehicle-treated, but not THC-treated animals in the RAM increased the mRNA levels of these markers. Decreases in protein levels, possibly representing synaptic elimination, appeared to significantly outweigh any de novo protein synthesis associated with increased mRNA levels upon training. As such, decreased protein and increased mRNA may represent two plasticity mechanisms operating simultaneously in adolescent learning animals; developmental plasticity, and synaptic plasticity, respectively. Thus, THC-elicited disruption of the balance between synaptic elimination and synaptic establishment during times of learning may represent a novel set of molecular circumstances in which adolescent learning is impaired simultaneously at two levels of plasticity. However, this proposal is merely speculation as the data presented here are unable to confirm this possibility and more investigation is required.

These data build significantly on the limited earlier studies that have investigated the molecular basis of learning and memory impairment that is associated with cannabis use. The results suggest that an increase in CB1R may be important for learning, and that increases in CB1R mRNA are blocked by THC. This effect may be compounded by the induction of CB1R degradation upon ligand binding in THC-treated animals. Additionally, the data suggest that the adolescent behavioural deficits caused by THC exposure are at least partly attributable to impairment of structural and functional plasticity associated with training. Similar impairments in adults have been reported following adolescent THC exposure 
(Rubino, Realini et al. 2009). The data in this chapter extend these previous findings by demonstrating a particular sensitivity of synaptic plasticity to the effects of THC, rather than effects of THC in the basal sate culminating in learning impairment. The relative contribution of these impairments was previously unclear. Finally, the data may suggest that adolescent neurodevelopment is accelerated by spatial learning, and that this acceleration was impaired by THC-treatment. The data are however insufficient to absolutely support this hypothesis, and further investigation is required.

Significant changes in mRNA levels for synapsin-III were also seen in this study. Training in the radial maze increased the expression of synapsin-III, and this was prevented by THC treatment. While located at presynaptic sites, synapsin-III is predominantly located on maturing newborn neurons (Pieribone, Porton et al. 2002; Kao, Li et al. 2008), where it is concentrated in early growth cones and is involved in axonal outgrowth and differentiation (Ferreira, Kao et al. 2000). Despite the apparent role of synapsin-III in neurogenesis, and the fact that learning increases neurogenesis in the hippocampus (Gould, Beylin et al. 1999), these are the first data that demonstrate that learning enhances the expression of synapsinIII. Furthermore, these data suggest that deficits in neurogenesis, in addition to synaptic plasticity, may contribute to cognitive impairment in THC-treated animals. Indeed, the behaviour of THC-treated animals trained in the RAM (see Chapter 3) shares similar features to synapsin-III knockout animals, including subtle deficits in reference memory and a reduced reliance on spatial search strategies (Porton, Rodriguiz et al. 2010). The effects of THC on neurogenesis are investigated in Chapter 5. 


\section{Chapter 5 Effects of THC on Hippocampal Neurogenesis}

\subsection{Introduction}

Neurons are continuously generated in discrete regions of the adult brain, including the dentate gyrus of the hippocampus (Altman and Das 1965). A population of neural stem cells (NSCs) lines the inner face of the dentate gyrus (Denise, Garcia et al. 2004), and these cells divide to generate neuroblasts (Seri, Manuel et al. 2004). In normal circumstances, many of these neuroblasts are lost by apoptosis (Sun, Winseck et al. 2004). Survival of these newborn neurons, however, is enhanced by (Gould, Beylin et al. 1999), and indeed required for (Shors, Miesegaes et al. 2001), performance in hippocampal-dependent learning tasks. Neurons that survive become functionally integrated into the hippocampus, extending processes towards the molecular layer and to the CA3 region of the hippocampus (Deng, Aimone et al. 2010). The contribution of these synapses to the hippocampal circuit is thought to be an important mechanism of increased structural plasticity (see Chapter 4) during learning (Bailey and Kandel 1993; Moser 1999). However, the major role of adult hippocampal neurogenesis is thought not to be its contribution to structural plasticity, but rather to a process called pattern separation.

Increased survival of neuroblasts during learning appears restricted to neurons able to receive functional input; whereas, those that are not able to are eliminated (Deng, Aimone et al. 2010). In this way, the targets innervated by newborn neurons are enriched for connections that support the memory learnt during training (Dupret, Fabre et al. 2007). The hyperexcitability associated with the early age of newborn neurons that are selected during learning allows them to be preferentially incorporated into multiple memory circuits. In this way, memories activating similar sets of granule cells in the dentate gyrus can result in the activation of very different efferent neural networks. This allows related memories to be recalled together, but prevents interference between these memories (Deng, 
Aimone et al. 2010). Thus adult hippocampal neurogenesis is an important plasticity mechanism contributing to learning and memory in various tasks including that used in the present thesis, the RAM, in which similar cues are used to determine the location of rewarded and non-rewarded arms.

Neural progenitor cells contain a functional endocannabinoid signalling system (Aguado, Monory et al. 2005; Jiang, Zhang et al. 2005; Aguado, Palazuelos et al. 2006; Mulder, Aguado et al. 2008) including the CB1R receptor, that is maintained on maturing granule neurons (Wolf, Bick-Sander et al. 2010). Activation of CB1R receptors increases neural progenitor cell proliferation; whereas, knockout of CB1R decreases proliferation (Jin, Xie et al. 2004; Aguado, Monory et al. 2005; Jiang, Zhang et al. 2005; Aguado, Palazuelos et al. 2006; Mulder, Aguado et al. 2008). Furthermore, cannabinoids appear to delay the maturation of neuroblasts (Rueda, Navarro et al. 2002; Galve-Roperh, Aguado et al. 2006), although they have no effect on the survival of newborn neurons in the absence of training (Wolf, Bick-Sander et al. 2010). Thus, cannabinoids appear able to influence neurogenesis from neural progenitor cell proliferation through to maturation of adult born neuroblasts. Curiously, the research that has investigated the effects of cannabinoids on neurogenesis has neglected to determine whether enhanced neurogenesis associated with learning is affected by cannabinoid drugs, despite the important role played by neurogenesis in learning.

This chapter investigates whether changes in neurogenesis associated with learning are affected by treatment with THC. The effect of THC on learningenhanced neuronal survival was tested using BrdU labelling to trace newborn neurons. Other aspects of neurogenesis were investigated by immunohistochemistry (IHC) staining using specific markers. Sox2 was used as a marker to determine the number of neural stem cells in the hippocampus that are responsible for producing young neurons (Graham, Khudyakov et al. 2003; Ferri, Cavallaro et al. 2004; Suh, Consiglio et al. 2007; Favaro, Valotta et al. 2009). The level of proliferation in the hippocampus was assessed using the marker Ki67 that stains all proliferating cells (Scholzen and Gerdes 2000). The overall number of 
newborn neurons was assessed using the markers doublecortin (DCX) and the post-translational addition of polysialic acid (PSA) to the neural cell adhesion molecule (NCAM). Expression of both DCX and PSA-NCAM is evident shortly after birth, does not co-localise with markers of neural stem cells and is downregulated in mature neurons (von Bohlen und Halbach 2007). Finally, the expression of brain-derived neurotrophic factor (BDNF), a cytokine that has previously been shown to promote the differentiation and survival of neurons, was measured to determine whether THC elicited any change in the level of this important neurotrophic factor (Memberg and Hall 1995; Palmer, Takahashi et al. 1997; Sairanen, Lucas et al. 2005). Analyses were performed in both trained and untrained animals to determine if any effects of THC were attributable to impairment of adaptive responses specifically, or rather to the effects of THC on the basal, untrained state.

\subsection{Methods}

\subsubsection{Animals}

The basic experimental design was as described in Chapter 4. Briefly, adolescent rats were treated with $\mathrm{THC}$ for 15 days, and the effect of $\mathrm{THC}$ on neurogenesis in animals that either had or had not been trained in the radial maze during this time was assessed. This training period coincided with the early emergence of behavioural differences between THC-treated and vehicle-treated animals (see Chapter 3). Hippocampal tissue was collected from these animals as described previously (see section 2.4 and 2.5). 


\subsubsection{Molecular Analyses and Experimental Design}

A combination of $\mathrm{IHC}$, Western blotting and real-time PCR were used to assess the effects of THC on neurogenesis in the hippocampus. Survival of a cohort of BrdU-labelled nuclei can be used to assess the effect of THC on the survival of neurons born at the beginning of training. BDNF, Sox2 and Ki67 present a snapshot of neurogenesis at the time of sacrifice. PSA-NCAM and DCX are expressed for 3 - 4 weeks following neuronal birth (von Bohlen und Halbach 2007; Deng, Aimone et al. 2010), and thus their presence indicates the birth and survival of neurons in the weeks before as well as during the 2-week experimental window used in this study. This combination of analyses allows the simultaneous investigation of several stages of neurogenesis.

\subsubsection{Western Blotting and Real-Time PCR}

A variety of neurogenic markers were used in this chapter. Sox2 was used as a marker of NSCs, and Ki67 was used as a proliferative marker. PSA-NCAM was used as a marker of young neurons. The addition of PSA to the NCAM is a posttranslational modification, and thus cannot be assessed by real-time PCR. Instead, DCX mRNA levels were assessed, as PSA-NCAM and DCX are considered equivalent markers of young neurons (von Bohlen und Halbach 2007). In addition, BDNF was assessed because of its role in promoting the generation and survival of newborn neurons. The antibodies used for Sox2 (43 kDa), PSA-NCAM ( 300 $\mathrm{kDa})$, and BDNF ( 28kDa) detected single bands of the expected size. The Ki67 antibody detected a doublet of $\sim 345$ and $\sim 395 \mathrm{kDa}$ as expected, and both of these bands were used in the densitometry analysis. The analyses of protein and mRNA quantities were performed as previously described (see section 2.4). 


\subsubsection{Immunohistochemistry}

Animals were injected with BrdU on the eve of training to label a cohort of newborn neurons (see section 2.3). Fifteen days later, animals were sacrificed and processed for the detection of surviving BrdU-labelled neurons by IHC and DAB staining (see section 2.5). The analysis was based on a method using matched sections and systematic random sampling (Fig. 19). Every fifth section of $20 \mu \mathrm{m}$ through a $1200 \mu \mathrm{m}$ segment of the dorsal hippocampus was processed for the enumeration of surviving BrdU-labelled neurons from the cohort of labelled neurons. Analysed sections were $100 \mu \mathrm{m}$ apart, ensuring the same cell was not counted twice. All BrdU ${ }^{+}$cells were counted throughout the entire length of the dentate gyrus and thickness of the section, and binned as being in either the hilus, the subgranular zone (SGZ, defined as the zone encompassing a 2-cell nuclei width either side of the interface between the hilus and dentate gyrus) or the granule cell layer (GCL, defined as all cells of the dentate gyrus not included in the SGZ, Fig. 19B). These regions were counted in both the superior and inferior blades dentate gyrus. Where the blades met, the superior and inferior dentate gyrus was separated by drawing a line through the apex of this structure (Jiang, Zhang et al. 2005). 

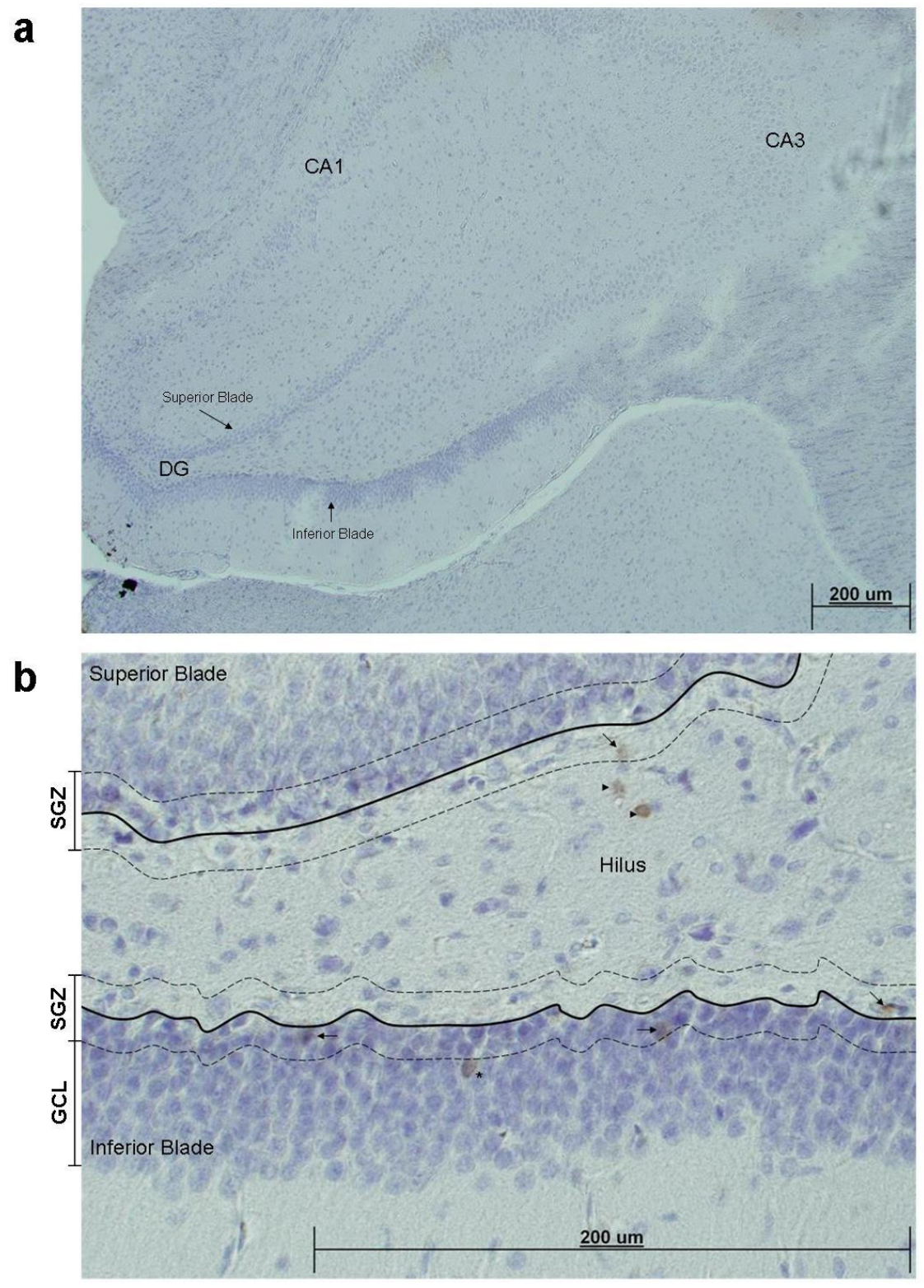

Figure 19: Method for the enumeration of $\mathrm{BrdU}^{+}$nuclei in the hippocampus. A) Cell counting was performed using systematic sampling. The first appearance of the superior blade of the dentate gyrus, before the merging of the superior and inferior blades, was used as a common morphological feature defining the first section to be analysed. B) $\mathrm{BrdU}^{+}$nuclei (brown) were counted in the SGZ (arrows) and GCL (asterisk) of both the superior and inferior blades of the dentate gyrus, as well as the hilus (arrow heads). The interface between the dentate gyrus and the hilus is shown by a solid line, and a 2-cell-bodywide zone along this interface was considered the SGZ. The area between the superior and inferior blades of the dentate gyrus that was not included in the SGZ was considered the hilus; whereas, the area of the dentate gyrus not included in the SGZ was considered the GCL. 


\subsubsection{Statistical Analyses}

Western blot and real-time PCR analyses were conducted as described previously (see section 2.6) using the same samples and number of observations as in Chapter 4. Briefly, mRNA data are based on $n=8$ trained and $n=5$ untrained vehicle-treated animals, and $n=7$ trained and $n=5$ untrained THC-treated animals. Western blot analyses are based on $n=11$ trained animals and $n=10$ untrained animals in each treatment group. Immunohistochemical analyses in this chapter are based on a separate set of $n=7$ trained and $n=6$ untrained vehicle-treated animals, and $n=6$ trained and $n=4$ untrained THC-treated animals. A separate group of animals was used, as this analysis was conducted in the same region used for protein and mRNA expression analyses and required the preservation of morphology.

\subsection{Results}

\subsubsection{Real-Time PCR Validation}

\subsubsection{Primer Efficiencies}

Serial dilutions of purified PCR product covering a 4 log scale were amplified in duplicate, and $\mathrm{Ct}$ values plotted against the log of the dilution (Fig. 20). The slope of the trend line of these graphs was used to determine primer efficiency (Peters, Helps et al. 2004). Ideally the efficiency of primer pairs should be $100 \%$, indicating maximum assay sensitivity. With the exception of DCX, all primer efficiencies were close to $100 \%$. 

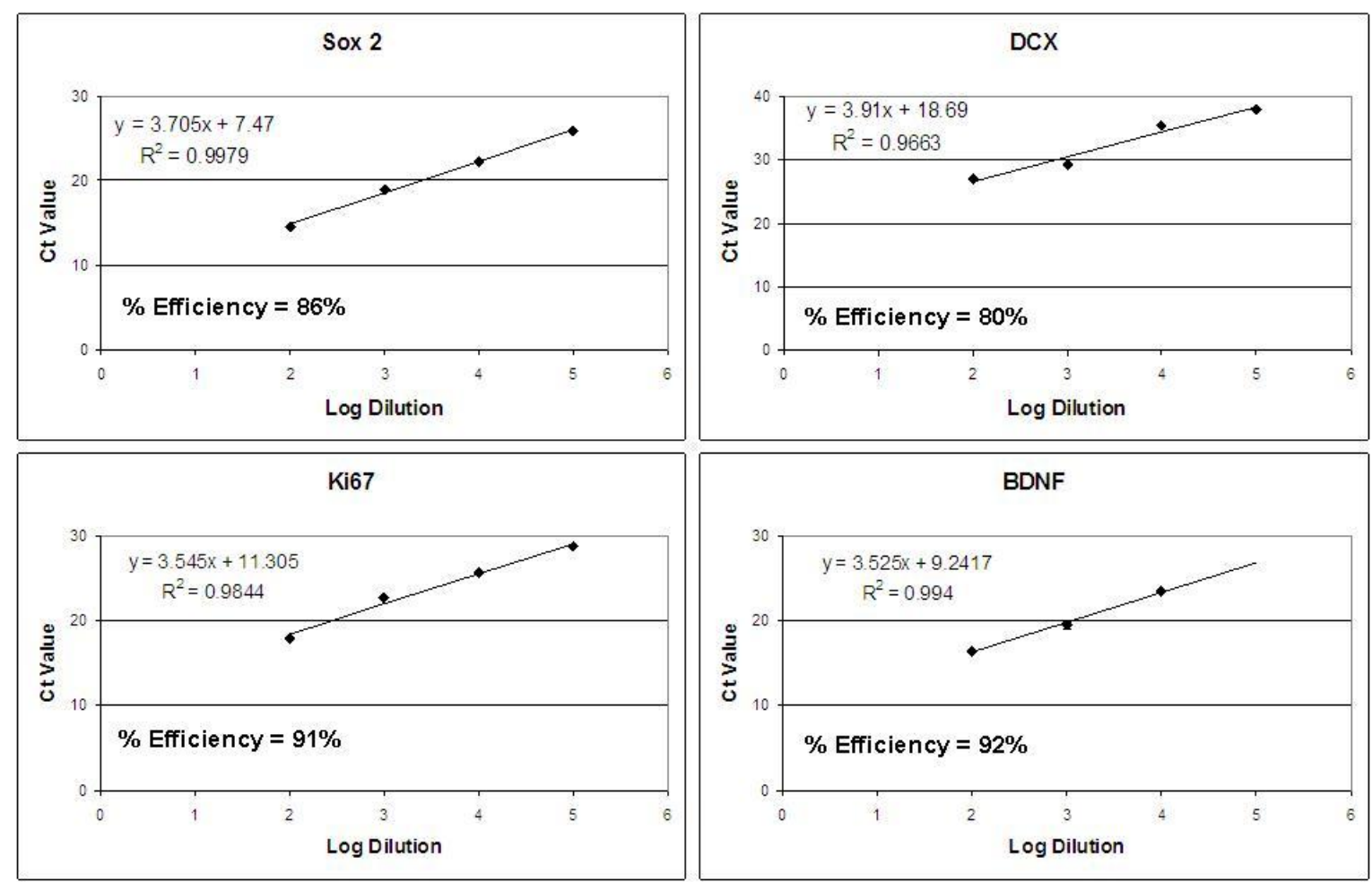

Figure 20: Efficiencies of the primers used to assess markers of neurogenesis. Purified PCR products were diluted and amplified in duplicate, and the mean $\mathrm{Ct}$ value plotted against the log of the dilution. The slope of the best fit line was used to calculate primer efficiencies (displayed on the graph). $R^{2}$ values demonstrate the fit of the trend line to the data.

\subsubsection{Melt Curve Analysis}

Following the completion of the final PCR amplification cycle (representative sample trace shown in Fig. 21A), melt curve analysis was used to determine the specificity of the PCR products. A single product is illustrated by a single peak when the rate of fluorescence change is plotted against the temperature. The temperature at which the peak occurs is determined by the base composition of the product.

A single sharp peak was observed in the melt curves for cyclophilin $A\left(84^{\circ} \mathrm{C}\right)$, Sox2 $\left(89.5^{\circ} \mathrm{C}\right), \mathrm{DCX}\left(85^{\circ} \mathrm{C}\right), \mathrm{Ki} 67\left(82.5^{\circ} \mathrm{C}\right)$ and $\operatorname{BDNF}\left(81.5^{\circ} \mathrm{C}\right)$, indicating the presence of a single PCR product. All primer sets appeared to have at least some level of primer dimer, as suggested by the presence of a small amount of product between 
$73^{\circ} \mathrm{C}$ and $86^{\circ} \mathrm{C}$ (Fig. 21B). PCR products were resolved and visualised using agarose gel electrophoresis to confirm their specificity on the basis of size (Fig. 21C). All primer sets amplified a single product of the expected size (see section 2.4). In addition, the Ki67 primer pair appeared to also amplify a small amount of off-target product of approximately $500 \mathrm{bp}$. This product was, however, scarce in comparison with the major, expected product of the PCR reaction, and its contribution to the analysis was considered to be negligible (Peters, Helps et al. 2004).
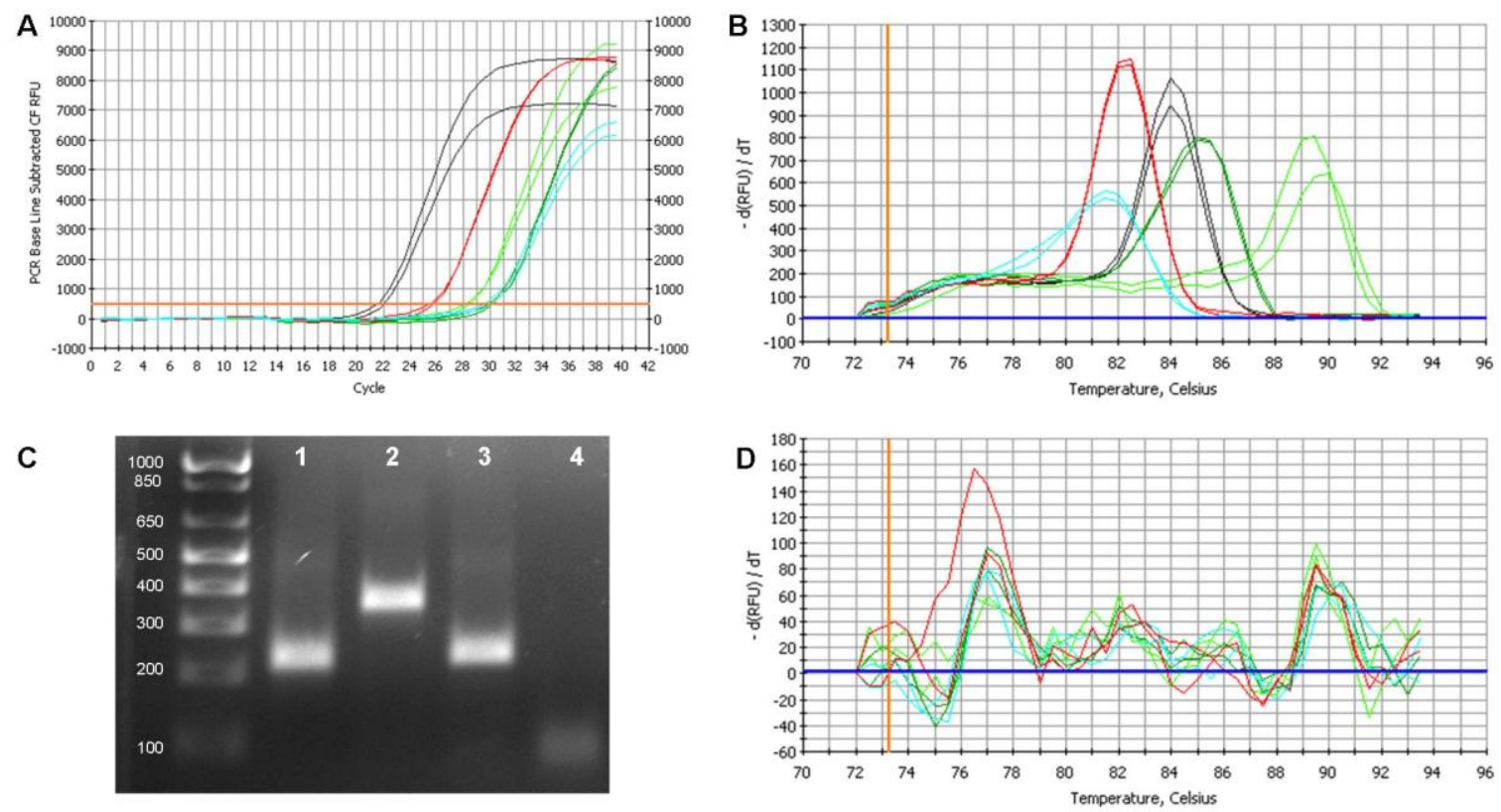

Figure 21: Representative real-time PCR data used to assess neurogenesis. (A) A representative trace image of data acquired by real-time PCR for target genes used in this chapter. All genes (cyclophilin A, black; Ki67, red; Sox2, lime; DCX, green; BDNF, teal) were analysed in duplicate to ensure the accuracy of the results. (B) Melt-curve profile analysis of these samples revealed single sharp peaks for cyclophilin $\mathrm{A}\left(84^{\circ} \mathrm{C}\right)$, Sox2 $\left(89.5^{\circ} \mathrm{C}\right)$, DCX $\left(85^{\circ} \mathrm{C}\right)$, $\mathrm{Ki} 67\left(82.5^{\circ} \mathrm{C}\right)$, BDNF $\left(81.5^{\circ} \mathrm{C}\right)$. Low levels of product were also detected at $73-86^{\circ} \mathrm{C}$ for all primer pairs. (C) Single PCR products were validated according to size using agarose gel electrophoresis (lane 1 , Sox2; lane 2, DCX; lane 3, Ki67; lane 4, BDNF). (D) Melt-curve profile analysis of negative controls (PCR reactions completed with template replaced with either $\mathrm{ddH}_{2} \mathrm{O}$ or equi-molar, non-reverse transcribed RNA) showed no specific PCR product for any of the genes analysed. 
For each primer pair, PCR amplification and melt curve analysis of negative controls detected no specific PCR product (Fig. 21D). Thus, no contaminating PCR product or genomic DNA were amplified during the PCR reaction.

\subsubsection{THC Does Not Affect Enhanced Neuronal Survival Associated with Learning in a Cohort of BrdU-Labelled Neurons}

BrdU labelling and immunohistochemistry were used to assess neuronal survival after 2 weeks (Table 6), and a significant difference between group means was detected $(p<0.001)$. There were no significant effects of THC on survival in either trained or untrained animals. Additionally, training-enhanced survival of $\mathrm{BrdU}^{+}$ neurons was seen in both vehicle-treated $(p<0.001)$ and THC-treated animals $(p<0.05)$. Furthermore, no difference in the relative distribution of labelled neurons throughout the dentate gyrus was detected between training and treatment groups (Fig. 22).

\begin{tabular}{ccc}
\hline & Trained & Untrained \\
\hline THC & $141 \pm 8$ & $103 \pm 12$ \\
Vehicle & $152 \pm 9$ & $90 \pm 7$ \\
\hline
\end{tabular}

Table 6: Number of BrdU labelled neurons surviving for 2 weeks. THC had no effect on the survival of BrdU-labelled neurons. Data are mean counts \pm SEM counts. 
a

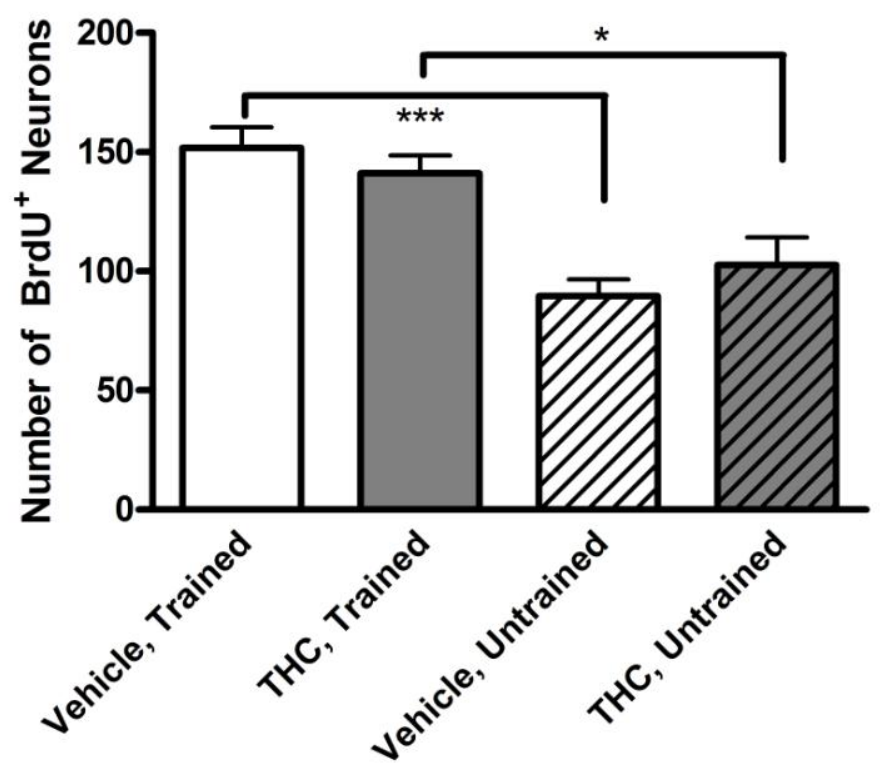

b

\section{Regional Distribution of Surviving BrdU + Cells}

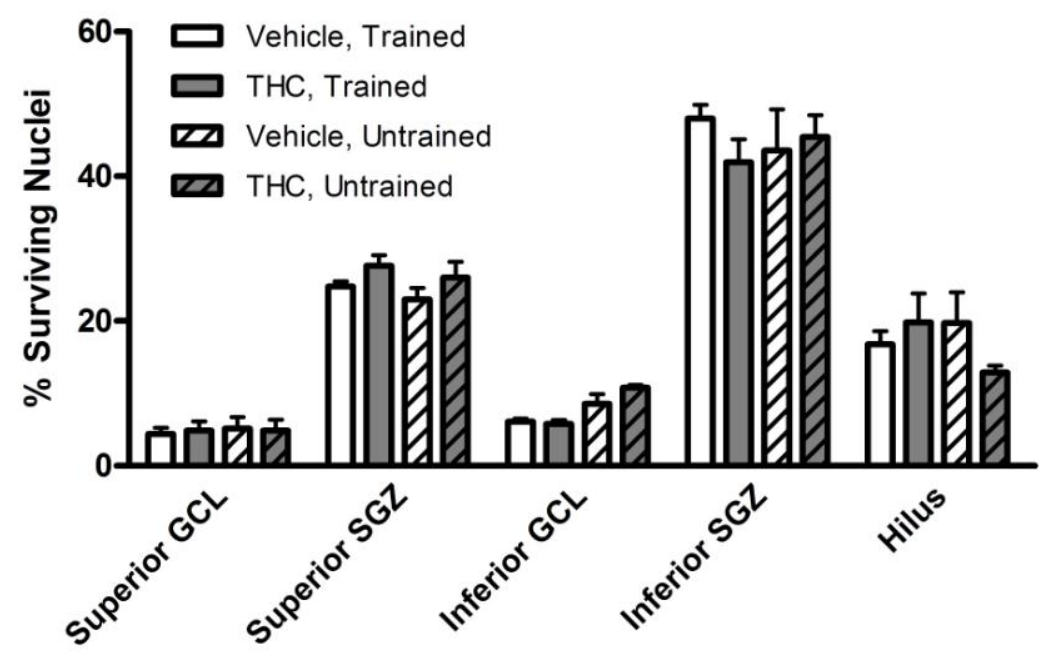

Figure 22: THC does not affect the number or localisation of surviving BrdU-labelled neurons. A) Training in the radial maze enhanced the survival of $\mathrm{BrdU}^{+}$neurons labelled 2 weeks earlier in both THC-treated and vehicle-treated animals. B) The proportion of surviving $\mathrm{BrdU}^{+}$ neurons in different regions of the dentate gyrus was not affected by either training in the radial maze or drug treatment. Data are mean \pm SEM; ${ }^{*} p<0.05,{ }^{* \star *} p<0.001$ by One-way ANOVA; SGZ, subgranular zone; GCL, granule cell layer. 


\subsubsection{Neurogenesis is Affected by Learning, and THC has Only Subtle Effects}

Mean $\Delta \mathrm{Ct}$ values for each transcript were used to compare relative transcript abundance between treatment and training groups (Table 7). For presentation purposes, $\Delta \Delta \mathrm{Ct}$ values were calculated from these data relative to vehicle-treated, untrained animals (Fig. 23).

\begin{tabular}{|c|c|c|c|c|}
\hline \multirow{2}{*}{ Target } & \multicolumn{2}{|c|}{ Trained Animals } & \multicolumn{2}{|c|}{ Untrained Animals } \\
\hline & Vehicle-Treated & THC-Treated & Vehicle-Treated & THC-Treated \\
\hline Sox2 & $\begin{array}{c}6.86 \pm 0.17 \\
(1.04 X)\end{array}$ & $\begin{array}{c}6.64 \pm 0.15 \\
(1.35 X)\end{array}$ & $6.91 \pm 0.24$ & $\begin{array}{c}7.07 \pm 0.17 \\
(0.90 X)\end{array}$ \\
\hline $\mathrm{DCX}$ & $\begin{array}{c}8.67 \pm 0.24 \\
(0.64 X)\end{array}$ & $\begin{array}{c}9.08 \pm 0.43 \\
(0.51 X)\end{array}$ & $8.03 \pm 0.31$ & $\begin{array}{c}8.11 \pm 0.29 \\
(0.95 X)\end{array}$ \\
\hline Ki67 & $\begin{array}{c}4.54 \pm 0.22 \\
\quad(0.32 X)\end{array}$ & $\begin{array}{c}5.09 \pm 0.57 \\
(0.30 X)\end{array}$ & $2.91 \pm 0.06$ & $\begin{array}{c}3.37 \pm 0.26 \\
(0.73 X)\end{array}$ \\
\hline BDNF & $\begin{array}{c}9.21 \pm 0.15 \\
(0.60 \mathrm{X})\end{array}$ & $\begin{array}{c}9.15 \pm 0.27 \\
(0.80 X)\end{array}$ & $8.48 \pm 0.26$ & $\begin{array}{c}8.82 \pm 0.28 \\
(0.79 X)\end{array}$ \\
\hline
\end{tabular}

Table 7: Relative gene expression levels between treatment and behavioural groups for neurogenic markers. Data are mean \pm SEM $\Delta$ Ct values. Fold change relative to untrained animals of the same treatment (for trained animals), or vehicle-treated animals (for untrained THC-treated animals) are shown in parentheses.

Two-way ANOVA (training $x$ transcript) indicated that training of both THC-treated $(p<0.05)$ and vehicle-treated $(p<0.001)$ animals in the radial maze reduced mRNA expression. However, significant effects of both transcript $(p<0.05)$, and an interaction (training $\mathrm{x}$ transcript, $p<0.05$ ) were also detected for both treatment groups. The detection of an "interaction" between these factors suggests that the data be interpreted with caution. The interaction effect, however, appeared to be mediated by transcript levels of Sox2 alone, for which training and drug treatment 
had no effect. When data for Sox2 were excluded from the analysis no interaction effect was detected, and reduction in mRNA levels for both THC-treated $(p<0.01)$ and vehicle-treated animals $(p<0.001)$ upon training remained statistically significant. Bonferroni post-hoc testing and one-way ANOVA confirmed that Ki67 levels were especially affected by training, and were reduced by $70 \%$ (corresponding to $1.72 \Delta \Delta \mathrm{Ct}$ units, $p<0.05$ ) and $68 \%$ (corresponding to $1.63 \Delta \Delta \mathrm{Ct}$ units, $p<0.01$ ) for THC-treated and vehicle-treated animals, respectively. No other statistically significant effects of training were observed, although training reduced the expression of DCX by $49 \%$ (corresponding to $0.97 \Delta \Delta C t$ units) in THC-treated, and $36 \%$ (corresponding to $0.64 \Delta \Delta \mathrm{Ct}$ units) in vehicle-treated animals, respectively. Training induced a $40 \%$ reduction in BDNF levels in vehicle-treated animals, greater than the $20 \%$ reduction seen in THC-treated animals, although the levels of BDNF were ultimately equivalent between THC-treated and vehicletreated animals trained in the RAM. Overall, drug treatment had no effect on transcript levels in either trained or untrained groups, and Ki67 was reduced by training in both THC-treated and vehicle-treated animals. 


\section{mRNA Expression Relative to Untrained Controls}

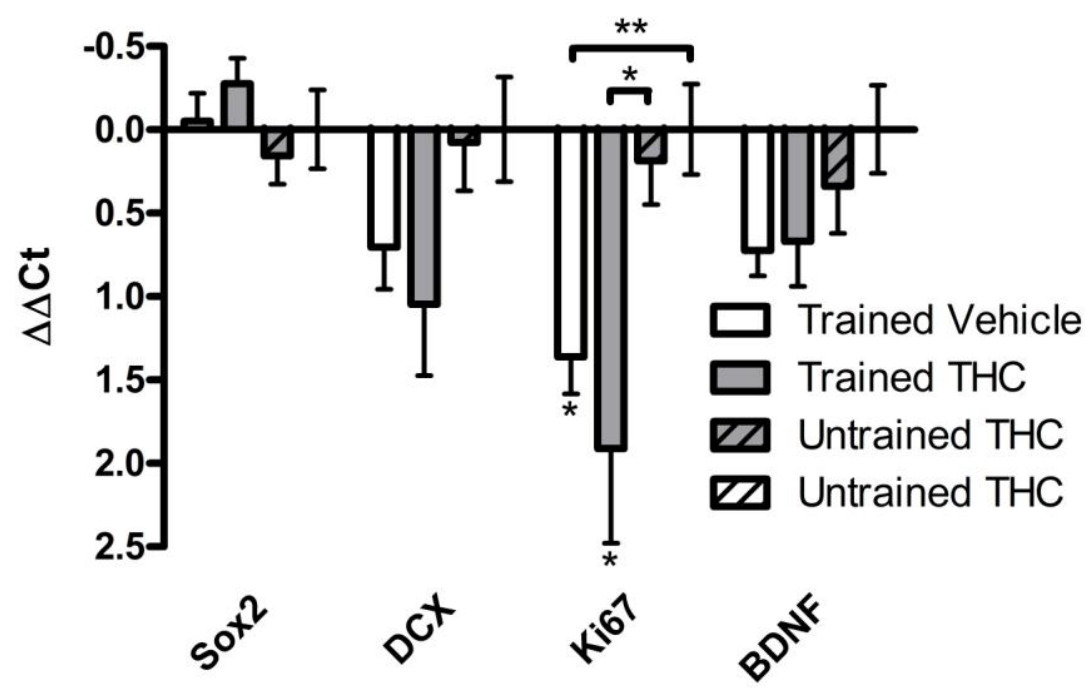

Figure 23: Training in the radial maze reduced mRNA for neurogenic markers, but was unaffected by THC. Training in the radial maze resulted in a general suppression of mRNA levels for DCX, Ki67 and BDNF; whereas, Sox2 levels were unaffected by training. Levels of Ki67 mRNA were especially reduced by training for both THC-treated and vehicle-treated animals. THCtreatment had no effect on mRNA levels for any marker. Data are mean \pm SEM $\Delta \Delta$ Ct values relative to untrained vehicle-treated animals. ${ }^{*} p<0.05,{ }^{* *} p<0.01$ by One-way ANOVA.

Hippocampal lysates were immunoblotted to determine if the trends observed in the mRNA data were corroborated by similar changes in protein expression (Fig. 24). Two-way ANOVA (training $x$ protein) indicated that training of both THCtreated and vehicle-treated animals reduced the abundance of all neurogenic marker proteins in the hippocampus ( $p<0.001$ for both). Significant reductions in the abundance of individual proteins upon training were detected with Bonferroni post-hoc testing and One-way ANOVAs. The effects of training on protein abundance were similar, but subtly different between THC-treated and vehicletreated animals. In vehicle-treated animals, training induced a $33 \%$ reduction in the abundance of Sox2 $(p<0.01)$ and a $37 \%$ reduction in the abundance of Ki67 $(p<0.05)$. Training of THC-treated animals induced a reduction in Sox2 similar to that observed in vehicle-treated animals $(32 \%, p<0.05)$, although the $27 \%$ reduction in the abundance of Ki67 was not statistically significant. Levels of PSANCAM were relatively unaffected by training in vehicle-treated animals, as only a slight (19\%) reduction was observed; whereas, training of THC-treated animals 
resulted in a much larger $44 \%$ reduction $(p<0.001)$. Modest reductions in BDNF expression upon training failed to achieve statistical significance, and no effects of THC-treatment in either trained or untrained animals were detected for any marker. 
A

Protein Abundance Relative to Untrained Controls

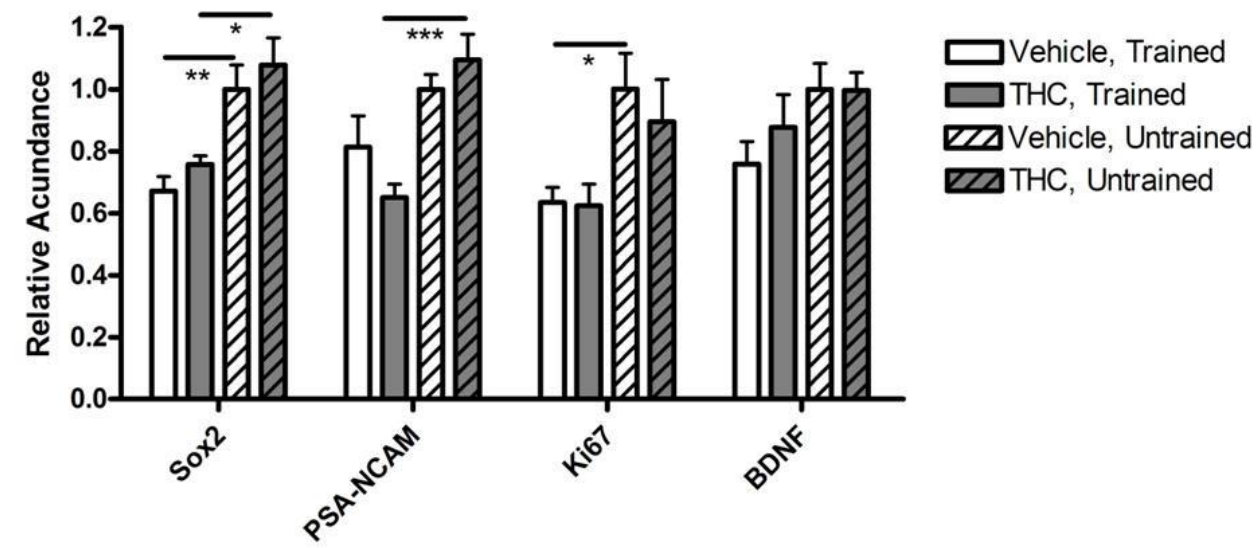

B

Trained Vehicle

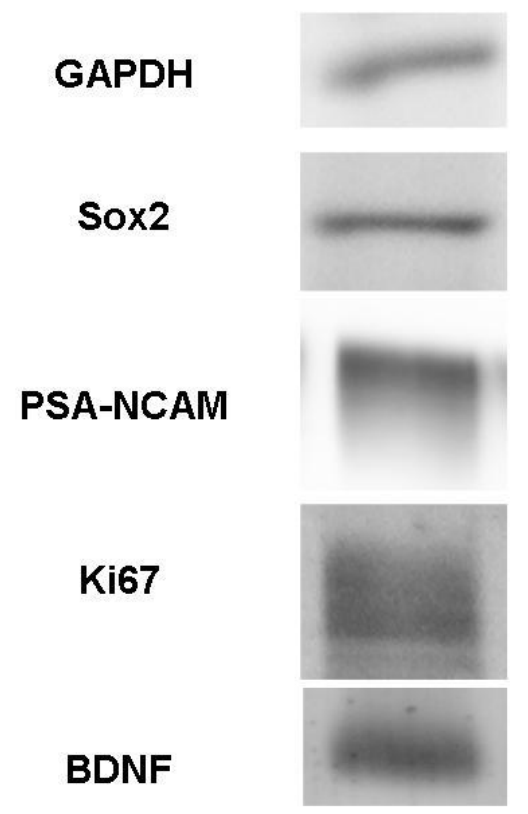

Sox2

GAPDH
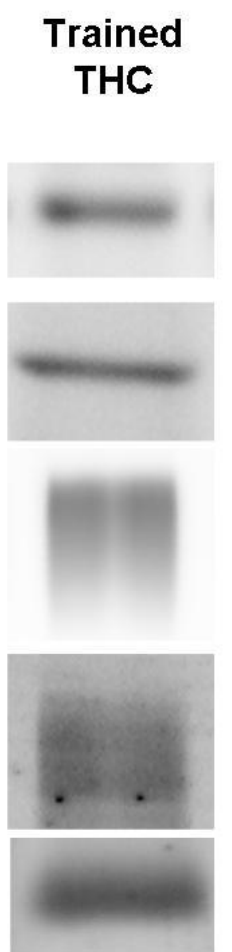

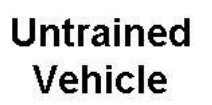

\section{Untrained THC}

Figure 24: Training in the radial maze reduces protein levels of neurogenic markers, and THC has a subtle effect. (A) Training in the radial maze reduced the abundance of neurogenic proteins in both THC-treated and vehicle-treated groups. Sox2 and Ki67 were similarly reduced by training in both treatment groups, although the reduction of Ki67 fell marginally outside significance in THC-treated animals. PSA-NCAM was reduced by training in THC-treated animals only. Data are mean \pm SEM; ${ }^{*} p<0.05,{ }^{* *} p<0.01,{ }^{* * *} p<0.001$ by One-way ANOVA. (B) Fluorescent scans of representative immunostained bands for Sox2, PSA-NCAM, Ki67 and BDNF. 


\subsection{Discussion}

Neurogenesis occurs in the adult hippocampus and is thought to contribute significantly to hippocampal plasticity by a process of pattern separation during learning and memory (Deng, Aimone et al. 2010). The survival of newborn neurons is enhanced by performing a hippocampal-dependent task (Gould, Beylin et al. 1999), such as training in the RAM. Neural progenitor cells (Aguado, Monory et al. 2005; Jiang, Zhang et al. 2005; Aguado, Palazuelos et al. 2006; Mulder, Aguado et al. 2008) and their progeny (Wolf, Bick-Sander et al. 2010) contain functional endocannabinoid signalling systems, and several aspects of neurogenesis are sensitive to cannabinoid treatment (Rueda, Navarro et al. 2002; Jin, Xie et al. 2004; Aguado, Monory et al. 2005; Jiang, Zhang et al. 2005; Aguado, Palazuelos et al. 2006; Galve-Roperh, Aguado et al. 2006; Mulder, Aguado et al. 2008). Although cannabinoids cause significant cognitive impairment (Nakamura, Dasilva et al. 1991; Lichtman, Dimen et al. 1995; Lichtman and Martin 1996; Lichtman 2000; Mishima, Egashira et al. 2001; Wise, Iredale et al. 2008) that is paralleled by significant behavioural disruption when hippocampal neurogenesis is ablated (Clelland, Choi et al. 2009; Scobie, Hall et al. 2009; Deng, Aimone et al. 2010), few studies have investigated whether behavioural disruption by cannabinoids is associated with perturbations in adult hippocampal neurogenesis. This chapter investigated the effects of THC on several aspects of hippocampal neurogenesis in the adolescent rat.

Training in a hippocampal-dependent task increases the survival of recently born neurons in the dentate gyrus (Gould, Beylin et al. 1999; Ambrogini, Cuppini et al. 2000; Epp, Spritzer et al. 2007). THC was recently shown to have no effect on neuronal survival in the hippocampus using a treatment regimen that resulted in behavioural impairment in the MWM (Wolf, Bick-Sander et al. 2010). However, the animals on which these analyses were performed were not actually trained in the MWM. Thus, while these results indicate that neuronal survival in untrained animals is not affected by THC, the effect of THC on enhanced neuron survival associated with training remains unclear. The data of this chapter indicate that in 
addition to not altering neuronal survival in untrained animals, learning-enhanced neuronal survival also appears to be unaffected by THC-treatment. Because learning may differentially enhance the survival of young neurons in the superior and inferior blades of the dentate gyrus (Ambrogini, Cuppini et al. 2000), the location of surviving neurons was recorded. No differences in the relative regional distribution of surviving neurons were observed upon THC-treatment. No difference in the survival of young neurons upon training in a spatial task does not mean that the functional properties of these neurons are equal between THCtreated and vehicle-treated groups. It remains possible that differences in the activity and architecture of these neurons exist, and that these differences may be associated with the behavioural disruption by THC observed in this study (Chapter 3). The analyses performed in this study, however, were unable to definitively resolve this possibility, although some of the data do support this hypothesis.

As previously shown (see Chapter 4), synapsin-III levels increase with training, and this effect is attenuated by THC. Synapsin-III is expressed in newborn neurons (Pieribone, Porton et al. 2002) where it is important in early outgrowth and differentiation of neural processes (Ferreira, Kao et al. 2000). Neuronal outgrowth and differentiation occurs during the first two weeks of neural development (Deng, Aimone et al. 2010). Importantly, the survival of newborn neurons in this study was followed for 2 weeks, at which time molecular analyses were conducted. Thus, attenuation of synapsin-III mRNA levels, and presumably neurite outgrowth and differentiation in THC-treated animals trained in the RAM, coincides with the second week of differentiation and development of BrdU-labelled neurons in this study, although the development of neurons born up to 1 week before training in the RAM may also have been affected by THC (Fig. 25). This suggests that the early development of this cohort of labelled neurons in trained animals is impaired by THC-treatment. Indeed, cannabinoids delay early neural development in vitro (Rueda, Navarro et al. 2002), prolonged early development of newborn neurons is associated with cognitive decline in aged animals (Nyffeler, Yee et al. 2010), and deficits in synapsin-III are associated with cognitive impairment (Kao, Li et al. 2008). Delayed early development of newborn neurons might be expected to delay later developmental events, such as the integration of young neurons into the 
hippocampal circuit and development of hyperexcitability between 2 - 4 weeks of age (Deng, Aimone et al. 2010). Indeed, the greatest behavioural differences between THC-treated and vehicle-treated groups were observed between $2-4$ weeks of training (Chapter 3), coinciding with this period. Thus, it is certainly plausible that impaired early development of the cohort of young neurons labelled with BrdU in this study leads to subsequent cognitive impairment by delaying the use of these neurons in pattern separation. This would be an interesting topic for future research.

NSCs are required for neurogenesis. NSCs can divide asymmetrically, producing another NSC to maintain the NSC pool, while also producing a transient amplifying progenitor cell that then rapidly undergoes several rounds of division to generate a pool of post-mitotic neurons (Suh, Consiglio et al. 2007). Sox2 is a transcription factor involved in maintaining the stem cell phenotype (Graham, Khudyakov et al. 2003; Ferri, Cavallaro et al. 2004; Favaro, Valotta et al. 2009), although its expression also persists during the transient amplifying progenitor stage (Liu, Namba et al. 2010). Sox2 mRNA levels were unaffected by either THC-treatment or training, and in addition, THC had no effect on Sox2 protein expression in either trained or untrained animals. Training, however, reduced the expression of Sox2 protein in both treatment groups. This reduction is likely due to a reduction in the levels of transient amplifying progenitor cells, rather than NSCs. Many Sox $2^{+}$cells are also $\mathrm{Ki} 7^{+}$, and represent transient amplifying progenitor cells (Liu, Namba et al. 2010). Reductions in Ki67 mRNA levels similar to those seen for Sox2 protein levels for both THC-treated and vehicle-treated animals were observed. A corresponding reduction was observed in the abundance of the Ki67 protein in vehicle-treated animals. Although a similar reduction was observed in THC-treated animals, this decrease was not statistically significant. Protein levels were, however, near identical between THC-treated and vehicle-treated animals in both the trained and untrained groups, suggesting that THC-treatment does not affect levels of cell proliferation, or has only minor effects. Thus, reductions in the level of proliferation in the hippocampus upon training in the RAM are unaffected by THC. 
These results are to some extent counterintuitive, since cannabinoids are generally reported to promote progenitor cell proliferation (Jin, Xie et al. 2004; Aguado, Monory et al. 2005; Jiang, Zhang et al. 2005; Aguado, Palazuelos et al. 2006; Mulder, Aguado et al. 2008), and learning is generally believed to enhance hippocampal neurogenesis (Gould, Beylin et al. 1999; Ambrogini, Cuppini et al. 2000; Epp, Spritzer et al. 2007). However, these studies have focussed on the effects of synthetic, rather than natural cannabinoids on progenitor cell proliferation, and on the effects of learning on promoting neuronal survival, respectively. Of the few studies that have used plant derived cannabinoids in vivo at doses that typically cause cognitive impairment, such as THC in this study, no effect on cellular proliferation has been reported (Kochman, dos Santos et al. 2006; Wolf, Bick-Sander et al. 2010). Very few studies have investigated the effects of learning on proliferation, rather than neuronal survival. A recent study demonstrated a reduction in the number of $\mathrm{Ki} 67^{+}$neurons in animals trained in the MWM. The reduction of proliferation upon learning may be a mechanism to counterbalance the increased survival of newborn neurons associated with learning, thus maintaining an optimal number of neurons for provision of clearer pattern separation (Epp and Galea 2009). The survival of neurons born at the beginning of training was unaffected by THC-treatment. However, because BrdUlabelling only shows that neurons of a specific age were unaffected by THCtreatment, it remains possible that the survival of young neurons of different ages may be susceptible to the effects of THC. To investigate this possibility, PSANCAM and DCX were used as markers of young neurons.

THC-treatment did not significantly affect the expression of either PSA-NCAM or DCX in untrained animals. However, training reduced the expression of PSANCAM in THC-treated animals only, and although not significant, similar expression patterns for DCX mRNA levels were seen for vehicle-treated and THCtreated, trained and untrained animals. There are several possible interpretations for these data. The progeny of progenitor cell divisions are able to commit to either the neural or glial lineage, and the endocannabinoid system has been suggested to promote astroglial differentiation (Aguado, Palazuelos et al. 2006). This could be expected to detract from the unique contribution young neurons make to 
hippocampal function (Deng, Aimone et al. 2010). While it is possible that THCtreatment resulted in a shift in the balance of neurogenesis and gliogenesis, this seems unlikely since the levels of both DCX mRNA and PSA-NCAM protein were unaffected by THC-treatment in untrained animals. In addition THC-treatment had no effect on cell proliferation (Sox2, Ki67) or the neurogenic promoter molecule BDNF, suggesting lower levels of young neuronal markers is not related to deficits in cell proliferation. Alternatively, because PSA-NCAM is involved in structural plasticity (von Bohlen und Halbach 2007), the differences in PSA-NCAM levels may be unrelated to neurogenesis. This is unlikely since the role of PSA-NCAM in structural plasticity is short-lived. Increases in PSA-NCAM levels associated with structural plasticity return to baseline levels within 24 hours of initial training, and are undetectable in whole hippocampal homogenates of the type used in this study (Murphy, Oconnell et al. 1996; Murphy and Regan 1998; Venero, Herrero et al. 2006). Finally, PSA-NCAM is most densely expressed in the SGZ of the DG where neurogenesis occurs (Nacher, Blasco-lbanez et al. 2002), and similar trends were observed using both DCX and PSA-NCAM, considered to be equivalent markers of newborn neurons (von Bohlen und Halbach 2007). Thus, it appears most likely that the differential effect of training on PSA-NCAM levels reflects differences in the number of young neurons that survive between THCtreated and vehicle-treated animals.

While the data are insufficient to categorically identify the particular subpopulation of young neurons that account for this difference, it appears likely that neurons would be either post-mitotic at the time of BrdU labelling, or born during training (Fig. 25). Enhanced young neuronal survival associated with training in a spatial task has been shown to be restricted to neurons in their second week of development (Dupret, Fabre et al. 2007; Epp, Spritzer et al. 2007). In this study animals were trained for 14 days prior to the molecular analyses. Neurons born during the second week of training would not fit within this temporal window of enhanced survival. While neurons born during the first week of training were of the correct age during the second week of training, THC had no effect on the survival of a cohort of these neurons labelled with BrdU. Thus, the neurons whose survival was enhanced by training in vehicle-treated animals were likely to be post-mitotic 
at the time of BrdU labelling. A sub-population of neurons born $6-10$ days prior to training are the most likely group to account for the differences in survival seen upon training between treatment groups. The survival of neurons of this age group has been shown to be enhanced by spatial training (Epp, Spritzer et al. 2007), and neurons born at this time would still be expressing PSA-NCAM and DCX at the time of sacrifice (von Bohlen und Halbach 2007). Finally, the temporal window for enhanced survival of neurons born before this time does not coincide with the period of training in the RAM.

It is interesting to speculate on the mechanism by which THC-treatment may elicit effects on neurogenesis as reported in this chapter. The period for enhanced survival of newborn neurons, from approximately 1-2 weeks after birth, coincides with the development of excitatory GABAergic signalling (Deng, Aimone et al. 2010). Local ambient GABA levels may inform newborn neurons of hippocampal activity, leading to their enhanced incorporation during learning ( $\mathrm{Ge}$, Goh et al. 2006). It could be expected, therefore, that a reduction in GABA release, such as that mediated by THC and other cannabinoid agonists (Heifets and Castillo 2009), would impair GABA mediated processes. Indeed, THC appeared to prevent the enhanced survival of neurons that were developing GABAergic inputs at the onset of training and THC-treatment. This effect, however, may be specific for neurons born 1 week before training, as no difference in the survival of a cohort of neurons labelled with BrdU at the beginning of training was observed. In addition to regulating neuronal survival, GABA is important for the later establishment of glutamatergic input to newborn neurons and the development of dendritic complexity (Ge, Goh et al. 2006). A reduction in presynaptic and postsynaptic markers was reported in this study (see Chapter 4), and this may be related to an impairment of synaptogenesis in newborn neurons. The attenuation of increases in synapsin-III upon training by THC supports this hypothesis, as delayed early development could be expected to delay later synaptogenesis by newborn neurons. Thus, the impairment of GABA release has the potential to explain the effects of THC on both neurogenesis and synaptic plasticity. An investigation of the role of GABA in the effects described in this thesis would be an interesting area of research. 
a

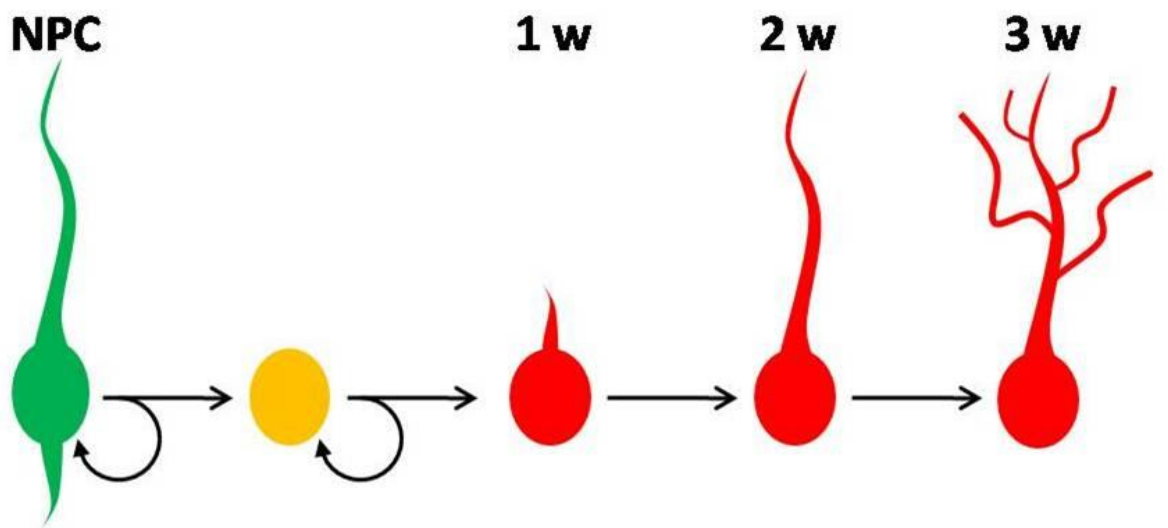

BrdU Incorp.

Sox2 / Ki67

DCX / PSA-NCAM

\section{Learning Enhanced Survival}

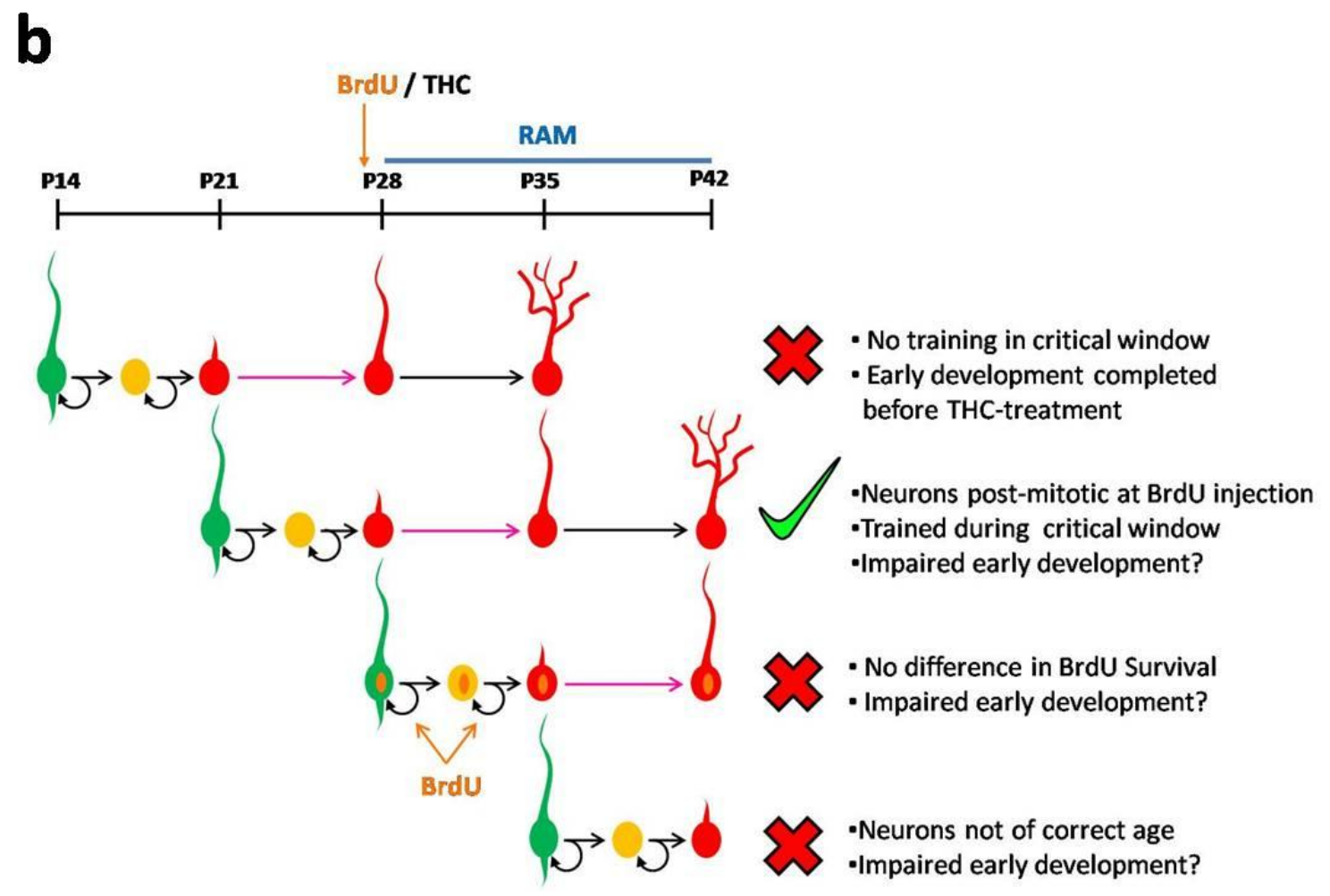


Figure 25: THC has subtle effects on hippocampal neurogenesis. A) Several stages of development lead to the production of mature granule cells in the dentate gyrus (for details, see section 1.8.2). Briefly, immature neurons are generated from successive rounds of division of transient amplifying cells that are themselves generated from the asymmetric division of a neural progenitor cell (NPC). Both progenitor cell types express the stem cell marker Sox2. In addition, transient amplifying progenitor cells express the proliferative marker Ki67, and incorporate BrdU that is then passed onto daughter cells. PSA-NCAM and DCX expression begins shortly before terminal differentiation of late transient amplifying progenitor cells and is maintained until 3 - 4 weeks of age. The number of neurons that survive to maturity is greatly enhanced by training in a hippocampal-dependent task during the second week of development (critical window), and this effect is thought to be mediated by excitatory GABAergic signalling. B) The differential effects of training on the expression of young neuronal markers in THC-treated and vehicle-treated animals is likely attributable to neurons born approximately 1 week before training begins in the radial maze. Neurons born before or after this time are unlikely to account for the difference, either because training did not coincide with the critical window of enhanced survival (pink arrows), or no difference in the survival of BrdU-labelled neurons trained in this critical window were detected. Early neurite outgrowth and development of all neurons born from one week before training onwards, however, may have been disrupted by THC-treatment. Figure adapted from (Deng, Aimone et al. 2010; Liu, Namba et al. 2010).

There are few published accounts of the effects of cannabinoids on neurogenesis during times of learning and memory, despite its important role. Previously, cellular proliferation and neuronal survival have been shown to be unaffected by THC in the absence of training (Kochman, dos Santos et al. 2006; Wolf, Bick-Sander et al. 2010). These data demonstrate that in addition to having no effect on cellular proliferation and neuronal survival in untrained animals, reduction of proliferation upon training is similarly unimpaired by THC-treatment. THC did, however, appear to reduce the survival of a subpopulation of newborn neurons that were born before, but not during training in the RAM; alternatively, the lineage commitment of new born cells may have been affected by THC but this seems less likely. Finally, the data suggest that training in the RAM enhanced the early development of newborn neurons, and that this was prevented by THC-treatment. These data are the first description of the sensitivity of neuronal survival and early development to THC during times of learning, and further investigations are warranted to better characterise these effects. 


\section{Chapter 6 General Discussion}

\subsection{Project Summary}

Of the many effects of cannabis use, the effects on learning and memory are perhaps the most worrying. This is particularly true in the case of adolescent cannabis users, since cannabis consumption is often initiated and heaviest during adolescence, a developmental period that may be especially sensitive to disruption (Spear 2000). The current literature on the effects of cannabis use on learning and memory is inadequate. Many studies have focused predominantly on the consequences of cannabis use in adult animals, with very little investigation of the molecular correlates of behavioural effects elicited by the drug. This thesis sought to address these inadequacies by investigating the cognitive deficits associated with cannabis use in adolescent animals. Furthermore, the hippocampus was assessed for neurochemical correlates of behavioural impairment. Thus, this thesis makes significant contributions to our understanding of each of these issues.

The data support the few studies that report on learning impairments in adolescents when behavioural testing is concurrent with drug treatment (see section 1.6.2). In adult animals, cannabinoids are generally believed to affect short term (working) memory, but not learning (see section 1.5.3). While the data do not specifically indicate a greater sensitivity of the adolescent period to learning deficits, as no comparison was made with adult animals, the behavioural analyses presented in this research are novel for two reasons. First, the drug treatment regimen that animals were placed on designed to model patterns of cannabis use that could be expected in human adolescents. Adolescent learning in rats was impaired 17 hours after THC-treatment, highlighting the cognitive dangers of regular cannabis use patterns outside the learning environment. Secondly, this data reports for the first time that cannabis use is associated with a disruption of the hierarchical organisation of working memory, known as 'chunking' (see 
Chapter 3). Since the second aim of this research was to investigate the effect of $\mathrm{THC}$ on molecular processes that may underlie this learning impairments, neurochemical changes from THC exposure were also analysed.

The hippocampus is a brain structure that is crucial for several forms of learning and memory. This research used a novel approach that involved both trained and untrained animals to better understand the nature of the molecular impairment operating in THC-treated animals. Subtle differences in the expression of markers of structural and functional plasticity were seen between THC-treated and vehicletreated animals trained in the RAM. When untrained animals were included in the analysis, it was discovered that changes in the levels of expression of these markers upon training were profoundly attenuated by THC-treatment. More subtle differences in neurogenesis between THC-treated and vehicle-treated groups were also detected, suggesting that neuronal survival and early development were impaired by THC-treatment whereas proliferation was not. This study is one of only a very few that have investigated the neurochemical correlates of cannabisinduced behavioural disruption.

\subsection{Strengths and Limitations of this Study}

At the beginning of this project it was expected that the neurochemical alterations in the hippocampus as a result of THC-treatment would be subtle and difficult to detect. Indeed this was the case, as when only animals trained in the RAM were compared, changes associated with THC-treatment were often not seen between THC-treated and vehicle-treated animals. When data from untrained animals were included in the analysis, however, THC had a more pronounced effect. Training resulted in specific neurochemical adaptations in vehicle-treated animals that were attenuated by THC-treatment. By including these untrained animals in the analysis it was possible to identify effects of THC-treatment that otherwise would not have been detected, and identify molecular processes that were impaired by THC, rather than molecular correlates of learning impairment. Therefore the inclusion of 
untrained animals significantly strengthened this study, and the inclusion of these animals is a major advantage over the few other studies that have investigated the neurochemical correlates of cannabis induced behavioural impairments. The inclusion of these data also likely helped overcome a potential limitation of this study by controlling for the effects of THC-treatment on adolescent development.

Adolescence in an important developmental period in which changes to the organisation and architecture of the brain precede the emergence of adult brain function (Spear 2000). In addition, learning relies on similar plasticity processes that are thought to underlie the improvement in cognitive functioning (Bailey and Kandel 1993; Moser 1999). Measuring the dynamic changes associated with learning on the background of a developmental period associated with considerable change itself (see, for example, Appendix 4) presents a potentially difficult set of circumstances for which there is no easy solution. Any neurochemical correlates of behavioural impairment associated with adolescent THC-treatment, such as those in adulthood reported by Rubino et al. (2009; 2009), may be attributable to effects of drug treatment on adolescent development in addition to any deficits in learning-induced plasticity. Alternatively, it is possible that meaningful effects of THC-treatment may be masked if the effects of drug treatment are masked by the plasticity or changes associated with learning. By controlling for the sensitivity of adolescent development to THC-treatment, this study was able to overcome this limitation. In most cases THC-treatment had no effect on the expression of markers of interest in untrained animals, demonstrating the specificity of the effects of THC for learning induced plasticity.

The use of adolescent animals, although central to the aims of this study, presented their own problems. While significant differences between the behaviour of THC-treated and control animals were detected, these corresponded to small differences in the number of correct entries in the arms of the RAM (see Chapter 3). Conventionally, to provide motivation to perform the RAM task, animals are reduced to $85 \%$ of their free-feeding weight. Such a feeding schedule is not possible in adolescent animals that are rapidly growing. Instead animals were fed 
for a 5-hour period each day, and this limit provided sufficient motivation to perform the RAM task, as well as maintain animals at a healthy weight. However, this feeding schedule was also associated with a reduced rate of learning (see Appendix 3) that has previously been linked to lower incentive values of the food rewards used to motivate behaviour (Hodges 1996). This was evident in that only about $50 \%$ of control animals achieved the learning criterion in this research (see Chapter 3), similar to the proportion of adult animals that achieved the criterion when trained under the same feeding regimen (see Appendix 3 ). The rate of learning in both adolescent and adult animals with 5 hours of access to food was much slower than conventionally motivated adult animals. These adult animals were fully trained within 5 blocks; whereas, adult and adolescent animals with only 5 hours of food access per day required 9 blocks to reach a plateau in learning. Under the training regimen used in this study, training of adolescent animals for 9 blocks was not possible as this timeframe is greater than the adolescent period in rats (Spear 2000). Reaching a plateau in learning would have been desirable because the lower error rates associated with mastery of the task would increase the sensitivity of the behavioural assay to cognitive disruption, likely resulting in an error rate better corresponding to the magnitude of learning impairment between THC-treated and vehicle-treated animals.

Modifications to the methodologies may have facilitated faster learning and an earlier learning plateau within the adolescent period. For example, a shorter feeding window may have led to more rapid learning by increasing the incentive value of the food reward. In addition, some researchers have manipulated the number of trials per day and inter-trial intervals to maximise behavioural differences between treatment groups. In doing so, the experimenter alters the amount of information presented to the individual and the requirements placed on long-term memory (Hodges 1996). Indeed, increasing the number of trials from 2 to 3 per day would have resulted in animals performing the same number of trials as had they been trained for 9 blocks required for full learning in adult animals (see Appendix 3). An alternative approach would be to use a different training apparatus more suited to the requirements of the adolescent period. For example, the MWM typically requires only half the trials of the RAM to attain a plateau in 
learning, and this can readily be achieved in the adolescent period in rats. The use of an aversive stimulus (water immersion), rather than the hunger stimulus of the RAM, also requires no pre-treatment and carries a much greater incentive value since failure to complete the task is potentially life-threatening (Hodges 1996; Paul, Magda et al. 2009). However the MWM induces more stress in animals, that could potentially affect brain function, and in addition is less adaptable to experimental requirements, such as the investigation of chunking measured in this study (Paul, Magda et al. 2009).

It is important to note that no animal model can effectively simulate all the neurochemical and behavioural attributes of human adolescence, although certain attributes of human adolescence are shared by many species. All major human brain regions are represented both structurally and functionally in the rodent brain. The development of these structures continues throughout adolescence in both species; however, the temporal scale differs significantly and is measured in days in rodents and weeks to months or even years in humans (Rice and Barone 2000; Spear 2000). THC-treatment during the 4-week adolescent period in rats resulted in a learning impairment associated specifically with an inhibition of hippocampal plasticity in this research. It is unclear whether exposure of human adolescents to THC for only 4 weeks is likely to result in lasting behavioural and neurochemical alterations that persist throughout the adolescent period, as this exposure window represents only a fraction of human adolescence. More relevant to human adolescents are the cumulative effects of cannabis use over months to years, for which an accurate animal model is not available. While no effects of THC were seen in untrained animals in this study, continued and extended exposure during a more significant proportion of human adolescence could be expected to have a far greater cumulative effect. As such, an impairment of adolescent development, in addition to an impairment of hippocampal plasticity as shown in this study, may contribute to cognitive impairment with prolonged exposure to cannabis in humans. 
Another important consideration regarding the use of rodents to model the effects of human adolescent cannabis use is the absence of social context. Adolescence is associated with increased risk taking that is augmented by peer pressure in humans. Social interaction with peers takes on significant importance during human adolescence. The desire for peer conformity can lead to antisocial behaviour such as drug taking that is often perceived as a normal and acceptable activity during adolescence (Spear 2000). By engaging in cannabis use, adolescents are more likely to adopt an unconventional lifestyle and withdraw from conventional societal expectations such as completing their education and obtaining employment (Lynskey and Hall 2000). This undoubtedly contributes to the established association between adolescent cannabis use and lower levels of educational achievement and employment (Fergusson and Boden 2008). The results of this thesis were obtained in a rodent model, free from the social contexts of adolescent cannabis use. As such, the data represent a significant learning impairment by THC as a result of direct neurochemical effects, and these may be augmented by social context in human adolescents. Importantly these effects were obtained during times of low drug burden, more accurately reflecting cognitive impairment commonly experienced by human adolescent cannabis users. However, low drug burden was assumed in this study, and it would have been interesting to determine plasma concentrations of THC during the time of behavioural testing to validate this assumption.

Some technical limitations were encountered during this research. The use of the DNA binding dye SYBR Green enabled a more rapid and cost effective assessment of several genes across four treatment groups. However, quantification using DNA binding dyes requires rigorous optimisation since DNA dyes bind to all dsDNA regardless of sequence. Good primer specificity and efficiency were therefore particularly important to ensure the high quality of the data in this research (Wong and Medrano 2005). All primer pairs generated single PCR products of the expected size. Some measurements made in this study failed to achieve statistical significance, despite apparent strong trends in the data and acceptable primer efficiencies (see Chapter 4, Chapter 5, Appendix 5). These calculated efficiencies may be inaccurate as they can be greatly affected by even 
small differences in Ct values, although this effect can be mitigated by using dilutions over a greater log range than that used in this study (Applied-Biosystems 2006). The most easily influenced factors that affect primer efficiency are primer sequence and the annealing temperature used. Greater optimisation of these parameters may have improved the power of the statistical analyses and resolved these apparent trends into meaningful changes (Wong and Medrano 2005). However, within certain limits and using comparative analyses (as in this study), less than optimal efficiencies are tolerable since the conditions are the same for all samples.

An alternative approach that would have avoided these issues would have been to use hydrolysis probes, commonly known as TaqMan chemistry. TaqMan chemistry uses traditional primers but an additional sequence specific probe is nested between them. The internal probe is conjugated to a reporter flurophore at the 3' end and an appropriate quencher at the 5' end. When annealed to the target sequence, the 5' 'exonuclease activity of DNA polymerase separates the reporter and quencher, resulting in an increase in fluorescence proportional to the amount of product produced (Wong and Medrano 2005). Because the increase in fluorescence is sequence-specific, the results are uninfluenced by any off-target priming. Furthermore, efficiency can be assured because commercially available TaqMan probes for a wide range of genes that run at $100 \%$ efficiency at $55^{\circ} \mathrm{C}$ annealing temperature are available (Applied-Biosystems 2006). The use of these probes would have provided the most sensitive assay possible and may have resulted in some of the apparent trends achieving statistical significance. The use of TaqMan chemistry, however, is associated with significant increases in cost.

Finally, some errors in sample storage and preparation were made that negatively affected the results obtained by Western blotting in initial experiments. The large number of animals required by the chosen experimental design were split into two experimental groups to ease the logistical requirements of the experiment (drug treatment, training, sample processing etc.). The analysis of behaviour and protein expression of the first group of animals, those trained in the RAM, was brought to 
completion before the treatment of the untrained animals was started. During analysis, individual polyacrylamide gels contained all samples from an individual treatment group and samples from different treatment groups were not spread evenly between gels. Results were normalised between gels and treatment groups using a protein standard loaded on all gels. Unfortunately these results had to be disregarded and the analysis repeated with fresh samples, because the antigens in the first group of animals, and the protein standard, had degraded significantly by the time attempts were made to compare the data to the second group of untrained animals. Furthermore, the inter-gel normalisation method yielded inconsistent results. When the experiments were repeated, samples were stored at $-80^{\circ} \mathrm{C}$ until all samples were available. These samples were then processed in parallel with a higher concentration of protease inhibitor and distributed evenly amongst each gel to control for differences in transfer efficiency and immunological detection. This resulted in much more consistent results, and indicated that sample age, the correct use of protease inhibitors and correct sample processing greatly influences the quality of the results obtained.

\subsection{Future Directions}

The results of this study suggest that THC has subtle effects on neurogenesis, likely to be related to an impairment of increases in neuronal survival and early development upon training seen in control animals (see Chapter 5). Several directions of future research could build substantially on these findings, beginning with a simple experiment to confirm the hypothesis that THC impairs the survival of neurons born 1 week prior to training in the RAM. This could be easily achieved by altering the timing of the BrdU injection. A similar experiment could be used to determine the effects of THC on the survival of neurons born 3 days before the beginning of training, since apoptosis is increased in neurons of this age and lower levels of apoptosis in these neurons is associated with poorer performance in the MWM (Dupret, Fabre et al. 2007). These analyses could be strengthened by including a demonstration of whether THC-treatment affects the fate-determination of new born cells in the hippocampus, because the deficits observed in trained 
THC-treated animals ultimately reflects the balance of fate specification and neuronal survival. This could be achieved using IHC and triple-fluorescent labelling to determine whether $\mathrm{BrdU}^{+}$neurons also expressed neural or glial markers. $\mathrm{A}$ result indicating that $\mathrm{THC}$ has no effect on fate determination would demonstrate that differences in young neuron number were attributable only to differences in neuronal survival. If THC was found to affect fate determination, the difference could be attributable to a combined effect on cell survival and fate specification.

The possible impairment of early development suggested by the data is more enigmatic, although it appears likely that such a delay would have a flow-on effect for later developmental events. An impairment in early neurite outgrowth and development should first be confirmed in a cohort of newborn neurons of known age using definitive immunohistochemical approaches. BrdU labelling is an inappropriate method for this assessment, because only the soma is labelled; whereas, visualisation of dendritic and axonal processes of newborn neurons is required for determining dendritic and axonal development. A retrovirus encoding green fluorescent protein (GFP) that infects only dividing cells has been developed and is well suited to this purpose, since constitutively and stably expressed GFP fills the dendritic and axonal processes as well as the soma (van Praag, Schinder et al. 2002). GFP labelling would allow the study of the effects of THC on processes of neurite outgrowth, dendritic arborisation and reception of synaptic input that all lead to the functional integration of newborn neurons into hippocampal circuits (Deng, Aimone et al. 2010).

The impact of any impairment of structural development of newborn neurons by THC would be likely to mediate behavioural disruption through changes in the unique contribution of newborn neurons to hippocampal function. Newborn neurons reach a state of hyperexcitability by 4 weeks of development that is related to their preferential incorporation into circuits supporting learning and memory (Deng, Aimone et al. 2010). The GFP-retrovirus approach is also well suited to assessing the development of hyperplasticity. Using fluorescence microscopy, cohorts of newborn neurons of a known age could be identified using 
GFP as a marker and electrophysiological measurements could be taken from the neurons (van Praag, Schinder et al. 2002; Ge, Yang et al. 2007). The preferential recruitment of newborn neurons into circuits supporting specific memories has already been studied using IHC (Ramirez-Amaya, Marrone et al. 2006; Kee, Teixeira et al. 2007; Tashiro, Makino et al. 2007; Deng, Aimone et al. 2010). Immediate early gene (IEG) expression, such as c-Fos and Arc, is tightly coupled to neuronal activity and can be used to identify individual neurons that are activated during memory retrieval. The effect of THC on this preferential incorporation could be measured by staining for IEGs in BrdU-labelled neurons of different ages immediately following training.

The hippocampus consists of 3D subfields that change rapidly in space and interact with each other via long and defined fibre tracts (see Section 1.6.1). Many of the markers used in the present study are restricted to specific regions of the hippocampus. For example, neurogenic markers are generally restricted to the dentate gyrus; whereas, others such as pre and postsynaptic markers are more widely spread between the various hippocampal regions. This study reports changes in the expression of these genes and proteins using an approach based on a homogenate of all of these subfields in the dorsal hippocampus. As such, regional topography was lost, and changes may not have been detected if they were small in size and associated with a specific region or regions. Alternatively, in the case of broad changes in expression, it is unclear with what region or regions the change is associated. This could result in valuable information being lost, such as the identification of drug effects in specific hippocampal fibre pathways. Thus, the structural heterogeneity of the hippocampus is a significant barrier to the molecular analyses of this study. By preserving the topology during sample collection and analysis it is possible to overcome some of these limitations.

Microdissection and regional analysis of the hippocampus is a promising alternative to the use of whole hippocampal homogenates. Microdissection requires precision and is commonly achieved from mounted sections using a sterile scalpel and needle under a dissection microscope. This technique has 
previously been used successfully to isolate the CA subfields and the dentate gyrus of the hippocampus (Ginsberg and Che 2004). Recent technological advancements allow this process to be automated, removing experimental error and allowing for a consistent and accurate dissection of brain regions. Of these advances, laser capture microscopy (LCM) is perhaps best suited to this study, as both RNA and protein are preserved in the dissected samples for later analyses (Walch, Specht et al. 2001). With LCM, specific regions of a specimen are dissected by adhesion to a thermoplastic polymer following precise activation of the desired area with a near infrared laser. The region can then be accurately removed from the surrounding tissue for mRNA and protein analysis (Simone, Bonner et al. 1998; Walch, Specht et al. 2001). However, the microdissection of tissue from thin histological sections results in the collection of relatively few cells. This can be overcome in the case of mRNA analysis by RNA amplification with T7 polymerase (Luo, Salunga et al. 1999); however it is uncertain whether enough protein could be collected to allow protein abundance studies. Quantitative $\mathrm{IHC}$ is an alternative method for measuring regional protein abundance and has previously been used to detect increases in synapsin-I in specific hippocampal subfields (Vaynman, Ying et al. 2004). Analyses such as these would help in determining the effects of THC-treatment on specific regions of the hippocampus, or rather to identify them as hippocampal-wide effects.

Finally this study could be replicated using adult animals, thus allowing the determination of whether the effects of THC on behavioural and molecular processes reported in this thesis are specific to the adolescent period. This research represents the first evidence that the cognitive process of chunking is impaired by THC in animals of any age, while the impairment of hippocampal plasticity by THC treatment during adolescence reported in this study appears to persist into the adult period (Rubino, Vigano et al. 2008; Rubino, Realini et al. 2009; Rubino, Realini et al. 2009). Although adolescents do appear more sensitive to the behavioural effects of cannabinoids than adult animals (Spear 2000), the absence of adult animals in this study precludes any conclusions on the basis of developmental age. 


\subsection{Final Conclusions}

This study sought to determine the effects of THC on adolescent learning and memory, as well as to identify the molecular mechanisms through which any impairment was operating. Adolescent learning was impaired by THC, and specific neurochemical changes were observed in the hippocampus. A significant strength of this research was that it showed that the higher cognitive function of chunking, as well as hippocampal plasticity in response to learning, were both affected by THC during adolescence. Thus, the study identified definitive cognitive and molecular processes that were impaired by THC. However because no comparison was made with adult animals in this study, it remains unclear whether these impairments represent a greater sensitivity of adolescents to THC, or rather whether these impairments operate in animals of all ages. Thus, this research sets a framework for future experiments designed to characterise the mechanisms of cannabis-induced learning and memory impairment. 


\section{Chapter 7 Appendices}

\section{Appendix $1 \quad$ Reagent List}

- $10 \times$ Tris Buffered Saline (TBS)

$$
\begin{aligned}
& \text { ○ } 60.5 \mathrm{~g} \mathrm{Tris-} \mathrm{HCl} \\
& \circ \quad 87.5 \mathrm{~g} \mathrm{NaCl}
\end{aligned}
$$

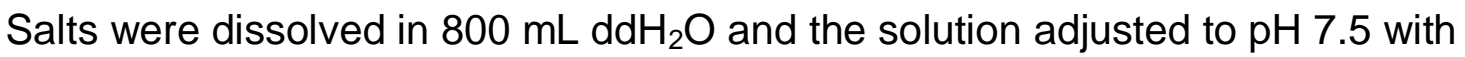
$\mathrm{HCl}$. The solution was then made up to $1 \mathrm{~L}$ with $\mathrm{ddH}_{2} \mathrm{O}$. TBS was split into two 500 $\mathrm{mL}$ containers and autoclaved. 1 x TBS was prepared from this stock.

- TBS-tween (TBST)

○ $1 \mathrm{~L} 1 \times \mathrm{TBS}$

○ $1 \mathrm{~mL}$ Tween-20

Mix constituents.

- $10 \times$ Phosphate Buffered Saline (PBS)

- $80 \mathrm{~g} \mathrm{NaCl}$

- $14.2 \mathrm{~g} \mathrm{Na}_{2} \mathrm{HPO}_{4}$ (anhydrous)

- $2 \mathrm{~g} \mathrm{KCl}$

- $2.4 \mathrm{~g} \mathrm{KH}_{2} \mathrm{PO}_{4}$ 


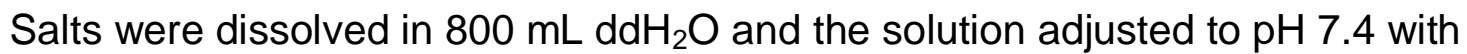
$\mathrm{HCl}$. The solution was then made up to $1 \mathrm{~L}$ with $\mathrm{ddH}_{2} \mathrm{O}$. PBS was split into two 500 $\mathrm{mL}$ containers and autoclaved. $1 \times$ PBS $(0.15 \mathrm{M})$ was prepared from this stock.

- 5 x Western Running Buffer

○ $15 \mathrm{~g}$ Tris- $\mathrm{HCl}$

- $72 \mathrm{~g}$ Glycine

- $5 \mathrm{~g} \mathrm{SDS}$

Salts were dissolved in $1 \mathrm{LddH} \mathrm{H}_{2} \mathrm{O}$ without $\mathrm{pH}$ adjustment. $1 \mathrm{x}$ running buffer was prepared from this stock.

- Western Transfer Buffer

- $3.03 \mathrm{~g}$ Tris- $\mathrm{HCl}$

○ $14.4 \mathrm{~g}$ Glycine

$\circ 0.38 \mathrm{~g}$ SDS (when required for the transfer of high molecular weight proteins)

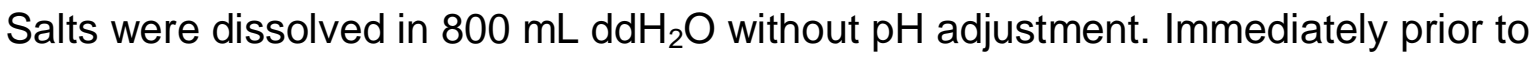
use $200 \mathrm{~mL}$ methanol was added to this solution. Transfer buffer was made fresh as required.

- 5 x Reducing Buffer

○ $1 \mathrm{~mL} 1.5 \mathrm{M}$ Tris-HCl pH 6.8

- $5 \mathrm{~mL} 10 \%$ SDS 
○ $3.2 \mathrm{~mL} 80 \%$ Glycerol

- $0.8 \mathrm{~mL} 1 \%$ Bromophenol Blue

- $\quad$ DAB solution $(0.5 \mathrm{mg} / \mathrm{mL})$

○ $5 \mathrm{mg} \mathrm{DAB}$

○ $10 \mathrm{~mL} 1 \times$ PBS

DAB was dissolved in $1 \times$ PBS by vortexing, separated into $1.5 \mathrm{~mL}$ aliquots and frozen at $-20^{\circ} \mathrm{C}$. Immediately prior to use aliquots were thawed and $1.5 \mu \mathrm{L}$ of $30 \%$ $\mathrm{H}_{2} \mathrm{O}_{2}$ was added to a final concentration of $0.03 \%$. This solution was then applied to histological sections to reveal HRP conjugates.

- $4 \%$ Paraformaldehyde (PFA)

- $20 \mathrm{~g}$ Paraformaldehyde

○ $480 \mathrm{~mL}$ PBS

Paraformaldehyde was dissolved in $400 \mathrm{~mL} 1$ x PBS, made to $\mathrm{pH} 12.0$ by addition of $\mathrm{NaOH}$, and dissolved by heating and stirring. Once dissolved the solution was adjusted to $\mathrm{pH} 7.4$ with $\mathrm{HCl}$ and made up to $500 \mathrm{~mL}$ with $1 \times$ PBS.

- RIPA Buffer

○ 606 mg Tris-HCl

○ $877 \mathrm{mg} \mathrm{NaCl}$

○ $37.22 \mathrm{mg} \mathrm{Na}$ EDTA 
- $1 \mathrm{~mL}$ Triton-X 100

- $1 \mathrm{~g}$ Sodium Deoxycholate

○ $100 \mathrm{mg}$ SDS

Mix constituents to $100 \mathrm{~mL}$ with $\mathrm{ddH}_{2} \mathrm{O}, \mathrm{pH}$ to 7.4 .

- Acid Ethanol

○ $100 \mathrm{~mL} 70 \%$ Ethanol

○ $0.25 \mathrm{~mL}$ concentrated $\mathrm{HCl}$

Mix constituents.

- $10 \%$ acrylamide resolving gel (enough for 2 gels)

○ $8.2 \mathrm{~mL} \mathrm{dd} \mathrm{H}_{2} \mathrm{O}$

○ $5 \mathrm{~mL} 1.5 \mathrm{M}$ Tris- $\mathrm{HCl}, \mathrm{pH} 8.8$

- $200 \mu \mathrm{L} 10 \% \mathrm{w} / \mathrm{v}$ SDS

○ $6.6 \mathrm{~mL} 30 \%$ Acrylamide/Bis

○ $100 \mu \mathrm{L} 10 \% \mathrm{w} / \mathrm{v}$ APS

○ 10 $\mu \mathrm{L}$ TEMED

Gently mix and pour into casting module. Layer $100 \%$ isopropanol on top while gel dries, but remove prior to layering with stacking gel. 
- $4 \%$ acrylamide stacking gel (enough for 4 gels)

○ $6.1 \mathrm{~mL} \mathrm{ddH} \mathrm{H}_{2} \mathrm{O}$

○ $2.5 \mathrm{~mL} 0.5 \mathrm{M}$ Tris- $\mathrm{HCl}, \mathrm{pH} 6.8$

○ $100 \mu \mathrm{L} 10 \% \mathrm{w} / \mathrm{v}$ SDS

- $1.3 \mathrm{~mL} 30 \%$ Acrylamide/Bis

- $50 \mu \mathrm{L} 10 \% \mathrm{w} / \mathrm{v}$ APS

- $10 \mu \mathrm{L}$ TEMED

Gently mix and pour on top of resolving gel, then insert comb and allow to dry. 


\section{Appendix $2 \quad$ Hematoxylin staining of brain sections following $D A B$ staining}

Hematoxylin was used as a nuclear stain in this research project as it was relatively insensitive to the $2 \mathrm{~N} \mathrm{HCl}$ denaturation required for the detection of the BrdU epitope. In contrast, Nissl (thionin) staining was almost undetectable following the $2 \mathrm{~N} \mathrm{HCl}$ denaturation. To stain cell nuclei with hematoxylin sections:

- Wash sections $2 \times 2$ min in $\mathrm{ddH}_{2} \mathrm{O}$

- Immerse sections in Harris type Hematoxylin stain for $30 \mathrm{~s}$

- Briefly rinse sections in 2 changes of $\mathrm{ddH}_{2} \mathrm{O}$

- Wash sections in tap water for 5 min

- Destain sections for $1 \mathrm{~min}$ in acid ethanol

- Rinse sections $2 \times 1$ min in tap water

- Rinse sections 2 min ddH $\mathrm{H}_{2} \mathrm{O}$

- Dehydrate sections

- $1 \times 2$ min in each of $70,80,90,100 \%$ Ethanol

- $2 \times 10$ min in $100 \%$ Xylene

- Remove excess Xylene from sections and mount using DPX mounting medium 


\section{Appendix 3 Considerations of the Effect of Animal Treatment on Learning}

The RAM relies on motivating animal behaviour through hunger. This motivation is commonly achieved by restricting food intake to maintain animals at $85 \%$ of their free-feeding weight. This method is unsuitable for adolescent rats that are rapidly growing. An alternative is to allow free access to food for a limited period each day. To determine whether the period of access affected motivation to collect rewards in the RAM, groups of adolescent animals were allowed either free access to food, or their access was restricted to a 3, 4, 5 or 6 hour period per day. Following 1 week of their respective feeding schedules, animals were placed in the RAM in which every arm was rewarded for $10 \mathrm{~min}$, and the number of rewards collected over 3 sessions ( 1 session per day) was recorded. A similar number of rewards were consumed by animals in each of the restricted feeding groups, and all restricted feeding groups consumed more rewards than animals with free access to food (Fig. 26A). Thus, this study used a 5-hour feeding window which provided sufficient motivation in the RAM while providing maximum access to food. It also fit well with the logistical requirements of the experiment.

To ensure that the restriction of animals' access to food did not cause unacceptable suffering to the animals in this experiment, the weight gain of a small number of both THC-treated and vehicle-treated animals was followed over the experimental timeframe. While THC-treatment reduced the rate of weight gain, an animal care technician determined that all animals were healthy. Figure 26B shows the weight gain of all animals that were subsequently used in behavioural analyses. The rate of weight gain was reduced in THC-treated animals $(p<0.001$, Two-way ANOVA), and this was especially evident from approximately 2 weeks of treatment.

To determine the effect of using a restricted access (rather than a reduced weight) motivational approach, these methods were directly compared in adult animals. 
Animals in the weight-reduced group learnt the maze more rapidly than those in the restricted access group using measures of both cumulative percent that achieved the learning criterion (see Chapter 3 for details) and the overall group accuracy (Fig. 26C,D). Differences in the rates of learning presumably represent a reduction in motivation in the restricted access group, and similar performances using this method were seen in both adolescent and adult animals. It could be expected that reduced motivation and slower learning would result in a less sensitive assay for learning impairment. Thus, the true magnitude of the cognitive impairment by THC detected in this research (see Chapter 3) may in fact be larger than that described in this research.
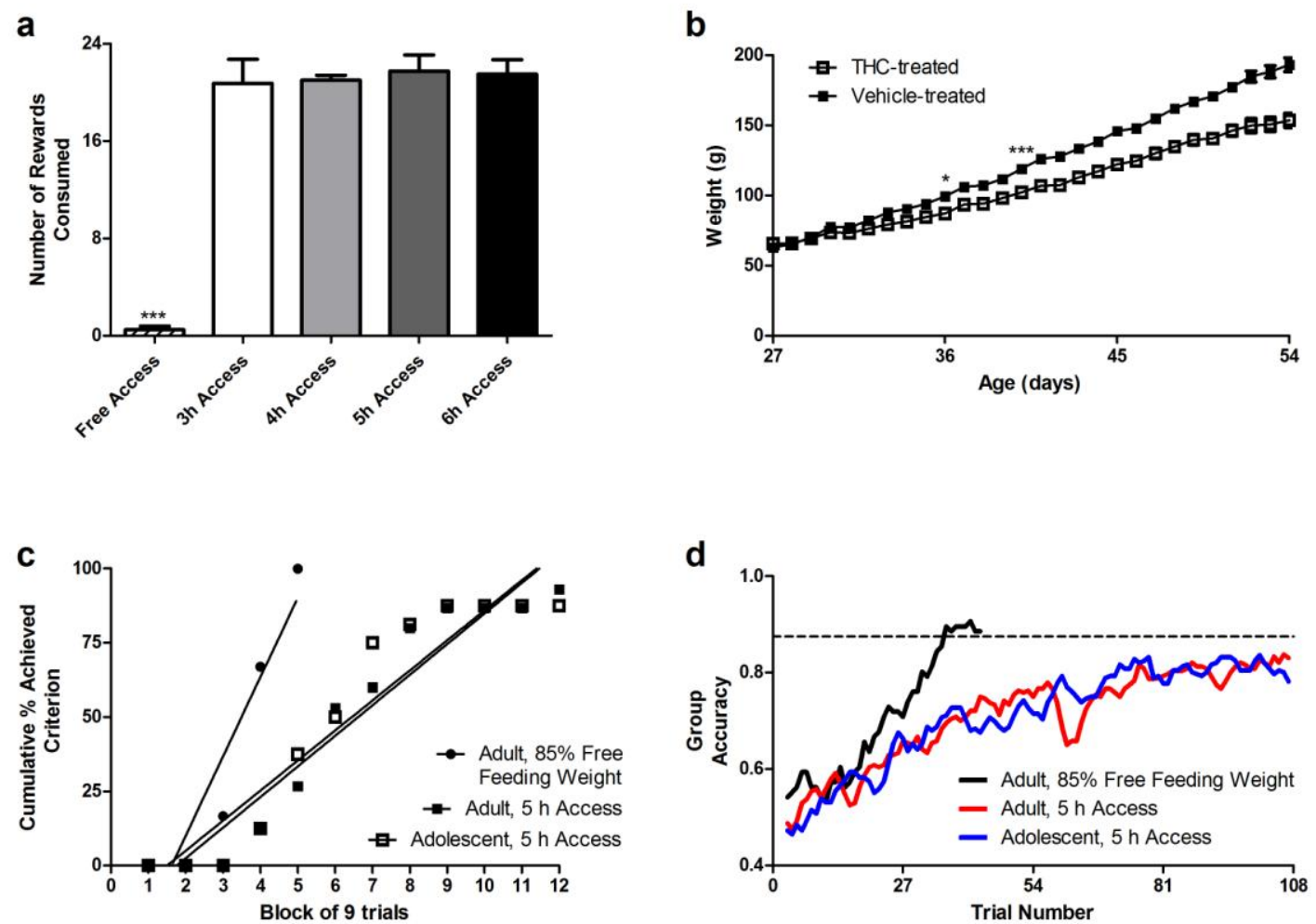

Figure 26: Considerations of the effects of animal treatment on learning. A) Food restriction was required to motivate adolescent animals to retrieve rewards in the RAM, but shorter restriction did not affect motivation; $n=4$ animals in each group; asterisk indicate fewer rewards collected than any other group. B) THC impaired the rate of weight gain in adolescent animals; $n=23$ animals for both treatment groups, asterisks indicate the first significant differences seen. (C, D) Adolescents and adults with restricted food access learnt at similar rates, but reduction of adult baseline weight resulted in faster learning as measured by achievement of the learning criterion $(p<0.01, C)$ and overall group accuracy $(p<0.001, D) ; n=6$ adult weight reduced, 16 adult food restricted, and 15 adolescent food restricted animals. Data are mean $(A, B, D) \pm$ SEM $(A, B) ;{ }^{*} p<0.05,{ }^{* *} p<0.001$. 


\section{Appendix 4 Developmental Gene Expression}

Adolescence is a neurodevelopmental period in which significant synaptic pruning underlies the emergence of the adult neural architecture (Spear 2000; Adriani and Laviola 2004). Preliminary studies were conducted to characterise this process in preparation for later investigations of whether behavioural deficits in adolescence following in utero and neonatal cannabis exposure (Schneider 2009) were associated with changes in structural and functional plasticity, as reported for adolescent exposure in this thesis (see Chapter 4). However, these experiments were later abandoned to focus on completing other experiments already in progress.

Data were collected from male rats that were $7,14,21$ or 28 days old, and from adults weighing more than $300 \mathrm{~g}$. Animals up to 28 days old were maintained with their mothers, and adult animals were housed in pairs with food and water available at all times. Animals aged 7 and 14 days were sacrificed by rapid decapitation without carbon dioxide asphyxiation, while all other older animals were asphyxiated before decapitation. Statistical analysis were conducted using the number of litters analysed as the base value for ' $n$ ' $(n=1)$ in animals aged up to 28 days. This was done because it has been shown that in developmental studies conducted using multiparous species such as the rat, treatment of each individual within the litter as an independent observation can triple the likelihood of achieving the alpha level. This is because individuals within a litter are more likely to be similar to each other than to individuals of another litter (Holson and Pearce 1992). For each litter, hippocampal tissue was collected from three males and then combined to make a single sample. This sample was then analysed as a discrete observation. For adult animals the base value for ' $n$ ' was designated as an individual animal. 


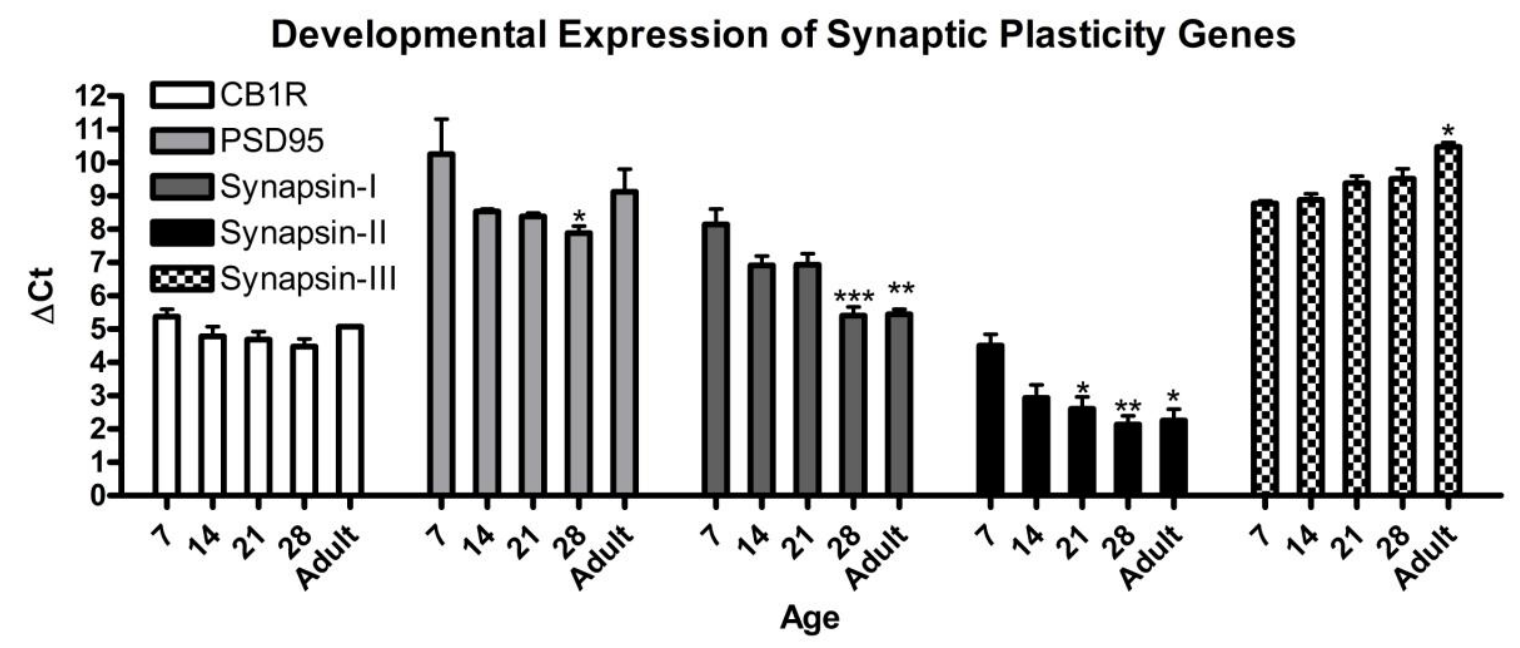

Figure 27: The expression of transcripts involved in synaptic plasticity is developmentally regulated. In most cases expression appears to peak at 4 weeks of age, coinciding with the beginning of the adolescent period, before declining into adulthood. Data are mean \pm SEM $\triangle \mathrm{Ct}$ values, significant changes in expression compared to $P 7$ levels are indicated. ${ }^{*} p<0.05,{ }^{* *} p<0.01$, ${ }^{* * *} p<0.001$.

Significant changes in expression during development were detected for PSD95 ( $p=0.022)$, synapsin-I $(p=0.001)$, synapsin-II $(p=0.011)$ and synapsin-III $(0.015)$, but not CB1R ( $p=0.21$ ) (Fig. 27). PSD95 and synapsin-I had increased in expression over levels observed in P7 animals by 4 weeks of age; while, synapsinII had increased by 3 weeks of age. These differences were less pronounced in adulthood. While not statistically significant, this general trend was also observed for CB1R; whereas, synapsin-III levels decreased during development, dropping significantly below levels observed in P7 animals by adulthood (Table 8). These data are largely supported by previous studies that have shown a decline in synapsin-III mRNA levels in vitro (Ferreira, Kao et al. 2000), a peaking of CB1R levels during adolescence (Spear 2000), and the development of synaptic connections during neonatal development into adolescence (Zurmohle, Herms et al. 1996; Lu, Rong et al. 2000). 


\begin{tabular}{|c|c|c|c|c|c|}
\hline \multirow{2}{*}{ Target } & \multicolumn{5}{|c|}{ Age } \\
\hline & $7(n=2)$ & $14(n=3)$ & $21(n=3)$ & $28(n=4)$ & Adult $(n=2)$ \\
\hline \multirow[t]{2}{*}{ CB1R } & $5.38 \pm 0.22$ & $4.78 \pm 0.29$ & $4.68 \pm 0.25$ & $4.48 \pm 0.23$ & $5.08 \pm 0.02$ \\
\hline & & $(1.52 X)$ & $(1.62 X)$ & $(1.87 X)$ & $(1.23 X)$ \\
\hline \multirow[t]{2}{*}{ PSD95 } & $10.25 \pm 1.05$ & $8.53 \pm 0.07$ & $8.38 \pm 0.10$ & $7.89 \pm 0.21$ & $9.13 \pm 0.68$ \\
\hline & & (3.29X) & $(3.66 X)$ & $(5.13 X)$ & $(2.17 X)$ \\
\hline \multirow[t]{2}{*}{ Synapsin-I } & $8.15 \pm 0.45$ & $6.92 \pm 0.27$ & $6.93 \pm 0.33$ & $5.40 \pm 0.27$ & $5.45 \pm 0.15$ \\
\hline & & $(2.35 X)$ & $(2.33 X)$ & $(6.73 X)$ & $(6.50 X)$ \\
\hline \multirow[t]{2}{*}{ Synapsin-II } & $4.5 \pm 0.35$ & $2.93 \pm 0.39$ & $2.60 \pm 0.37$ & $2.14 \pm 0.26$ & $2.25 \pm 0.35$ \\
\hline & & $(2.97 X)$ & $(3.73 X)$ & $(5.13 X)$ & $(4.76 X)$ \\
\hline \multirow[t]{2}{*}{ Synapsin-III } & $8.78 \pm 0.08$ & $8.88 \pm 0.19$ & $9.38 \pm 0.21$ & $9.51 \pm 0.30$ & $10.48 \pm 0.13$ \\
\hline & & $(0.93 X)$ & $(0.66 X)$ & $(0.60 X)$ & $(0.31 X)$ \\
\hline
\end{tabular}

Table 8:Relative gene expression levels between developmental stages for plasticity

markers. Data are mean $\pm \Delta$ Ct values, $\mathrm{n}$ as indicated. Fold change in expression relative to P7 animals is shown in parentheses. 


\section{Appendix $5 \quad$ THC and the Renin-Angiotensin System}

The renin-angiotensin system (RAS) is perhaps best known for its role in the regulation of blood pressure, although a role in modulating learning and memory processes is emerging. All angiotensin peptides originate from the pro-hormone angiotensinogen, from which the angiotensin peptides (Ang) I and Angll are cleaved. Angl, containing all the peptide sequences necessary for signalling by the RAS but possessing little biological activity, is cleaved from angiotensinogen by the aspartyl protease renin. Conversion of Angl to Angll by the angiotensin converting enzyme (ACE) results in the first bioactive form of angiotensin, and Angll acts on both the angiotensin II receptor (AT2R)1 and AT2R2. Angll can be further converted to AngIV and Ang1-7 that have retained but modified bioactivity, and act on distinct receptor classes. Components of the RAS required for peptide synthesis and signalling are widely expressed on neurons and glia in the brain where the angiotensins act as neuropeptides. Angll, when delivered exogenously, increases the firing rate of neurons in several brain regions including the hippocampus and impairs performance in various learning and memory tasks. It appears likely that these impairments are associated with the suppressive effects of Angll on LTP induction, although opposite effects of the angiotensin peptides have also been described (Hellner, Walther et al. 2005; Kerr, Bevilaqua et al. 2005; von Bohlen und Halbach 2005; Braszko, Walesiuk et al. 2006; Akhavan, Emami-Abarghoie et al. 2008).

Because of the emerging role of the RAS in learning and memory processes, the effect of THC-treatment on the expression of components of the RAS in trained and untrained animals was assessed in the present study. Messenger RNA for ACE, AT2R2 and angiotensinogen in the hippocampus was measured in the same samples using the same methods for which markers of structural and functional plasticity (Chapter 4), as well as neurogenesis (chapter 5), were assessed.

Several primer pairs for AT2R1 were tested; however, all pairs generated multiple PCR products and were thus unsuitable for mRNA quantification. Primer pairs for 
ACE, AT2R2 and angiotensinogen, however, had efficiencies close to $100 \%$ (Fig. 28) and generated single PCR products (Fig. 29B, C).
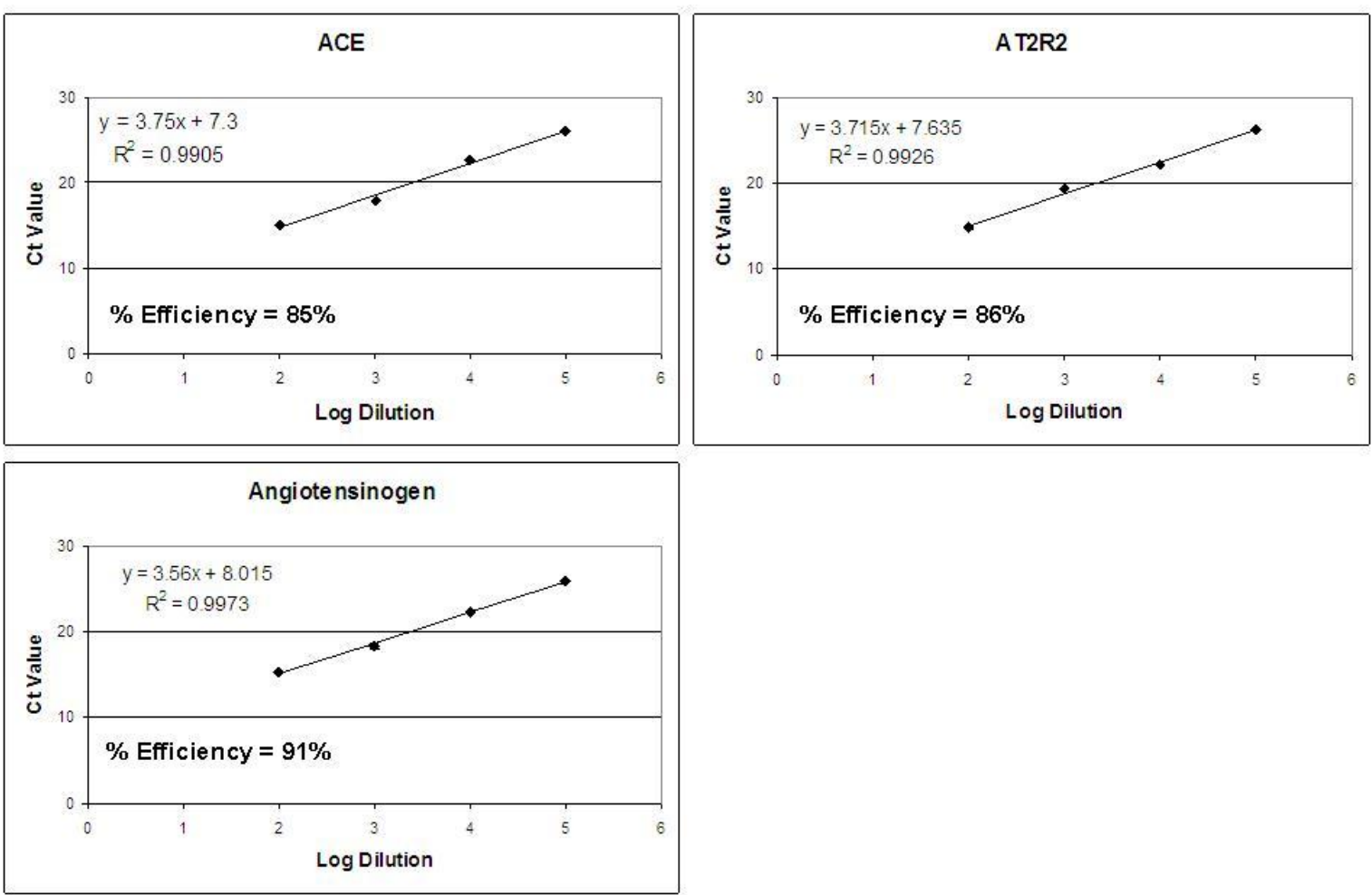

Figure 28: Efficiencies of the primers used to assess markers of the RAS. Purified PCR products were diluted and amplified in duplicate, and the mean Ct value plotted against the log of the dilution. The slope of the best fit line was used to calculate primer efficiencies (displayed on the graph). $R^{2}$ values demonstrate the fit of the trend line to the data. 

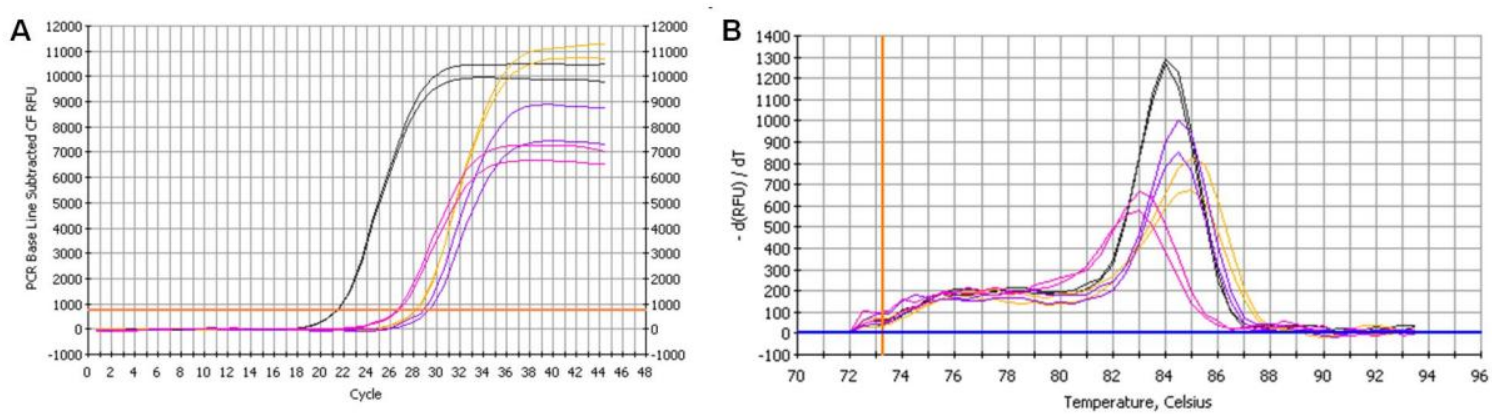

C
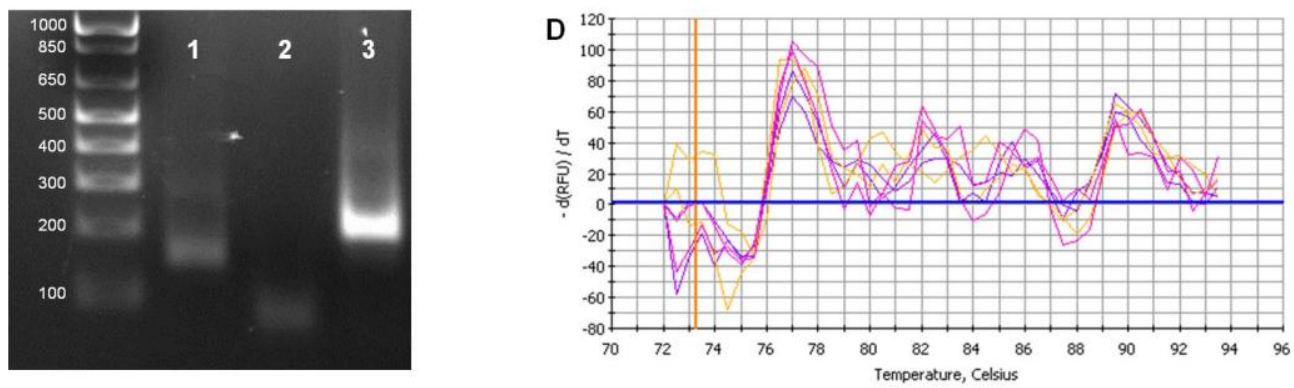

Figure 29: Representative real-time PCR data used to assess the RAS. (A) Representative trace image of data acquired by real-time PCR for target genes used in this chapter. All genes (cyclophilin A, black; angiotensinogen, pink; AT2R2, gold; ACE, purple) were analysed in duplicate to ensure the accuracy of the results. (B) Melt-curve profile analysis of these samples revealed single sharp peaks for cyclophilin $A\left(84^{\circ} \mathrm{C}\right)$, angiotensinogen $\left(83^{\circ} \mathrm{C}\right), \mathrm{AT} 2 \mathrm{R} 2\left(85^{\circ} \mathrm{C}\right)$ and $\mathrm{ACE}$ $\left(84.5^{\circ} \mathrm{C}\right)$. Low levels of product were also detected at $73-82^{\circ} \mathrm{C}$ for all primer pairs. (C) Single PCR products were validated according to size using agarose gel electrophoresis (lane 1, ACE; lane 2, angiotensinogen; lane 3, AT2R2). (D) Melt-curve profile analysis of negative controls (PCR reactions completed with template replaced with either ddH2O or equi-molar non-reverse transcribe RNA) showed no specific PCR product for any of the genes analysed.

Mean $\Delta \mathrm{Ct}$ values for each transcript were used to compare relative transcript abundance between treatment and training groups (Table 9). From these data, $\Delta \Delta \mathrm{Ct}$ values were calculated relative to vehicle-treated, untrained animals for presentation purposes (Fig. 30). No statistically significant effects of drug treatment were observed in either trained or untrained animals, nor did training affect gene expression in either THC-treated or vehicle-treated animals. However, a trend towards significance was observed in the latter $(p=0.096)$ in which training of vehicle-treated but not THC-treated animals appeared to increase ACE expression. This may indicate that training increases the concentration of Angll in the hippocampus and this effect is blocked by THC. Angll and its derivatives have been implicated in the modulation of a variety of mnemonic functions, including 
LTP, memory consolidation and the beneficial effect of voluntary exercise on learning and memory (Hellner, Walther et al. 2005; Kerr, Bevilaqua et al. 2005; Braszko, Walesiuk et al. 2006; Akhavan, Emami-Abarghoie et al. 2008). It is difficult, however, to draw any meaningful conclusions from these data as no clear trends are observed and the errors are significantly larger than those presented in Chapters 4 and 5. Perhaps further optimisation of the PCR reaction for these transcripts would provide a more accurate resolution of the data. For example, in some instances the combined errors associated with statistical measurement were greater than an $80 \%$ difference between means, approximately double that observed in the data presented in previous chapters.

\begin{tabular}{|c|c|c|c|c|}
\hline \multirow{2}{*}{ Target } & \multicolumn{2}{|c|}{ Trained Animals } & \multicolumn{2}{|c|}{ Untrained Animals } \\
\hline & Vehicle-Treated & THC-Treated & Vehicle-Treated & THC-Treated \\
\hline \multirow{2}{*}{ ACE } & $7.28 \pm 0.42$ & $8.54 \pm 0.53$ & $8.16 \pm 0.43$ & $8.30 \pm 0.35$ \\
\hline & $(1.84 X)$ & $(0.85 X)$ & & $(0.91 X)$ \\
\hline \multirow{2}{*}{ Angiotensinogen } & $5.96 \pm 0.29$ & $5.88 \pm 0.20$ & $5.39 \pm 0.37$ & $5.71 \pm 0.39$ \\
\hline & $(0.67 X)$ & $(0.89 X)$ & & $(0.80 X)$ \\
\hline \multirow{2}{*}{ AT2R2 } & $6.86 \pm 0.47$ & $7.41 \pm 0.49$ & $7.42 \pm 0.17$ & $6.76 \pm 0.37$ \\
\hline & $(1.47 X)$ & $(0.64 X)$ & & $(1.58 X)$ \\
\hline
\end{tabular}

\footnotetext{
Table 9: Relative gene expression levels between treatment and behavioural groups for

markers of the RAS. Data are mean \pm SEM $\Delta$ Ct values. Fold change relative to untrained animals of the same treatment (for trained animals), or vehicle-treated animals (for untrained THC-treated animals) are shown in parenthesis.
}

In an effort to overcome the apparent limitations of the PCR reactions for these transcripts, attempts were made to quantify the protein for ACE and AT2R2 using Western blotting. However, the antibodies used were found to be of poor quality, in that they either detected their targets inconsistently (for ACE) or failed to detect them altogether (AT2R2). The investigation into whether cannabis may perturb cognition by interfering with the RAS was therefore abandoned because of the 
financial and time restraints. It would be interesting, however, to investigate this possibility using an improved PCR reaction and better quality antibodies.

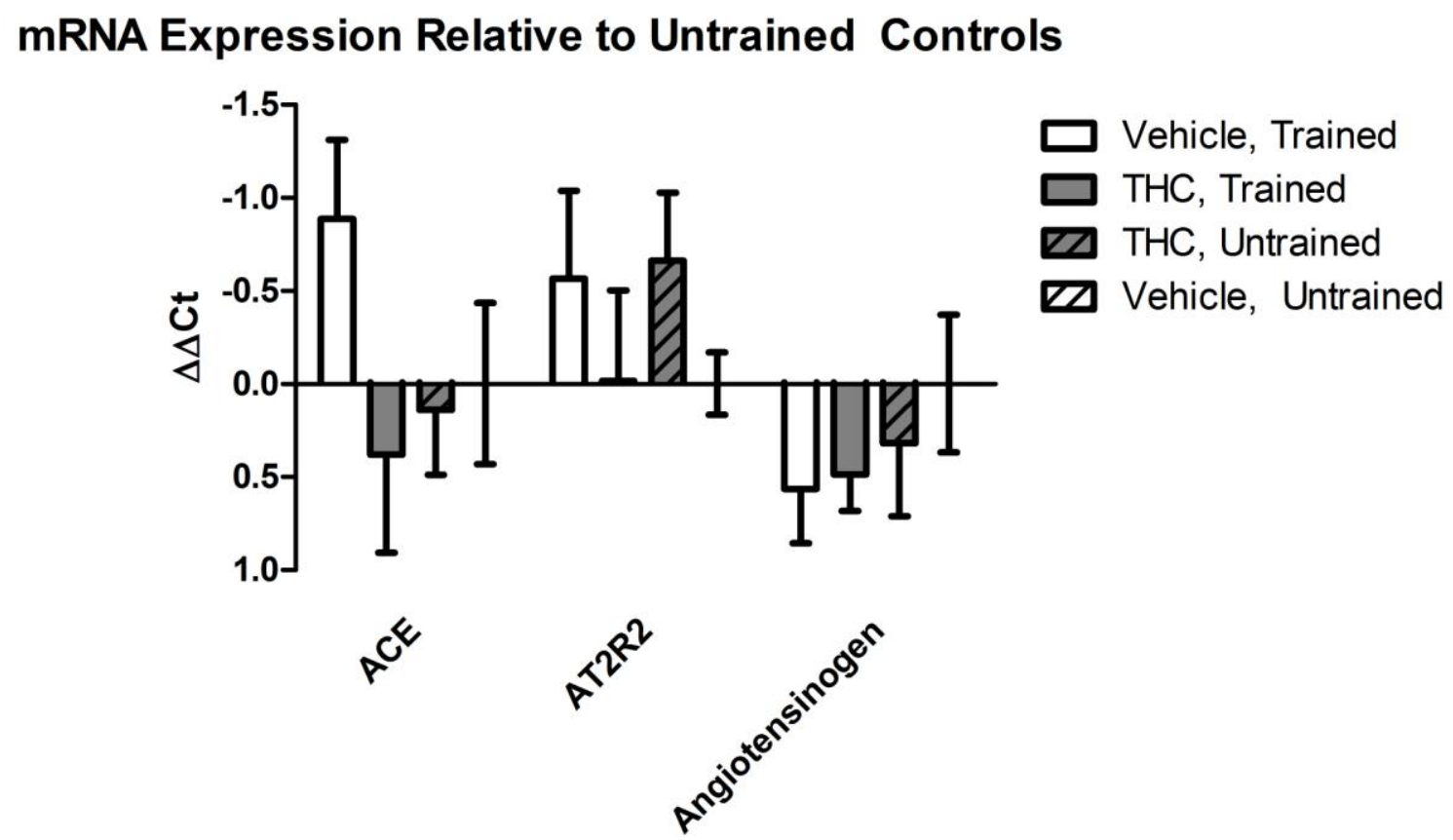

Figure 30: Neither training nor drug treatment had any significant effect on the expression of genes of the RAS. Data are mean \pm SEM $\Delta \Delta$ Ct values based on $n=8$ trained and $n=5$ untrained vehicle-treated animals, and $n=7$ trained and $n=5$ untrained THC-treated animals. 


\section{Appendix $6 \quad$ Whole Gel Images of Western-blots}

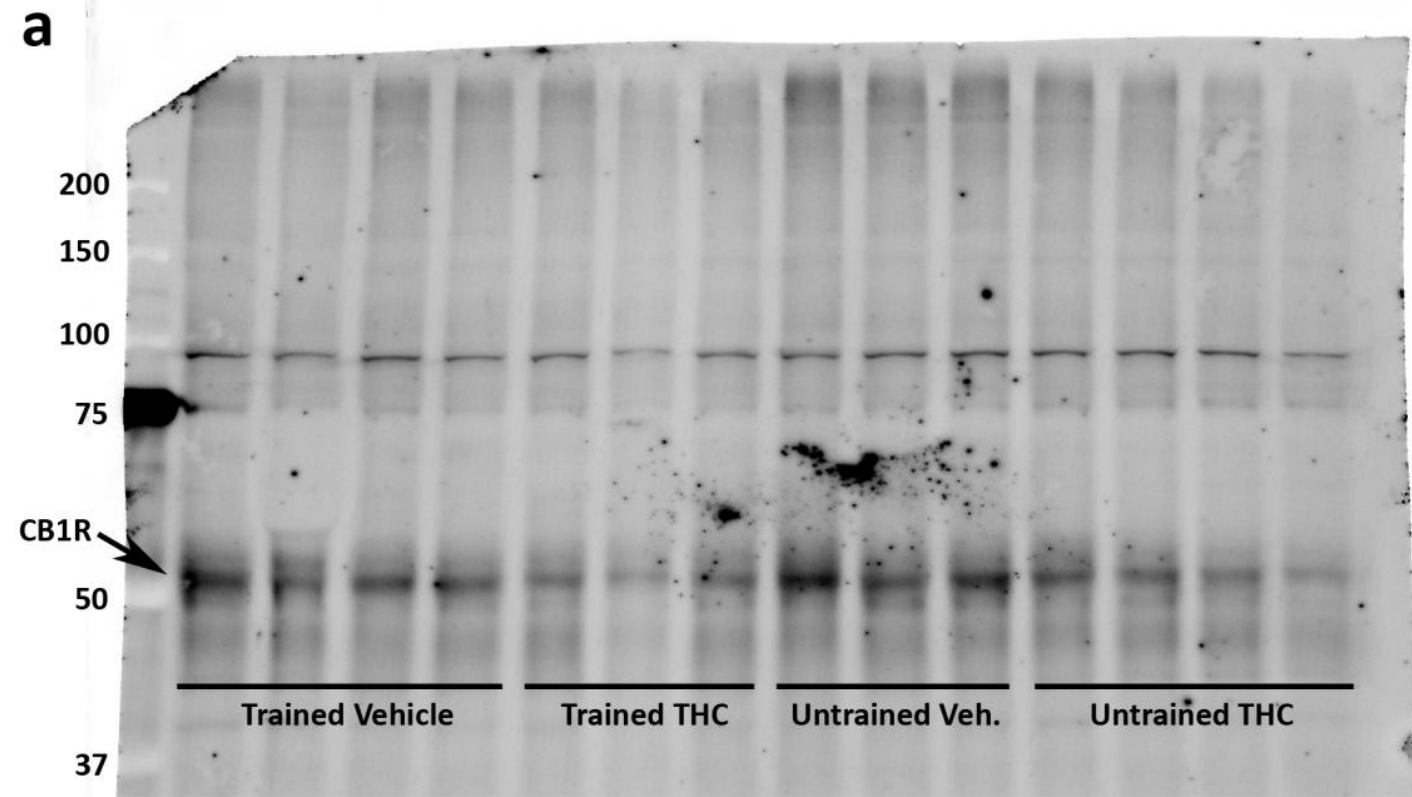

GAPDH

b

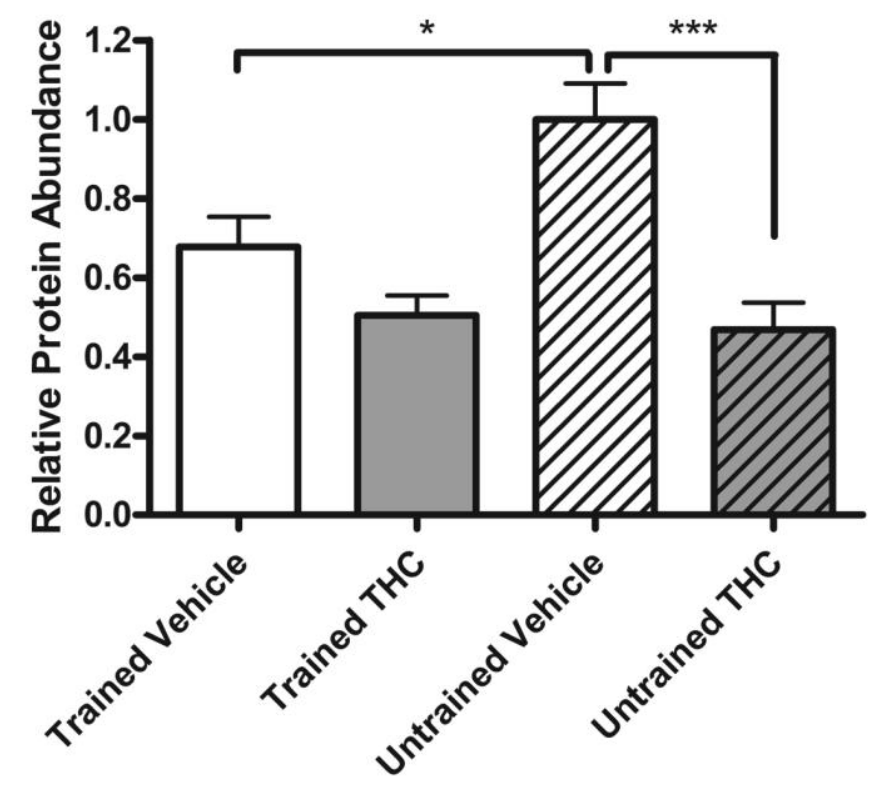

Figure 31: Western-blot of CB1R. A) Representative gel used to determine the protein abundance of CB1R. B) Western-blot data normalised to GAPDH. ${ }^{*} p<0.05,{ }^{* *} p<0.001$ by one-way ANOVA with Bonferroni post-hoc testing. 


\section{a}

PSA-

NCAM

250

150

100

Trained Vehicle

Trained THC

Untrained Veh.

Untrained THC

50

37

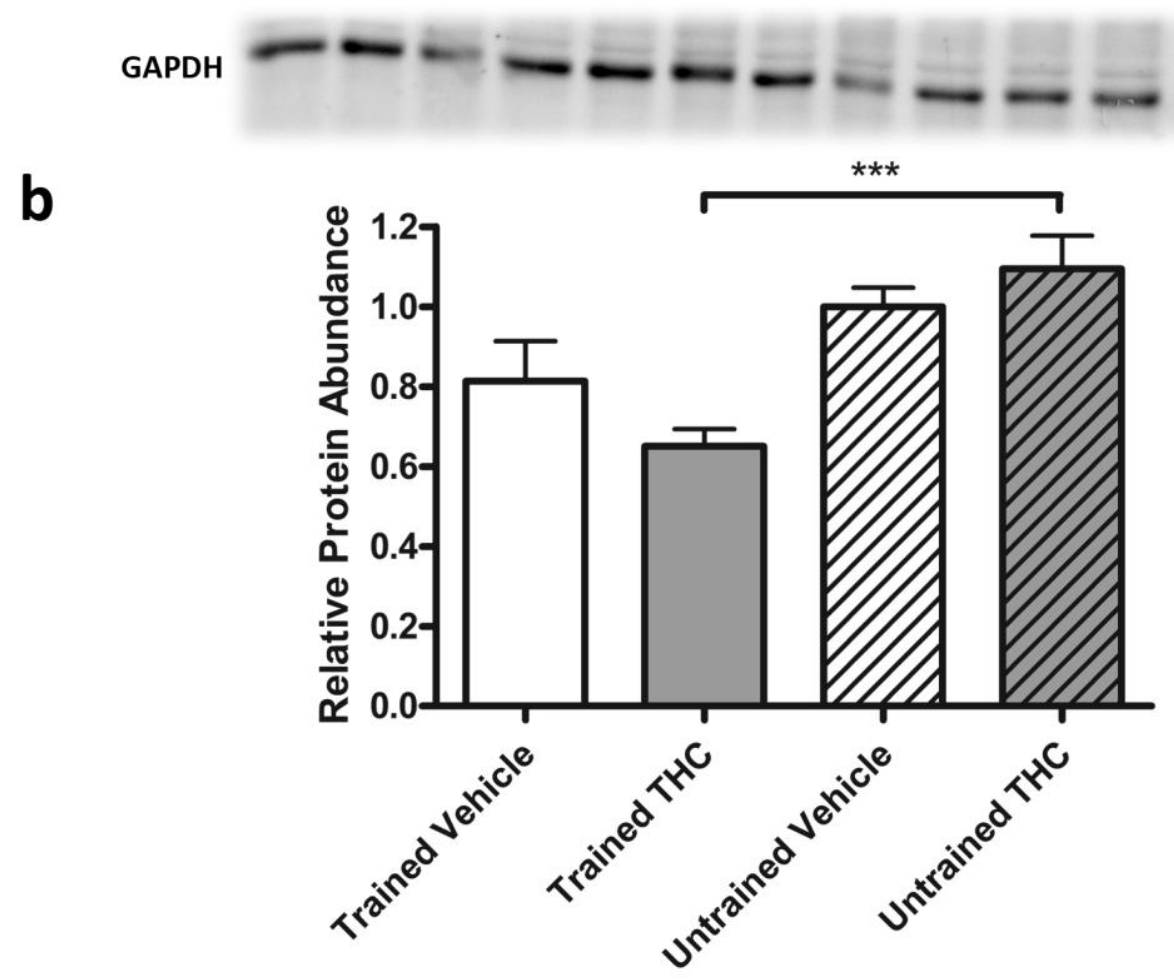

Figure 32: Western-blot of PSA-NCAM. A) Representative gel used to determine the protein abundance of PSA-NCAM. B) Western-blot data normalised to GAPDH. ${ }^{* *} p<0.001$ by one-way ANOVA with Bonferroni post-hoc testing. 
a
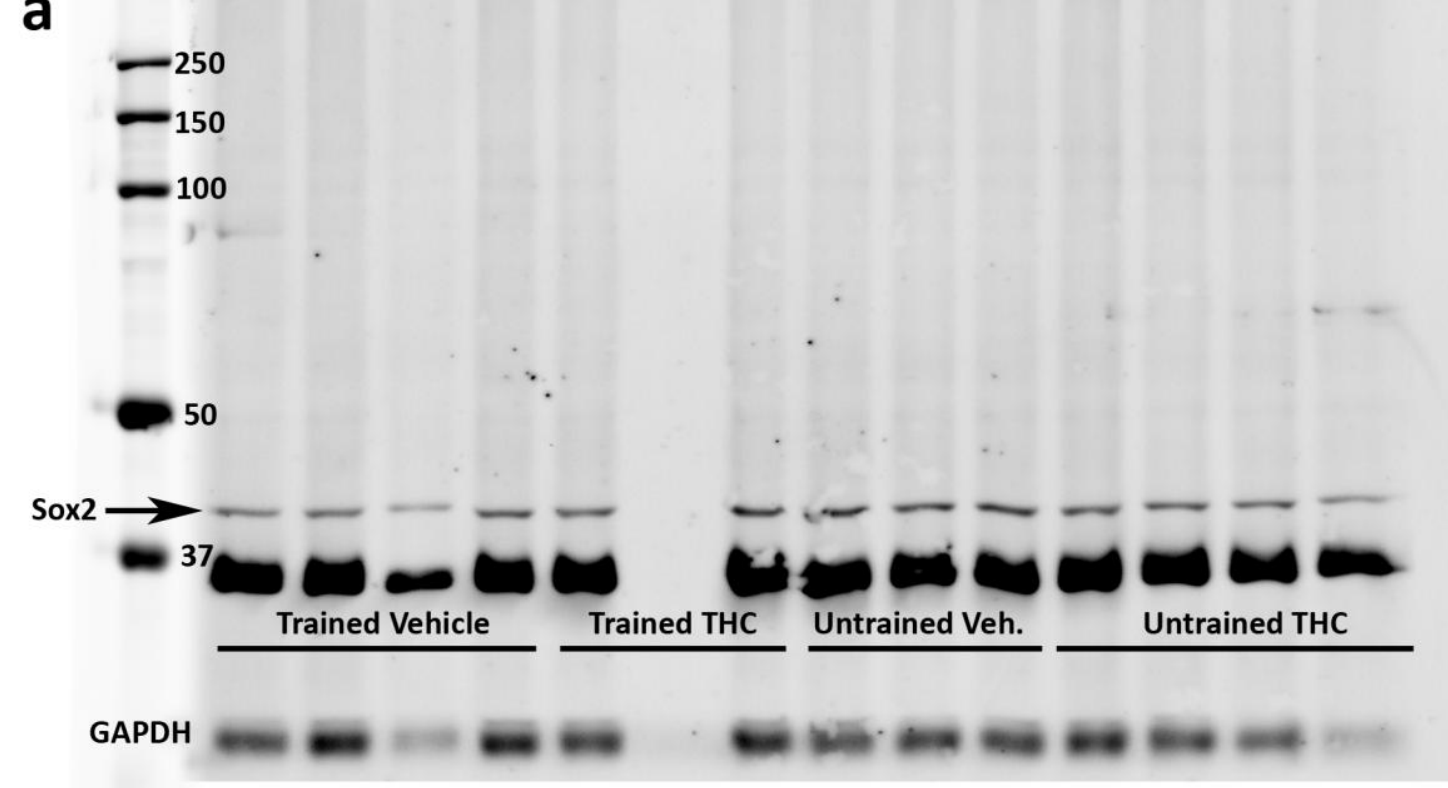

b

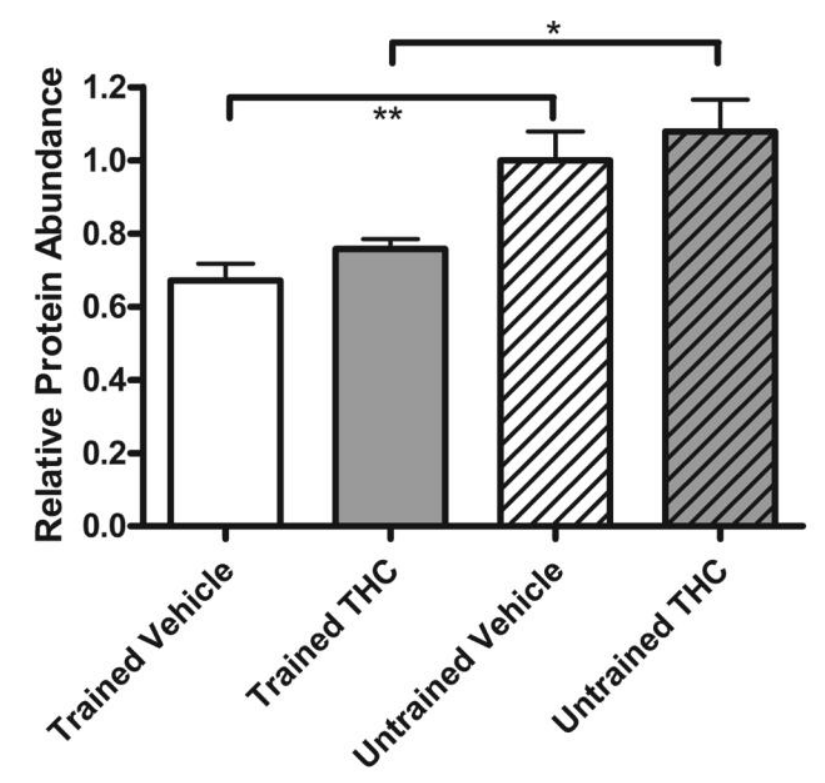

Figure 33: Western-blot of Sox2. A) Representative gel used to determine the protein abundance of Sox2. B) Western-blot data normalised to GAPDH. ${ }^{*} p<0.05,{ }^{* *} p<0.01$ by one-way ANOVA with Bonferroni post-hoc testing. 
a

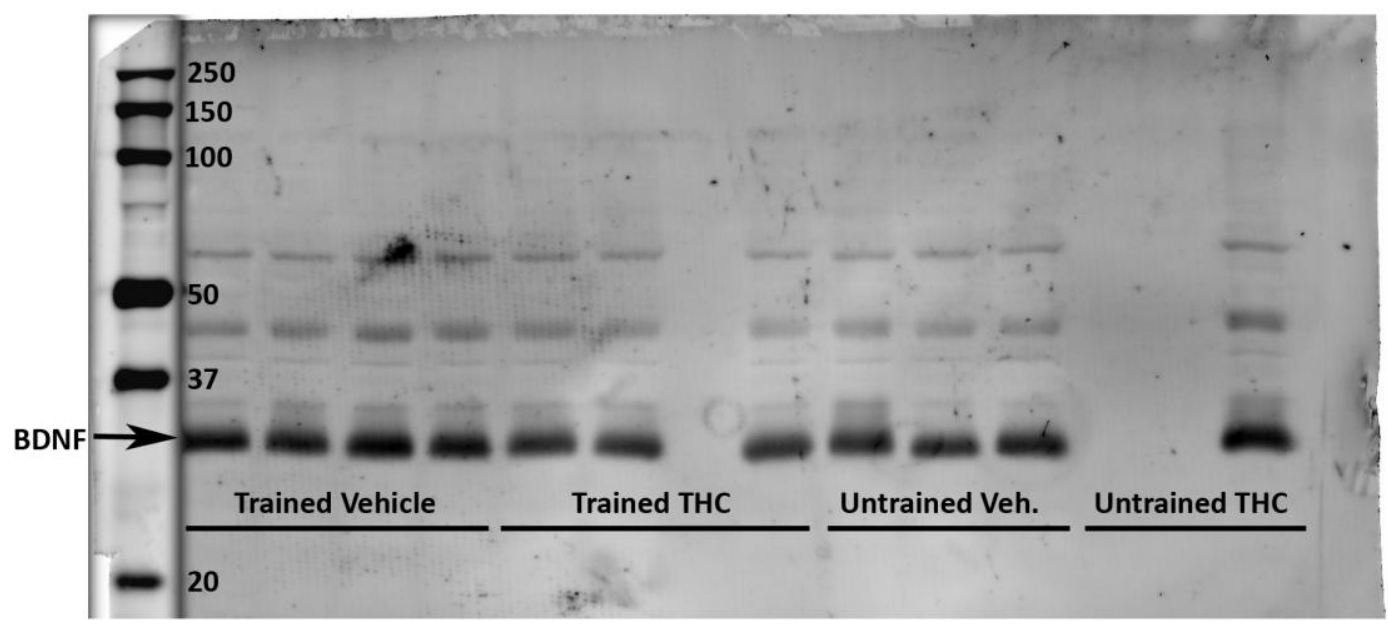

GAPDH

b

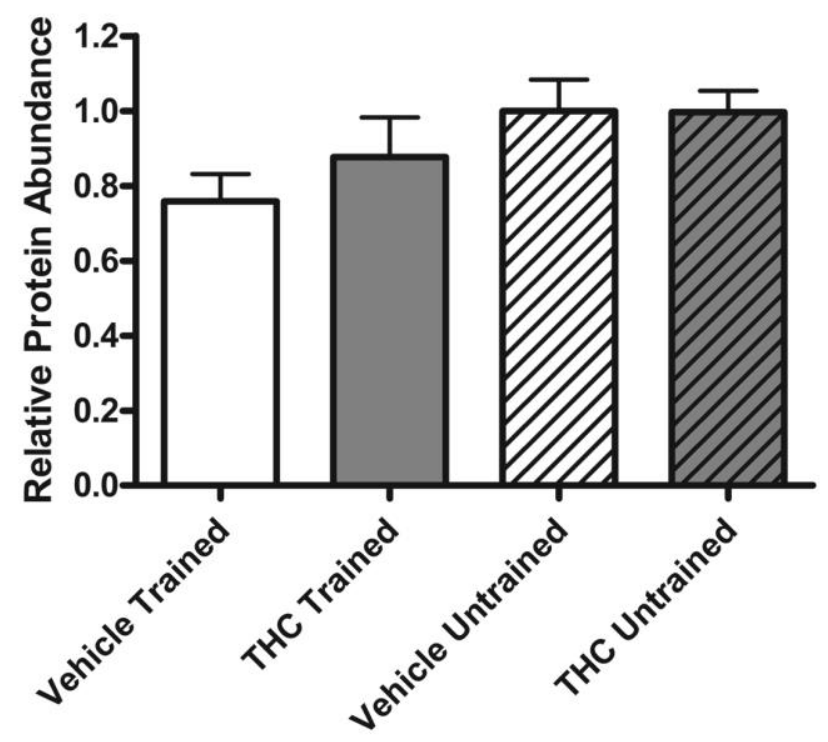

Figure 34: Western-blot of BDNF. A) Representative gel used to determine the protein abundance of BDNF. B) Western-blot data normalised to GAPDH. 


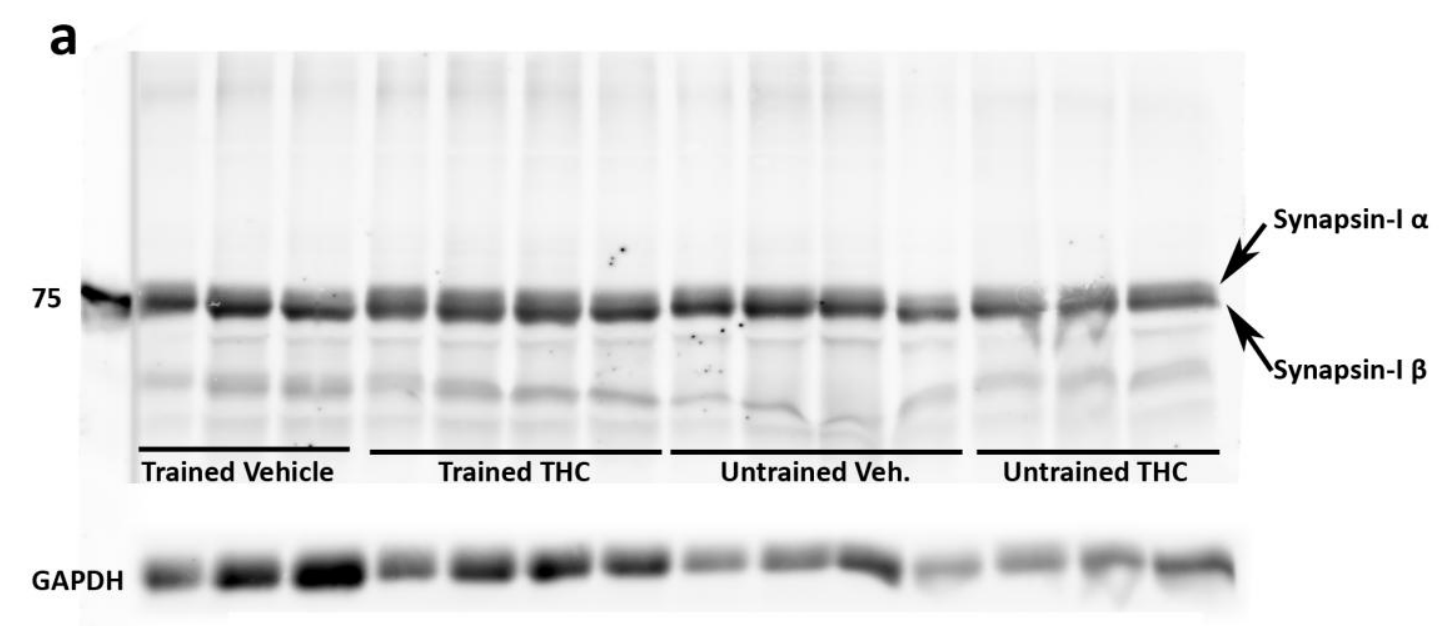

b

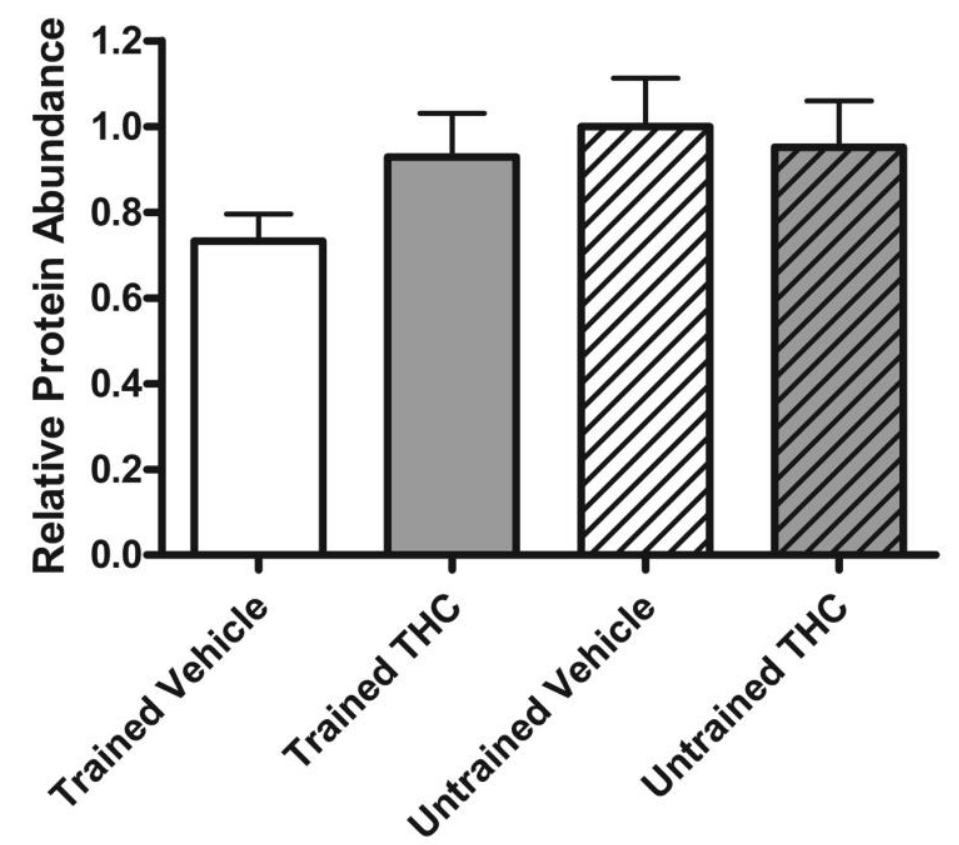

Figure 35: Western-blot of Synapsin-I. A) Representative gel used to determine the protein abundance of Synapsin-I. B) Western-blot data normalised to GAPDH. 

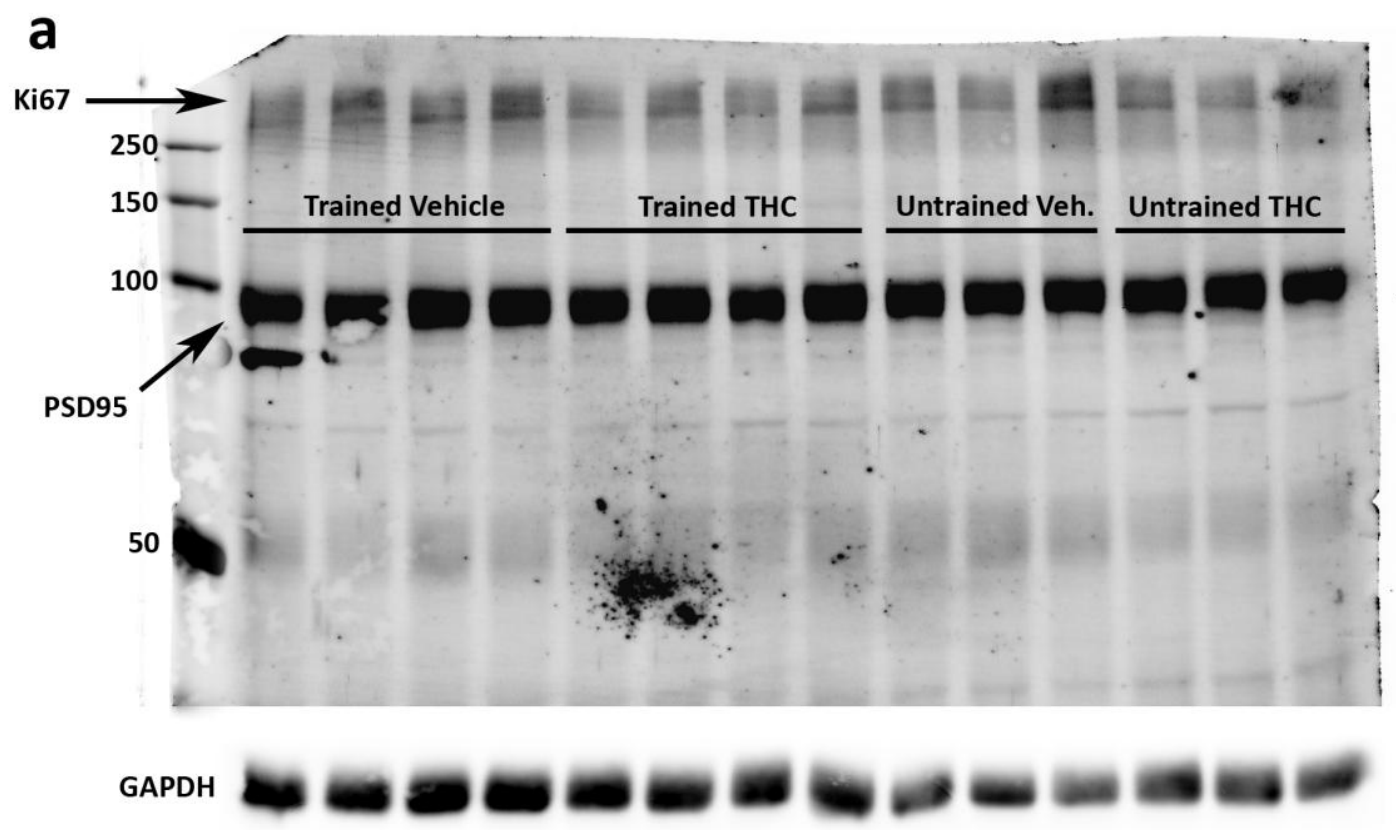

b

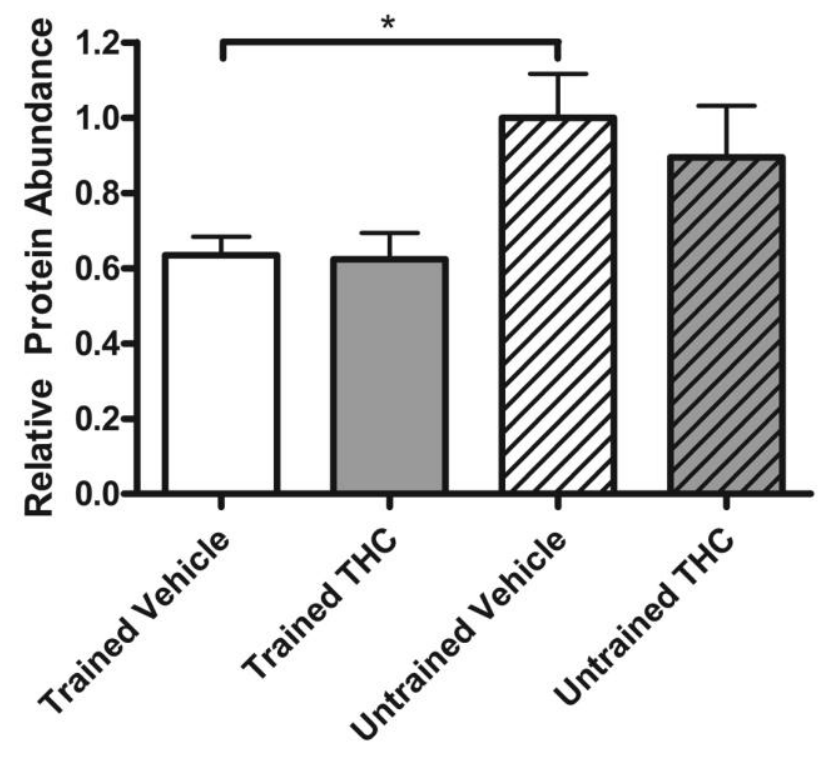

Figure 36: Western-blot of Ki67. A) Representative gel used to determine the protein abundance of Ki67 in this study. B) Western-blot data normalised to GAPDH. ${ }^{*} p<0.05$ by one-way ANOVA with Bonferroni post-hoc testing. 


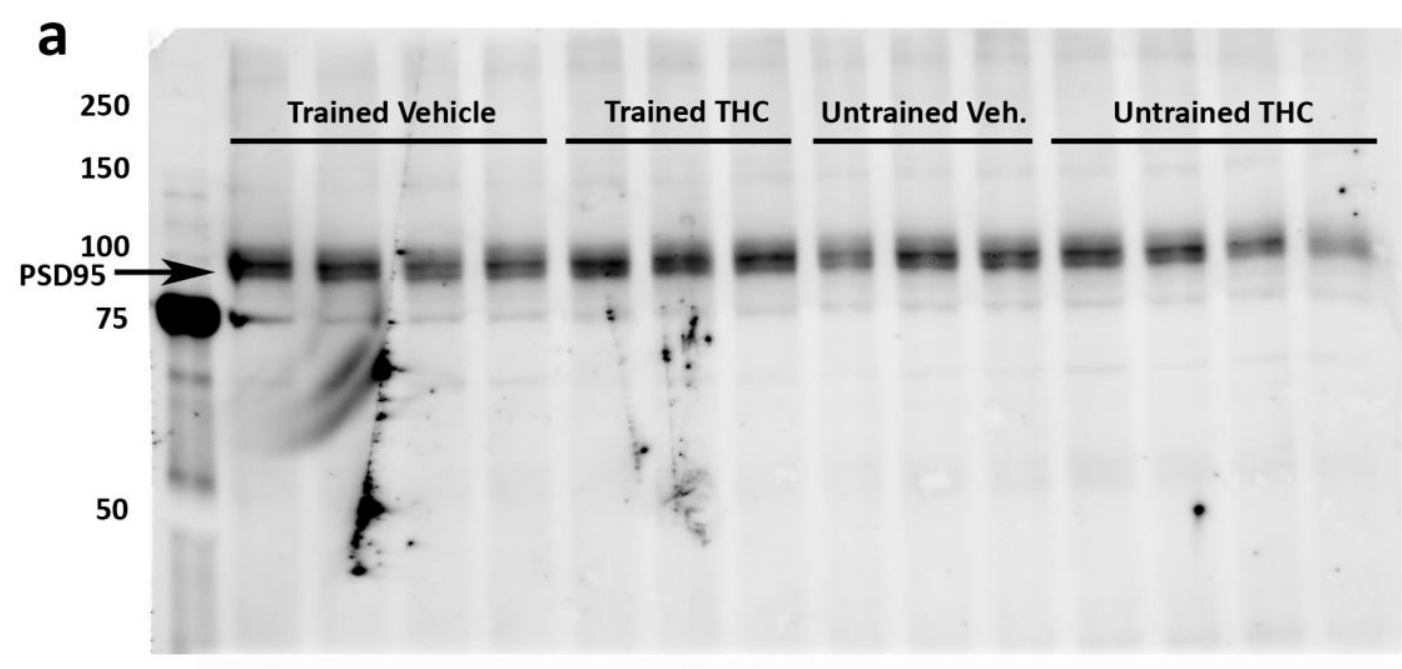

GAPDH

b

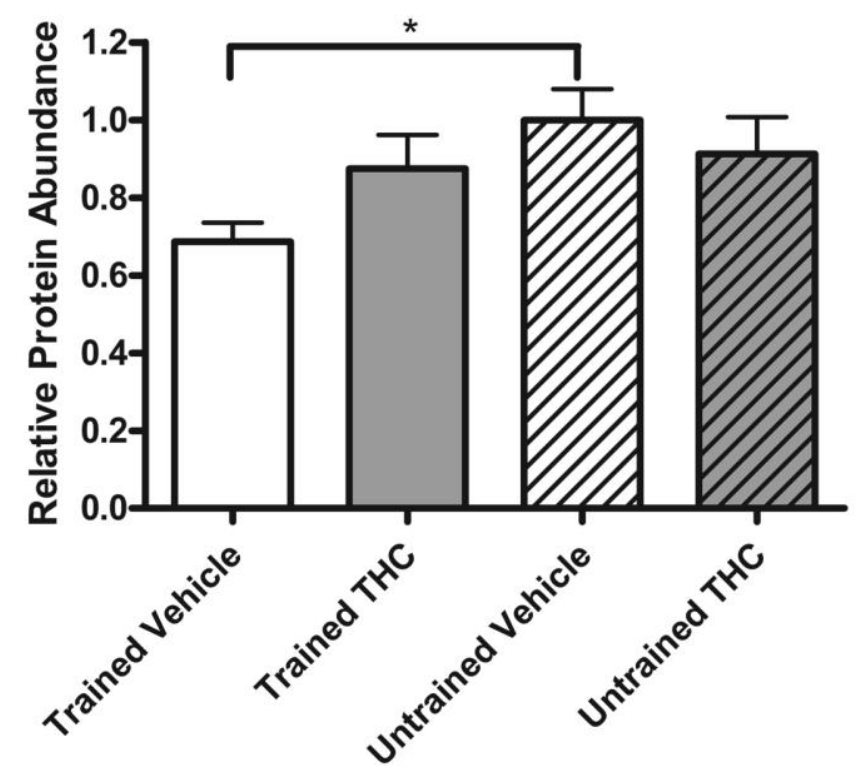

Figure 37: Western-blot of PSD95. A) Representative gel used to determine the protein abundance of PSD95 in this study. B) Western-blot data normalised to GAPDH. ${ }^{*} p<0.05$ by oneway ANOVA with Bonferroni post-hoc testing. 


\section{Appendix $7 \quad$ Validation of CB1R Antibody}

Antibodies raised against specific target proteins are a useful and powerful tool in the localisation and quantification of proteins of interest. The quality of these analyses is however, ultimately limited by the quality of the antibodies used. In the case of antibodies raised against CB1R, a number of the commercially available antibodies have been reported to give unsatisfactory results. Specifically, the staining patterns observed in immunohistochemistry did not match with the known distribution of CB1R in a variety of brain sections, and the antibodies detected non-specific proteins of a similar size to CB1R (Grimsey, Goodfellow et al. 2008). The anti-CB1R antibody used in this study (Alomone Labs) was not assessed in the study of Grimsey et al (2008), and as such their findings do not apply to this antibody. However, the specificity of the Alomone Labs anti-CB1R antibody used in this analysis was assessed by immunohistochemistry and Western-blot using the criteria of Grimsey et al (2008). Strong staining was observed surrounding the dentate gyrus, consistent with previous reports of CB1R localisation in the hippocampus (Glass, Dragunow et al. 1997), and a single species was detected within the monomeric CB1R range of $\sim 50-60 \mathrm{kDa}$ on Western-blots (Fig. 38). These results compare favourably to the antibodies tested by Grimsey et al (2008), and suggest that the Alomone Labs anti-CB1R antibody is suitable for the specific detection of CB1R in this study. 
A

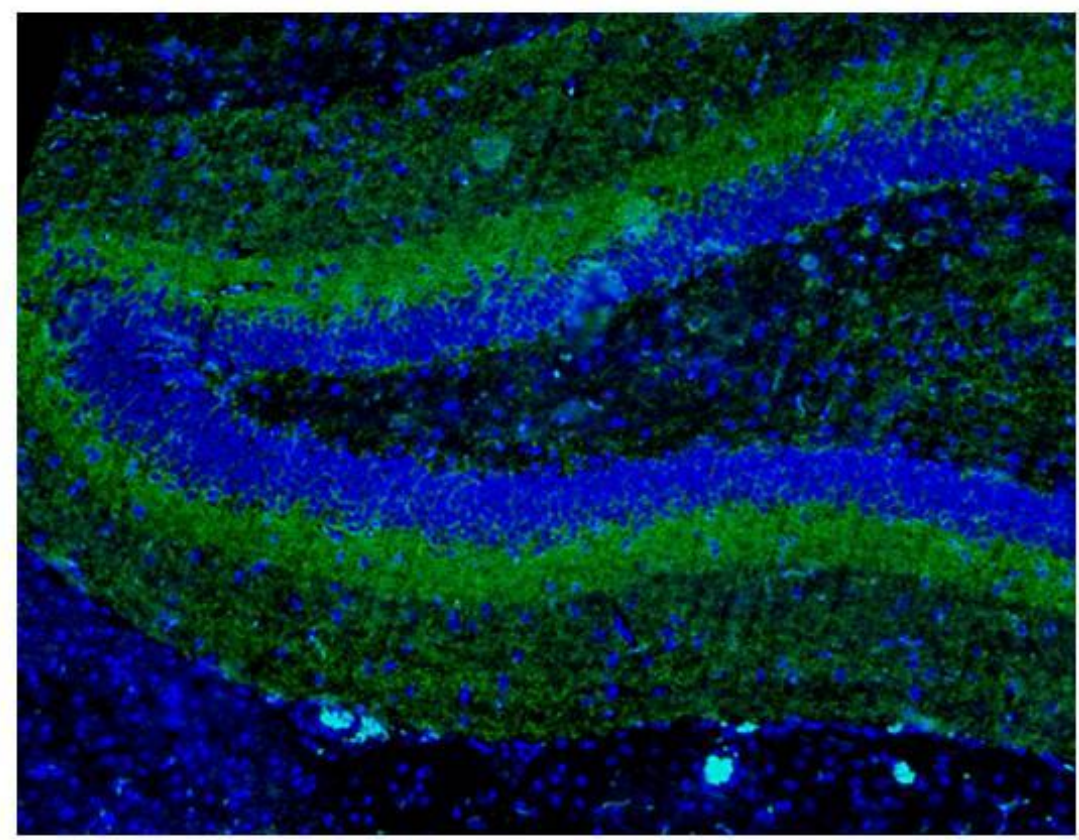

B

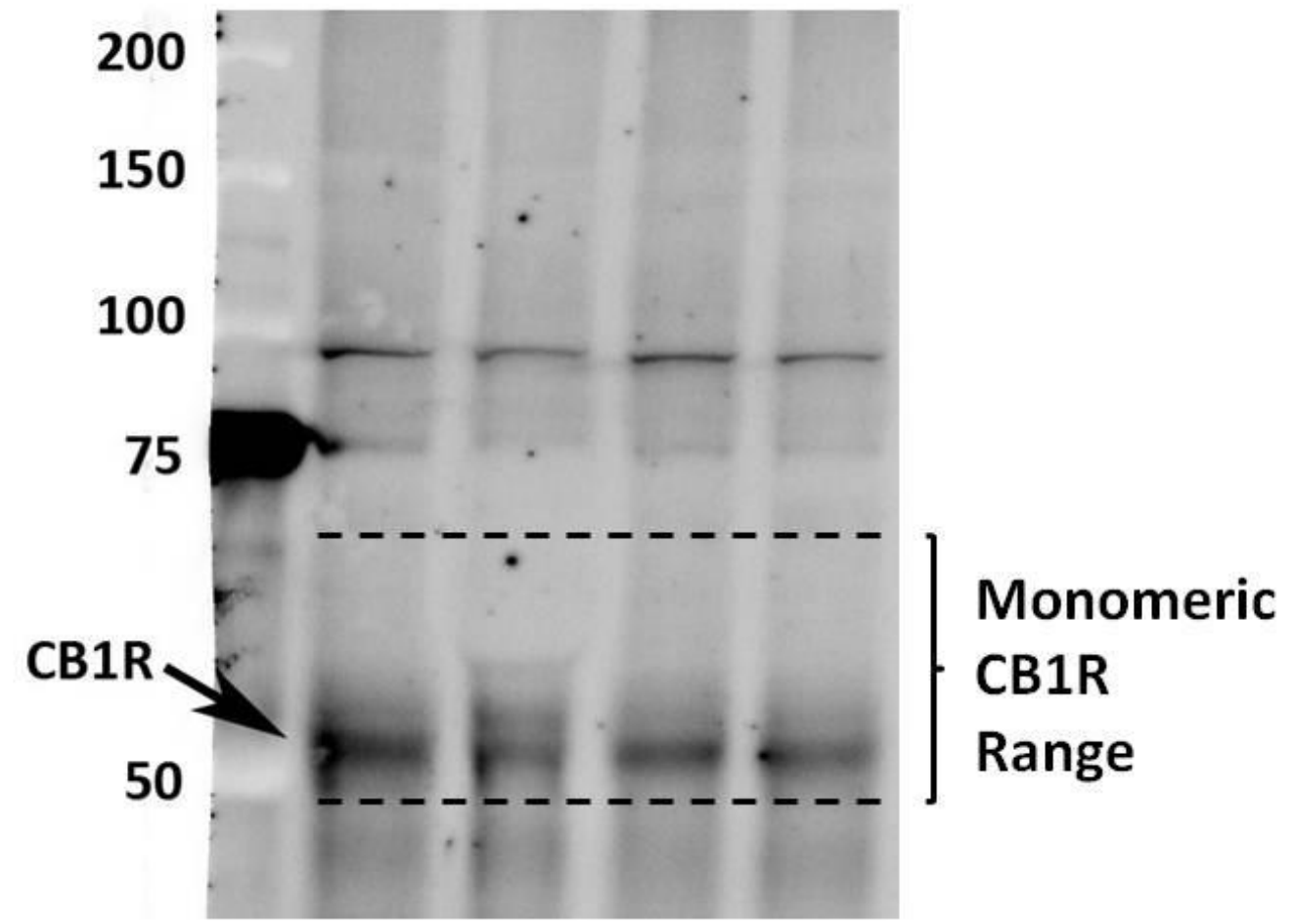

Figure 38: Validation of CB1R antibody. A) CB1R (green) is strongly expressed surrounding the dentate gyrus of the hippocampus (cell nuclei, blue), consistent with the accepted distribution of $\mathrm{CB} 1 \mathrm{R}$ in the hippocampus. B) Only a single protein within the monomeric range of CB1R ( 50 - 60 $\mathrm{kDa}$ ) was detected using the Alomone Labs anti-CB1R antibody. 


\section{Chapter 8 Reference List}

(2003). Drug Use in New Zealand: Analysis of the 2003 New Zealand Health Behavious Survey Drug Use, Ministry of Health.

Aceto, M. D., S. M. Scates, J. A. Lowe and B. R. Martin (1996). "Dependence on Delta(9)tetrahydrocannabinol: Studies on precipitated and abrupt withdrawal." Journal of Pharmacology and Experimental Therapeutics 278(3): 1290-1295.

Adriani, W. and G. Laviola (2004). "Windows of vulnerability to psychopathology and therapeutic strategy in the adolescent rodent model." Behavioural Pharmacology 15(5-6): 341-352.

Aguado, T., K. Monory, J. Palazuelos, N. Stella, B. Cravatt, B. Lutz, et al. (2005). "The endocannabinoid system drives neural progenitor proliferation." Faseb Journal 19(9): 1704-+.

Aguado, T., J. Palazuelos, K. Monory, N. Stella, B. Cravatt, B. Lutz, et al. (2006). "The endocannabinoid system promotes astroglial differentiation by acting on neural progenitor cells." Journal of Neuroscience 26(5): 1551-1561.

Akhavan, M. M., M. Emami-Abarghoie, B. Sadighi-Moghaddam, M. Safari, Y. Yousefi and A. Rashidy-Pour (2008). "Hippocampal angiotensin II receptors play an important role in mediating the effect of voluntary exercise on learning and memory in rat." Brain Research 1232: 132-138.

Alger, B. E. and T. A. Pitler (1995). "Retrograde Signaling at GABA(A)-Receptor Synapses in the Mammalian CNS." Trends in Neurosciences 18(8): 333-340.

Altman, J. and G. D. Das (1965). "Post-Natal Origin of Microneurones in Rat Brain." Nature 207(5000): 953-\&.

Ambrogini, P., R. Cuppini, C. Cuppini, S. Ciaroni, T. Cecchini, P. Ferri, et al. (2000). "Spatial learning affects immature granule cell survival in adult rat dentate gyrus." Neuroscience Letters 286(1): 21-24.

Ameri, A. (1999). "The effects of cannabinoids on the brain." Progress in Neurobiology 58(4): 315348.

Ameri, A., A. Wilhelm and T. Simmet (1999). "Effects of the endogeneous cannabinoid, anandamide, on neuronal activity in rat hippocampal slices." British Journal of Pharmacology 126(8): 1831-1839.

Andersen, P. (1990). "Synaptic Integration in Hippocampal CA1 Pyramids." Progress in Brain Research 83: 215-222.

Anderson, G. D. (2010). "Developmental Pharmacokinetics." Seminars in Pediatric Neurology 17(4): 208-213.

Applied-Biosystems (2006). "Application Note: Amplification Effieiency of TaqMan Gene Expression Assays."

Arata, S., T. W. Klein, C. Newton and H. Friedman (1991). "Tetrahydrocannabinol Treatment Suppresses Growth Restriction of Lefionella-Pneumophila in Murine Macrophage Cultures." Life Sciences 49(6): 473-479.

Auclair, N., S. Otani, P. Soubrie and F. Crepel (2000). "Cannabinoids modulate synaptic strength and plasticity at glutamatergic synapses of rat prefrontal cortex pyramidal neurons." Journal of Neurophysiology 83(6): 3287-3293.

Bach, M. E., M. Barad, H. Son, M. Zhuo, Y. F. Lu, R. Shih, et al. (1999). "Age-related defects in spatial memory are correlated with defects in the late phase of hippocampal long-term potentiation in vitro and are attenuated by drugs that enhance the cAMP signaling pathway." Proceedings of the National Academy of Sciences of the United States of America 96(9): 5280-5285.

Bailey, C. H. and E. R. Kandel (1993). "Structural-Changes Accompanying Memory Storage." Annual Review of Physiology 55: 397-426. 
Bammer, G. (1982). "Pharmacological Investigations of Neurotransmitter Involvement in PassiveAvoidance Responding - a Review and some New Results." Neuroscience and Biobehavioral Reviews 6(3): 247-296.

Barna, I., K. Soproni, A. Arszovszki, K. Csabai and J. Haller (2007). "WIN-55,212-2 chronically implanted into the CA3 region of the dorsal hippocampus impairs learning: a novel method for studying chronic, brain-area-specific effects of cannabinoids." Behavioural Pharmacology 18(5-6): 515-520.

Beltramo, M. and D. Piomelli (2000). "Carrier-mediated transport and enzymatic hydrolysis of the endogenous cannabinoid 2-arachidonylglycerol." Neuroreport 11(6): 1231-1235.

Benke, T. A., A. Luthi, J. T. R. Isaac and G. L. Collingridge (1998). "Modulation of AMPA receptor unitary conductance by synaptic activity." Nature 393(6687): 793-797.

Berghuis, P., A. M. Rajnicek, Y. M. Morozov, R. A. Ross, J. Mulder, G. M. Urban, et al. (2007). "Hardwiring the brain: Endocannabinoids shape neuronal connectivity." Science 316(5828): 1212-1216.

Bhardwaj, R. D., M. A. Curtis, K. L. Spalding, B. A. Buchholz, D. Fink, T. Bjork-Eriksson, et al. (2006). "Neocortical neurogenesis in humans is restricted to development." Proceedings of the National Academy of Sciences of the United States of America 103(33): 12564-12568.

Bisogno, T., M. Maccarrone, L. De Petrocellis, A. Jarrahian, A. Finazzi-Agro, C. Hillard and V. Di Marzo (2001). "The uptake by cells of 2-arachidonoylglycerol, an endogenous agonist of cannabinoid receptors." European Journal of Biochemistry 268(7): 1982-1989.

Blanchard, D. K., C. Newton, T. W. Klein, W. E. Stewart and H. Friedman (1986). "In Vitro and In Vivo Suppressive Effects of Delta-9-Tetrahydrocannabinol on Interferon-Production by Murine Spleen-Cells." International Journal of Immunopharmacology 8(7): 819-824.

Block, F., M. Kunkel and M. Schwarz (1993). "Quinolinic Acid Lesion of the Striatum Induces Impairment in Spatial-Learning and Motor-Performance in Rats." Neuroscience Letters 149(2): 126-128.

Bonner, T. I., N. J. Buckley, A. C. Young and M. R. Brann (1987). "Identification of a Family of Muscarinic Acetylcholine-Receptor Genes." Science 237(4814): 527-532.

Braszko, J. J., A. Walesiuk and P. Wielgat (2006). "Cognitive effects attributed to angiotensin II may result from its conversion to angiotensin IV." Journal of the Renin-AngiotensinAldosterone System 7(3): 168-174.

Breivogel, C. S., S. R. Childers, S. A. Deadwyler, R. E. Hampson, L. J. Vogt and L. J. Sim-Selley (1999). "Chronic Delta(9)-tetrahydrocannabinol treatment produces a time-dependent loss of cannabinoid receptors and cannabinoid receptor-activated $\mathrm{G}$ proteins in rat brain." Journal of Neurochemistry 73(6): 2447-2459.

Brisson, M., T. Larissa, R. Park and K. Hamby (2000). "Identification of nonspecific products using melt-curve analysis." Bio-Rad Laboratories, Inc. Technical note 2684.

Brown, M. F. and G. W. Giumetti (2006). "Spatial pattern learning in the radial arm maze." Learning \& Behavior 34(1): 102-108.

Brown, M. F. and M. Terrinoni (1996). "Control of choice by the spatial configuration of goals." Journal of Experimental Psychology-Animal Behavior Processes 22(4): 438-446.

Calverley, R. K. S. and D. G. Jones (1990). "Contributions of Dendritic Spines and Perforated Synapses to Synaptic Plasticity." Brain Research Reviews 15(3): 215-249.

Castellano, C., S. Cabib, A. Palmisano, V. Di Marzo and S. Puglisi-Allegra (1997). "The effects of anandamide on memory consolidation in mice involve both D-1 and D-2 dopamine receptors." Behavioural Pharmacology 8(8): 707-712.

Castillo, P. E., R. Janz, T. C. Sudhof, T. Tzounopoulos, R. C. Malenka and R. A. Nicoll (1997). "Rab3A is essential for mossy fibre long-term potentiation in the hippocampus." Nature 388(6642): 590-593. 
Ceccaldi, P. E., F. Grohovaz, F. Benfenati, E. Chieregatti, P. Greengard and F. Valtorta (1995). "Dephosphorylated Synapsin-I Anchors Synaptic Vesicles To Actin Cytoskeleton - An Analysis By Videomicroscopy." Journal of Cell Biology 128(5): 905-912.

Cha, Y. M., K. H. Jones, C. M. Kuhn, W. A. Wilson and H. S. Swartzwelder (2007). "Sex differences in the effects of Delta(9)-tetrahydrocannabinol on spatial learning in adolescent and adult rats." Behavioural Pharmacology 18(5-6): 563-569.

Cha, Y. M., A. M. White, C. M. Kuhn, W. A. Wilson and H. S. Swartzwelder (2006). "Differential effects of delta(9)-THC on learning in adolescent and adult rats." Pharmacology Biochemistry and Behavior 83(3): 448-455.

Chavez, A. E., C. Q. Chiu and P. E. Castillo (2010). "TRPV1 activation by endogenous anandamide triggers postsynaptic long-term depression in dentate gyrus." Nature Neuroscience 13(12): 1511-U99.

Chevaleyre, V. and P. E. Castillo (2004). "Endocannabinoid-mediated metaplasticity in the hippocampus." Neuron 43(6): 871-881.

Childers, S. R., L. Fleming, C. Konkoy, D. Marckel, M. Pacheco, T. Sexton and S. Ward (1992). "Opioid and Cannabinoid Receptor Inhibition of Adenylyl Cyclase in Brain." Annals of the New York Academy of Sciences 654: 33-51.

Chin, L. S., L. Li, A. Ferreira, K. S. Kosik and P. Greengard (1995). "Impairment of axonal development and of synaptogenesis in hippocampal neurons of synapsin I-deficient mice." Proceedings of the National Academy of Sciences of the United States of America 92(20): 9230-9234.

Cho, Y. H., E. Friedman and A. J. Silva (1999). "Ibotenate lesions of the hippocampus impair spatial learning but not contextual fear conditioning in mice." Behavioural Brain Research 98(1): 77-87.

Cimadevilla, J. M., Y. Kaminsky, A. Fenton and J. Bures (2000). "Passive and active place avoidance as a tool of spatial memory research in rats." Journal of Neuroscience Methods 102(2): 155-164.

Clelland, C. D., M. Choi, C. Romberg, G. D. Clemenson, A. Fragniere, P. Tyers, et al. (2009). "A Functional Role for Adult Hippocampal Neurogenesis in Spatial Pattern Separation." Science 325(5937): 210-213.

Cobb, S. R., E. H. Buhl, K. Halasy, O. Paulsen and P. Somogyi (1995). "Synchronization of NeuronalActivity in Hippocampus by Individual GABAergic Interneurons." Nature 378(6552): 75-78.

Cohen, J., S. Pardy, H. Solway and H. Graham (2003). "Chunking versus foraging search patterns by rats in the hierarchically baited radial maze." Animal Cognition 6(2): 93-104.

Cohen, J. S., P. E. Mallet and K. Omalley (1993). Chunking By Proximal Arm Cues Facilitates Rats Performance In The Radial Maze. Meeting of the Psychonomic Soc, St Louis, Mo.

Collins, D. R., R. G. Pertwee and S. N. Davies (1995). "Prevention by the Cannabinoid Antagonist, SR141716A, of Cannabinoid-Mediated Blockade of Long-Term Potentiation in the Rat Hippocampal Slice." British Journal of Pharmacology 115(6): 869-870.

D'Hooge, R. and P. P. De Deyn (2001). "Applications of the Morris water maze in the study of learning and memory." Brain Research Reviews 36(1): 60-90.

Da Silva, G. E. and R. N. Takahashi (2002). "SR 141716A prevents Delta(9)-tetrahydrocannabinolinduced spatial learning deficit in a Morris-type water maze in mice." Progress in NeuroPsychopharmacology \& Biological Psychiatry 26(2): 321-325.

Dallal, N. L. and W. H. Meck (1990). "Hierarchical Structures - Chunking By Food Type Facilitates Spatial Memory." Journal of Experimental Psychology-Animal Behavior Processes 16(1): 69-84.

Davis, H. P. and L. R. Squire (1984). "Protein-Synthesis and Memory - A Review." Psychological Bulletin 96(3): 518-559.

De Lillo, C. (2004). "Imposing structure on a Corsi-type task: Evidence for hierarchical organisation based on spatial proximity in serial-spatial memory." Brain and Cognition 55(3): 415-426. 
Deng, W., J. B. Aimone and F. H. Gage (2010). "New neurons and new memories: how does adult hippocampal neurogenesis affect learning and memory?" Nature Reviews Neuroscience 11(5): 339-350.

Denise, A., R. Garcia, N. B. Doan, T. Imura, T. G. Bush and M. V. Sofroniew (2004). "GFAPexpressing progenitors are the principal source of constitutive neurogenesis in adult mouse forebrain." Nature Neuroscience 7(11): 1233-1241.

Devane, W. A., F. A. Dysarz, M. R. Johnson, L. S. Melvin and A. C. Howlett (1988). "Determination and Chatacterization of a Cannabinoid Receptor in Rat-Brain." Molecular Pharmacology 34(5): 605-613.

Devane, W. A., L. Hanus, A. Breuer, R. G. Pertwee, L. A. Stevenson, G. Griffin, et al. (1992). "Isolation and Structure if a Brain Constituent that Binds to the Cannabinoid Receptor." Science 258(5090): 1946-1949.

Dewey, W. L. (1986). "Cannabinoid Pharmacology." Pharmacological Reviews 38(2): 151-178.

Dotti, C. G., C. A. Sullivan and G. A. Banker (1988). "The establishment of polarity by hippocampal neurons in culture." Journal of Neuroscience 8(4): 1454-1468.

Drapeau, E. and D. N. Abrous (2008). "Stem cell review series: Role of neurogenesis in age-related memory disorders." Aging Cell 7(4): 569-589.

Dubreuil, D., C. Tixier, G. Dutrieux and J. M. Edeline (2003). "Does the radial arm maze necessarily test spatial memory?" Neurobiology of Learning and Memory 79(1): 109-117.

Dupret, D., A. Fabre, M. D. Dobrossy, A. Panatier, J. J. Rodriguez, S. Lamarque, et al. (2007). "Spatial learning depends on both the addition and removal of new hippocampal neurons." Plos Biology 5(8): 1683-1694.

Eichenbaum, H., C. Stewart and R. G. M. Morris (1990). "Hippocampal Representation in Place Learning." Journal of Neuroscience 10(11): 3531-3542.

Ennaceur, A. and J. Delacour (1988). "A New One-Trial Test for Neurobiological Studies of Memory in Rats .1. Behavioural Data." Behavioural Brain Research 31(1): 47-59.

Epp, J. R. and L. A. M. Galea (2009). "Hippocampus-dependent strategy choice predicts low levels of cell proliferation in the dentate gyrus." Neurobiology of Learning and Memory 91(4): 437-446.

Epp, J. R., M. D. Spritzer and L. A. M. Galea (2007). "Hippocampus-dependent learning promotes survival of new neurons in the dentate gyrus at a specific time during cell maturation." Neuroscience 149(2): 273-285.

Eriksson, P. S., E. Perfilieva, T. Bjork-Eriksson, A. M. Alborn, C. Nordborg, D. A. Peterson and F. H. Gage (1998). "Neurogenesis in the adult human hippocampus." Nature Medicine 4(11): 1313-1317.

Esser, L., C. R. Wang, M. Hosaka, C. S. Smagula, T. C. Sudhof and J. Deisenhofer (1998). "Synapsin I is structurally similar to ATP-utilizing enzymes." Embo Journal 17(4): 977-984.

Fan, N., H. W. Yang, J. Zhang and C. Chen (2010). "Reduced expression of glutamate receptors and phosphorylation of CREB are responsible for in vivo Delta 9-THC exposure-impaired hippocampal synaptic plasticity." Journal of Neurochemistry 112(3): 691-702.

Favaro, R., M. Valotta, A. L. M. Ferri, E. Latorre, J. Mariani, C. Giachino, et al. (2009). "Hippocampal development and neural stem cell maintenance require Sox2-dependent regulation of Sh." Nature Neuroscience 12(10): 1248-U62.

Feng, J., P. Chi, T. A. Blanpied, Y. M. Xu, A. M. Magarinos, A. Ferreira, et al. (2002). "Regulation of neurotransmitter release by synapsin III." Journal of Neuroscience 22(11): 4372-4380.

Fergusson, D. M. and J. M. Boden (2008). "Cannabis use and later life outcomes." Addiction 103(6): 969-976.

Ferraro, L., M. C. Tomasini, T. Cassano, B. W. Bebe, A. Siniscalchi, W. T. O'Connor, et al. (2001). "Cannabinoid receptor agonist WIN 55,212-2 inhibits rat cortical dialysate gammaaminobutyric acid levels." Journal of Neuroscience Research 66(2): 298-302. 
Ferreira, A., L. S. Chin, L. Li, L. M. Lanier, K. S. Kosik and P. Greengard (1998). "Distinct roles of synapsin I and synapsin II during neuronal development." Molecular Medicine 4(1): 22-28.

Ferreira, A., H. Q. Han, P. Greengard and K. S. Kosik (1995). "Suppression of synapsin-II inhibits the formation and maintenance of synapses in hippocampal culture." Proceedings of the National Academy of Sciences of the United States of America 92(20): 9225-9229.

Ferreira, A., H. T. Kao, J. Feng, M. Rapoport and P. Greengard (2000). "Synapsin III: Developmental expression, subcellular localization, and role in axon formation." Journal of Neuroscience 20(10): 3736-3744.

Ferri, A. L. M., M. Cavallaro, D. Braida, A. Di Cristofano, A. Canta, A. Vezzani, et al. (2004). "Sox2 deficiency causes neurodegeneration and impaired neurogenesis in the adult mouse brain." Development 131(15): 3805-3819.

Field, A. and S. Casswell (2000). Perspectives on Marijuana Policy in New Zealand: 1990 and 1998, University of Auckland.

Foster, T. C. (1999). "Involvement of hippocampal synaptic plasticity in age-related memory decline." Brain Research Reviews 30(3): 236-249.

Freund, T. F., I. Katona and D. Piomelli (2003). "Role of endogenous cannabinoids in synaptic signaling." Physiological Reviews 83(3): 1017-1066.

Fried, P. A., B. Watkinson and R. Gray (2005). "Neurocognitive consequences of marihuana - a comparison with pre-drug performance." Neurotoxicology and Teratology 27(2): 231-239.

Galiegue, S., S. Mary, J. Marchand, D. Dussossoy, D. Carriere, P. Carayon, et al. (1995). "Expression of Central and Peripheral Cannabinoid Receptors in Human Immune Tissues and Leukocyte Subpopulations." European Journal of Biochemistry 232(1): 54-61.

Gallagher, M. and P. C. Holland (1992). "Preserved Configural Learning and Spatial-Learning Impairment in Rats with Hippocmapal Damage." Hippocampus 2(1): 81-88.

Galve-Roperh, I., T. Aguado, D. Rueda, G. Velasco and M. Guzman (2006). "Endocannabinoids: A new family of lipid mediators involved in the regulation of neural cell development." Current Pharmaceutical Design 12(18): 2319-2325.

Ganeshina, O., R. W. Berry, R. S. Petralia, D. A. Nicholson and Y. Geinisman (2004). "Synapses with a segmented, completely partitioned postsynaptic density express more AMPA receptors than other axospinous synaptic junctions." Neuroscience 125(3): 615-623.

Gaoni, Y. and R. Mechoulam (1964). "Isolation Structure + Partial Synthesis of Active Constituent of Hashish." Journal of the American Chemical Society 86(8): 1646-\&.

Ge, S. Y., E. L. K. Goh, K. A. Sailor, Y. Kitabatake, G. L. Ming and H. J. Song (2006). "GABA regulates synaptic integration of newly generated neurons in the adult brain." Nature 439(7076): 589-593.

Ge, S. Y., C. H. Yang, K. S. Hsu, G. L. Ming and H. J. Song (2007). "A critical period for enhanced synaptic plasticity in newly generated neurons of the adult brain." Neuron 54(4): 559-566.

Geinisman, Y. (1993). "Perforated Axospinous Synapses with Multiple, Completely Partitioned Transmission Zones - Probable Structural Intermediates in Synaptic Plasticity." Hippocampus 3(4): 417-434.

Geinisman, Y. (2000). "Structural synaptic modifications associated with hippocampal LTP and behavioral learning." Cerebral Cortex 10(10): 952-962.

Geinisman, Y., L. Detoledomorrell and F. Morrell (1991). "Induction of Long-term Potentiation is Associated with an Increase in the Number of Axospinous Synapses with Segmented Postsynaptic Densities." Brain Research 566(1-2): 77-88.

Geinisman, Y., O. Ganeshina, R. Yoshida, R. W. Berry, J. F. Disterhoft and M. Gallagher (2004). "Aging, spatial learning, and total synapse number in the rat CA1 stratum radiatum." Neurobiology of Aging 25(3): 407-416.

Giancola, P. R., G. D. Shoal and A. C. Mezzich (2001). "Constructive thinking, executive functioning, antisocial behavior, and drug use involvement in adolescent females with a substance use disorder." Experimental and Clinical Psychopharmacology 9(2): 215-227. 
Ginsberg, S. D. and S. L. Che (2004). "Combined histochemical staining, RNA amplification, regional, and single cell cDNA analysis within the hippocampus." Laboratory Investigation 84(8): 952-962.

Glass, M., M. Dragunow and R. L. M. Faull (1997). "Cannabinoid receptors in the human brain: A detailed anatomical and quantitative autoradiographic study in the fetal, neonatal and adult human brain." Neuroscience 77(2): 299-318.

Gobet, F., P. C. R. Lane, S. Croker, P. C. H. Cheng, G. Jones, L. Oliver and J. M. Pine (2001). "Chunking mechanisms in human learning." Trends in Cognitive Sciences 5(6): 236-243.

Gomez-Pinilla, F., V. So and J. P. Kesslak (2001). "Spatial learning induces neurotrophin receptor and synapsin I in the hippocampus." Brain Research 904(1): 13-19.

Gould, E., A. Beylin, P. Tanapat, A. Reeves and T. J. Shors (1999). "Learning enhances adult neurogenesis in the hippocampal formation." Nature Neuroscience 2(3): 260-265.

Graham, V., J. Khudyakov, P. Ellis and L. Pevny (2003). "SOX2 functions to maintain neural progenitor identity." Neuron 39(5): 749-765.

Greengard, P., F. Valtorta, A. J. Czernik and F. Benfenati (1993). "Synaptic vesicle phosphoproteins and regulation of synaptic function." Science 259(5096): 780-785.

Greineisen, W. E. and H. Turner (2010). "Immunoactive effects of cannabinoids: Considerations for the therapeutic use of cannabinoid receptor agonists and antagonists." International Immunopharmacology 10(5): 547-555.

Grimsey, N. L., C. E. Goodfellow, E. L. Scotter, M. J. Dowie, M. Glass and E. S. Graham (2008). "Specific detection of CB1 receptors; cannabinoid CB1 receptor antibodies are not all created equal!" Journal of Neuroscience Methods 171(1): 78-86.

Hajos, N. and T. F. Freund (2002). "Pharmacological separation of cannabinoid sensitive receptors on hippocampal excitatory and inhibitory fibers." Neuropharmacology 43(4): 503-510.

Hajos, N., I. Katona, S. S. Naiem, K. Mackie, C. Ledent, I. Mody and T. F. Freund (2000). "Cannabinoids inhibit hippocampal GABAergic transmission and network oscillations." European Journal of Neuroscience 12(9): 3239-3249.

Hajos, N., C. Ledent and T. F. Freund (2001). "Novel cannabinoid-sensitive receptor mediates inhibition of glutamatergic synaptic transmission in the hippocampus." Neuroscience 106(1): 1-4.

Hall, W. and N. Solowij (1998). "Adverse effects of cannabis." Lancet 352(9140): 1611-1616.

Hampson, R. E. and S. A. Deadwyler (1999). "Cannabinoids, hippocampal function and memory." Life Sciences 65(6-7): 715-723.

Harvey, M. A., J. D. Sellman, R. J. Porter and C. M. Frampton (2007). "The relationship between non-acute adolescent cannabis use and cognition." Drug and Alcohol Review 26(3): 309319.

Hastings, N. B. and E. Gould (1999). "Rapid extension of axons into the CA3 region by adultgenerated granule cells." Journal of Comparative Neurology 413(1): 146-154.

Hayashi, Y., S. H. Shi, J. A. Esteban, A. Piccini, J. C. Poncer and R. Malinow (2000). "Driving AMPA receptors into synapses by LTP and CaMKII: Requirement for GluR1 and PDZ domain interaction." Science 287(5461): 2262-2267.

Heifets, B. D. and P. E. Castillo (2009). "Endocannabinoid Signaling and Long-Term Synaptic Plasticity." Annual Review of Physiology 71: 283-306.

Hellner, K., T. Walther, M. Schubert and D. Albrecht (2005). "Angiotensin-(1-7) enhances LTP in the hippocampus through the G-protein-coupled receptor Mas." Molecular and Cellular Neuroscience 29(3): 427-435.

Herkenham, M., A. B. Lynn, M. D. Little, M. R. Johnson, L. S. Melvin, B. R. Decosta and K. C. Rice (1990). "Cannabinoid Receptor Localization in Brain." Proceedings of the National Academy of Sciences of the United States of America 87(5): 1932-1936.

Heyser, C. J., R. E. Hampson and S. A. Deadwyler (1993). "Effects of Delta-9-tetrahydrocannabinol on Delayed Match to Sample Performance in Rats - Alterations in Short-term Memory 
Associated with Changes in Task Specific Firing of Hippocampal Cells." Journal of Pharmacology and Experimental Therapeutics 264(1): 294-307.

Hicks, A., S. Davis, J. Rodger, A. HelmeGuizon, S. Laroche and J. Mallet (1997). "Synapsin I and syntaxin 1B: Key elements in the control of neurotransmitter release are regulated by neuronal activation and long-term potentiation in vivo." Neuroscience 79(2): 329-340.

Hill, M. N., A. K. Titterness, A. C. Morrish, E. J. Carrier, T. T. Y. Lee, J. Gil-Mohapel, et al. (2010). "Endogenous Cannabinoid Signaling is Required for Voluntary Exercise-Induced Enhancement of Progenitor Cell Proliferation in the Hippocampus." Hippocampus 20(4): 513-523.

Hodges, H. (1996). "Maze procedures: The radial-arm and water maze compared." Cognitive Brain Research 3(3-4): 167-181.

Holson, R. R. and B. Pearce (1992). "Principles and Pitfalls in the Analysis of Prenatal Traatment Effects in Multiparous Species." Neurotoxicology and Teratology 14(3): 221-228.

Hosaka, M. and T. C. Sudhof (1998). "Synapsin III, a novel synapsin with an unusual regulation by Ca2+." Journal of Biological Chemistry 273(22): 13371-13374.

Howlett, A. C. (1995). "Pharmacology of Cannabinoid Receptors." Annual Review of Pharmacology and Toxicology 35: 607-634.

Howlett, A. C. and R. M. Fleming (1984). "Cannabinoid Inhibition of Adenylate Cyclase Pharmacology of the Response in Neuro-Blastoma Cell-Membranes." Molecular Pharmacology 26(3): 532-538.

Hsieh, C., S. Brown, C. Derleth and K. Mackie (1999). "Internalization and recycling of the CB1 cannabinoid receptor." Journal of Neurochemistry 73(2): 493-501.

Huestis, M. A. (2007). "Human cannabinoid pharmacokinetics." Chemistry \& Biodiversity 4(8): 1770-1804.

Inda, M. C., J. M. Delgado-Garcia and A. M. Carrion (2005). "Acquisition, consolidation, reconsolidation, and extinction of eyelid conditioning responses require de novo protein synthesis." Journal of Neuroscience 25(8): 2070-2080.

Iversen, L. (2003). "Cannabis and the brain." Brain 126: 1252-1270.

Jarvik, M. E. and R. Kopp (1967). "AN IMPROVED ONE-TRIAL PASSIVE AVOIDANCE LEARNING SITUATION." Psychological Reports 21(1): 221-\&.

Jessberger, S. and G. Kempermann (2003). "Adult-born hippocampal neurons mature into activitydependent responsiveness." European Journal of Neuroscience 18(10): 2707-2712.

Jiang, W., Y. Zhang, L. Xiao, J. Van Cleemput, S. P. Ji, G. Bai and X. Zhang (2005). "Cannabinoids promote embryonic and adult hippocampus neurogenesis and produce anxiolytic- and antidepressant-like effects." Journal of Clinical Investigation 115(11): 3104-3116.

Jin, K. L., L. Xie, S. H. Kim, S. Parmentier-Batteur, Y. J. Sun, X. O. Mao, et al. (2004). "Defective adult neurogenesis in CB1 cannabinoid receptor knockout mice." Molecular Pharmacology 66(2): 204-208.

Kaminski, N. E. (1993). Evedince for a Cannabinoid Receptor in Immunomodulation by Cannabinoid Compounds. Drugs of Abuse, Immunity, and Aids. H. Friedman, T. W. Klein and S. Specter. New York, Plenum Press Div Plenum Publishing Corp. 335: 115-120.

Kandel, E. R., J. H. Schwarts and T. M. Jessell (2000). Principles of Neural Science. United States of America, McGraw-Hill.

Kao, H. T., P. Li, H. M. Chao, S. Janoschka, K. Pham, J. Feng, et al. (2008). "Early involvement of synapsin III in neural progenitor cell development in the adult hippocampus." Journal of Comparative Neurology 507(6): 1860-1870.

Kao, H. T., B. Porton, A. J. Czernik, J. Feng, G. Yiu, M. Haring, et al. (1998). "A third member of the synapsin gene family." Proceedings of the National Academy of Sciences of the United States of America 95(8): 4667-4672. 
Katona, I., E. A. Rancz, L. Acsady, C. Ledent, K. Mackie, N. Hajos and T. F. Freund (2001).

"Distribution of CB1 cannabinoid receptors in the amygdala and their role in the control of GABAergic transmission." Journal of Neuroscience 21(23): 9506-9518.

Katona, I., B. Sperlagh, Z. Magloczky, E. Santha, A. Kofalvi, S. Czirjak, et al. (2000). "Gabaergic interneurons are the targets of cannabinoid actions in the human hippocampus." Neuroscience 100(4): 797-804.

Katona, I., B. Sperlagh, A. Sik, A. Kafalvi, E. S. Vizi, K. Mackie and T. F. Freund (1999). "Presynaptically located CB1 cannabinoid receptors regulate GABA release from axon terminals of specific hippocampal interneurons." Journal of Neuroscience 19(11): 45444558.

Kee, N., C. M. Teixeira, A. H. Wang and P. W. Frankland (2007). "Preferential incorporation of adult-generated granule cells into spatial memory networks in the dentate gyrus." Nature Neuroscience 10(3): 355-362.

Kennedy, M. B. (2000). "Signal-processing machines at the postsynaptic density." Science 290(5492): 750-754.

Kerr, D. S., L. R. M. Bevilaqua, J. S. Bonini, J. I. Rossato, C. A. Kohler, J. H. Medina, et al. (2005). "Angiotensin II blocks memory consolidation through an AT(2) receptor-dependent mechanism." Psychopharmacology 179(3): 529-535.

Kessels, R. P. C., E. H. F. de Haan, L. J. Kappelle and A. Postma (2001). "Varieties of human spatial memory: a meta-analysis on the effects of hippocampal lesions." Brain Research Reviews 35(3): 295-303.

Kim, D. and S. A. Thayer (2001). "Cannabinoids inhibit the formation of new synapses between hippocampal neurons in culture." Journal of Neuroscience 21(10): 5.

Kim, H. J., J. J. Waataja and S. A. Thayer (2008). "Cannabinoids inhibit network-driven synapse loss between hippocampal neurons in culture." Journal of Pharmacology and Experimental Therapeutics 325(3): 850-858.

Kirby, M. T., R. E. Hampson and S. A. Deadwyler (1995). "Cannabinoids Selectively Decrease Paired-Pulse Facilitation of Perforant Path Synaptic Potentials in the Dentate Gyrus InVitro." Brain Research 688(1-2): 114-120.

Klein, T. W., Y. Kawakami, C. Newton and H. Friedman (1991). "Marijuana Components Suppress Induction and Cytolytic Function of Murine Cytotoxic T-Cells In Vitro and In Vivo." Journal of Toxicology and Environmental Health 32(4): 465-477.

Knowlton, B. J. and M. S. Fanselow (1998). "The hippocampus, consolidation and on-line memory - Discussion point." Current Opinion in Neurobiology 8(2): 293-296.

Kochman, L. J., A. A. dos Santos, C. A. Fornal and B. L. Jacobs (2006). "Despite strong behavioral disruption, Delta(9)-tetrahydrocannabinol does not affect cell proliferation in the adult mouse dentate gyrus." Brain Research 1113: 86-93.

Kornau, H. C., P. H. Seeburg and M. B. Kennedy (1997). "Interaction of ion channels and receptors with PDZ domain proteins." Current Opinion in Neurobiology 7(3): 368-373.

Kreitzer, A. C., A. G. Carter and W. G. Regehr (2002). "Inhibition of interneuron firing extends the spread of endocannabinoid signaling in the cerebellum." Neuron 34(5): 787-796.

Kreitzer, A. C. and W. G. Regehr (2001). "Retrograde inhibition of presynaptic calcium influx by endogenous cannabinoids at excitatory synapses onto Purkinje cells." Neuron 29(3): 717727.

Kujtan, P. W., P. L. Carlen and B. M. Kapur (1983). "Delta-9-Tetrahydrocannabinol and Cannabidial - Dose-Dependent Effects on Evoked Potentials in the Hippocampal Slice." Canadian Journal of Physiology and Pharmacology 61(4): 420-426.

Le Foll, B., M. Wiggins and S. R. Goldberg (2006). "Nicotine pre-exposure does not potentiate the locomotor or rewarding effects of Delta-9-tetrahydrocannabinol in rats." Behavioural Pharmacology 17(2): 195-199. 
Leterrier, C., D. Bonnard, D. Carrel, J. Rossier and Z. Lenkei (2004). "Constitutive endocytic cycle of the CB1 cannabinoid receptor." Journal of Biological Chemistry 279(34): 36013-36021.

Leuner, B. and E. Gould (2010). "Structural Plasticity and Hippocampal Function." Annual Review of Psychology 61: 111-140.

Li, L., L. S. Chin, O. Shupliakov, L. Brodin, T. S. Sihra, O. Hvalby, et al. (1995). "Impairment of synapsic vesicle clustering and of synaptic transmission, and increased seizure propensity, in synapsin I-deficient mice." Proceedings of the National Academy of Sciences of the United States of America 92(20): 9235-9239.

Lichtman, A. H. (2000). "SR 141716A enhances spatial memory as assessed in a radial-arm maze task in rats." European Journal of Pharmacology 404(1-2): 175-179.

Lichtman, A. H., K. R. Dimen and B. R. Martin (1995). "Systemic Or Intrahippocampal Cannabinoid Administration Impairs Spatial Memory In Rats." Psychopharmacology 119(3): 282-290.

Lichtman, A. H., E. G. Hawkins, G. Griffin and B. F. Cravatt (2002). "Pharmacological activity of fatty acid amides is regulated, but not mediated, by fatty acid amide hydrolase in vivo." Journal of Pharmacology and Experimental Therapeutics 302(1): 73-79.

Lichtman, A. H. and B. R. Martin (1996). "Delta(9)-tetrahydrocannabinol impairs spatial memory through a cannabinoid receptor mechanism." Psychopharmacology 126(2): 125-131.

Lichtman, A. H., S. A. Varvel and B. R. Martin (2002). "Endocannabinoids in cognition and dependence." Prostaglandins Leukotrienes and Essential Fatty Acids 66(2-3): 269-285.

Liu, Y., T. Namba, J. Liu, R. Suzuki, S. Shioda and T. Seki (2010). "Glial Fibrillary Acidic ProteinExpression Neural Progenitors Give Rise to Immature Neurons via Early Intermediate Progenitors Expressing Both Glial Fibrillary Acidic Protein and Neuronal Markers in the Adult Hippocampus." Neuroscience 166(1): 241-251.

Livak, K. J. and T. D. Schmittgen (2001). "Analysis of relative gene expression data using real-time quantitative PCR and the 2(T)(-Delta Delta C) method." Methods 25(4): 402-408.

Llano, I., N. Leresche and A. Marty (1991). "Calcium Entry Increases the Sensitivity of Cerebellar Purkinje-Cells to Applied GABA and Decreases Inhibitory Synaptic Currents." Neuron 6(4): 565-574.

Long, L. H., R. L. Liu, F. Wang, J. Liu, Z. L. Hu, N. Xie, et al. (2009). "Age-Related Synaptic Changes in the CA1 Stratum Radiatum and Spatial Learning Impairment in Rats." Clinical and Experimental Pharmacology and Physiology 36(7): 675-681.

Lu, X., Y. Rong and M. Baudry (2000). "Calpain-mediated degradation of PSD-95 in developing and adult rat brain." Neuroscience Letters 286(2): 149-153.

Luebke, J. I., K. Dunlap and T. J. Turner (1993). "Multiple Calcium-Channel Types Control Glutamatergic Synaptic Transmission in the Hippocampus." Neuron 11(5): 895-902.

Luo, L., R. C. Salunga, H. Q. Guo, A. Bittner, K. C. Joy, J. E. Galindo, et al. (1999). "Gene expression profiles of laser-captured adjacent neuronal subtypes." Nature Medicine 5(1): 117-122.

Luscher, C., R. A. Nicoll, R. C. Malenka and D. Muller (2000). "Synaptic plasticity and dynamic modulation of the portsynaptic membrane." Nature Neuroscience 3(6): 545-550.

Lynskey, M. and W. Hall (2000). "The effects of adolescent cannabis use on educational attainment: a review." Addiction 95(11): 1621-1630.

Maccarrone, M., S. Rossi, M. Bari, V. De Chiara, F. Fezza, A. Musella, et al. (2008). "Anandamide inhibits metabolism and physiological actions of 2-arachidonoylglycerol in the striatum." Nature Neuroscience 11(2): 152-159.

Mackie, K. and B. Hille (1992). "Cannabinoids Inhibit N-type Calcium Channels in Neuroblastoma Glioma-Cells." Proceedings of the National Academy of Sciences of the United States of America 89(9): 3825-3829.

Mackie, K., Y. Lai, R. Westenbroek and R. Mitchell (1995). "Cannabinoids Activate am Inwardly Rectifying Potassium Conductance and Inhibit Q-Type Calcium Currents in ATT20 Cells Transfected with Rat-Brain Cannabinoid Receptor." Journal of Neuroscience 15(10): 65526561. 
Macuda, T. and W. A. Roberts (1995). "Further Evidence For Hierarchical Chunking In Rat Spatial Memory." Journal of Experimental Psychology-Animal Behavior Processes 21(1): 20-32.

Mallet, P. E. and R. J. Beninger (1996). "The endogenous cannabinoid receptor agonist anandamide impairs memory in rats." Behavioural Pharmacology 7(3): 276-284.

Mallet, P. E. and R. J. Beninger (1998). "The cannabinoid CB1 receptor antagonist SR141716A attenuates the memory impairment produced by Delta(9)-tetrahydrocannabinol or anandamide." Psychopharmacology 140(1): 11-19.

Mandel, R. J., F. H. Gage and L. J. Thal (1989). "Enhanced Detection of Nucleus Basalis Magnocellularis Lesion-Induced Spatial-Learning Deficit in Rats by Modification of Training Regimen." Behavioural Brain Research 31(3): 221-229.

Markakis, E. A. and F. H. Gage (1999). "Adult-generated neurons in the dentate gyrus send axonal projections to field CA(3) and are surrounded by synaptic vesicles." Journal of Comparative Neurology 406(4): 449-460.

Marsicano, G., S. Goodenough, K. Monory, H. Hermann, M. Eder, A. Cannich, et al. (2003). "CB1 cannabinoid receptors and on-demand defense against excitotoxicity." Science 302(5642): 84-88.

Martin, S. J., P. D. Grimwood and R. G. M. Morris (2000). "Synaptic plasticity and memory: An evaluation of the hypothesis." Annual Review of Neuroscience 23: 649-711.

Martini, L., M. Waldhoer, M. Pusch, V. Kharazia, J. Fong, J. H. Lee, et al. (2007). "Ligand-induced down-regulation of the cannabinoid 1 receptor is mediated by the G-protein-coupled receptor-associated sorting protein GASP1." Faseb Journal 21(3): 802-811.

Matsuda, L. A., S. J. Lolait, M. J. Brownstein, A. C. Young and T. I. Bonner (1990). "Structure of a Cannabinoid Receptor and Functional Expression of the Cloned cDNA." Nature 346(6284): 561-564.

Mechoulam, R., S. Benshabat, L. Hanus, M. Ligumsky, N. E. Kaminski, A. R. Schatz, et al. (1995). "Identification of an Endogenous 2-Monoglyceride, Present in Canine Gut, That Binds to Cannabinoid Receptors." Biochemical Pharmacology 50(1): 83-90.

Memberg, S. P. and A. K. Hall (1995). "Proliferation, Differentiation, and Survival of Rat Sensory Neuron Precursors in-vitro Require Specific Trophic Factors." Molecular and Cellular Neuroscience 6(4): 323-335.

Migaud, M., P. Charlesworth, M. Dempster, L. C. Webster, A. M. Watabe, M. Makhinson, et al. (1998). "Enhanced long-term potentiation and impaired learning in mice with mutant postsynaptic density-95 protein." Nature 396(6710): 433-439.

Miller, G. A. (1956). "The Magical Number 7, Plus Or Minus 2 - Some Limits On Our Capacity For Processing Information." Psychological Review 63(2): 81-97.

Mishima, K., N. Egashira, N. Hirosawa, M. Fujii, Y. Matsumoto, K. Iwasaki and M. Fujiwara (2001). "Characteristics of learning and memory impairment induced by Delta(9)tetrahydrocannabinol in rats." Japanese Journal of Pharmacology 87(4): 297-308.

Misner, D. L. and J. M. Sullivan (1999). "Mechanism of cannabinoid effects on long-term potentiation and depression in hippocampal CA1 neurons." Journal of Neuroscience 19(16): 6795-6805.

Molinaholgado, F., M. I. Gonzalez and M. L. Leret (1995). "Effect of Delta(9)-tetrahydrocannabinol on Short-term Memory in the Rat." Physiology \& Behavior 57(1): 177-179.

Monory, K., F. Massa, M. Egertova, M. Eder, H. Blaudzun, R. Westenbroek, et al. (2006). "The endocannabinoid system controls key epileptogenic circuits in the hippocampus." Neuron 51(4): 455-466.

Moore, N. L. T., A. L. R. Greenleaf, S. K. Acheson, W. A. Wilson, H. S. Swartzwelder and C. M. Kuhn (2010). "Role of Cannabinoid Receptor Type 1 Desensitization in Greater Tetrahydrocannabinol Impairment of Memory in Adolescent Rats." Journal of Pharmacology and Experimental Therapeutics 335(2): 294-301. 
Moriconi, A., I. Cerbara, M. Maccarrone and A. Topai (2010). "GPR55: Current Knowledge and Future Perspectives of a Purported "Type-3" Cannabinoid Receptor." Current Medicinal Chemistry 17(14): 1411-1429.

Moscovitch, M., R. S. Rosenbaum, A. Gilboa, D. R. Addis, R. Westmacott, C. Grady, et al. (2005). "Functional neuroanatomy of remote episodic, semantic and spatial memory: a unified account based on multiple trace theory." Journal of Anatomy 207(1): 35-66.

Moser, E., M. B. Moser and P. Andersen (1993). "Spatial-Learning Impairment Parallels the Magnitude of Dorsal Hippocampal-Lesions, but is Hardly Present Following Ventral Lesions." Journal of Neuroscience 13(9): 3916-3925.

Moser, E., M. B. Moser and P. Andersen (1993). "Spatial-Learning Impairment Parallels the Magnitude of Dorsal Hippocampal Lesions, but is Hardly Present Following Ventral Lesions." Journal of Neuroscience 13(9): 3916-3925.

Moser, M. B. (1999). "Making more synapses: a way to store information?" Cellular and Molecular Life Sciences 55(4): 593-600.

Mulder, J., T. Aguado, E. Keimpema, K. Barabas, C. J. B. Rosado, L. Nguyen, et al. (2008). "Endocannabinoid signaling controls pyramidal cell specification and long-range axon patterning." Proceedings of the National Academy of Sciences of the United States of America 105(25): 8760-8765.

Muller, D., N. Toni and P. A. Buchs (2000). "Spine changes associated with long-term potentiation." Hippocampus 10(5): 596-604.

Munro, S., K. L. Thomas and M. Abushaar (1993). "Molecular Characterization of a Peripheral Receptor for Cannabinoids." Nature 365(6441): 61-65.

Murphy, K. J., A. W. Oconnell and C. M. Regan (1996). "Repetitive and transient increases in hippocampal neural cell adhesion molecule polysialylation state following multitrial spatial training." Journal of Neurochemistry 67(3): 1268-1274.

Murphy, K. J. and C. M. Regan (1998). "Contributions of cell adhesion molecules to altered synaptic weightings during memory consolidation." Neurobiology of Learning and Memory 70(1-2): 73-81.

Murray, R. M., P. D. Morrison, C. Henquet and M. Di Forti (2007). "Science and society - Cannabis, the mind and society: the hash realities." Nature Reviews Neuroscience 8(11): 885-895.

Murray, R. M., P. D. Morrison, C. Henquet and M. D. Forti (2007). "Cannabis, the mind and society: the hash realities." Nat Rev Neurosci 8(11): 885-895.

Nacher, J., J. M. Blasco-Ibanez and B. S. McEwen (2002). "Non-granule PSA-NCAM immunoreactive neurons in the rat hippocampus." Brain Research 930(1-2): 1-11.

Nakamura, E. M., E. A. Dasilva, G. V. Concilio, D. A. Wilkinson and J. Masur (1991). "Reversible effects of acute and long-term administration of delta-9-tetrahydrocannabinol (THC) on memory in the rat." Drug and Alcohol Dependence 28(2): 167-175.

Navarrete, M. and A. Araque (2011). "Endocannabinoids Potentiate Synaptic Transmission through Stimulation of Astrocytes." Neuron 68(1): 113-126.

Navone, F., G. Digioia, R. Jahn, M. Browning, P. Greengard and P. Decamilli (1989). "Microvesicles of the neurohypophysis are biochemically related to small synaptic vesicles of presynaptic nerve-terminals." Journal of Cell Biology 109(6): 3425-3433.

Nayak, A. S., C. I. Moore and M. D. Browning (1996). "Ca2+/calmodulin-dependent protein kinase II phosphorylation of the presynaptic protein synapsin I is persistently increased during long-term potentiation." Proceedings of the National Academy of Sciences of the United States of America 93(26): 15451-15456.

Nelson, C., J. Bawa and S. Finger (1992). "Radial maze performance after hippocampal-lesions beneficial-effects of nimodipine." Restorative Neurology and Neuroscience 4(1): 33-40.

Nicholson, D. A., R. Yoshida, R. W. Berry, M. Gallagher and Y. Geinisman (2004). "Reduction in size of perforated postsynaptic densities in hippocampal axospinous synapses and age-related spatial learning impairments." Journal of Neuroscience 24(35): 7648-7653. 
Nilsson, O. G., M. L. Shapiro, F. H. Gage, D. S. Olton and A. Bjorklund (1987). "Spatial-Learning and Memory Following Fimbra-Fornix Transection and Grafting of Fetal Septal Neurons to the Hippocampus." Experimental Brain Research 67(1): 195-215.

Nowicky, A. V., T. J. Teyler and R. M. Vardaris (1987). "The Modulation of Long-Term Potentiation by Delta-9-Tetrahydrocannabinol in the Rat Hippocampus, In Vitro." Brain Research Bulletin 19(6): 663-672.

Nunzi, M. G., F. Milan, D. Guidolin and G. Toffano (1987). "Dendritic Spine Loss in Hippocampus of Aged Rats - Effect of Brain Phosphatidylserine Administration." Neurobiology of Aging 8(6): 501-510.

Nyffeler, M., B. K. Yee, J. Feldon and I. Knuesel (2010). "Abnormal differentiation of newborn granule cells in age-related working memory impairments." Neurobiology of Aging 31(11): 1956-1974.

Nyffeler, M., W. N. Zhang, J. Feldon and I. Knuesel (2007). "Differential expression of PSD proteins in age-related spatial learning impairments." Neurobiology of Aging 28(1): 143-155.

O'Shea, M., M. E. Singh, I. S. McGregor and P. E. Mallet (2004). "Chronic cannabinoid exposure produces lasting memory impairment and increased anxiety in adolescent but not adult rats." Journal of Psychopharmacology 18(4): 502-+.

Ohno-Shosaku, T., A. Tanimura, Y. Hashimotodani and M. Kano (2011). "Endocannabinoids and Retrograde Modulation of Synaptic Transmission." The Neuroscientist.

Ohno-Shosaku, T., H. Tsubokawa, I. Mizushima, N. Yoneda, A. Zimmer and M. Kano (2002). "Presynaptic cannabinoid sensitivity is a major determinant of depolarization-induced retrograde suppression at hippocampal synapses." Journal of Neuroscience 22(10): 38643872.

Olton, D. S., J. T. Becker and G. E. Handelmann (1979). "Hippocampus, Space, and Memory." Behavioral and Brain Sciences 2(3): 313-322.

Olton, D. S., C. Collison and M. A. Werz (1977). "Spatial Memory And Radial Arm Maze Performance Of Rats." Learning and Motivation 8(3): 289-314.

Olton, D. S. and B. C. Papas (1979). "Spatial Memory And Hippocampal Function." Neuropsychologia 17(6): 669-682.

Olton, D. S. and R. J. Samuelson (1976). "Remembrance Of Places Passed - Spatial Memory In Rats." Journal of Experimental Psychology-Animal Behavior Processes 2(2): 97-116.

Palmer, T. D., J. Takahashi and F. H. Gage (1997). "The adult rat hippocampus contains primordial neural stem cells." Molecular and Cellular Neuroscience 8(6): 389-404.

Paul, C. M., G. Magda and S. Abel (2009). "Spatial memory: Theoretical basis and comparative review on experimental methods in rodents." Behavioural Brain Research 203(2): 151164.

Pearce, J. M., A. D. L. Roberts and M. Good (1998). "Hippocampal lesions disrupt navigation based on cognitive maps but not heading vectors." Nature 396(6706): 75-77.

Peters, A., D. L. Rosene, M. B. Moss, T. L. Kemper, C. R. Abraham, J. Tigges and M. S. Albert (1996). "Neurobiological bases of age-related cognitive decline in the rhesus monkey." Journal of Neuropathology and Experimental Neurology 55(8): 861-874.

Peters, I. R., C. R. Helps, E. J. Hall and M. J. Day (2004). "Real-time RT-PCR: considerations for efficient and sensitive assay design." Journal of Immunological Methods 286(1-2): 203217.

Pieribone, V. A., B. Porton, B. Rendon, J. Feng, P. Greengard and H. T. Ka (2002). "Expression of synapsin III in nerve terminals and neurogenic regions of the adult brain." Journal of Comparative Neurology 454(2): 105-114.

Pieribone, V. A., O. Shupliakov, L. Brodin, S. Hilfikerrothenfluh, A. J. Czernik and P. Greengard (1995). "Distinct pools of synapsic vesicles in neurotransmitter release." Nature 375(6531): 493-497. 
Pisanu, A., E. Acquas, S. Fenu and G. Di Chiara (2006). "Modulation of Delta(9)-THC-induced increase of cortical and hippocampal acetylcholine release by mu opioid and D-1 dopamine receptors." Neuropharmacology 50(6): 661-670.

Pitler, T. A. and B. E. Alger (1992). "Postsynaptic Spike Firing Reduces Synaptic GABA(A) Responses in Hippocampal Pyramidal Cells." Journal of Neuroscience 12(10): 4122-4132.

Pope, H. G. and D. YurgelunTodd (1996). "The residual cognitive effects of heavy marijuana use in college students." Jama-Journal of the American Medical Association 275(7): 521-527.

Porton, B., R. M. Rodriguiz, L. E. Phillips, J. W. Gilbert, J. Feng, P. Greengard, et al. (2010). "Mice lacking synapsin III show abnormalities in explicit memory and conditioned fear." Genes Brain and Behavior 9(3): 257-268.

Pross, S., T. Klein, C. Newton and H. Friedman (1987). "DIFFERENTIAL-EFFECTS OF MARIJUANA COMPONENTS ON PROLIFERATION OF SPLEEN, LYMPH-NODE AND THYMUS-CELLS INVITRO." International Journal of Immunopharmacology 9(3): 363-370.

Puighermanal, E., G. Marsicano, A. Busquets-Garcia, B. Lutz, R. Maldonado and A. Ozaita (2009). "Cannabinoid modulation of hippocampal long-term memory is mediated by mTOR signaling." Nature Neuroscience 12(9): 1152-U18.

Quinn, H. R., I. Matsumoto, P. D. Callaghan, L. E. Long, J. C. Arnold, N. Gunasekaran, et al. (2008). "Adolescent rats find repeated Delta(9)-THC less aversive than adult rats but display greater residual cognitive deficits and changes in hippocampal protein expression following exposure." Neuropsychopharmacology 33(5): 1113-1126.

Ramirez-Amaya, V., D. F. Marrone, F. H. Gage, P. F. Worley and C. A. Barnes (2006). "Integration of new neurons into functional neural networks." Journal of Neuroscience 26(47): 1223712241.

Rapp, P. R. and M. Gallagher (1996). "Preserved neuron number in the hippocampus of aged rats with spatial learning deficits." Proceedings of the National Academy of Sciences of the United States of America 93(18): 9926-9930.

Rice, D. and S. Barone (2000). "Critical periods of vulnerability for the developing nervous system: Evidence from humans and animal models." Environmental Health Perspectives 108: 511533.

Richmond, M. A., B. K. Yee, B. Pouzet, L. Veenman, J. N. P. Rawlins, J. Feldon and D. M. Bannerman (1999). "Dissociating context and space within the hippocampus: Effects of complete, dorsal, and ventral excitotoxic hippocampal lesions on conditioned freezing and spatial learning." Behavioral Neuroscience 113(6): 1189-1203.

Ridgeway, D. (2006). "Strategic grouping in the spatial span memory task." Memory 14(8): 9901000.

Riedel, G., J. Micheau, A. G. M. Lam, E. V. Roloff, S. J. Martin, H. Bridge, et al. (1999). "Reversible neural inactivation reveals hippocampal participation in several memory processes." Nature Neuroscience 2(10): 898-905.

Robledo, P., F. Berrendero, A. Ozaita and R. Maldonado (2008). "Advances in the field of cannabinoid-opioid cross-talk." Addiction Biology 13(2): 213-224.

Rosahl, T. W., M. Geppert, D. Spillane, J. Herz, R. E. Hammer, R. C. Malenka and T. C. Sudhof (1993). "Short-term synaptic plasticity is altered in mice lacking synapsin-I." Cell 75(4): 661-670.

Rosahl, T. W., D. Spillane, M. Missler, J. Herz, D. K. Selig, J. R. Wolff, et al. (1995). "Essential functions of synapsin-I and synapsin-II in synaptic vesicle regulation." Nature 375(6531): 488-493.

Rubino, T., N. Realini, D. Braida, T. Alberio, V. Capurro, D. Virgano, et al. (2009). "The Depressive Phenotype Induced in Adult Female Rats by Adolescent Exposure to THC is Associated with Cognitive Impairment and Altered Neuroplasticity in the Prefrontal Cortex." Neurotoxicity Research 15(4): 291-302. 
Rubino, T., N. Realini, D. Braida, S. Guidi, V. Capurro, D. Vigano, et al. (2009). "Changes in Hippocampal Morphology and Neuroplasticity Induced by Adolescent THC Treatment are Associated With Cognitive Impairment in Adulthood." Hippocampus 19(8): 763-772.

Rubino, T., D. Vigano, N. Realini, C. Guidali, D. Braida, V. Capurro, et al. (2008). "Chronic Delta(9)tetrahydrocannabinol during adolescence provokes sex-dependent changes in the emotional profile in adult rats: Behavioral and biochemical correlates." Neuropsychopharmacology 33(11): 2760-2771.

Rueda, D., B. Navarro, A. Martinez-Serrano, M. Guzman and I. Galve-Roperh (2002). "The endocannabinoid anandamide inhibits neuronal progenitor cell differentiation through attenuation of the Rap1/B-Raf/ERK pathway." Journal of Biological Chemistry 277(48): 46645-46650.

Sairanen, M., G. Lucas, P. Ernfors, M. Castren and E. Castren (2005). "Brain-derived neurotrophic factor and antidepressant drugs have different but coordinated effects on neuronal turnover, proliferation, and survival in the adult dentate gyrus." Journal of Neuroscience 25(5): 1089-1094.

Sakai, K., K. Kitaguchi and O. Hikosaka (2003). "Chunking during human visuomotor sequence learning." Experimental Brain Research 152(2): 229-242.

Sanes, J. R. and J. W. Lichtman (1999). "Can molecules explain long-term potentiation?" Nature Neuroscience 2(7): 597-604.

Schneider, M. (2009). "Cannabis use in pregnancy and early life and its consequences: animal models." European Archives of Psychiatry and Clinical Neuroscience 259(7): 383-393.

Schneider, M. and M. Koch (2003). "Chronic pubertal, but not adult chronic cannabinoid treatment impairs sensorimotor gating, recognition memory, and the performance in a progressive ratio task in adult rats." Neuropsychopharmacology 28(10): 1760-1769.

Schoch, S., F. Deak, A. Konigstorfer, M. Mozhayeva, Y. Sara, T. C. Sudhof and E. T. Kavalali (2001). "SNARE function analyzed in synaptobrevin/VAMP knockout mice." Science 294(5544): 1117-1122.

Scholzen, T. and J. Gerdes (2000). "The Ki-67 protein: From the known and the unknown." Journal of Cellular Physiology 182(3): 311-322.

Scobie, K. N., B. J. Hall, S. A. Wilke, K. C. Klemenhagen, Y. Fujii-Kuriyama, A. Ghosh, et al. (2009). "Kruppel-Like Factor 9 Is Necessary for Late-Phase Neuronal Maturation in the Developing Dentate Gyrus and during Adult Hippocampal Neurogenesis." Journal of Neuroscience 29(31): 9875-9887.

Seri, B., J. Manuel, V. Garcia, L. Collado-Morente, B. S. McEwen and A. Alvarez-Buylla (2004). "Cell types, lineage, and architecture of the germinal zone in the adult dentate gyrus." Journal of Comparative Neurology 478(4): 359-378.

Shen, M. X., T. M. Piser, V. S. Seybold and S. A. Thayer (1996). "Cannabinoid receptor agonists inhibit glutamatergic synaptic transmission in rat hippocampal cultures." Journal of Neuroscience 16(14): 4322-4334.

Shi, L. H., F. Luo, D. J. Woodward and J. Y. Chang (2005). "Dose and behavioral context dependent inhibition of movement and basal ganglia neural activity by Delta(-9)tetrahydrocannabinol during spontaneous and treadmill locomotion tasks in rats." Synapse 55(1): 1-16.

Shors, T. J., G. Miesegaes, A. Beylin, M. R. Zhao, T. Rydel and E. Gould (2001). "Neurogenesis in the adult is involved in the formation of trace memories." Nature 410(6826): 372-376.

Simone, N. L., R. F. Bonner, J. W. Gillespie, M. R. Emmert-Buck and L. A. Liotta (1998). "Lasercapture microdissection: opening the microscopic frontier to molecular analysis." Trends in Genetics 14(7): 272-276.

Skibinska, A., M. Lech and M. Kossut (2001). "PSD95 protein level rises in murine somatosensory cortex after sensory training." Neuroreport 12(13): 2907-2910. 
Skinner, D. M., G. M. Martin, C. Harley, B. Kolb, A. Pridgar, A. Bechara and D. Vanderkooy (1994). "Acquisition of Conditional Discriminations in Hippocampal Lesioned and Decorticated Rats - Evidence for Learning that is Separate from both Simple Classical-Conditioning and Configural Learning." Behavioral Neuroscience 108(5): 911-926.

Slack, A., D. O'Dea, I. Sheerin, D. Narman, J. Wu and G. Nana (2008). New Zealand Drug Harm Index. Wellington, New Zealand, Business and Economic Research Limited.

Sloviter, R. S. and G. Nilaver (1987). "Immunocytochemical Localization of GABA-, CholecystokininLike, Vasoactive Intestinal Polypeptide-Like, and Somatostatin-Like Immunoreactivitu in the Area Dentata and Hippocampus of the Rat." Journal of Comparative Neurology 256(1): 42-60.

Small, S. A., W. Y. Tsai, R. DeLaPaz, R. Mayeux and Y. Stern (2002). "Imaging hippocampal function across the human life span: Is memory decline normal or not?" Annals of Neurology 51(3): 290-295.

Spear, L. P. (2000). "The adolescent brain and age-related behavioral manifestations." Neuroscience and Biobehavioral Reviews 24(4): 417-463.

Squire, L. R., C. E. L. Stark and R. E. Clark (2004). "The medial temporal lobe." Annual Review of Neuroscience 27: 279-306.

Stella, N., P. Schweitzer and D. Piomelli (1997). "A second endogenous cannabinoid that modulates long-term potentiation." Nature 388(6644): 773-778.

Stevens, C. F., S. Tonegawa and Y. Y. Wang (1994). "The Role of Calcium-calmodulin Kinase-II in 3 Forms of Synaptic Plasticity." Current Biology 4(8): 687-693.

Stiglick, A. and H. Kalant (1982). "Learning Impairment in the Radial Arm Maze Following Prolonger Cannabis Treatment in Rats." Psychopharmacology 77(2): 117-123.

Sudhof, T. C., A. J. Czernik, H. T. Kao, K. Takei, P. A. Johnston, A. Horiuchi, et al. (1989). "Synapsins - mosaics of shared and individual domains in a family of synaptic vesicle phosphoproteins." Science 245(4925): 1474-1480.

Suh, H., A. Consiglio, J. Ray, T. Sawai, K. A. D'Amour and F. H. Gage (2007). "In vivo fate analysis reveals the multipotent and self-renewal capacities of Sox2(+) neural stem cells in the adult hippocampus." Cell Stem Cell 1(5): 515-528.

Sullivan, J. M. (2000). "Cellular and molecular mechanisms underlying learning and memory impairments produced by cannabinoids." Learning \& Memory 7(3): 132-139.

Sun, W., A. Winseck, S. Vinsant, O. H. Park, H. Kim and R. W. Oppenheim (2004). "Programmed cell death of adult-generated hippocampal neurons is mediated by the proapoptotic gene Bax." Journal of Neuroscience 24(49): 11205-11213.

Sundram, S. (2006). "Cannabis and neurodevelopment: implications for psychiatric disorders." Human Psychopharmacology-Clinical and Experimental 21(4): 245-254.

Talpos, J. C., R. Dias, T. J. Bussey and L. M. Saksida (2008). "Hippocampal lesions in rats impair learning and memory for locations on a touch-sensitive computer screen: The "ASAT" task." Behavioural Brain Research 192(2): 216-225.

Tashiro, A., H. Makino and F. H. Gage (2007). "Experience-specific functional modification of the dentate gyrus through adult neurogenesis: A critical period during an immature stage." Journal of Neuroscience 27(12): 3252-3259.

Terrace, H. S. (1987). "Chunking By A Pigeon In A Serial-Learning Task." Nature 325(6100): 149151.

Terranova, J. P., J. C. Michaud, G. Lefur and P. Soubrie (1995). "Inhibition of Long-Term Potentiation in Rat Hippocampal Slices by Anandamide and WIN55212-2 - reversal by SR141716-A, a Selective Antagonist of CB1 Cannabinoid Receptors." NaunynSchmiedebergs Archives of Pharmacology 352(5): 576-579.

Trezza, V., V. Cuomo and L. Vanderschuren (2008). "Cannabis and the developing brain: Insights from behavior." European Journal of Pharmacology 585(2-3): 441-452. 
Tseng, A. H., J. W. Harding and R. M. Craft (2004). "Pharmacokinetic factors in sex differences in Delta(9)-tetrahydrocannabinol-induced behavioral effects in rats." Behavioural Brain Research 154(1): 77-83.

Tsou, K., S. Brown, M. C. Sanudo-Pena, K. Mackie and J. M. Walker (1998). "Immunohistochemical distribution of cannabinoid CB1 receptors in the rat central nervous system." Neuroscience 83(2): 393-411.

Twitchell, W., S. Brown and K. Mackie (1997). "Cannabinoids inhibit N- and P/Q-type calcium channels in cultured rat hippocampal neurons." Journal of Neurophysiology 78(1): 43-50.

Uysal, N., A. Dayi, S. Ozbal, F. Cetin, I. Aksu, G. Yalaz, et al. (2010). "Development of Spatial Memory During Adolescent Period in Rats." Journal of Neurological Sciences-Turkish 27(4): 407-413.

van Praag, H., A. F. Schinder, B. R. Christie, N. Toni, T. D. Palmer and F. H. Gage (2002). "Functional neurogenesis in the adult hippocampus." Nature 415(6875): 1030-1034.

VanGuilder, H. D., H. Yan, J. A. Farley, W. E. Sonntag and W. M. Freeman (2010). "Aging alters the expression of neurotransmission-regulating proteins in the hippocampal synaptoproteome." Journal of Neurochemistry 113(6): 1577-1588.

Varvel, S. A., R. J. Hamm, B. R. Martin and A. H. Lichtman (2001). "Differential effects of Delta(9)THC on spatial reference and working memory in mice." Psychopharmacology 157(2): 142-150.

Vaynman, S., Z. Ying and F. Gomez-Pinilla (2004). "Exercise induces BDNF and synapsin I to specific hippocampal subfields." Journal of Neuroscience Research 76(3): 356-362.

Veitch, N. C. (2004). "Horseradish peroxidase: a modern view of a classic enzyme." Phytochemistry 65(3): 249-259.

Venero, C., A. I. Herrero, K. Touyarot, K. Cambon, M. A. Lopez-Fernandez, V. Berezin, et al. (2006). "Hippocampal up-regulation of NCAM expression and polysialylation plays a key role on spatial memory." European Journal of Neuroscience 23(6): 1585-1595.

Vigano, D., T. Rubino and D. Parolaro (2005). "Molecular and cellular basis of cannabinoid and opioid interactions." Pharmacology Biochemistry and Behavior 81(2): 360-368.

von Bohlen und Halbach, O. (2005). "The renin-angiotensin system in the mammalian central nervous system." Current Protein \& Peptide Science 6(4): 355-371.

von Bohlen und Halbach, O. (2007). "Immunohistological markers for staging neurogenesis in adult hippocampus." Cell and Tissue Research 329(3): 409-420.

von Bohlen und Halbach, O., C. Zacher, P. Gass and K. Unsicker (2006). "Age-related alterations in hippocampal spines and deficiencies in spatial memory in mice." Journal of Neuroscience Research 83(4): 525-531.

Walch, A., K. Specht, J. Smida, M. Aubele, H. Zitzelsberger, H. Hofler and M. Werner (2001). "Tissue microdissection techniques in quantitative genome and gene expression analyses." Histochemistry and Cell Biology 115(4): 269-276.

Wang, X., D. Dow-Edwards, V. Anderson, H. Minkoff and Y. L. Hurd (2006). "Discrete opioid gene expression impairment in the human fetal brain associated with maternal marijuana use." Pharmacogenomics Journal 6(4): 255-264.

Ward, M. T., C. R. Stoelzel and E. J. Markus (1999). "Hippocampal dysfunction during aging II: Deficits on the radial-arm maze." Neurobiology of Aging 20(4): 373-380.

Watson, S., D. Chambers, C. Hobbs, P. Doherty and A. Graham (2008). "The endocannabinoid receptor, CB1, is required for normal axonal growth and fasciculation." Molecular and Cellular Neuroscience 38(1): 89-97.

Wheeler, D. B., A. Randall and R. W. Tsien (1994). "Roles of N-Type and Q-Type CA2+ Channels in Supporting Hippocampal Synaptic Transmission." Science 264(5155): 107-111.

Whishaw, I. Q., G. Mittleman, S. T. Bunch and S. B. Dunnett (1987). "Impairments in the Acquisition, Retention and Selection of Spatial Navigation Strategies After Medial Coudate-Putamen Lesions in rats." Behavioural Brain Research 24(2): 125-138. 
Williams, C. M. and T. C. Kirkham (2002). "Observational analysis of feeding induced by Delta(9)THC and anandamide." Physiology \& Behavior 76(2): 241-250.

Winner, B., Z. Kohl and F. H. Gage (2011). "Neurodegenerative disease and adult neurogenesis." European Journal of Neuroscience 33(6): 1139-1151.

Wise, L. E., P. A. Iredale and A. H. Lichtman (2008). "The cannabinoid CB1 receptor antagonist CE prolongs spatial memory duration in a rat delayed radial arm maze memory task." European Journal of Pharmacology 590(1-3): 246-249.

Wolf, S. A., A. Bick-Sander, K. Fabel, P. Leal-Galicia, S. Tauber, G. Ramirez-Rodriguez, et al. (2010). "Cannabinoid receptor CB1 mediates baseline and activity-induced survival of new neurons in adult hippocampal neurogenesis." Cell Communication and Signaling 8.

Wong, M. L. and J. F. Medrano (2005). "Real-time PCR for mRNA quantitation." Biotechniques 39(1): 75-85.

Yin, J. L., N. A. Shackel, A. Zekry, P. H. McGuinness, C. Richards, K. Van der Putten, et al. (2001). "Real-time reverse transcriptase-polymerase chain reaction (RT-PCR) for measurement of cytokine and growth factor mRNA expression with fluorogenic probes or SYBR Green I." Immunology and Cell Biology 79(3): 213-221.

Zehr, J. L., L. R. Nichols, K. M. Schulz and C. L. Sisk (2008). "Adolescent Development of Neuron Structure in Dentate Gyrus Granule Cells of Male Syrian Hamsters." Developmental Neurobiology 68(14): 1517-1526.

Zhao, C. M., W. Deng and F. H. Gage (2008). "Mechanisms and functional implications of adult neurogenesis." Cell 132(4): 645-660.

Zhou, D. and Z. H. Song (2001). "CB1 cannabinoid receptor-mediated neurite remodeling in mouse neuroblastoma N1E-115 cells." Journal of Neuroscience Research 65(4): 346-353.

Zurmohle, U., J. Herms, R. Schlingensiepen, W. Brysch and K. H. Schlingensiepen (1996). "Changes in the expression of synapsin I and II messenger RNA during postnatal rat brain development." Experimental Brain Research 108(3): 441-449.

Zygmunt, P. M., J. Petersson, D. A. Andersson, H. H. Chuang, M. Sorgard, V. Di Marzo, et al. (1999). "Vanilloid receptors on sensory nerves mediate the vasodilator action of anandamide." Nature 400(6743): 452-457. 